\title{
Analytical Approach to Eigen-Emittance in Storage Rings
}

\author{
Boaz Nash
}

\author{
Stanford Linear Accelerator Center \\ Stanford University \\ Stanford, CA 94309
}

SLAC-Report-820

Prepared for the Department of Energy
nder contract number DE-AC02-76SF00515

Printed in the United States of America. Available from the National Technical Information Service, U.S. Department of Commerce, 5285 Port Royal Road, Springfield, VA 22161. 
This document, and the material and data contained therein, was developed under sponsorship of the United States Government. Neither the United States nor the Department of Energy, nor the Leland Stanford Junior University, nor their employees, nor their respective contractors, subcontractors, or their employees, makes an warranty, express or implied, or assumes any liability of responsibility for accuracy, completeness or usefulness of any information, apparatus, product or process disclosed, or represents that its use will not infringe privately owned rights. Mention of any product, its manufacturer, or suppliers shall not, nor is it intended to, imply approval, disapproval, or fitness of any particular use. A royalty-free, nonexclusive right to use and disseminate same of whatsoever, is expressly reserved to the United States and the University. 


\title{
ANALYTICAL APPROACH TO EIGEN-EMITTANCE EVOLUTION IN STORAGE RINGS
}

\author{
A DISSERTATION \\ SUBMITTED TO THE DEPARTMENT OF PHYSICS \\ AND THE COMMITTEE ON GRADUATE STUDIES \\ OF STANFORD UNIVERSITY \\ IN PARTIAL FULFILLMENT OF THE REQUIREMENTS \\ FOR THE DEGREE OF \\ DOCTOR OF PHILOSOPHY
}

Boaz Nash

May 2006 
(C) Copyright by Boaz Nash 2006

All Rights Reserved 
I certify that I have read this dissertation and that, in my opinion, it is fully adequate in scope and quality as a dissertation for the degree of Doctor of Philosophy.

Alex Chao Principal Adviser

I certify that I have read this dissertation and that, in my opinion, it is fully adequate in scope and quality as a dissertation for the degree of Doctor of Philosophy.

Ron Ruth

I certify that I have read this dissertation and that, in my opinion, it is fully adequate in scope and quality as a dissertation for the degree of Doctor of Philosophy.

Bob Siemann

Approved for the University Committee on Graduate Studies. 


\section{Abstract}

This dissertation develops the subject of beam evolution in storage rings with nearly uncoupled symplectic linear dynamics. Linear coupling and dissipative/diffusive processes are treated perturbatively. The beam distribution is assumed Gaussian and a function of the invariants. The development requires two pieces: the global invariants and the local stochastic processes which change the emittances, or averages of the invariants. A map based perturbation theory is described, providing explicit expressions for the invariants near each linear resonance, where small perturbations can have a large effect. Emittance evolution is determined by the damping and diffusion coefficients. The discussion is divided into the cases of uniform and non-uniform stochasticity, synchrotron radiation an example of the former and intrabeam scattering the latter. For the uniform case, the beam dynamics is captured by a global diffusion coefficent and damping decrement for each eigen-invariant. Explicit expressions for these quantities near coupling resonances are given. In many cases, they are simply related to the uncoupled values. Near a sum resonance, it is found that one of the damping decrements becomes negative, indicating an anti-damping instability. The formalism is applied to a number of examples, including synchrobetatron coupling caused by a crab cavity, a case of current interest where there is concern about operation near half integer $\nu_{x}$. In the non-uniform case, the moment evolution is computed directly, which is illustrated through the example of intrabeam scattering. Our approach to intrabeam scattering damping and diffusion has the advantage of not requiring a loosely-defined Coulomb Logarithm. It is found that in some situations there is a small difference between our results and the standard approaches such as Bjorken-Mtingwa, which is illustrated by comparison of the two approaches and with 
a measurement of $\mathrm{Au}$ evolution in RHIC. Finally, in combining IBS with the global invariants some general statements about IBS equilibrium can be made. Specifically, it is emphasized that no such equilibrium is possible in a non-smooth lattice, even below transition. Near enough to a synchrobetatron coupling resonance, it is found that even for a smooth ring, no IBS equilibrium occurs. 


\section{Acknowledgments}

The field of accelerator physics is very broad. What has been a continuous source of surprise and interest for me is the depth of the subject. I wish to thank Ron Ruth for originally introducing me to this subject and giving me a glimmer of understanding of its riches.

It has been a true privilege to have Alex Chao as my thesis advisor. His continual confidence in both me and in accelerator/beam physics has pushed me along the difficult path to this dissertation. He has applied just enough pressure to keep me from getting bogged down in overly abstract speculation, as well as quickly realizing when a promising path should not be dropped until it has born some fruit. His great knowledge of the field and ability to get to the core issue of any complex problem has consistently taught and inspired me.

I also wish to express my great appreciation to Juhao $\mathrm{Wu}$, who has worked with me steadily for all these years. Research can be a lonely, solitary activity, and his discussions, as well as his hard work and insights have helped me and this work in innumerable ways. Next, I thank Ben Freivogel who followed the progress of this work, providing help at key moments of confusion, even though his own specialization in theoretical physics is far removed from the motion of charged particles in electromagnetic fields.

Thanks go to my thesis committee: Bob Siemann, Yunhai Cai, Alex Chao, Ron Ruth and Amir Dembo for their suggestions and comments which have improved this dissertation. Finally, it is with pleasure that I acknowledge Karl Bane for collaboration in the work on intrabeam scattering and Wolfram Fischer for helpful discussions and providing me with RHIC data and parameters. 
This work is supported by the U.S. Department of Energy under Contract No. DE-AC0-76SF00515. 


\section{Contents}

Abstract iv

Acknowledgments vi

1 Introduction $\quad 1$

2 General Overview 12

2.1 Linear Dynamics and Invariants . . . . . . . . . . . . . . . . 14

2.2 Unperturbed Case . . . . . . . . . . . . . . . . . . . . . . . . . . 19

2.3 Smooth Storage Ring . . . . . . . . . . . . . . . . . . . . . . . . . . . . . . . . . . .

2.4 Non-symplectic process . . . . . . . . . . . . . . . . . . 25

2.5 Reversible/Irreversible Dynamics . . . . . . . . . . . . . . . 29

3 Perturbation Theory Near Linear Resonances 31

3.1 Perturbation to Eigenvalues and Eigenvectors . . . . . . . . . . . 34

3.2 Cases of Resonances . . . . . . . . . . . . . . . . . . . . . 41

3.2.1 Integer/Half Integer Resonance . . . . . . . . . . . . . . . 41

3.2 .2 Sum Resonance . . . . . . . . . . . . . . . . . . . . . . . . . 44

3.2.3 Difference Resonance . . . . . . . . . . . . . . . . . . 45

3.2.4 Special Case of an Integer/Half-Integer Resonance Caused by Coupling . . . . . . . . . . . . . . 46

3.3 Evolution of Eigenvectors Around Ring . . . . . . . . . . . . . . 50

3.4 Invariants . . . . . . . . . . . . . . . . . . . . 52

3.4.1 Sum/Difference resonance . . . . . . . . . . . . 53 
3.4 .2 Integer/Half-integer Resonance . . . . . . . . . . 54

3.5 Sum Rules . . . . . . . . . . . . . . . . . . . . . . . . 55

3.6 x-y coupling: skew quadrupole . . . . . . . . . . . . . . 57

3.6.1 Numerical Instability Plot . . . . . . . . . . . . . . . . . . 58

3.7 x-z Coupling: Dispersion at an RF Cavity . . . . . . . . . . . . 58

3.7.1 Sum/Difference Resonance . . . . . . . . . . . . . . . . . . . 60

3.7 .2 Integer/Half-integer Resonance . . . . . . . . . . . . . . 61

3.8 Crab Cavity . . . . . . . . . . . . . . . . . . . . . . . . . . 63

3.8.1 Sum/Difference Resonance . . . . . . . . . . . . . . . 64

3.8.2 Integer/Half-integer Resonance . . . . . . . . . . . . 66

4 Constant Damping/Diffusion $\quad 69$

4.1 Sum Rules . . . . . . . . . . . . . . . . . . . . . . . . 72

4.2 Example: Synchrotron Radiation _. . . . . . . . . . . . . . . 74

5 Coupling and Constant Damping/Diffusion Combined 81

5.1 Diffusion Coefficients . . . . . . . . . . . . . . . . . . . . . 81

5.1.1 Sum/Difference Resonance . . . . . . . . . . . . . . . . . . . 82

5.1 .2 Integer/Half-integer Resonance . . . . . . . . . . . . 83

5.2 Damping Coefficients . . . . . . . . . . . . . . . . . . 84

5.2.1 Sum/Difference Resonance . . . . . . . . . . . . . . . . 84

5.2.2 Integer/Half-integer Resonances . . . . . . . . . . . . . 86

5.3 Equilibrium Eigen-Emittances . . . . . . . . . . . . . . . 86

5.4 Sigma Matrices . . . . . . . . . . . . . . . . . . . . . . . 88

5.4.1 Beam Profile Near Difference Resonance . . . . . . . . . . . . 91

5.4.2 Projected Emittance Sum Rules . . . . . . . . . . . . . . . . . 92

5.5 Applications . . . . . . . . . . . . . . . . . . . . . . 94

5.6 Global Quantities Near Sum/Difference Resonances . . . . . . . . . . 94

5.7 Anti-damping Instability . . . . . . . . . . . . . . . . . . . . 94

5.8 Dispersion at Crab Cavity . . . . . . . . . . . . . . . . . . . . 102

5.9 Numerical Results . . . . . . . . . . . . . . . . . . . . . . . . . . 102

5.10 Instabilities Near Half-Integer $\nu_{x} \quad \ldots \ldots$. . . . . . . . . . . . 105 
5.11 Instabilities Near Integer $\nu_{x} \ldots \ldots$. . . . . . . . . . . 107

5.12 Single Versus Multiple Resonances . . . . . . . . . . . . . . . . . . . . 108

5.13 Discussion . . . . . . . . . . . . . . . . . . . . . 109

6 Non-Constant Damping/Diffusion 111

6.1 IBS Dissipation/Diffusion . . . . . . . . . . . . . . . . . . . . 112

6.2 Uncoupled Case . . . . . . . . . . . . . . . . . . 126

6.3 Equilibrium distributions . . . . . . . . . . . . . . . . 133

6.4 Overview of IBS Literature . . . . . . . . . . . . . . . . . . 135

6.5 Beam-Beam Diffusion . . . . . . . . . . . . . . . . . . . 136

7 Coupled IBS and Combination with SR 139

7.1 Coupled Matrices . . . . . . . . . . . . . . . . . . 139

7.2 Coupled Emittance Evolution and Combination with Synchrotron Radiation . . . . . . . . . . . . . . . . . . . 140

7.3 Coupled Piwinski Invariant . . . . . . . . . . . . . . . . . . . . 141

8 Conclusions and Future Work 143

$\begin{array}{ll}\text { A Further Invariant Calculations } & 148\end{array}$

B General Perturbation Theory 152

$\begin{array}{lr}\text { C Relationship between } \xi_{+} \text {and } \xi_{-} & 157\end{array}$

D Coupling Integer/Half Integer Resonances $\quad 159$

$\begin{array}{lr}\text { E IBS Calculations } & 164\end{array}$

$\begin{array}{lr}\text { Bibliography } & 178\end{array}$ 


\section{List of Tables}

3.1 Linear resonances of a synchrobetatron coupled storage ring. For each of the linear resonances, we give general expressions for the quantities $\Delta \mu, \xi, \phi$, and $\bar{\mu}$. The quantities $r_{j k}$ here are $r_{j k \xi}$, i.e. the part of $r_{j k}$ coming from the coupling perturbation $P_{\xi}$. We have left off the subscript $\xi$ for convenience. The matrix elements $r_{j k \mu}$ coming from the difference from resonance are included explicitly in the $\Delta \mu$ and involved $\mu_{x}$ and $\mu_{z}$. The resonances with the abbreviation "cp." refer to the full 2 nd order calculation for the coupling-induced integer and half integer resonances (see Appendix D). . . . . . . . . . . . . . . 47

3.2 Resonances for dispersive RF cavity . . . . . . . . . . . . . . . . 63

3.3 Resonances for Crab Cavity . . . . . . . . . . . . . . . . . . . 68

5.1 This table contains the diffusion coefficients near each of the linear resonances. The $d$ column gives the local diffusion coefficient for modes 1 and 2. The $d_{c}$ column gives the formula for the extra term $d_{c}$ contained in the corresponding local diffusion coefficient. Finally, the $\bar{d}$ column contains the global diffusion coefficient which is given by integrating the local quantity. The text discusses the approximations used for these expressions. The angle $\theta$ is given by $\tan ^{-1}(\xi / \Delta \mu)$ for the difference resonance and $\tanh ^{-1}(\xi / \Delta \mu)$ for the other resonances where $\xi$ and $\Delta \mu$ are given in table 3.1. The phase $\phi$ for the integer $z$ resonance is also given in table $3.1 . \ldots \ldots \ldots \ldots$ 
5.2 This table contains the damping coefficients near each of the linear resonances. The $b$ column gives the local damping coefficient for modes 1 and 2. The $b_{c}$ column tells where to find the formula for the extra term $b_{c}$ contained in the corresponding local damping coefficient. Finally, the $\chi$ column contains the global damping decrement which is given by integrating the local quantity. The approximations used for these expressions are discussed in the text. The angle $\theta$ is given by $\tan ^{-1}(\xi / \Delta \mu)$ for the difference resonance and $\tanh ^{-1}(\xi / \Delta \mu)$ for the other resonances where $\xi$ and $\Delta \mu$ are given in table $3.1 . . . . .$.

5.3 Parameters used in our numerical examples based on the PEP-II Low Energy Storage Ring[59] . . . . . . . . . . . . . . . . . . . . . . 95

6.1 ATF, RHIC Parameters . . . . . . . . . . . . . . . . . . . 129 


\section{List of Figures}

3.1 Instability plot for coupling due to skew quad with $\xi_{c}=0.25$. The horizontal axis is $\nu_{x}$ and the vertical is $\nu_{y} \ldots \ldots \ldots$

5.1 Here we plot the perturbed and unperturbed tunes with the addition of coupling as the unperturbed tune split is varied. For the case of transverse coupling, the tune split can be varied by changing the strength of a quadrupole magnet. The ellipses show contours of the beam distribution in $x_{\beta}-z_{\beta}$ space. The outer two ellipses represent the beam relatively far from the coupling resonance. The second and fourth ellipses represent the beam at approximately $\theta=45^{\circ}$, while the center round beam is exactly on the coupling resonance at $\theta=90^{\circ}$. The unperturbed phase advances $\mu_{x}$ and $\mu_{z}$ are purple and red respectively. With coupling, $\mu_{x}$ and $\mu_{z}$ become $\mu_{1}$ and $\mu_{2}$ which are represented by the green and blue curves respectively. The global coupling parameter is $\xi=0.003$ which can be read from the difference between $\mu_{1}$ and $\mu_{2}$ exactly on resonance. Note that the identity of $\mu_{1}$ and $\mu_{2}$ switch discontinuously with respect to the continuous curves in the upper and lower regions of the diagram. We have assumed that we are at a position in the ring where $\phi=0$. For different values of $\phi$, the second and fourth of the beam distributions would be rotated at different angles. 
5.2 In this figure, we plot global damping, diffusion and equilibrium invariant values for coupling due to a dispersion of 1 meter at an RF cavity near the sum/difference resonances. The upper two rows are the sum resonance, and lower two are the difference resonance. Parameters are otherwise drawn from Table 5.3 based on the PEP-II LER. The quantities are plotted as a function of the betatron tune $\nu_{x}$ and the synchrotron tune $\nu_{s}$ which is positive and equal to $-\nu_{z}$, thus giving an inversion of sum and difference resonances. $\chi_{1}$ and $\chi_{2}$ are global damping decrements expressed in Table 5.2. $\bar{d}_{1}$ and $\bar{d}_{2}$ are global diffusion coefficients expressed in Table 5.1, and $\left\langle g_{1}\right\rangle_{\text {eq }}$ and $\left\langle g_{2}\right\rangle_{\text {eq }}$ are one half the ratio of these quantities as given by Eq.(4.10). All quantities have been divided by their uncoupled values so that the blue region with the value of 1 represents no effect from coupling. The region of instability due to the Hamiltonian dynamics is black. There is also an extremely small region of anti-damping instability outside the symplectic instability region for the sum resonance where the damping decrement $\chi_{1}$ is negative. This region is indicated by white. . . . . . . . . . . . . . . . .

5.3 In this figure, we plot global damping, diffusion and equilibrium invariant values for coupling due to a crab cavity with $\xi_{c}=0.003$ near the sum and difference resonances. The dispersion at the crab cavity is set to 0 in this example. Because the coupling strength $\xi$ is inversely proportional to $\sqrt{\nu_{s}}$, the instability broadens for smaller $\nu_{s} . \chi_{1}, \chi_{2}, \bar{d}_{1}, \bar{d}_{2},\left\langle g_{1}\right\rangle$ and $\left\langle g_{2}\right\rangle$ are the same as in Fig.5.2. As in Fig. 5.2 , the small region of anti-damping for $\chi_{1}$ near the sum resonance is colored white. 97

5.4 Projected emittances near the sum and difference resonances due to dispersion at the RF cavity. Paramaters are the same as those in Figs.5.2. The upper two plots are for the sum resonance with $\epsilon_{x, \mathrm{pr}}^{+}$and $\epsilon_{z, \text { pr }}^{+}$defined in Eqs. (5.28) and (5.29). The lower two plots are for the difference resonance with $\epsilon_{x, \text { pr }}^{-}$and $\epsilon_{z, \text { pr }}^{-}$defined in Eqs.(5.31) and (5.32). 98

5.5 Projected emittances near the sum and difference resonances due to a crab cavity. The four plots have the same meanings as in Fig.5.4. Parameters are the same as those in Figs.5.3. . . . . . . . . . . . . 
5.63 -D plots of projected emittances near the sum and difference resonances due to a dispersive RF cavity. The four plots have the same meanings as in Fig.5.4. Parameters are the same as those in Figs.5.2. 100

5.7 3-D plots of projected emittances near the sum and difference resonances due to a crab cavity. The four plots have the same meanings as in Fig.5.4. Parameters are the same as those in Figs.5.3 . . . . . . . 101

5.8 Damping decrement near sum resonance for coupling due to a dispersive RF cavity (upper plots) and a crab cavity (lower plots). The black region represents an instability in the Hamiltonian dynamics, whereas the white area represents a negative value of $\chi_{1}$ and hence an antidamping instability. We vary the damping decrement $\mathcal{D}$. In the two left-most plots it is 0.25 . For the center plots it is 0.5 , and the right plots have 0.75. The plots of $\chi_{1}$ near the sum resonance in Figs.5.2 and 5.3 plot the same quantities, except that in those cases, $\mathcal{D} \approx 0$ as discussed in the text. Increasing $\mathcal{D}$ increases the ratio of $\chi_{z}$ and $\chi_{x}$ which widens the region where an anti-damping instability occurs. . . 103 
5.9 These six plots relate to crab cavity coupling. The upper two plots are instability diagrams. The eigenvalues $\lambda_{j}$, have been computed numerically for each value of the tunes and if for each $j,\left|\lambda_{j}\right|=1$, it is stable and colored white, whereas $\lambda_{j} \neq 1$ is unstable and colored black. The left plot has no dispersion at the crab cavity, while the right plot has a dispersion of 3 meters. The middle two plots show a magnified view of the upper two plots near $\nu_{x}=1 / 2$. Note that the $\nu_{x}$ half integer resonance caused by dispersion is only visible upon magnification. The integer $\nu_{x}$ resonance also appears with dispersion as can be seen from the thin black line near $\nu_{x}=0$ in the upper right plot. The bottom two plots give the horizontal and longitudinal equilibrium invariants, $\left\langle g_{1}\right\rangle_{\text {eq }}$ and $\left\langle g_{2}\right\rangle_{\text {eq }}$ divided by their uncoupled values, defined in Eq.(4.10). Because $\chi_{1,2}=\chi_{x, z}$ for integer and half-integer resonances, this is also given by the ratios $\bar{d}_{1} / \bar{d}_{x}$ and $\bar{d}_{2} / \bar{d}_{z}$ where $\bar{d}_{1}$ and $\bar{d}_{2}$ are defined in Table 5.1. Explicit expressions for the emittance growth for transverse and longitudinal are given in Eqs. (5.49) and (5.52). We have set the dispersion at the crab cavity to zero here. The bottom left is plotted as a function of $\nu_{x}$ and $\nu_{s}$, while the bottom right is plotted as a function of $\nu_{x}$ and dispersion at the crab cavity in units of meters. The $\nu_{x}$ half-integer instability does not depend on $\nu_{s}$. . . . . . . . .

5.10 Comparison of eigenvalue formula to direct numerical calculation. Here, $\nu_{x}=0.51$, and we plot the absolute value of $\mu_{2}$, which becomes imaginary (unstable) due to the integer $\mu_{s}=0$ resonance. We have set the dispersion at the crab cavity to be $\eta=0.1 \mathrm{~m}$. The red is the numerical result, while the blue is Eq.(3.160).

6.1 Coulomb Logs for ATF . . . . . . . . . . . . . . . .

6.2 Comparison of B-M to our formulae in reproducing RHIC AU bunch length data $[72,73]$ The vertical axis is RMS bunch length measured from a wall current monitor, and time is measured in seconds. The blue points represent the bunch length data. The red points give bunch length evolution using our equations which we computed using Eq. (6.70) and the purple dots represent the results of Bjorken-Mtingwa, Eq. (6.74) 
6.3 Equilibrium value of $\tilde{C}$ as a function of $\tilde{A}$ for several different values of $L_{c}=\frac{1}{2} \log A$ ("Coulomb Log"). $L_{c}=5$ is yellow, $L_{c}=10$ is green, $L_{c}=15$ is blue, and $L_{c}=20$ is purple. . . . . . . . . . . . . 134

6.4 Same as previous figure with larger range of $\tilde{A}$. . . . . . . . . . . . . 134

7.1 Evolution of Coupled Emittances for ATF . . . . . . . . . . . . . 141 


\section{Chapter 1}

\section{Introduction}

A storage ring is a device in which a high energy beam of charged particles is confined in the vicinity of a closed orbit. Storage rings can be used for particle acceleration, colliders for nuclear and particle physics experiments, damping rings and light sources. Intensive research is ongoing for all of these applications with energy being pushed higher, beam intensity increased and control and measurement of other beam properties pushed to the limits of current technology and understanding. The motivation for the present dissertation arose out of several issues that researchers confront as these various limits are pushed.

The first problem is that of intrabeam scattering (IBS). Particles in the beam interact via the Coulomb interaction. This causes particles to slowly move off the orbits prescribed by the external magnetic fields. Because of the essential randomness of the detailed particle distribution this causes beam evolution in a thermal equilibration like process. At a single particle level, one can think of IBS as causing diffusion and damping (so-called "dynamical friction"). The effects of IBS get stronger with increased particle density which results from either increased beam current or reduced beam sizes. However, it is strongly energy-dependent, making it most relevant for machines with low and intermediate energies. For example, in the ALS, a third generation light source, IBS is a strong effect at $0.7 \mathrm{GeV}$, but very weak at $1.9 \mathrm{GeV}$ [1].

Intrabeam scattering theory has a long history, passing through such disparate 
subjects as galactic dynamics (see e.g. $[2,3,4]$ ) and plasma physics (see e.g. [10, 11]) before being adapted to charged particle beams. The connection of course, is that both the gravitational and Coulomb interactions are $1 / r^{2}$ forces; attraction or repulsion ends up being relatively unimportant here. The two main adaptations of this theory to accelerator physics are those of A. Piwinski [12] and Bjorken and Mtingwa (BM) [13].

There are two reasons for revisiting IBS theory which we will focus on here. The first is the issue of the Coulomb Logarithm. This is a parameter that shows up in the standard IBS analysis and is usually expressed as the logarithm of the ratio of a maximum to minimum impact parameter for the scattering process. The minimum distance cut-off is relatively uncontroversial: a variety of different arguments all lead to a distance of the order of the distance of minimum approach of the two particles. The large distance cut-off $b_{\max }$ has been more controversial, however. The question is one of locality. If only nearby particles need to be considered, then one takes $b_{\max }$ to be of the order of the inter-particle spacing. If no such locality condition can be assumed, then one must include scatters up to some possibly much larger distance scale. In plasma physics, for a uniform density plasma, an argument based on charge shielding gives the Debye length for this length scale. In a non-neutral situation of either stars interacting gravitationally, or a charged particle beam, however, such a shielding cut-off does not obviously apply ${ }^{1}$. For a view into the controversy, see e.g. $[15,16,17]$.

By the time diffusion and damping due to the Coulomb interaction was adapted to accelerator physics, the controversy was mostly seen as resolved with the "system size" determining $b_{\max }{ }^{2}$. Let us suppose that in some sense this is the correct answer. Charged particle beams give this issue a further twist however. In modern light sources or damping rings the vertical beam size can be much smaller than the horizontal which in turn is much smaller than the bunch length. Which of these three sizes should be used? Typically, the smallest of the three sizes is taken, but one may

\footnotetext{
${ }^{1}$ For the gravitational case, some have suggested using the distance associated with the Jeans instability[14].

${ }^{2}$ Note, however, that in Piwinski's 1974 original paper, he takes the interparticle spacing for determining $b_{\max }$. His later work suggests using the beam size.
} 
wonder whether this is justified. In this dissertation, we describe an approach to IBS in which no large distance cut-off is required. The scattering integrals are cut off naturally by the actual beam distribution ${ }^{3}$. This approach was first described in [18]. Here we give a more complete treatment.

The second issue regarding IBS theory relates to coupling. The original formulations of Piwinski and BM didn't include a coupled beam distribution. Piwinski made some progress on this topic in [19] as did V. Lebedev [20] more recently in unpublished work. General coupling is also combined with IBS in the computer program $\mathrm{SAD}$, with an approach as described in [21]. All of these approaches involve general coupling and are mainly amenable to numerical computation. One may wish for an intermediate approach that includes coupling but still allows for analytical understanding. Such an approach by the present author and collaborators which included transverse coupling and was applied to understanding of IBS data in the KEK ATF was described in [18]. Elaborating on the approach described in this work amounts to a substantial portion of the contents of this dissertation.

The topic of linear coupling is still an active research subject (see e.g. [24, 25, 26, $27,28]$ ), despite the fact that general analyses were initiated quite some time ago[22, 23]. Because of its importance in accelerator operations, improved understanding of parametrization, measurement, and correction continues development.

These references relate mainly to transverse (betatron) coupling. There is also the issue of transverse-longitudinal (synchro-betatron) coupling [44]. A recent reason for wanting to understand this issue involves installation of crab cavities in colliders (such as KEK-B[60]) in order to compensate for beam-beam effects from a collision with a crossing angle. Because such colliders typically operate with a horizontal tune near a half-integer resonance, one may be particularly concerned about the effects of synchro-betatron coupling on stability and beam distribution. This problem was analyzed by Hoffstaetter and Chao [35] in which they find stop-band widths near linear resonances due to a single crab cavity and also due to dispersion at an $\mathrm{rf}$

\footnotetext{
${ }^{3}$ We should point out, however, that the existence of this formulation should not be seen as a strong argument for using the beam size over the inter-particle spacing for the maximum distance scatters to include. We have, in a sense, decided ahead of time that large distance scatters are relevant and can be included in an impulse approach.
} 
cavity.

Even if the crab cavity doesn't cause an instability, however, it may still have a strong effect on the beam distribution near a resonance. This issue led to the work contained in [29] and which also comprises a substantial part of this dissertation.

The effect of coupling near resonance has been well studied in the context of Hamiltonian perturbation theory. See [30] and [31]. In this approach, a distributed perturbation to the Hamiltonian is Fourier analyzed and slowly varying terms are extracted. For the case of linear perturbations such as linear coupling, a coupling parameter can be determined and the tunes found, particularly near sum and difference resonances.

In the presence of a damping and diffusion effect, a unique equilibrium distribution is reached. For the uncoupled case with synchrotron radiation, analytical expressions were given in [36]. A numerical algorithm to find this distribution for the general coupled case was given in [37]. A generalization of the synchrotron radiation integrals were given in $[32,33]$.

In reference [34], the authors find the equilibrium beam distribution near a betatron coupling resonance, using the Hamiltonian perturbation theory results of [31] to find the coupled Hamiltonian and resulting equilibrium beam distribution. We seek similar results for the synchro-betatron coupled case in addition to wanting to understand the beam distribution near integer and half-integer resonance. In [29] and this dissertation, we take a different approach to near resonance perturbation theory. We develop a new perturbation theory based directly on the one turn map, which for the linear case is a symplectic matrix. Near resonance, this is degenerate perturbation theory, with a close parallel to that of Quantum Mechanics ${ }^{4}$. We are able to derive the same results as found from the Hamiltonian approach in addition to being able to analyze the integer and half integer resonances caused by coupling which requires second order degenerate perturbation theory.

With this as introduction to the motivations of the work described in this dissertation, let us now return to a more general discussion of storage rings in order to

\footnotetext{
${ }^{4} \mathrm{~A}$ similar approach to the perturbation theory is taken in reference [66]. However, they do not consider the case of near resonance.
} 
describe our analytical framework.

In each type of storage ring, dipole magnets are used to bend the trajectory into a convenient shape. Quadrupole magnets provide transverse focussing about this orbit. The invention of alternating focussing quadrupole magnets allows stability in both horizontal and vertical directions. RF cavities are added to the ring to provide the acceleration of the beam and also for longitudinal focussing. There may also be additional dipole magnets in the form of insertion devices in electron storage rings for the purpose of additional cooling or for the production of coherent or incoherent radiation. There are also higher order multipole magnets which produce magnetic fields for other orbit related purposes. Although these elements act locally, for our purpose, it is their net global effect that is important. They can all be lumped together to produce a symplectic one-turn map. This map will depend on the position in the ring, but is determined by electric and magnetic fields throughout the ring, not just at the local position. The arrangement of these linear and nonlinear electromagnetic elements around the storage ring is called a lattice.

In addition to the planned fields due to the lattice, there are additional electric and magnetic fields the beam encounters around the ring. For one, it produces a self field, that acts on itself directly through what are termed space charge effects and intrabeam scattering, and indirectly through its interaction with the beam pipe and cavities. There will also be error fields, due to small discrepancies between the designed configuration of magnets and cavities and their actual configurations. Also, electrons and positrons can emit substantial synchrotron radiation when bent in their trajectories. This reduces their energy in addition to providing a noise source due to quantum fluctuations in the emitted photon energy.

One would like to know the effects of these additional processes on particle dynamics and beam distribution. We will assume that they are small, and can be treated as a perturbation to the design symplectic dynamics. These additional effects can be divided into symplectic and non-symplectic ones. We will take an asymmetric approach to these effects, treating the symplectic perturbations as perturbing the one-turn map, and the non-symplectic dynamics as causing local perturbation to the beam. 
The reason for this division comes from the fact that we assume that the dynamics is close to symplectic. We will also focus on the linear case in this thesis, in which the one-turn map is represented by a symplectic matrix. In this case, a beam in equilibrium can be described as a Gaussian function of the invariants of the one-turn map matrix [37]:

$$
f(\vec{z})=\frac{1}{\pi^{3}\left\langle g_{1}\right\rangle\left\langle g_{2}\right\rangle\left\langle g_{3}\right\rangle} \exp \left(-\frac{g_{1}}{\left\langle g_{1}\right\rangle}-\frac{g_{2}}{\left\langle g_{2}\right\rangle}-\frac{g_{3}}{\left\langle g_{3}\right\rangle}\right),
$$

where $g_{1,2,3}$ are quadratic invariants which we will write as $g_{a}=\vec{z}^{T} G_{a} \vec{z}$, with $a=$ $1,2,3 . \quad \vec{z}$ is the phase space vector and the ${ }^{T}$ indicates a transpose of the vector. $\left\langle g_{1,2,3}\right\rangle$ are the average values of the invariants over the beam distribution. They are related to the RMS eigen-emittances $\epsilon_{a}$ by $\left\langle g_{a}\right\rangle=2 \epsilon_{a}$.

Once these invariants have been determined, the distribution is only a function of three parameters, the $\left\langle g_{a}\right\rangle$. The non-symplectic dynamics locally change the emittances, but the form of the invariants are determined by the global one-turn map at a given location.

How do the non-symplectic dynamics change the beam distribution? We will assume the beam has a Gaussian distriubition, taking the form of (1.1). We can thus capture the effects of the non-symplectic dynamics in terms of the change to the second moments of the distribution which thereby change the $\left\langle g_{a}\right\rangle$. Let $\Sigma$ be the matrix of second moments. Then the non-symplectic dynamics will produce a local $d \Sigma / d s$ where $s$ is the position along the closed orbit trajectory. The change in the emittance around the ring is then given by

$$
\Delta\left\langle g_{a}\right\rangle=\oint d s \operatorname{Tr}\left[G_{a} \frac{d \Sigma}{d s}\right]
$$

This equation is the basis for our approach to the subject and captures the distinction between global symplectic dynamics and local non-symplectic dynamics: $G_{a}$ is the invariant of the global one-turn map at that position, and $d \Sigma / d s$ is the local change in the distribution due to the non-symplectic effects. The local change in the global invariant is then integrated around the ring to yield Eq. (1.2) for the total change in 
emittance.

So we see that there are two pieces to this approach to beam dynamics in a storage ring. The first involves finding the global one-turn map and the corresponding invariants. The second involves finding the local effect on the second moments due to all relevant non-symplectic processes. This interaction between local and global is one of the distinctive features of storage ring physics. For example, particles emitting synchrotron radiation undergo diffusion primarily in energy. However, due to dispersion which causes the transverse invariant to contain an energy component, the transverse emittance grows. More generally, diffusion or damping in one direction can, through global coupling, cause growth in other directions which could not be predicted by only looking at the local dynamics. As another example of this which we will encounter, the non-symplectic process of intrabeam scattering causes growth in the RMS momenta of the beam in such a way that energy in the beam frame is conserved. When energy conservation is described in terms of the global invariants, interesting effects arise, such as lack of an equillibrium, even in the smooth approximation when above the transition energy. Further, when one goes beyond the smooth approximation, using the locally varying global invariants, one finds that no equilibrium is possible with just IBS, even below transition. This is again an application of this interplay between local and global dynamics in storage rings ${ }^{5}$.

Stochastic effects can be included through the Fokker-Planck equation $[2,39,40]$ which gives the evolution of the single particle probability distribution which can be reinterpreted as the beam distribution itself. This is not as general a starting point as it may appear. It assumes that correlations between particles can be ignored ${ }^{6}$. Further, it is assumed that the stochastic kicks are small ${ }^{7}$. We mention these assumptions to bring attention to the fact that it is by no means obvious that the Fokker-Planck framework is adequate for describing the effects of IBS. In fact, this question is connected to the question of locality and $b_{\max }$ in the Coulomb Logarithm. Refer to the galactic dynamics literature (e.g. [2, 3, 4]) for more on this point. We will

\footnotetext{
${ }^{5}$ See [38] for further musings on this global/local philosophical issue.

${ }^{6}$ For a more general framework, see a discussion of the BBGKY hierarchy in e.g. [41]

${ }^{7}$ If one does not make this assumption, but still uses a single particle distribution function, this is the Master Equation (again, see e.g. [41]).
} 
here assume that the Fokker-Planck equation (or equivalent single particle stochastic differential equation) applies, taking the framework as seriously as possible. Indeed, the ability to do measurement of beam evolution due to IBS means that perhaps some of these old controversies can finally be put to rest ${ }^{8}$. But doing so requires both very careful measurement, and very careful understanding of assumptions going into the modeling analysis.

If we are only interested in Gaussian beams, as we will be in this dissertation, only the evolution of the second moments is required, as stated in Eq. (1.2). Then, one can use a single particle stochastic differential equation as the starting point to derive such moment evolution. In the case that non-Gaussian evolution were considered, the Fokker-Planck equation would be a more convenient tool. However, we will not consider this interesting question further. See refs. $[5,6,7,8,9]$. In the case that the distribution has substantial tails, but one still wants to use a Gaussian analysis, ref. [62] should be considered.

This thesis is organized as follows. In Chapter 2, we will give the details of the general formalism described in this introduction. The invariants are defined from the one turn map, and the distribution is described in terms of the invariants and their average values, or emittances. Given additional non-symplectic processes, the emittances will evolve, and we write down these evolution equations. We assume an uncoupled starting point and will consider coupling and non-symplectic dynamics as perturbations. We here give the details of the uncoupled symplectic starting point. We give the uncoupled invariants and transfer matrix in terms of the Courant-Snyder lattice parameters and dispersion functions. We give approximate lattice parameters for a smooth ring, the simple smoothed-out model replacing the actual ring with varying local properties.

In Chapter 3 we consider how linear coupling affects the symplectic dynamics. We develop a perturbation theory based on the one turn map. We focus on the case of being near a linear resonance where small coupling can cause large effects. Being on a resonance implies a degeneracy of the one-turn map, and we will thus require

\footnotetext{
${ }^{8}$ Computer simulation can also be of help here. However, the adequacy of macroparticle models which are inevitable for $10^{9}$ particles is uncertain and under investigation (see e.g. [42] for some recent work in this area).
} 
degenerate perturbation theory. We give analytical expressions for the perturbed eigenvalues by which questions of stability can be explored, and also the coupled invariants which determine the beam distribution. We explore the example of transverse coupling caused by skew quadrupole magnetic fields. We also consider the case of synchro-betatron coupling, and we give detailed results for the cases of dispersion at an RF cavity and a single crab cavity.

In Chapter 4, we add non-symplectic dynamics in the form of constant damping and diffusion matrices. Combined with linear symplectic dynamics, there is a unique Gaussian equilibrium beam distribution. This distribution can be approximately written as a function of the invariants of the one turn map, and we focus on this approximation which is typically quite good. In this case, we can define damping and diffusion coefficients for the eigen-invariants which give the equilibrium emittances. We consider the case of synchrotron radiation emitted in bending magnets in electron storage rings giving the explicit expressions for the local damping and diffusion matrices. We then derive the equilibrium emittances, which are well-known from the work of Sands [36].

In Chapter 5, we combine the results of the third and fourth chapters, giving expressions for the damping and diffusion coefficients for the coupled eigen-invariants near each of the linear coupling resonances. Near difference resonances we find that one of the damping coefficients can become negative, when the symplectic dynamics are still stable. This "anti-damping" indicates a slow instability, caused by the interplay of the damping with the structure of the invariants. This phenomenon can be seen in the "uncoupled" results of Chapter 4 as well. When the damping partition number is too large, an instability results. In this context dispersion in fact couples the transverse and longitudinal invariants and damping coefficients together. Our formalism shows that anti-damping is a more general effect, and occurs as a sum resonance is approached. Near an integer and half-integer resonance, we also derive the effect on the damping and diffusion coefficients. We find that the damping coefficients are not affected much by the resonance. However, the diffusion coefficients are affected, blowing up as the instability is approached.

In Chapter 6, we consider the case of non-constant damping and diffusion matrices. 
The example we explore is intrabeam scattering. In this case, we find that it is not useful to define global damping and diffusion coefficient for the eigen-invariants, although it can be done. Because a beam filling up larger phase space will have less diffusion and damping, there is a fundamental difference from the constant case in which diffusion is constant, and the damping effect increases with increased phase space volume. Instead, the local emittance growth can be directly computed. There are a variety of existing formulations of intrabeam scattering theory. Bjorken-Mtingwa and Piwinski find the emittance evolution directly, not breaking the scattering down in a damping piece and a diffusion piece. The damping and diffusion can combined together to find the evolution of the beam second moment and resulting emittance change, which is equivalent to the B-M and Piwinski results. The approach we take is most similar to the Rosenbluth Potentials [10], however we provide a new derivation of the diffusion and damping coefficients. The standard approaches involve a parameter called the Coulomb logarithm. Although not stated explicitly in most derivations, this parameter results from an assumption of spatial homogeneity (specifically involving impact parameters of colliision) of the beam. We formulate the problem in such a way that the real spatial distribution is involved and so no divergence occurs. We find that using the minimum beam size as a cut-off gives an excellent approximation to our more exact results in most cases. However, near to an equilibrium there can be substantial differences. We explore an example of bunch length growth in RHIC due to IBS and show that there is a small but possibly measurable difference between our equations and those of Bjorken-Mtingwa. We find that it is plausible that our equations provide a better fit to the data. Next, we survey the existing literature on IBS, describing the various ways in which the integrals can be approximated and under which conditions these approximations apply. Finally, we address the question of equilibrium, asking whether an equilibrium can occur when IBS is the only damping and diffusion mechanism. We confirm a result of Piwinski's, that below transition such an equilibrium can occur, while above transition it cannot. This result, however, is only valid in the smooth approximation. We find in a real non-smooth ring, even below transition, no equilibrium is possible, although the equilibrium predicted by the smooth approximation is approximately valid. The smooth approximation results can be understood 
in terms of Piwinski's invariant, a quantity conserved by IBS. It is basically energy conservation rewritten in terms of the invariants.

Chapter 7 combines the IBS results of Chapter 6 with coupling and synchrotron radiation discussed earlier. We give explicit expressions for the coupled matrices needed to compute the emittance evolution and give an example of $\mathrm{x}-\mathrm{y}$ coupling in the ATF at KEK. Finally we consider how Piwinski's invariant is affected in the presence of synchro-betatron coupling, finding that even below transition, with strong enough coupling, there is still no equilibrium even in the smooth approximation. 


\section{Chapter 2}

\section{General Overview}

Storage ring dynamics involves interaction between local and global dynamics. If we ignore non-linearity, the global dynamics is described by a one-turn map matrix, $M$. The one-turn map will have three independent invariants associated with it. Any beam distribution that is a function of these invariants will be stationary with respect to $M$. The presence of weak damping/diffusion does two things. First, it tends to cause the distribution to be a Gaussian function. We write a general normalized Gaussian distribution as

$$
f(\vec{z})=\frac{1}{(2 \pi)^{3} \sqrt{\operatorname{det}\left(\mathbb{M}^{-1}\right)}} e^{-\frac{1}{2} \vec{z}^{T} \mathbb{M} \vec{z}}
$$

In particular, the distribution will be a function of the invariants of $M$, which implies

$$
f(\vec{z})=\frac{1}{\pi^{3}\left\langle g_{1}\right\rangle\left\langle g_{2}\right\rangle\left\langle g_{3}\right\rangle} \exp \left(-\frac{g_{1}}{\left\langle g_{1}\right\rangle}-\frac{g_{2}}{\left\langle g_{2}\right\rangle}-\frac{g_{3}}{\left\langle g_{3}\right\rangle}\right)
$$

Here, the $g_{1,2,3}$ are quadratic invariants which we will write as $g_{a}=\vec{z}^{T} G_{a} \vec{z}$, with $a=1,2,3, \vec{z}$ being the phase space vector and the ${ }^{T}$ indicating a transpose of the vector. $\left\langle g_{1,2,3}\right\rangle$ are the average values of the invariants over the beam distribution. The second moment matrix, $\Sigma$ is given by $\mathbb{M}^{-1}$, and using (2.2), we can show that

$$
\Sigma=-J\left(\sum_{a}\left\langle g_{a}\right\rangle G_{a}\right) J
$$


with $J$ given in Eq. (2.8).

Further, the diffusion and damping cause a slow variation of $\left\langle g_{a}\right\rangle$. To find this evolution, we consider some non-symplectic process that causes a local change in the beam second moments $\Sigma_{j k}=\left\langle z_{j} z_{k}\right\rangle$. The net change in the $\left\langle g_{a}\right\rangle$ is then given by integrating the corresponding change in the invariant around the ring:

$$
\Delta\left\langle g_{a}\right\rangle=\oint d s \operatorname{Tr}\left[G_{a} \frac{d \Sigma}{d s}\right]
$$

with $\operatorname{Tr}$ representing the trace of a matrix. Thus, as we see, the non-symplectic process acts locally on the moments, causing changes in the invariants which are global quantities. Let us briefly consider the transformation properties of this equation. Let us suppose we apply a canonical transformation to phase space in the form of a symplectic matrix $\mathcal{N}$, and set $\vec{z}=\mathcal{N} \overline{\vec{z}}$. Then the moment matrix and the invariant matrix transform as $\Sigma=\left\langle\vec{z} \vec{z}^{T}\right\rangle=\mathcal{N} \Sigma \mathcal{N}^{T}$ and $\bar{G}_{a}=\mathcal{N} G_{a} \mathcal{N}$ so that

$$
\operatorname{Tr}\left[G_{a} \frac{d \Sigma}{d s}\right]=\operatorname{Tr}\left[\bar{G}_{a} \frac{d \bar{\Sigma}}{d s}\right]
$$

We will find two reasons to use this result. First, dispersion is a form of synchrobetatron coupling, coupling together transverse and longitudinal dynamics. However it is unavoidable in storage rings and we therefore would like to work it into our formalism at the beginning. We do this by using betatron coordinates that uncouple the dynamics. In this case, the matrix $\mathcal{N}$ is the dispersion matrix $\mathcal{B}$ which we give later. Using this transformation will simplify the form of the invariants, but we must remember to compute $\Sigma$ using these same coordinates. For the case of intrabeam scattering, one finds an additional reason to apply such a transformation. The effect of intrabeam scattering is most easily worked out in the beam frame. Thus, if we let $\mathcal{N}$ be a Lorentz transformation matrix, $L$, we can relate the second moment changes in the beam frame to the lab frame emittance changes.

The calculation implied by Eq. (2.4) will organize our discussion in this chapter and in fact our general approach. It will be useful at times, when in the midst of a detailed calculation, to return to this equation to remind ourselves of how that 
detail fits into the bigger picture. In this chapter, we will start with a discussion of the computation of the invariants $G_{a}$ from the one-turn map $M$, giving explicit expressions for the uncoupled case. Next we discuss the change in the second moments from the local non-symplectic dynamics.

\subsection{Linear Dynamics and Invariants}

Consider a general electron storage ring with a given linear lattice. Ignoring the damping and diffusion effects due to synchrotron radiation, we can describe the dynamics at any position $s$ in terms of a one-turn map $M$ :

$$
\vec{z}_{s+C}=M(s) \vec{z}_{s}
$$

where $C$ is the circumference of the ring and where $\vec{z}$ is our phase space coordinate vector (column matrix). Our coordinates are $\left(x, x^{\prime}, y, y^{\prime}, z, \delta\right)$ where $x$ and $z$ are the particle's horizontal and longitudinal displacements relative to the beam center, $x^{\prime}=p_{x} / p_{z}$ is the slope of the particle's motion in $x$, and $\delta=\left(p_{z}-P_{0}\right) / P_{0}$ where $P_{0}$ is the design reference momentum.

The matrix $M$ is symplectic [22] which means

$$
M^{T} J M=J,
$$

where a superscript $T$ means taking the transpose of a matrix, and $J$ is the symplectic inner product matrix

$$
J=\left(\begin{array}{cccccc}
0 & 1 & 0 & 0 & 0 & 0 \\
-1 & 0 & 0 & 0 & 0 & 0 \\
0 & 0 & 0 & 1 & 0 & 0 \\
0 & 0 & -1 & 0 & 0 & 0 \\
0 & 0 & 0 & 0 & 0 & 1 \\
0 & 0 & 0 & 0 & -1 & 0
\end{array}\right)
$$


The six eigenvectors and eigenvalues of $M$ satisfy

$$
M v_{k}=\lambda_{k} v_{k}
$$

The index $k$ runs over $\pm 1, \pm 2, \pm 3$. We normalize the eigenvectors such that

$$
v_{j}^{\dagger} J v_{k}=i \operatorname{sgn}(j) \delta_{j k}
$$

where $\operatorname{sgn}(j)$ is 1 for $j>0$ and -1 for $j<0$, and ${ }^{\dagger}$ means taking the complex conjugate and transpose of a matrix (or vector). This normalization condition suggests the definition of an upper indexed object

$$
v^{j} \equiv-i \operatorname{sgn}(j) v_{j}^{\dagger} J
$$

The normalization condition (2.10) then reads

$$
v^{j} v_{k}=\delta_{j k}
$$

We refer to an eigenvector $v_{k}$ with $k>0$ as a positive mode eigenvector and one with $k<0$ as a negative mode eigenvector. Note that this property of being a positive or negative mode is intrinsic to a given eigenvector and is not a property of the normalization: multiplying by a constant cannot convert a positive mode eigenvector into a negative mode, and vice versa. The positive and negative modes are related as follows,

$$
v_{-k}=i v_{k}^{*}, \quad v^{-k}=-i v^{k *}
$$

When $M$ describes stable motion in the storage ring, the eigenvalues $\lambda_{k}$ can be expressed as

$$
\lambda_{k}=e^{i \mu_{k}},
$$

with $\mu_{k}$ a real quantity. We refer to $\mu_{k}$ as the eigen-phase advance, with $\mu_{k}=2 \pi \nu_{k}$ where $\nu_{k}$ is the eigen-tune. The positive and negative phase advances are related to 
each other as

$$
\mu_{-k}=-\mu_{k}
$$

From the $v_{k}$ 's, we construct a matrix using them as columns,

$$
U=\left(\begin{array}{llllll}
v_{1} & v_{-1} & v_{2} & v_{-2} & v_{3} & v_{-3}
\end{array}\right)
$$

Given the normalization, one can show that $U$ is symplectic. We can thus consider $U$ as a (complex) canonical transformation that diagonalizes ${ }^{1} M$. In particular

$$
\begin{aligned}
U^{-1} M U & =e^{\Lambda} \\
& =\left(\begin{array}{cccccc}
e^{i \mu_{1}} & 0 & 0 & 0 & 0 & 0 \\
0 & e^{-i \mu_{1}} & 0 & 0 & 0 & 0 \\
0 & 0 & e^{i \mu_{2}} & 0 & 0 & 0 \\
0 & 0 & 0 & e^{-i \mu_{2}} & 0 & 0 \\
0 & 0 & 0 & 0 & e^{i \mu_{3}} & 0 \\
0 & 0 & 0 & 0 & 0 & e^{-i \mu_{3}}
\end{array}\right) .
\end{aligned}
$$

Let us now discuss the invariants of $M$. Let $g=\vec{z}^{T} G \vec{z}$ be a quadratic invariant of $M$, where $G$ is a symmetric matrix. To be an invariant, $g(s)$ must satisfy the condition $g(s)=g(s+C)$ for all $\vec{z}$, or

$$
M^{T} G M=G
$$

We can in fact use the $g_{a}$ and additional 3 conjugate angle quantities $\phi_{a}$ as coordinates for phase space. These are the well known action-angle coordinates. From the definition of the invariants, it is easy to see that if the distribution is a function only of the $g_{a}$ and not the $\phi_{a}$, then it will be stationary with respect to the symplectic dynamics. Evolution occurs in the $\left\langle g_{a}\right\rangle$ while the $\phi_{a}$ evolution is washed out. Note

\footnotetext{
${ }^{1}$ Another useful canonical transformation involves taking $\sqrt{2}$ times the real and imaginary parts of the $v_{k}$ 's for the columns of $U$. This is a real canonical transformation and results in a block diagonal $M$ with blocks given by rotation matrices. Thanks to Y. Cai of SLAC for pointing this out. It is the same as that given in [23]. We discuss this transformation in Appendix A, but otherwise do not make use of it in this work.
} 
that linear combinations of invariants are still invariants. In the non-degenerate case, there are 3 linearly independent invariants. Given $U$, there is a natural choice for these three invariants. We express them as

$$
g_{a}=\vec{z}^{T} G_{a} \vec{z}, \quad G_{a}=J U H_{a} U^{T} J, \quad a=1,2,3
$$

with

$$
\begin{array}{rlrl}
H_{1} & =\left(\begin{array}{ccc}
i \sigma_{x} & 0 & 0 \\
0 & 0 & 0 \\
0 & 0 & 0
\end{array}\right), & H_{2}=\left(\begin{array}{ccc}
0 & 0 & 0 \\
0 & i \sigma_{x} & 0 \\
0 & 0 & 0
\end{array}\right), \\
H_{3}=\left(\begin{array}{ccc}
0 & 0 & 0 \\
0 & 0 & 0 \\
0 & 0 & i \sigma_{x}
\end{array}\right), & \sigma_{x}=\left(\begin{array}{ll}
0 & 1 \\
1 & 0
\end{array}\right) .
\end{array}
$$

One can verify that these are invariants by using (2.18), and more details are given in Appendix A. These invariants ${ }^{2}$ can also be written directly in terms of the eigenvectors as

$$
G_{a}=-J\left(v^{*} v^{T}+v v^{\dagger}\right) J
$$

From this expression, we note two properties of the invariants. First, they are real quantities, and second, they are independent of the overall phase of the eigenvector $v_{a}$. Further, there is no need to allow $a$ to run over negative values. If we do so, Eq. (2.21) shows that $G_{-a}=G_{a}$. That is, the invariants determined by the negative mode eigenvectors are identical to those from the corresponding positive mode. This is a manifestation of the relationship between $v_{k}$ and $v_{-k}$.

The invariants $g_{a}$, expressed in terms of normalized eigenvectors in (2.19) and (2.21), turn out to be the action variables often used in perturbation theory in classical systems. To see that, let us express the one-turn map as

$$
M=e^{J S}
$$

\footnotetext{
${ }^{2}$ Note that we use the term invariant for both $g_{a}$ and $G_{a}$, although only the $g_{a}$ are actually invariant with respect to the particle motion.
} 
In this form, $S$ has the physical meaning that the effective Hamiltonian describing the one-turn motion around the observation point $s$ is given by $H=\frac{1}{2} \vec{z}^{T} S \vec{z}$. Then using

$$
\begin{gathered}
S=-J U \Lambda U^{-1}, \quad \Lambda=\left(\begin{array}{ccc}
i \mu_{1} \sigma_{z} & 0 & 0 \\
0 & i \mu_{2} \sigma_{z} & 0 \\
0 & 0 & i \mu_{3} \sigma_{z}
\end{array}\right) \\
\text { and } \quad \sigma_{z}=\left(\begin{array}{cc}
1 & 0 \\
0 & -1
\end{array}\right),
\end{gathered}
$$

it follows that

$$
S=\mu_{1} G_{1}+\mu_{2} G_{2}+\mu_{3} G_{3}
$$

The one-turn effective Hamiltonian is therefore given by

$$
H=\frac{1}{2}\left(\mu_{1} g_{1}+\mu_{2} g_{2}+\mu_{3} g_{3}\right)
$$

For the transverse dimension, the $g_{a}$ corresponding to the betatron oscillations reduces to the Courant-Snyder invariant [22] in the uncoupled case. ${ }^{3}$

Now, suppose that the distribution of electrons in the storage ring is given as a function of the invariants $g_{a}$. In particular, let it have a Gaussian distribution given by Eq.(2.2). In terms of matrices $G_{a}$, the second moments of the distribution

$$
\left\langle z_{i} z_{j}\right\rangle=\Sigma_{i j}
$$

are given by

$$
\Sigma=-\frac{1}{2}\left\langle g_{1}\right\rangle J G_{1} J-\frac{1}{2}\left\langle g_{2}\right\rangle J G_{2} J-\frac{1}{2}\left\langle g_{3}\right\rangle J G_{3} J,
$$

which we derive in Appendix A.

\footnotetext{
${ }^{3}$ For the sake of this agreement, we have not included a factor of $\frac{1}{2}$ in the definition of the $g_{a}$, Eq.(2.19).
} 


\subsection{Unperturbed Case}

A typical storage ring is designed to be planar and uncoupled and this will be our starting point. We will use betatron coordinates, defined by $\vec{z}_{\beta}=\mathcal{B} \vec{z}$. The dispersion matrix $\mathcal{B}$ is given by

$$
\mathcal{B}=\left(\begin{array}{cccccc}
1 & 0 & 0 & 0 & 0 & -\eta_{x} \\
0 & 1 & 0 & 0 & 0 & -\eta_{x}^{\prime} \\
0 & 0 & 1 & 0 & 0 & -\eta_{y} \\
0 & 0 & 0 & 1 & 0 & -\eta_{y}^{\prime} \\
\eta_{x}^{\prime} & -\eta_{x} & \eta_{y}^{\prime} & -\eta_{y} & 1 & 0 \\
0 & 0 & 0 & 0 & 0 & 1
\end{array}\right)
$$

with $\eta_{x, y}$ the horizontal and vertical dispersion function and $\eta_{x, y}^{\prime}$ the derivative with respect to the position on the orbit $s$.

Then the linear one-turn map for the ring at $s$ is of the form

$$
M_{\text {uncoupled }}=\left(\begin{array}{ccc}
M_{x} & 0 & 0 \\
0 & M_{y} & 0 \\
0 & 0 & M_{z}
\end{array}\right) \text {. }
$$

Where $M_{x, y, z}$ are symplectic $2 \times 2$ matrices. Since they are symplectic, following Courant and Snyder [22], we can write them in the form

$$
M_{u}=\cos \mu_{u} I+\sin \mu_{u} J_{u}=e^{\mu_{u} J_{u}}, J_{u}=\left(\begin{array}{cc}
\alpha_{u} & \beta_{u} \\
-\gamma_{u} & -\alpha_{u}
\end{array}\right)
$$

where $u$ stands for $x, y$, or $z$. Here, $\beta_{x, y}, \alpha_{x, y}=-2 \beta_{x, y}^{\prime}$, and $\gamma_{x, y}=\frac{1+\alpha_{x, y}^{2}}{\beta_{x}}$ are the usual horizontal and vertical Courant-Snyder lattice parameters. They are periodic with period $C$; e.g. $\beta_{x}(s+C)=\beta_{x}(s)$. Note that adding integer multiples of $2 \pi$ to $\mu_{x}$ and $\mu_{z}$ does not change the one turn map. We will thus, except where otherwise noted, assume that an appropriate multiple has been added (subtracted) so that

$$
\mu_{x, z} \in[-\pi, \pi]
$$


To find the corresponding quantities for $z$, let us consider a model storage ring with one RF cavity. The transfer matrix for the RF cavity can then be written as

$$
T_{\text {cav }}=\left(\begin{array}{cc}
1 & 0 \\
\frac{r}{a} & 1
\end{array}\right)
$$

where $r=4 \sin ^{2} \frac{\mu_{z}}{2}$. The transfer matrix from the RF cavity to another position $s$ is

$$
T_{z \rho}(s)=\left(\begin{array}{cc}
1 & -a \check{\alpha} \\
0 & 1
\end{array}\right)
$$

The subscript tells us that this is the longitudinal transfer matrix taking bends into account. Here, $a=C \eta_{s}$ with

$$
\eta_{s}=\alpha_{c}-\frac{1}{\gamma^{2}}
$$

is the momentum slip factor with $\alpha_{c}$ the momentum compaction factor, and

$$
\check{\alpha}=\frac{1}{a} \int_{s_{c}}^{s} \frac{\eta\left(s^{\prime}\right)}{\rho\left(s^{\prime}\right)} d s^{\prime},
$$

with $s_{c}$ the position of the RF cavity, $\eta$ the dispersion and $\rho$ the dipole bending radius. We note that $\check{\alpha}$ depends on the two positions $s_{c}$ and $s$ and ranges from 0 (when $s=s_{c}$ ) to 1 (when $s=s_{c}+C$ ). We call $\alpha_{c} \check{\alpha}$ the partial momentum compaction. The one-turn map at an arbitrary position $s$ is then

$$
M_{z}=\left(\begin{array}{cc}
1-r \check{\alpha} & -a[1+r \check{\alpha}(\check{\alpha}-1)] \\
\frac{r}{a} & 1-r+r \check{\alpha}
\end{array}\right) .
$$

One can verify that the eigenvalues of $M_{z}$ are indeed $e^{ \pm i \mu_{z}}$. Comparing the two different forms for $M_{z}$, we identify

$$
\begin{aligned}
\beta_{z} & =-\frac{a[1+\check{\alpha} r(\check{\alpha}-1)]}{\sin \mu_{z}}, \\
\gamma_{z} & =-\frac{r}{a \sin \mu_{z}} \\
\alpha_{z} & =(1-2 \check{\alpha}) \tan \frac{\mu_{z}}{2} .
\end{aligned}
$$


The synchrotron phase advance per turn $\mu_{z}$ is typically small. Taking lowest order in $\mu_{z}$ we get

$$
\beta_{z}=-\frac{a}{\mu_{z}}, \quad \gamma_{z}=-\frac{\mu_{z}}{a}, \quad \alpha_{z}=\frac{\mu_{z}}{2}(1-2 \check{\alpha}) .
$$

Note that in order to preserve the symplectic requirement that $\beta_{z} \gamma_{z}=1+\alpha_{z}^{2}$, we have to keep higher order in $\mu_{z}$ in $\beta_{z}$ and $\gamma_{z}$.

We require that $\beta_{z}$ and $\gamma_{z}$ be positive. This means that $\mu_{z}$ must be negative (assuming $a>0$, which is true above transition). Thus, designating the usual (positive) synchrotron phase as $\mu_{s}$, we have $\mu_{z}=-\mu_{s}$. In terms of $\mu_{s}$ then,

$$
\beta_{z}=\frac{a}{\mu_{s}}, \quad \gamma_{z}=\frac{\mu_{s}}{a}, \quad \alpha_{z}=\frac{-\mu_{s}}{2}(1-2 \check{\alpha})
$$

Given the general form (2.30), we can express the eigenvectors of $M_{\text {uncoupled }}$ as

$$
v_{x}=\frac{1}{\sqrt{2}}\left(\begin{array}{c}
\sqrt{\beta_{x}} \\
\frac{i-\alpha_{x}}{\sqrt{\beta_{x}}} \\
0 \\
0 \\
0 \\
0
\end{array}\right), \quad v_{y}=\frac{1}{\sqrt{2}}\left(\begin{array}{c}
0 \\
0 \\
\sqrt{\beta_{y}} \\
\frac{i-\alpha_{y}}{\sqrt{\beta_{y}}} \\
0 \\
0
\end{array}\right) \quad v_{z}=\frac{1}{\sqrt{2}}\left(\begin{array}{c}
0 \\
0 \\
0 \\
0 \\
\sqrt{\beta_{z}} \\
\frac{i-\alpha_{z}}{\sqrt{\beta_{z}}}
\end{array}\right)
$$

with corresponding eigenvalues $e^{i \mu_{x}}, e^{i \mu_{y}}$ and $e^{i \mu_{z}}$, which can be checked by direct multiplication. The above $v_{x}$ and $v_{z}$ are positive modes, and the corresponding negative modes are $v_{-x}=i v_{x}^{*}$ and $v_{-z}=-i v_{z}^{*}$. Using the notation in (2.11), we can express the normalization as $v^{x} v_{x}=v^{z} v_{z}=v^{-x} v_{-x}=v^{-z} v_{-z}=1$ and all other combinations give 0 . To be explicit, because $v_{x}$ is a positive mode (likewise $v_{z}$ ), $v^{x}=-i v_{x}^{\dagger} J$. 
From these eigenvectors, we find the invariants:

$$
\begin{aligned}
G_{x} & =\left(\begin{array}{cccccc}
\gamma_{x} & \alpha_{x} & 0 & 0 & 0 & 0 \\
\alpha_{x} & \beta_{x} & 0 & 0 & 0 & 0 \\
0 & 0 & 0 & 0 & 0 & 0 \\
0 & 0 & 0 & 0 & 0 & 0 \\
0 & 0 & 0 & 0 & 0 & 0 \\
0 & 0 & 0 & 0 & 0 & 0
\end{array}\right), \\
G_{y} & =\left(\begin{array}{llllll}
0 & 0 & 0 & 0 & 0 & 0 \\
0 & 0 & 0 & 0 & 0 & 0 \\
0 & 0 & \gamma_{y} & \alpha_{y} & 0 & 0 \\
0 & 0 & \alpha_{y} & \beta_{y} & 0 & 0 \\
0 & 0 & 0 & 0 & 0 & 0 \\
0 & 0 & 0 & 0 & 0 & 0
\end{array}\right), \\
G_{z} & =\left(\begin{array}{llllll}
0 & 0 & 0 & 0 & 0 & 0 \\
0 & 0 & 0 & 0 & 0 & 0 \\
0 & 0 & 0 & 0 & 0 & 0 \\
0 & 0 & 0 & 0 & 0 & 0 \\
0 & 0 & 0 & 0 & \gamma_{z} & \alpha_{z} \\
0 & 0 & 0 & 0 & \alpha_{z} & \beta_{z}
\end{array}\right) .
\end{aligned}
$$

For the $x$ direction, we find the standard Courant-Snyder invariant,

$$
g_{x}=\gamma_{x} x_{\beta}^{2}+2 \alpha_{x} x_{\beta} x_{\beta}^{\prime}+\beta_{x} x_{\beta}^{\prime 2},
$$

and likewise for $y$, where we recall that we are using the $\vec{z}_{\beta}$ betatron coordinates defined in Eq.(2.28).

We now consider how the eigenvectors transform around the ring. This is necessary because we will want to know how the damping and diffusion coefficients transform around the ring, and they depend on the local eigenvectors. The eigenvectors transform in the same way as the phase space coordinate $\vec{z}$ does, i.e. with the transfer matrix from position $s_{1}$ to position $s_{2}$. We express the transfer matrix in terms of the 
Courant-Snyder lattice functions and the phase advance from $s_{1}$ to $s_{2}, \psi_{x 12}$, which is related to $\beta_{x}(s)$ by

$$
\psi_{x 12}=\int_{s_{1}}^{s_{2}} \frac{d s}{\beta_{x}(s)}
$$

In betatron coordinates, the transfer matrix is given by

$$
T_{0 \beta}\left(s_{1} \rightarrow s_{2}\right) \equiv T_{12 \beta}=\left(\begin{array}{ccc}
R_{x} & 0 & 0 \\
0 & R_{y} & 0 \\
0 & 0 & R_{z}
\end{array}\right)
$$

with

$$
R_{z}=\left(\begin{array}{cc}
1 & -a \check{\alpha} \\
0 & 1
\end{array}\right)
$$

where $a \check{\alpha}$ is $C$ times the partial momentum compaction factor from $s_{1}$ to $s_{2}$, and for $R_{x}$ and $R_{y}$,

$$
\begin{aligned}
& R_{11}=\sqrt{\frac{\beta_{2}}{\beta_{1}}}\left(\cos \psi_{x 12}+\alpha_{1} \sin \psi_{x 12}\right), \\
& R_{12}=\sqrt{\beta_{1} \beta_{2}} \sin \psi_{x 12}, \\
& R_{21}=\frac{1}{\sqrt{\beta_{1} \beta_{2}}}\left[\left(\alpha_{1}-\alpha_{2}\right) \cos \psi_{x 12}-\left(1+\alpha_{1} \alpha_{2}\right) \sin \psi_{x 12}\right], \\
& R_{22}=\sqrt{\frac{\beta_{1}}{\beta_{2}}}\left(\cos \psi_{x}-\alpha_{2} \sin \psi_{x 12}\right),
\end{aligned}
$$

where $\beta_{1}=\beta_{x, y}\left(s_{1}\right)$, etc. When $s_{2}=s_{1}+C, \psi_{x, y 12}=\mu_{x, y}$.

We have not written the longitudinal transfer matrix in terms of $\beta_{z}, \alpha_{z}$, and $\gamma_{z}$ for reasons of convenience. We have assumed that we have not crossed an RF cavity from $s_{1}$ to $s_{2}$. In the case that we do, we need to also multiply by (2.32). To lowest order in $\mu_{s}$,

$$
\psi_{z 12}=\check{\alpha} \mu_{s}
$$

where $\check{\alpha}$ is integrated from $s_{1}$ to $s_{2}$. This can be derived by setting $R_{12}$ for the $z$ transformation $(-a \check{\alpha})$ equal to $\sqrt{\beta_{z 1} \beta_{z 2}} \sin \psi_{z 12}$. In the general case with multiple RF cavities, we would need to compute the resulting $\beta_{z}, \alpha_{z}$ and $\gamma_{z}$, but otherwise the formalism remains the same. Note, however, that we assume that the dispersion 
at each of the RF cavities is 0 in the computation of the $\alpha_{z}, \beta_{z}$, and $\gamma_{z}$. These are uncoupled lattice functions. We treat dispersion at an RF cavity as a perturbation and later give an example for the case of a single cavity.

The transfer map in betatron coordinates is related to the transfer map in real coordinates by

$$
T_{12 \beta}=\mathcal{B}_{2} T_{12} \mathcal{B}_{1}^{-1}
$$

where $\mathcal{B}_{1}$ and $\mathcal{B}_{2}$ are the betatron coordinate transformation matrices given in (2.28) involving the dispersion at $s_{1}$ and $s_{2}$ respectively.

Applying $T_{12 \beta}$ to the eigenvectors, we can show

$$
\begin{gathered}
T_{12 \beta} v_{x}\left(s_{1}\right)=e^{i \psi_{x 12}} v_{x}\left(s_{2}\right), \\
T_{12 \beta} v_{z}\left(s_{1}\right)=e^{i \psi_{z 12}} v_{z}\left(s_{2}\right),
\end{gathered}
$$

where $v_{x}\left(s_{2}\right)$ and $v_{z}\left(s_{2}\right)$ are expressions (2.40) with the lattice functions advanced to position $s_{2}$.

\subsection{Smooth Storage Ring}

It is sometimes useful to replace the real machine with varying lattice parameters with a smoothed out ring where all positions are equivalent. There are two ways in which one might define such a ring. First, one could specify some "typical" position in the ring. Then, one could simply assume that all places in the ring have the same one turn map. One could choose the typical position by replacing the lattice parameters with their average values.

Another way to define a smooth machine is to imagine physically spreading our the bending and focussing elements throughout the whole ring. Then the bending radius will be given by

$$
\rho_{\text {s.a. }}=R
$$


Setting $\beta_{x, y}$ to be constants such that the tunes remain $\nu_{x, y}$ give

$$
\begin{aligned}
\beta_{x, y} & =\frac{R}{\nu_{x, y}} \\
\beta_{z} & =\frac{R\left(\frac{1}{\nu_{x}^{2}}-\frac{1}{\gamma^{2}}\right)}{\nu_{z}} \\
\eta_{x} & =\frac{R}{\nu_{x}^{2}}
\end{aligned}
$$

where we have used the smoothed value of the momentum compaction

$$
\alpha_{c}=\frac{1}{\nu_{x}^{2}}
$$

\section{$2.4 \quad$ Non-symplectic process}

We now consider the effect of adding a small non-symplectic perturbation to the otherwise symplectic dynamics. We write this as ${ }^{4}$

$$
\frac{d z_{i}}{d t}=\left(J S_{l} \vec{z}\right)_{i}+\tilde{\xi}_{i}
$$

where $S_{l}$ is the local Hamiltonian matrix. We will later convert time derivatives into derivatives with respect to path length. Consider a time $\Delta t$ that is short relative to the symplectic dynamics, but long enough that the stochastic term $\hat{\xi}_{i}$ has acted many times. The value of $z_{i}$ after $\Delta t$ is then

$$
z_{i}(t+\Delta t)=z_{i}(t)+\left(J S_{l} \vec{z}\right)_{i} \Delta t+\Delta z_{i}
$$

where

$$
\Delta z_{i}=\int_{t}^{t+\Delta t} \hat{\xi}_{i}\left(t^{\prime}\right) d t^{\prime}
$$

\footnotetext{
${ }^{4}$ We assume that $\tilde{\xi}_{i}$ is entirely a non-symplectic process. In order to implement this in practicality, we will later have to subtract off the reversible components to the evolution, leaving an entirely irreversible process.
} 
From Eq. (2.58) we can find an equation for the evolution of the second moments of the beam distribution $\Sigma_{i j}$ :

$$
\frac{\Delta \Sigma_{i j}}{\Delta t}=\int d \vec{z} f(\vec{z})\left(\left(\left(J S_{l} z\right)_{i} z_{j}+\left(J S_{l} z\right)_{j} z_{i}\right)+z_{i} \tilde{b}_{j}+z_{j} \tilde{b}_{i}+\tilde{d}_{i j}\right)
$$

where

$$
\begin{aligned}
\tilde{b}_{i} & =\frac{\left\langle\Delta z_{i}\right\rangle_{W}}{\Delta t} \\
\tilde{d}_{i j} & =\frac{\left\langle\Delta z_{i} \Delta z_{j}\right\rangle_{W}}{\Delta t}
\end{aligned}
$$

The $b_{i}$ are known as the drift coefficients and contain damping effects and additional Hamiltonian effects. The $d_{i j}$ are the diffusion coefficients. We have averaged over $f$, the beam distribution, and also over $W$, the ensemble of possible events determining the process $\hat{\xi}$ between $t$ and $t+\Delta t$. We note that the diffusion and damping coefficients can depend on the phase space position $\vec{z}$.

Let us say a bit more about this averaging over the ensemble of events. We consider the stochastic process $\hat{\xi}_{i}$ as composed of $n$ short kicks so that

$$
\Delta z_{i}=\sum_{j=1}^{n} \delta z_{i}^{(j)}
$$

where the $\delta z_{i}^{(j)}$ are the kicks and $n$ is the expected number of events between $t$ and $t+\Delta t$. The damping and diffusion coefficients are then given by

$$
\begin{aligned}
\tilde{b}_{i} & =\frac{\left\langle\sum_{j} \delta z_{i}^{(j)}\right\rangle_{W}}{\Delta t} \\
\tilde{d}_{i j} & =\frac{\left\langle\sum_{k, l} \delta z_{i}^{(k)} \delta z_{j}^{(l)}\right\rangle_{W}}{\Delta t}
\end{aligned}
$$

The double sum in the diffusion coefficient can be broken into terms with the same 
and different summation index:

$$
\tilde{d}_{i j}=\frac{\left\langle\sum_{k} \delta z_{i}^{(k)} \delta z_{j}^{(k)}\right\rangle_{W}}{\Delta t}+\frac{\left\langle\sum_{k, l(k \neq l)} \delta z_{i}^{(k)} \delta z_{j}^{(l)}\right\rangle_{W}}{\Delta t}
$$

Let us consider a single term in the second bracketed quantity:

$$
\left\langle\delta z_{i}^{(k)} \delta z_{j}^{(l)}\right\rangle_{W}
$$

This quantity is an ensemble average of a product of kicks at two different times. We claim that this quantity is negligible because the two kicks are uncorrelated. However, it is not entirely true that the two kicks are uncorrelated. We have included both damping and diffusion in the same process and so in the case that the damping doesn't vanish, the kicks are not uncorrelated, but have an average value equal to the damping. However, since we only keep the damping effect to lowest order, this term is higher order in the damping and we thus disregard these terms.

Let us now suppose that $\delta z_{i}$ depends on some parameter $\Phi$, and $\Phi$ is distributed according to a normalized distribution $g(\Phi)$. Then, instead of averaging using the more general quantity $W$, we write the diffusion and damping coefficients as

$$
\begin{aligned}
\tilde{b}_{i} & =\dot{n} \int d \Phi g(\Phi) \delta z_{i}(\Phi) \\
\tilde{d}_{i j} & =\dot{n} \int d \Phi g(\Phi) \delta z_{i}(\Phi) \delta z_{j}(\Phi)
\end{aligned}
$$

where $\dot{n}=n / \Delta t$ is the expected event rate. In the diffusion coefficient, we have used the fact that the kicks are uncorrelated to reduce the double sum (integral) to a single one. In the case of synchrotron radiation, $\Phi$ will be the frequency of the emitted photon, and $g(\Phi)$ the normalized spectrum. For intrabeam scattering, $\Phi$ will be $\vec{z}_{2}$, the phase space position of the second particle with which the given particle is scattering, and $g$ will be the distribution of particles which undergo the scattering process within the time $t$ and $t+\Delta t$. In each case, we must normalize $g(\Phi)$ so that its integral is 1 . 
We have expressed Eqs. (2.67) and (2.68) in terms of general indices $i, j$. This allows us to compute these in any coordinates. In physical coordinates, however, only the momenta can change instantaneously and so only the momentum indices are non-zero.

In the case where there is constant damping and diffusion, we have

$$
\begin{aligned}
\tilde{b}_{i} & =B_{i k} z_{k} \\
\tilde{d}_{i j}=D_{i j} & =\text { constant }
\end{aligned}
$$

where we recall that for physical coordinates, $i$ and $j$ should just run over the momentum indices. We could also write the damping coefficients as

$$
B_{a b}=\frac{\partial \tilde{b}_{a}}{\partial p_{b}}
$$

with $a$ and $b$ referring to momentum indices.

In this case of constant damping/diffusion, one can prove that the equilibrium resulting from combining linear symplectic dynamics with the additional irreversible process is a unique Gaussian distribution. If the distribution is indeed Gaussian, then it can be completely characterized by its second moments $\Sigma_{i j}=\left\langle z_{i} z_{j}\right\rangle$. Integrating Eq. (2.60), we find

$$
\Sigma(s+C)=M \Sigma(s) M^{T}+\int_{s}^{s+C} d s^{\prime} T_{s \rightarrow s^{\prime}}\left\langle z_{i} \tilde{b}_{j}+z_{j} \tilde{b}_{i}+\tilde{d}_{i j}\right\rangle_{f\left(s^{\prime}\right)} T_{s \rightarrow s^{\prime}}^{T}
$$

Here $M(s)$ is the symplectic one-turn map matrix at position $s$ and $T_{s \rightarrow s^{\prime}}$ is the transfer matrix from $s$ to $s^{\prime}$. It is again symplectic. Indeed, $M(s)=T_{s \rightarrow s+C}$ where $C$ is the ring circumference. The first term of this moment evolution equation gives the symplectic evolution of the second moments and the integrated terms give the total effect on the moments from the irreversible process $W$. 


\subsection{Reversible/Irreversible Dynamics}

As written, the stochastic quantity $\tilde{\xi}$ could contain both reversible and irreversible parts. We would like to pull out the reversible parts and consider that as being part of the Hamiltonian evolution. Consider the change in the second moments due to drift and diffusion as given in Eq. (2.60):

$$
\frac{d \Sigma_{i j}}{d t}=\left\langle\tilde{b}_{i} z_{j}+\tilde{b}_{j} z_{i}+\tilde{d}_{i j}\right\rangle
$$

Now, $\tilde{b}_{i}$ and $\tilde{d}_{i j}$ depend on the phase space coordinate $\vec{z}$. The process of time reversal can be represented by reversing the direction of the momentum

$$
p_{a} \rightarrow-p_{a}
$$

For $d \Sigma / d t$ to be the same after time reversal, we require that the momentum indices of $b_{i}$ to be antisymmetric and those of $d_{i j}$ to be symmetric:

$$
\begin{gathered}
\tilde{b}_{a}(-\vec{p})=-\tilde{b}_{a}(\vec{p}) \\
\tilde{d}_{a b}(-\vec{p})=\tilde{d}_{a b}(\vec{p})
\end{gathered}
$$

where the $a$ and $b$ represent the momentum indices. See [69] for more discussion of this condition. One can see this more clearly when considering the Fokker-Planck equation, however the same logic applies starting from the single particle stochastic equation. We will use it to pick out the irreversible components of the drift and diffusion coefficients.

Eq. (2.75) suggests that we write

$$
\tilde{b}_{a}=B_{a b} p_{b}
$$

where $B_{a b}$ is independent of $\vec{z}$ to lowest order. Thus, the simplest situation is where $B_{a b}$ and $\tilde{d}_{a b}$ have no phase-space dependence. This will be considered in Chapter III and covers the case of synchrotron radiation. The more general case of $\vec{z}$ dependence 
is required for the case where the stochasticity comes from the particle interacting within the same beam as is the case with intrabeam scattering or with another case as with beam-beam diffusion. These cases are considered in Chapter V. 


\section{Chapter 3}

\section{Perturbation Theory Near Linear Resonances}

In Chapter 2, we laid out our general approach. We saw that there are two elements needed to find the beam distribution, the global invariants and local non-symplectic second moment evolution. In this section, we focus on the symplectic (reversible) dynamics through analysis of the one-turn map $M$. There are two pieces of information we seek from this analysis. Before asking about the beam distribution, we should first determine whether or not the single particle dynamics are stable. This can be found from the eigenvalues of $M$. Assuming symplectic stability, we would then like to understand the beam distribution. For this, we require the invariants, which can be constructed from the eigenvectors of $M$. Thus, the basic goal of this Chapter is to find the eigenvectors and eigenvalues of the one-turn map.

From one perspective, this is a solved, relatively trivial, problem. Given a numerical representation of $M$, it is straight-forward to find the eigenvalues and eigenvectors. It is, however, an analytical representation that we seek. In particular, we are interested in the following quite common situation. A storage ring is designed to be uncoupled. In Chapter 2 we found the eigenvectors of an uncoupled ring, expressing them in terms of lattice parameters. A real ring, however will have small perturbations to this uncoupled ring. We would like to find analytical expressions for the 
eigenvalues and eigenvectors in the presence of small perturbations. We will be especially interested in the case of a linear resonance where two of the unperturbed eigenvalues are close in value. In this case, a small perturbation can cause a large effect.

A complication arises, however, when doing perturbation theory directly on $M_{\text {uncoupled }}$ near a resonance. At the degeneracy, the eigenvectors are also degenerate, meaning that linear combinations of eigenvectors are still eigenvectors. One must add a perturbation to break the degeneracy and pick out specific eigenvectors. ${ }^{1}$ The degeneracy is broken by two relevant small quantities. First, there is the distance in tune space from resonance, and second, there is the coupling. Interesting perturbative effects occur when these two quantities are of similar size. The correct lowest order eigenvectors are not necessarily those of $M_{\text {uncoupled }}$. We will thus consider $M_{\text {uncoupled }}$ to be $M_{0}+M_{1 \mu}$ where $M_{0}$ is the map exactly on resonance. Instead of doing perturbation theory on $M_{\text {uncoupled, }}$, we will do it on $M_{0}$. If we turn off the coupling, we will of course expect to get the correct eigenvalues and eigenvectors for $M_{\text {uncoupled }}$.

The resonances we consider occur when two of the eigenvalues are equal. We will specialize to a 4 -D subspace in which we can capture this dynamics. Extension to the full 6-D phase space is not difficult, but does not add much new dynamics while it would make the formalism more cumbersome. We take our 4-D space to be $x-z$ phase space, but it also applies to $x-y$ and $y-z$.

There are many ways to define such an on-resonance $M_{0}$. We choose to do so in such a way that the eigenvectors remain to be given by Eq.(2.40). This can be accomplished by choosing

$$
M_{0}=\left(\begin{array}{cc}
M_{x 0} & 0 \\
0 & M_{z 0}
\end{array}\right),
$$

where

$$
M_{x 0}=e^{\mu_{x 0} J_{x}}, \quad M_{z 0}=e^{\mu_{z 0} J_{z}},
$$

where $\mu_{x 0}$ and $\mu_{z 0}$ are their degenerate (exactly on resonance) values and $J_{x}$ and $J_{z}$ are defined as before, i.e. by (2.30). In particular, $\alpha_{x, z}, \beta_{x, z}$, and $\gamma_{x, z}$ are the

\footnotetext{
${ }^{1}$ For more on this point, see a discussion of degenerate perturbation theory in quantum mechanics, such as in [67], pp. 227-231, as well as our derivations in the next section and in Appendix B.
} 
uncoupled lattice functions derived from $M_{\text {uncoupled }}$. Let us write ${ }^{2} \mu_{x}=\mu_{x 0}+\delta \mu_{x} / 2$ and $\mu_{z}=\mu_{z 0}+\delta \mu_{z} / 2$, then the perturbation to $M_{0}$ is given to lowest order as

$$
\begin{aligned}
M_{1 \mu} & =\left(\begin{array}{cc}
\frac{\delta \mu_{x}}{2} J_{x} M_{x 0} & 0 \\
0 & \frac{\delta \mu_{z}}{2} J_{z} M_{z 0}
\end{array}\right) \\
& =\left(\begin{array}{cc}
\frac{\delta \mu_{x}}{2} J_{x} & 0 \\
0 & \frac{\delta \mu_{z}}{2} J_{z}
\end{array}\right) M_{0} .
\end{aligned}
$$

For example, in the case of the sum resonance, $\mu_{x}+\mu_{z}=\delta \mu$ where $\delta \mu$ is small (recall the redefinition Eq. (2.31). We then write $\mu_{x 0}=\mu, \mu_{z 0}=-\mu$, and $\delta \mu_{x}=\delta \mu_{z}=\delta \mu$. For the difference resonance, $\mu_{x}-\mu_{z}=\delta \mu$, and we have $\mu_{x 0}=\mu_{z 0}=\mu$ and $\delta \mu_{x}=$ $-\delta \mu_{z}=\delta \mu$. For the integer resonance in $\mu_{x}$, we have $\mu_{x 0}=\mu_{-x 0}=0$ and $\delta \mu_{x}=2 \mu_{x}$. In this case $\mu_{z 0}$ can be anything; although in practice, the synchrotron tune will typically be small. Finally, for a half-integer resonance in $\mu_{x}$, we have $\mu_{x 0}=\pi$, and $\delta \mu_{x}=2\left(\mu_{x}-\pi\right)$. Along with the corresponding $\mu_{z}$ integer and half-integer resonances, these six cases covers all the single linear resonances.

The matrix $M_{1 \mu}$ specifies the deviation of the uncoupled map from being exactly on resonance. In addition to the perturbation $M_{1 \mu}$, we still have to add the perturbation coming from the coupling. We designate its contribution to the one-turn map as $M_{1 \xi}$. This could come from an error in the lattice, or from an added coupling element (such as a crab cavity) somewhere in the ring, or in the case of dispersion at an RF cavity, which can be considered to be intrinsically coupling (not due to errors). We show how to find $M_{1 \xi}$ for a dispersive RF cavity and a crab cavity in a later section. We write

$$
M=M_{0}+M_{1}, \quad M_{1}=M_{1 \mu}+M_{1 \xi}
$$

As mentioned, $M_{1 \mu}$ does not change the eigenvectors of $M_{0} ; M_{1 \xi}$ will, however. To be more explicit about the perturbation $M_{1 \xi}$, suppose that at position $s_{j}$ we insert a perturbation $1+P\left(s_{j}\right)$. Let there be $n$ such perturbations, arranged such that $0<s_{1}<s_{2}<\ldots<s_{n}<C$. We have defined the observation position to be $s=0$.

\footnotetext{
${ }^{2}$ The factor of $1 / 2$ is for later convenience - it allows for the interpretation of $\delta \mu_{x, z}$ as the splitting between the uncoupled nearly degenerate phase advances.
} 
The new one-turn map at the observation position is

$$
M=T_{s_{n} \rightarrow C}\left[1+P\left(s_{n}\right)\right] \ldots T_{s_{1} \rightarrow s_{2}}\left[1+P\left(s_{1}\right)\right] T_{0 \rightarrow s_{1}}
$$

Keeping lowest order in the perturbations, we find

$$
M_{1 \xi}=\left(\sum_{m=1}^{n} T_{s_{m} \rightarrow C} P\left(s_{m}\right) T_{0 \rightarrow s_{m}}^{-1}\right) M_{\text {uncoupled }}(0) \text {. }
$$

\subsection{Perturbation to Eigenvalues and Eigenvectors}

Because $M_{0}$ is degenerate, we use degenerate perturbation theory to find the perturbed eigenvectors and eigenvalues. We here give a brief derivation of the results we will need for most of what follows. The discussion is carried out in greater generality and detail in Appendix B.

Consider specific values of $j$ and $k$ such that a degeneracy occurs with $\mu_{j 0}=$ $\mu_{k 0}=\mu_{0}$. Then $v_{j 0}$ and $v_{k 0}$ form a degenerate subspace of $M_{0}$; that is, any linear combinations of $v_{j 0}$ and $v_{k 0}$ are still degenerate eigenvectors of $M_{0}$. The perturbation will pick out a particular linear combination in addition to perturbing it, thus breaking the degeneracy. We write this as

$$
\begin{aligned}
& v_{j}=\tilde{v}_{j 0}+\tilde{v}_{j 1}+\ldots, \\
& v_{k}=\tilde{v}_{k 0}+\tilde{v}_{k 1}+\ldots,
\end{aligned}
$$

where $\tilde{v}_{j 0}$ and $\tilde{v}_{k 0}$ are the 0th order linear combinations:

$$
\begin{aligned}
& \tilde{v}_{j 0}=c_{j 0}^{j} v_{j 0}+c_{k 0}^{j} v_{k 0}, \\
& \tilde{v}_{k 0}=c_{j 0}^{k} v_{j 0}+c_{k 0}^{k} v_{k 0},
\end{aligned}
$$

where $v_{j 0}$ and $v_{k 0}$ are the eigenvectors of $M_{0}$ with eigenvalues $\lambda_{j 0}$ and $\lambda_{k 0}$, and are 
identified with two of the four candidates $v_{x}, v_{-x}, v_{z}$, and $v_{-z}$ from Eq.(2.40) as the case may be.

We then expand the eigenvalues up to 1st order,

$$
\begin{aligned}
\lambda_{j} & =\lambda_{j 0}+\lambda_{j 1}+\ldots, \\
\lambda_{k} & =\lambda_{k 0}+\lambda_{k 1}+\ldots,
\end{aligned}
$$

The eigenvalue equations up to 1 st order for $v_{j}$ and $v_{k}$ say

$$
\begin{aligned}
\left(M_{0}+M_{1}\right)\left(\tilde{v}_{j 0}+\tilde{v}_{j 1}\right) & =\left(\lambda_{j 0}+\lambda_{j 1}\right)\left(\tilde{v}_{j 0}+\tilde{v}_{j 1}\right), \\
\left(M_{0}+M_{1}\right)\left(\tilde{v}_{k 0}+\tilde{v}_{k 1}\right) & =\left(\lambda_{k 0}+\lambda_{k 1}\right)\left(\tilde{v}_{k 0}+\tilde{v}_{k 1}\right) .
\end{aligned}
$$

We now multiply by $v^{j 0}$ and $v^{k 0}$ on the left to each of these equations. We again keep up to first order. After using the 0th order eigenvalue equation, and using the expansion in Eq.(3.8), we find the eigenvalue equation

$$
\left(\begin{array}{ll}
\mathcal{M}_{j j} & \mathcal{M}_{j k} \\
\mathcal{M}_{k j} & \mathcal{M}_{k k}
\end{array}\right)\left(\begin{array}{c}
c_{j 0}^{j, k} \\
c_{k 0}^{j, k}
\end{array}\right)=\lambda_{j, k 1}\left(\begin{array}{c}
c_{j 0}^{j, k} \\
c_{k 0}^{j, k}
\end{array}\right),
$$

where we have defined

$$
\mathcal{M}_{m n}=v^{m 0} M_{1} v_{n 0}
$$

Thus, we see that in order to find the coefficients for the coupled eigenvectors, we need to find the eigenvectors of this perturbation matrix $\mathcal{M}$, whereas to find the perturbation to the eigenvalues, we find its eigenvalues.

The matrix elements of $\mathcal{M}$ are mutually interrelated because $M_{1}$ is not arbitrary but must be such that $M$ is symplectic. We write $M=M_{0}+M_{1}$ and apply the 
symplectic condition (2.7). Given that $M_{0}$ is symplectic, we find, to lowest order, ${ }^{3}$

$$
M_{0}^{T}\left(J M_{1}\right)=\left(J M_{1}\right)^{T} M_{0},
$$

which says that $M_{0}^{T} J M_{1}$ is a symmetric matrix. Taking a transpose and conjugate of Eq. (3.12), and using this equation and the definition (2.11), we can now prove the following relationships among the matrix elements $\mathcal{M}_{m n}(m, n= \pm 1, \pm 2)$ :

$$
\begin{aligned}
\mathcal{M}_{m n} & =-\operatorname{sgn}(m) \operatorname{sgn}(n) \lambda_{m 0} \lambda_{n 0} \mathcal{M}_{n m}^{*}, \\
\mathcal{M}_{m n} & =\mathcal{M}_{-m-n}^{*} .
\end{aligned}
$$

For later convenience, we would also like to define some additional related quantities. As we saw in Eqs.(3.3) and (3.6), it is often convenient to write $M_{1}$ in the form

$$
M_{1}=P M_{0} .
$$

We then define a "coupling parameter"

$$
r_{m n}=v^{m 0} P v_{n 0},
$$

from which follows that

$$
r_{m n}=e^{-i \mu_{n 0}} \mathcal{M}_{m n}
$$

In terms of the $r_{m n}$, the relations (3.14) and (3.15) state

$$
\begin{aligned}
& r_{m n}=-\operatorname{sgn}(m) \operatorname{sgn}(n) r_{n m}^{*}, \\
& r_{m n}=r_{-m-n}^{*} .
\end{aligned}
$$

We now solve the eigenvalue equation (3.11). In terms of the $r_{m n}$, the eigenvalues

\footnotetext{
${ }^{3}$ In the case that $M_{1}$ is proportional to some parameter and that $M$ is symplectic for some continuous range of that parameter, then Eq.(3.13) will be true exactly, not just to lowest order, which one sees by writing out the symplectic condition as a Taylor series in this parameter and setting terms of the same order equal to each other. This situation applies to the cases considered in this paper. For the RF cavity, this parameter is $r$, and for the crab cavity it is $\xi_{c}$, see Eqs.(3.116) and (3.137).
} 
are

$$
\begin{aligned}
& \lambda_{j 1}=\frac{e^{i \mu_{0}}}{2}\left[\left(r_{j j}+r_{k k}\right)+\left(r_{j j}-r_{k k}\right) \sqrt{1+\frac{4 r_{j k} r_{k j}}{\left(r_{j j}-r_{k k}\right)^{2}}}\right], \\
& \lambda_{k 1}=\frac{e^{i \mu_{0}}}{2}\left[\left(r_{j j}+r_{k k}\right)-\left(r_{j j}-r_{k k}\right) \sqrt{1+\frac{4 r_{j k} r_{k j}}{\left(r_{j j}-r_{k k}\right)^{2}}}\right] .
\end{aligned}
$$

From the perturbations to the eigenvalues, we can find the perturbation to the phase advances. To this end, we write

$$
\begin{aligned}
& \lambda_{j}=\lambda_{j 0}+\lambda_{j 1}=e^{i\left(\mu_{0}+\mu_{j 1}\right)} \approx e^{i \mu_{0}}\left(1+i \mu_{j 1}\right), \\
& \lambda_{k}=\lambda_{k 0}+\lambda_{k 1}=e^{i\left(\mu_{0}+\mu_{k 1}\right)} \approx e^{i \mu_{0}}\left(1+i \mu_{k 1}\right) .
\end{aligned}
$$

from which we see that the first order perturbation to the phase advances are given by

$$
\begin{aligned}
& \mu_{j 1}=-i e^{-i \mu_{0}} \lambda_{j 1} \\
& \mu_{k 1}=-i e^{-i \mu_{0}} \lambda_{k 1}
\end{aligned}
$$

Let us decompose the shift in the phase advances into an average shift and difference from that average. i.e. we write

$$
\begin{aligned}
& \mu_{j 1}=\bar{\mu}+\frac{\delta \mu_{1}}{2} \\
& \mu_{k 1}=\bar{\mu}-\frac{\delta \mu_{1}}{2}
\end{aligned}
$$

which implies

$$
\begin{aligned}
\bar{\mu} & =\frac{1}{2}\left(\mu_{j 1}+\mu_{k 1}\right), \\
\delta \mu_{1} & =\mu_{j 1}-\mu_{k 1} .
\end{aligned}
$$


Using (3.25), (3.23) and (3.21), we find

$$
\begin{aligned}
\bar{\mu} & =-i\left(r_{j j}+r_{k k}\right), \\
\delta \mu_{1} & =-\frac{i}{2}\left(r_{j j}-r_{k k}\right) \sqrt{1+\frac{4 r_{j k} r_{k j}}{\left(r_{j j}-r_{k k}\right)^{2}}} .
\end{aligned}
$$

Now, using Eq.(3.19), $r_{j j}$ and $r_{k k}$ are purely imaginary. We can then define the quantity

$$
\Delta \mu=-i\left(r_{j j}-r_{k k}\right)
$$

and we know that it will be real. In fact, it is useful to focus on this term a bit more for the moment. Recall that $M_{1}=M_{1 \mu}+M_{1 \xi}$. We correspondingly write $r_{m n}=r_{m n \mu}+r_{m n \xi}$ and $\Delta \mu=\Delta \mu_{\mu}+\Delta \mu_{\xi}$ for the two corresponding parts of $r_{m n}$ and $\Delta \mu$. The form of $M_{1 \mu}$ was given in Eq.(3.3). Note that $M_{0}$ factored out so that the perturbation coming from the difference from resonance, $P_{\mu}$ is

$$
P_{\mu}=\left(\begin{array}{cc}
\delta \mu_{x} J_{x} & 0 \\
0 & \delta \mu_{z} J_{z}
\end{array}\right)
$$

Now, one can easily show that (ignoring the 0's in the $z$ and $\delta$ components) $v_{x}$ is an eigenvector of $J_{x}$ with eigenvalue $i$, and $v_{-x}$ with eigenvalue $-i$. Likewise for $v_{z}$ and $v_{-z}$. Using this, we immediately get that $r_{11 \mu}=-r_{-1-1 \mu}=i \delta \mu_{x}$ and $r_{22 \mu}=r_{-2-2 \mu}=i \delta \mu_{z}$. We have given the expressions for $\delta \mu_{x, z}$ in the paragraph following Eq.(3.3). The result is that $\Delta \mu_{\mu}$ is $\delta \mu=\mu_{x}-\mu_{z}$ for the difference resonance, $\delta \mu=\mu_{x}+\mu_{z}$ for the sum resonance, and $2 \mu_{x}$ or $2\left(\mu_{x}-\pi\right)$ for an $x$ integer or half integer resonance, respectively and likewise for $z$. Then we can write

$$
\Delta \mu=\delta \mu-i\left(r_{j j \xi}-r_{k k \xi}\right)
$$

$\Delta \mu$ will be one of the main parameters we need to compute in any given example. We refer to it as the "splitting parameter". The additional term involving the perturbation gives the direct change to the tune split (times $2 \pi$ ) assuming $r_{j k \xi}=0$. 
Next, using Eq.(3.19), we find

$$
r_{j k} r_{k j}=-\operatorname{sgn}(j) \operatorname{sgn}(k)\left|r_{j k}\right|^{2} .
$$

If we then define

$$
\xi=2\left|r_{j k}\right|,
$$

Eq.(3.27) becomes

$$
\delta \mu_{1}=\frac{\Delta \mu}{2} \sqrt{1+\operatorname{sgn}(j) \operatorname{sgn}(k) \frac{\xi^{2}}{\Delta \mu^{2}}} .
$$

In the case where one mode is positive and the other negative, we see that $\delta \mu_{1}$ becomes imaginary and hence we have an instability for

$$
\xi>|\Delta \mu|
$$

whereas if both modes are positive or both negative, there is no instability. It is here that we see why the difference resonance is always stable, whereas the sum, integer and half-integer resonances can have instabilities. In the case of the difference resonance, the positive $x$ mode is degenerate with the positive $z$ mode and likewise for the negative modes. For the sum resonance, the positive $x$ mode is degenerate with the negative $z$ mode and the negative $x$ mode degenerate with the positive $z$ mode. We can also understand the instability of the integer and half-integer resonances. In the case of the $x$ integer and half-integer resonances, the positive $x$ mode is degenerate with the negative $x$ mode, whereas for the $z$ integer and half-integer resonances, the positive and negative $z$ modes are degenerate. So, the difference resonance has degenerate modes of the same sign and hence stability, while the sum, integer and halfinteger resonances have degenerate modes of opposite signs and hence the possibility of instability.

Next, consider the eigenvectors of $\mathcal{M}$. These can be written as

$$
\left(\begin{array}{c}
c_{j 0}^{j} \\
c_{k 0}^{j}
\end{array}\right)=\left(\begin{array}{c}
\frac{r_{j j}-r_{k k}}{2 r_{k j}}\left[1+\sqrt{1+\frac{4 r_{j k} r_{k j}}{\left(r_{j j}-r_{k k}\right)^{2}}}\right. \\
1
\end{array}\right),
$$




$$
\left(\begin{array}{c}
c_{j 0}^{k} \\
c_{k 0}^{k}
\end{array}\right)=\left(\begin{array}{c}
\frac{r_{j j}-r_{k k}}{2 r_{k j}}\left[1-\sqrt{1+\frac{4 r_{j k} r_{k j}}{\left(r_{j j}-r_{k k}\right)^{2}}}\right. \\
1
\end{array}\right)
$$

These eigenvectors have yet to be normalized. All of the quantities here fit nicely into the definitions we have already made except for the $r_{k j}$ in the denominator. We know that it has an absolute value of $\frac{\xi}{2}$, but it also has an additional phase. Let us thus define a phase $\phi$ by

$$
r_{j k}=\frac{\xi}{2} e^{i \phi}
$$

or

$$
\phi=\arg \left(r_{j k}\right)
$$

where $\arg ()$ means taking the angle in the complex plane with $\phi \in[-\pi, \pi]$. We use (3.19) to relate $r_{j k}$ to $r_{k j}$, noting that we get an additional minus sign when $j$ and $k$ have the same sign. The eigenvectors look different depending on whether the modes have the same or different signs. When both modes have the same sign, after normalization, we can express the eigenvectors as

$$
\begin{aligned}
& \tilde{v}_{j 0}=\cos \frac{\theta}{2} v_{j 0}+i e^{-i \phi} \sin \frac{\theta}{2} v_{k 0}, \\
& \tilde{v}_{k 0}=i e^{i \phi} \sin \frac{\theta}{2} v_{j 0}+\cos \frac{\theta}{2} v_{k 0},
\end{aligned}
$$

where

$$
\tan \theta=\frac{\xi}{\Delta \mu}
$$

When the modes have opposite signs and supposing that $j$ is the positive mode, we find the normalized eigenvectors to be

$$
\begin{aligned}
\tilde{v}_{j 0} & =\cosh \frac{\theta}{2} v_{j 0}-i e^{-i \phi} \sinh \frac{\theta}{2} v_{k 0} \\
\tilde{v}_{k 0} & =i e^{i \phi} \sinh \frac{\theta}{2} v_{j 0}+\cosh \frac{\theta}{2} v_{k 0}
\end{aligned}
$$

where

$$
\tanh \theta=\frac{\xi}{\Delta \mu}
$$


In all cases, the quantity $\theta$ will be referred to as the "coupling angle". We have normalized these eigenvectors such that

$$
\tilde{v}^{j 0} \tilde{v}_{k 0}=\delta_{j k}
$$

The overall phases are chosen so that for $\theta=0, \tilde{v}_{j 0}=v_{j 0}$ and $\tilde{v}_{k 0}=v_{k 0}$. Note also that for this choice of overall phase, for the cases when $k=-j$, i.e. the integer or half integer resonance, the condition that $\tilde{v}_{j 0}=i \tilde{v}_{k 0}^{*}$ is satisfied.

\subsection{Cases of Resonances}

We now consider the specific cases for values of $j$ and $k$. We will first consider the integer and half integer resonances and then the sum and difference resonances. The integer and half integer resonances involve a single pair of degenerate eigenvalues: for the integer resonance, an eigenvalue pair approaches the positive real axis while for the half integer resonance, a pair approaches the negative real axis. In the cases of the sum and difference resonances, both eigenvalue pairs become degenerate.

\subsubsection{Integer/Half Integer Resonance}

The integer/half integer resonances are covered by the cases where $(j, k)$ equals $(1,-1)$ or $(2,-2)$. Let us consider the case $(j, k)=(2,-2)$. This is the case of an integer or half-integer resonance for $\mu_{z}$. For the integer resonance, we have $\mu_{z 0}=-\mu_{-z 0}=0$ and for the half-integer resonance, we have $\mu_{z 0}=-\mu_{-z 0}=\pi$. Here, the perturbation matrix is

$$
\left(\begin{array}{ll}
\mathcal{M}_{22} & \mathcal{M}_{2-2} \\
\mathcal{M}_{-22} & \mathcal{M}_{-2-2}
\end{array}\right) .
$$

The coupling parameter is given by

$$
\xi=2\left|r_{2-2}\right|
$$


The splitting parameter $\Delta \mu$ is given by

$$
\Delta \mu=2 \mu_{z}-2 i r_{22 \xi}
$$

for the integer resonance and

$$
\Delta \mu=2\left(\mu_{z}-\pi\right)-2 i r_{22 \xi}
$$

for the half integer resonance. The average shift in the phase advances of $\mu_{ \pm z}, \bar{\mu}$, is given by

$$
\bar{\mu}=-i\left(r_{22 \xi}+r_{-2-2 \xi}\right)=2 \operatorname{Re}\left(r_{22 \xi}\right)=0,
$$

where we have used (3.19) and (3.20). This result is expected due to the fact that $\mu_{2}=-\mu_{-2}$. In terms of these quantities, then, we can express the perturbed $x$ eigen-phase advance to first order as

$$
\mu_{2}=\frac{\Delta \mu}{2} \sqrt{1-\frac{\xi^{2}}{\Delta \mu^{2}}} .
$$

for the integer resonance and

$$
\mu_{2}=\pi+\frac{\Delta \mu}{2} \sqrt{1-\frac{\xi^{2}}{\Delta \mu^{2}}}
$$

for the half integer resonance. Note that these reduce to $\mu_{z}$ when the perturbation is turned off. The coupling angle is defined by

$$
\tanh \theta=\frac{\xi}{\Delta \mu}
$$

so that $\theta$ ranges from $-\infty$ to $\infty$ with the sign determined by the sign of $\Delta \mu$.

In terms of this angle, we can express the eigen-phase advance as

$$
\mu_{2}=\frac{\Delta \mu}{2} \operatorname{sech} \theta
$$


for the integer resonance, with $\Delta \mu$ given in (3.45), or

$$
\mu_{2}-\pi=\frac{\Delta \mu}{2} \operatorname{sech} \theta
$$

for the half integer resonance, with $\Delta \mu$ given in (3.46). Note that as $\theta$ gets large, the eigen-phase advance goes to 0 . At the instability, $\theta$ passes through infinity and $\mu_{2}$ becomes complex.

Finally, the relative phase for the eigenvectors is given by

$$
\phi=\arg \left(r_{2-2}\right)
$$

for both the integer and half-integer resonance. Eq. (3.40) then gives the eigenvectors in terms of these quantities as

$$
\begin{aligned}
\tilde{v}_{2} & =\cosh \frac{\theta}{2} v_{z}-i e^{-i \phi} \sinh \frac{\theta}{2} v_{-z} \\
\tilde{v}_{-2} & =i e^{i \phi} \sinh \frac{\theta}{2} v_{z}+\cosh \frac{\theta}{2} v_{-z}
\end{aligned}
$$

We can write these explicitly as

$$
\tilde{v}_{2}=\frac{1}{\sqrt{2}}\left(\begin{array}{c}
\sqrt{\beta_{z}}\left[\cosh \left(\frac{\theta}{2}\right)-e^{-i \phi} \sinh \left(\frac{\theta}{2}\right)\right] \\
\frac{1}{\beta_{z}}\left[\left(i-\alpha_{z}\right) \cosh \left(\frac{\theta}{2}\right)+\left(i+\alpha_{z}\right) e^{-i \phi} \sinh \left(\frac{\theta}{2}\right)\right] \\
0 \\
0
\end{array}\right)
$$

and $\tilde{v}_{-2}=-i \tilde{v}_{2}^{*}$. Note that only the $2-\mathrm{D} z$ phase space components of the eigenvectors get mixed together by the perturbation. Further, only this subspace of the perturbation matrix enters into the coupling angle and phase, as is evident in (3.43). Thus, this is a fully 2-D calculation. One might wonder what happened to the coupling, which is a 4-D phenomena. It turns out that we have in fact missed the coupling, and that to include it appropriately, we need to go to second order perturbation theory. We find that the eigenvectors are still given by (3.54), but now the expressions for the coupling angle and phase includes the full 4-D coupling perturbation matrix. We 
discuss this further in Section 3.2.4.

For the case of the $\mu_{x}$ integer and half-integer resonances, the perturbation matrix is

$$
\left(\begin{array}{cc}
\mathcal{M}_{11} & \mathcal{M}_{1-1} \\
\mathcal{M}_{-11} & \mathcal{M}_{-1-1}
\end{array}\right)
$$

and the preceding results are repeated with 2 and $z$ replaced by 1 and $x$.

\subsubsection{Sum Resonance}

Next we consider the sum resonance where we have the degeneracies $(j, k)=(1,-2)$ and $(j, k)=(-1,2)$. Note that both of these degeneracies must occur together because of symplectity of $M$. The perturbation matrices are

$$
\left(\begin{array}{ll}
\mathcal{M}_{11} & \mathcal{M}_{1-2} \\
\mathcal{M}_{-21} & \mathcal{M}_{-2-2}
\end{array}\right), \quad\left(\begin{array}{cc}
\mathcal{M}_{-1-1} & \mathcal{M}_{-12} \\
\mathcal{M}_{2-1} & \mathcal{M}_{22}
\end{array}\right) .
$$

We find a single coupling parameter

$$
\xi=2\left|r_{1-2}\right|
$$

$\Delta \mu$ is given by

$$
\Delta \mu=\mu_{x}+\mu_{z}-i\left(r_{11 \xi}-r_{-2-2 \xi}\right)
$$

The coupling angle is now defined by

$$
\tanh \theta=\frac{\xi}{\Delta \mu}
$$

Next, $\bar{\mu}$ is given by

$$
\bar{\mu}=-i\left(r_{11 \xi}+r_{-2-2 \xi}\right) .
$$

The eigen-phase advances are

$$
\begin{aligned}
\mu_{1} & =\mu+\bar{\mu}+\frac{\Delta \mu}{2} \operatorname{sech} \theta \\
\mu_{2}=-\mu_{-2} & =-\mu-\bar{\mu}+\frac{\Delta \mu}{2} \operatorname{sech} \theta
\end{aligned}
$$


The phase for the eigenvectors is given by

$$
\phi=\arg \left(r_{1-2}\right)
$$

The eigenvectors are then given in terms of these quantities as

$$
\begin{aligned}
\tilde{v}_{1} & =\cosh \frac{\theta}{2} v_{x}-i e^{-i \phi} \sinh \frac{\theta}{2} v_{-z} \\
\tilde{v}_{-2} & =i e^{i \phi} \sinh \frac{\theta}{2} v_{x}+\cosh \frac{\theta}{2} v_{-z}
\end{aligned}
$$

The other two eigenvectors are given by $v_{-1}=-i v_{1}^{*}$ and $v_{2}=-i v_{-2}^{*}$.

\subsubsection{Difference Resonance}

For the difference resonance, we have the degeneracies $(1,2)$ and $(-1,-2)$. The perturbation matrices are

$$
\left(\begin{array}{ll}
\mathcal{M}_{11} & \mathcal{M}_{12} \\
\mathcal{M}_{21} & \mathcal{M}_{22}
\end{array}\right), \quad\left(\begin{array}{ll}
\mathcal{M}_{-1-1} & \mathcal{M}_{-1-2} \\
\mathcal{M}_{-2-1} & \mathcal{M}_{-2-2}
\end{array}\right) .
$$

We find a single coupling parameter

$$
\xi=2\left|r_{12}\right|
$$

$\Delta \mu$ is given by

$$
\Delta \mu=\mu_{x}-\mu_{z}-i\left(r_{11}-r_{22}\right) .
$$

The coupling angle is then given by

$$
\tan \theta=\frac{\xi}{\Delta \mu}
$$


Here $\theta$ can range from $-\pi / 4$ to $\pi / 4$, again with $\Delta \mu$ determining the sign. The eigen-phase advances are

$$
\begin{aligned}
& \mu_{1}=\mu+\bar{\mu}+\frac{\Delta \mu}{2} \sqrt{1+\frac{\xi^{2}}{\Delta \mu^{2}}} \\
& \mu_{2}=\mu+\bar{\mu}-\frac{\Delta \mu}{2} \sqrt{1+\frac{\xi^{2}}{\Delta \mu^{2}}} .
\end{aligned}
$$

In terms of the coupling angle, we can express these as

$$
\begin{aligned}
& \mu_{1}=\mu+\bar{\mu}+\frac{\Delta \mu}{2} \sec \theta \\
& \mu_{2}=\mu+\bar{\mu}-\frac{\Delta \mu}{2} \sec \theta
\end{aligned}
$$

The phase for the eigenvectors is given by

$$
\phi=\arg \left(r_{12}\right) .
$$

We can now construct the eigenvectors from (3.38)

$$
\begin{aligned}
& \tilde{v}_{1}=\cos \frac{\theta}{2} v_{x}+i e^{-i \phi} \sin \frac{\theta}{2} v_{z} \\
& \tilde{v}_{2}=i e^{i \phi} \sin \frac{\theta}{2} v_{x}+\cos \frac{\theta}{2} v_{z} .
\end{aligned}
$$

The other two eigenvectors are $v_{-1}=-i v_{1}^{*}$ and $v_{-2}=-i v_{2}^{*}$.

The results for $\xi, \Delta \mu, \phi$, and $\bar{\mu}$ for each of these resonances are summarized in the first six rows of Table 3.1.

\subsubsection{Special Case of an Integer/Half-Integer Resonance Caused by Coupling}

We have seen that there is a subtlety related to integer and half-integer resonances. One can see that the "true $x-z$ coupling" from $r_{12}$ or $r_{1-2}$ did not enter into the results. In fact, the integer/half integer results we have derived follow from a strictly 2-D phase 


\begin{tabular}{|c|c|c|c|c|c|}
\hline reso. & condition & $\Delta \mu(\bmod 2 \pi)$ & $\xi$ & $\phi$ & $\bar{\mu}$ \\
\hline sum & $\mu_{x}+\mu_{z}=2 \pi n$ & $\mu_{x}+\mu_{z}-i\left(r_{11}-r_{-2-2}\right)$ & $2\left|r_{1-2}\right|$ & $\arg \left(r_{1-2}\right)$ & $-i\left(r_{11}+r_{-2-2}\right)$ \\
\hline diff. & $\mu_{x}-\mu_{z}=2 \pi n$ & $\mu_{x}-\mu_{z}-i\left(r_{11}-r_{22}\right)$ & $2\left|r_{12}\right|$ & $\arg \left(r_{12}\right)$ & $-i\left(r_{11}+r_{22}\right)$ \\
\hline int $(x)$ & $\mu_{x}=2 \pi n$ & $2 \mu_{x}-2 i r_{11}$ & $2\left|r_{1-1}\right|$ & $\arg \left(r_{1-1}\right)$ & 0 \\
\hline $\operatorname{int}(z)$ & $\mu_{z}=2 \pi n$ & $2 \mu_{z}-2 i r_{22}$ & $2\left|r_{2-2}\right|$ & $\arg \left(r_{2-2}\right)$ & 0 \\
\hline$\frac{1}{2}-\operatorname{int}(x)$ & $\mu_{x}=\pi(2 n+1)$ & $2\left(\mu_{x}-\pi\right)-2 i r_{11}$ & $2\left|r_{1-1}\right|$ & $\arg \left(r_{1-1}\right)$ & 0 \\
\hline$\frac{1}{2}-\operatorname{int}(z)$ & $\mu_{z}=\pi(2 n+1)$ & $2\left(\mu_{z}-\pi\right)-2 i r_{22}$ & $2\left|r_{2-2}\right|$ & $\arg \left(r_{2-2}\right)$ & 0 \\
\hline cp. int $(x)$ & $\mu_{x}=2 \pi n$ & $\begin{array}{c}2 \mu_{x}-2 i r_{11} \\
-\left(\left|r_{12}\right|^{2}+\left|r_{-12}\right|^{2}\right) \cot \left(\frac{\mu_{z}}{2}\right)\end{array}$ & $2\left|r_{1-1}+i r_{2-1} r_{12} \cot \left(\frac{\mu_{z}}{2}\right)\right|$ & $\arg \left(r_{1-1}+i r_{2-1} r_{12} \cot \left(\frac{\mu_{z}}{2}\right)\right)$ & 0 \\
\hline cp. int $(z)$ & $\mu_{z}=2 \pi n$ & $\begin{array}{c}2 \mu_{z}-2 i r_{22} \\
-\left(\left|r_{12}\right|^{2}+\left|r_{-12}\right|^{2}\right) \cot \left(\frac{\mu_{x}}{2}\right) \\
\end{array}$ & $2\left|r_{2-2}+i r_{1-2} r_{21} \cot \left(\frac{\mu_{x}}{2}\right)\right|$ & $\arg \left(r_{2-2}+i r_{1-2} r_{21} \cot \left(\frac{\mu_{x}}{2}\right)\right)$ & 0 \\
\hline cp. $\frac{1}{2}$-int $(x)$ & $\mu_{x}=\pi(2 n+1)$ & $\begin{array}{c}2\left(\mu_{x}-\pi\right)-2 i r_{11} \\
+\left(\left|r_{12}\right|^{2}+\left|r_{-12}\right|^{2}\right) \tan \left(\frac{\mu_{z}}{2}\right) \\
\end{array}$ & $2\left|r_{1-1}-i r_{2-1} r_{12} \tan \left(\frac{\mu_{z}}{2}\right)\right|$ & $\arg \left(r_{1-1}-i r_{1-2} r_{21} \tan \left(\frac{\mu_{z}}{2}\right)\right)$ & 0 \\
\hline cp. $\frac{1}{2}$-int $(z)$ & $\mu_{z}=\pi(2 n+1)$ & $\begin{array}{c}2\left(\mu_{z}-\pi\right)-2 i r_{22} \\
+\left(\left|r_{12}\right|^{2}+\left|r_{-12}\right|^{2}\right) \tan \left(\frac{\mu_{x}}{2}\right) \\
\end{array}$ & $2\left|r_{2-2}-i r_{1-2} r_{21} \tan \left(\frac{\mu_{x}}{2}\right)\right|$ & $\arg \left(r_{2-2}-i r_{1-2} r_{21} \tan \left(\frac{\mu_{x}}{2}\right)\right)$ & 0 \\
\hline
\end{tabular}

Table 3.1: Linear resonances of a synchrobetatron coupled storage ring. For each of the linear resonances, we give general expressions for the quantities $\Delta \mu, \xi, \phi$, and $\bar{\mu}$. The quantities $r_{j k}$ here are $r_{j k \xi}$, i.e. the part of $r_{j k}$ coming from the coupling perturbation $P_{\xi}$. We have left off the subscript $\xi$ for convenience. The matrix elements $r_{j k \mu}$ coming from the difference from resonance are included explicitly in the $\Delta \mu$ and involved $\mu_{x}$ and $\mu_{z}$. The resonances with the abbreviation "cp." refer to the full 2 nd order calculation for the coupling-induced integer and half integer resonances (see Appendix D).

space analysis, reproducing results already contained in [22]. However, we are also interested in the situation where the perturbation is strictly a coupling perturbation, i.e., $M_{1 \xi}$ only has elements in the two off diagonal blocks. This will be the case for the perturbation due to a crab cavity when there is no dispersion at the cavity. We can then ask whether this coupling perturbation can cause an integer or halfinteger resonance if it is strong enough. Clearly our first order perturbation theory is insufficient to answer this question, and we thus consider 2nd order degenerate perturbation theory. We do this in Appendix B, providing an alternative derivation and further details in Appendix D. The result is that we can use the same results as above for the integer or half-integer resonance, except that we use as the perturbation matrix (consider the case when the resonance occurs in the $z$-dimension, i.e. $\mu_{z}$ is 
close to 0 or $\pi$ )

$$
\left(\begin{array}{cc}
\frac{\mathcal{M}_{12} \mathcal{M}_{21}}{\lambda_{20}-\lambda_{10}} & \frac{\mathcal{M}_{1-2} \mathcal{M}_{21}}{\lambda_{20}-\lambda_{10}} \\
+\frac{\mathcal{M}_{-12} \mathcal{M}_{2-1}}{\lambda_{20}-\lambda_{-10}} & +\frac{\mathcal{M}_{-1-2} \mathcal{M}_{2-1}}{\lambda_{20}-\lambda_{-10}} \\
+\mathcal{M}_{22} & +\mathcal{M}_{2-2} \\
& \\
\frac{\mathcal{M}_{12} \mathcal{M}_{-21}}{\lambda_{20}-\lambda_{10}} & \frac{\mathcal{M}_{1-2} \mathcal{M}_{-21}}{\lambda_{20}-\lambda_{10}} \\
+\frac{\mathcal{M}_{-12} \mathcal{M}_{-2-1}}{\lambda_{20}-\lambda_{-10}} & +\frac{\mathcal{M}_{-1-2} \mathcal{M}_{-2-1}}{\lambda_{20}-\lambda_{-10}} \\
+\mathcal{M}_{-22} & +\mathcal{M}_{-2-2}
\end{array}\right)
$$

We note that we have here a mixture of first and second order quantities. Neglecting the second order quantities reduces this to Eq.(3.56).

For the case of the integer $z$ resonance, this matrix simplifies to

$$
\left(\begin{array}{cc}
\frac{\left|r_{12}\right|^{2}}{1-e^{-i \mu_{x}}}-\frac{\left|r_{-12}\right|^{2}}{1-e^{i \mu_{x}}}+r_{22} & i r_{21} r_{1-2} \cot \left(\frac{\mu_{x}}{2}\right)+r_{2-2} \\
-i r_{21}^{*} r_{1-2}^{*} \cot \left(\frac{\mu_{x}}{2}\right)+r_{-22} & \frac{\left|r_{12}\right|^{2}}{1-e^{i \mu_{x}}}-\frac{\left|r_{-12}\right|^{2}}{1-e^{-i \mu_{x}}}+r_{-2-2}
\end{array}\right)
$$

where the $r_{j k}$ here are really $r_{j k \xi}$, the matrix elements due to just the coupling perturbation. The perturbed eigenvalues and eigenvectors are now given by the eigenvalues and eigenvectors of this matrix. We can again express them in terms of a coupling parameter, splitting parameter and phase. The coupling parameter and phase are defined by

$$
\xi e^{i \phi}=\frac{1}{2}\left[r_{2-2}+i r_{21} r_{1-2} \cot \left(\frac{\mu_{x}}{2}\right)\right]
$$

which reduce to (3.44) and (3.53) if we ignore the second order term.

The splitting parameter is given by

$$
\Delta \mu=2 \mu_{z}-2 i r_{22 \xi}-\left(\left|r_{12}\right|^{2}+\left|r_{-12}\right|^{2}\right) \cot \left(\frac{\mu_{x}}{2}\right)
$$

The results for the other integer and half integer resonance are summarized in the last four rows of Table 3.1. In terms of $\xi$ and $\Delta \mu$, we can again define a coupling angle

$$
\tanh \theta=\frac{\xi}{\Delta \mu}
$$

The eigen-phase advance is now given by (3.51). It is useful, however, to write 
out the expression for $\mu_{2}$ more explicitly. Let us consider the case in which the perturbation is purely a coupling perturbation $\left(r_{22 \xi}=r_{2-2 \xi}=0\right)$ and in which $\left|r_{12}\right|=\left|r_{1-2}\right|^{4}$. We then find

$$
\mu_{2}^{2}=\mu_{z}^{2}-\frac{1}{2} \mu_{z} \xi_{ \pm}^{2} \cot \left(\frac{\mu_{x}}{2}\right)
$$

where $\xi_{ \pm}=2\left|r_{12}\right|=2\left|r_{1-2}\right|$ is the coupling parameter for the sum or difference resonances, which will be equal based on our assumptions. We can now see that $\mu_{2}$ becomes unstable when

$$
\frac{1}{2} \xi_{ \pm}^{2} \cot \left(\frac{\mu_{x}}{2}\right)>\mu_{z}
$$

Applying a similar analysis, and under the same conditions, we find an instability near the half integer $z$ resonance when

$$
\frac{1}{2} \xi_{ \pm}^{2} \tan \left(\frac{\mu_{x}}{2}\right)<\pi-\mu_{z}
$$

The results for the $x$ integer and half integer resonances can be found by interchange of $x$ and $z$. Instabilities occur when

$$
\frac{1}{2} \xi_{ \pm}^{2} \cot \left(\frac{\mu_{z}}{2}\right)>\mu_{x}
$$

or

$$
\frac{1}{2} \xi_{ \pm}^{2} \tan \left(\frac{\mu_{z}}{2}\right)<\pi-\mu_{x}
$$

We now see that the 2nd order perturbation theory has yielded new instability conditions. The general expressions for $\mu_{1}$ and $\mu_{2}$ are given in Eqs.(D.23) and (D.24), but much of the basic physics can be seen by examining these results. Note how the coupling parameters for the sum or difference resonance enter into all of these expressions along with the value of the other phase advance not going unstable. Since $\mu_{z}$ is negative (above transition), we see that for the $z$ integer resonance, there can only be an instability when $\nu_{x}>\frac{1}{2}$. For the crab cavity, this is indeed an important resonance to consider because $\nu_{x}>\frac{1}{2}$ is a typical operation condition and for realistic

\footnotetext{
${ }^{4}$ See Appendix $\mathrm{C}$ for more on this condition.
} 
parameters, a storage ring can approach the instability region. For dispersion at an $\mathrm{RF}$ cavity, we will find that because of the form of $\xi_{ \pm}$(in particular, its dependence on the synchrotron tune), (3.79) is never satisfied and such an integer resonance never drives an instability.

\subsection{Evolution of Eigenvectors Around Ring}

We have given expressions for the eigenvectors near each of the resonances, computed at the position of the perturbation. In order to find global quantities like equilibrium invariants, we will need to know how the eigenvectors evolve around the ring. We can determine this using Eq.(2.51).

Let us consider the integer or half-integer resonance. Take mode 2 for example. We have seen that we can write the perturbed eigenvector in the form

$$
v_{2}\left(s_{1}\right)=\cosh \left(\frac{\theta}{2}\right) v_{z}\left(s_{1}\right)-i e^{-i \phi} \sinh \left(\frac{\theta}{2}\right) v_{-z}\left(s_{1}\right)
$$

Applying $T_{12 \beta}$ to this vector we find

$$
v_{2}\left(s_{2}\right)=e^{i \psi_{z 12}} \cosh \left(\frac{\theta}{2}\right) v_{z}\left(s_{2}\right)-i e^{-i\left(\phi+\psi_{z 12}\right)} \sinh \left(\frac{\theta}{2}\right) v_{-z}\left(s_{2}\right) .
$$

We have used the fact that $\psi_{-z 12}=-\psi_{z 12}$. The overall phase does not enter into any physical quantities, so we can multiply by $e^{-i \psi_{z 12}}$ and we find that the new eigenvector is that of the old, but with the lattice functions advanced to the new values and the phase $\phi\left(s_{2}\right)$ related to $\phi\left(s_{1}\right)$ by

$$
\phi\left(s_{2}\right)=\phi\left(s_{1}\right)+2 \psi_{z 12} .
$$

Likewise, for the $x$ integer/half integer resonance, the phase in the definition of $v_{1}$ is

$$
\phi\left(s_{2}\right)=\phi\left(s_{1}\right)+2 \psi_{x 12} .
$$


For the difference resonance, we apply the same argument and find

$$
\phi\left(s_{2}\right)=\phi\left(s_{1}\right)+\psi_{x 12}-\psi_{z 12}
$$

For the sum resonance, we find

$$
\phi\left(s_{2}\right)=\phi\left(s_{1}\right)+\psi_{x 12}+\psi_{z 12} .
$$

The phase advances $\psi_{x 12}$ and $\psi_{z 12}$ are given in Eqs.(2.45) and (2.49).

In the following section we will use these near resonance eigenvectors we have derived to construct the local damping and diffusion coefficients which are then integrated around the ring. We have computed the eigenvectors at an arbitrary position $s_{2}$ by first computing them at the position of the perturbation $s_{1}$ and then transforming them to $s_{2}$ by applying $T_{12 \beta}$. For consistency, we would like to see that we get the same result if we do the perturbation theory directly on the one-turn map at $s_{2}$. Writing $M=(1+P) M_{0}$ and transforming from position 1 to position 2 we find

$$
\begin{aligned}
\left(1+P\left(s_{2}\right)\right) M_{0}\left(s_{2}\right) & =M\left(s_{2}\right) \\
& =T_{12 \beta} M\left(s_{1}\right) T_{12 \beta}^{-1} \\
& =\left(1+T_{12 \beta} P\left(s_{1}\right) T_{12 \beta}^{-1}\right) M_{0}\left(s_{2}\right)
\end{aligned}
$$

So that

$$
P\left(s_{2}\right)=T_{12 \beta} P\left(s_{1}\right) T_{12 \beta}^{-1}
$$

Now, applying this equation along with (2.51) to the definition of $r_{m n}$, (3.17), and using the symplectic property of $T_{12 \beta}$, we find that $r_{m n}$ transforms as

$$
r_{m n}\left(s_{2}\right)=e^{i\left(\psi_{n 12}-\psi_{m 12}\right)} r_{m n}\left(s_{1}\right)
$$

i.e. they are invariant except for an overall phase change. From (3.91) we can see that $r_{j j}\left(s_{2}\right)=r_{j j}\left(s_{1}\right)$ and so all of the $\Delta \mu$ are global quantities. We can also show that the quantities $\xi$ are global quantities. For the sum, difference and 1st order integer and half-integer resonances, its obvious because of the absolute value. For the 
coupling caused integer and half-integer and half integer resonance, one must show that the two terms transform with the same phase. For example, using (3.91) several times on $\xi$ for the $x$ integer resonance (found in line 7 of table 3.1), we can show

$$
\begin{aligned}
\xi\left(s_{2}\right) & =\left|e^{-2 i \psi_{x 12}}\right| \xi\left(s_{1}\right) \\
& =\xi\left(s_{1}\right)
\end{aligned}
$$

and likewise for all the other $\xi$ 's. Thus, for each resonance, $\xi$ and $\Delta \mu$ are global quantities, independent of where they are calculated, and hence so is the coupling angle $\theta$. We can also use Eq. (3.91) to show that the expressions derived for $\phi(s)$ give the same results whether one first computes $\phi\left(s_{1}\right)$ and then uses $T_{12 \beta}$ to advance the eigenvectors to get $\phi\left(s_{2}\right)$, as we have done above, or if one first advances the $r_{m n}$ to $s_{2}$ and then computes the phase. The argument for the coupling caused integer and half-integer resonances is the same as that used in (3.92). Finally, we note that near each resonance, the net change in $\phi$ is a multiple of $2 \pi$ to lowest order, and thus, modulo $2 \pi, \phi$ is a well-defined, periodic function in the ring.

To summarize, the coupling angle $\theta$ is a global quantity independent of position in the ring, whereas $\phi$ is a phase that changes locally, but is periodic around the ring, modulo $2 \pi$. The formulas for $\phi$ given in Table I refer to the value at the position of the perturbation. We will use the eigenvectors to find the local invariants, which will depend on $\phi$ through $\cos \phi$ and $\sin \phi$. To advance these quantities to another position, $\phi$ must be advanced using Eqs. (3.85)-(3.88).

\subsection{Invariants}

With the addition of coupling near a linear resonance, the invariants $G_{x}$ and $G_{z}$ become perturbed to $G_{1}$ and $G_{2}$. We compute these approximately by using the lowest order perturbed eigenvectors $\tilde{v}_{j 0}$ derived earlier. 


\subsubsection{Sum/Difference resonance}

First consider the sum [Eqs.(3.40), (3.41), (3.58), and (3.59)] and difference resonances [Eqs.(3.38), (3.39), (3.66), and (3.67)]. We find

$$
\begin{aligned}
G_{1} & =\cosh ^{2} \frac{\theta}{2} G_{x}+\sinh ^{2} \frac{\theta}{2} G_{z}+\sinh \theta G_{c}^{+}, \\
G_{2} & =\sinh ^{2} \frac{\theta}{2} G_{x}+\cosh ^{2} \frac{\theta}{2} G_{z}+\sinh \theta G_{c}^{+},
\end{aligned}
$$

for the sum resonance, and

$$
\begin{aligned}
G_{1} & =\cos ^{2} \frac{\theta}{2} G_{x}+\sin ^{2} \frac{\theta}{2} G_{z}-\sin \theta G_{c}^{-} \\
G_{2} & =\sin ^{2} \frac{\theta}{2} G_{x}+\cos ^{2} \frac{\theta}{2} G_{z}+\sin \theta G_{c}^{-}
\end{aligned}
$$

for the difference resonance. We can easily see that the local invariant sum rules (3.105) are satisfied. Note that in addition to mixing the uncoupled invariants $G_{x}$ and $G_{z}$, an additional term, $G_{c}^{ \pm}$is picked up. This middle term can be related to the additional invariants that exist exactly on resonance (see [33]), which are mixed together by the phase $\phi$. These terms are given by

$$
\begin{aligned}
G_{c}^{+} & =-\frac{1}{2} J\left(e^{i \phi} v_{x}^{*} v_{-z}^{T}+e^{-i \phi} v_{x} v_{-z}^{\dagger}+e^{-i \phi} v_{-z}^{*} v_{x}^{T}+e^{i \phi} v_{-z} v_{x}^{\dagger}\right) J, \\
G_{c}^{-} & =-\frac{1}{2} J\left(e^{i \phi} v_{x}^{*} v_{z}^{T}+e^{-i \phi} v_{x} v_{z}^{\dagger}+e^{-i \phi} v_{z}^{*} v_{x}^{T}+e^{i \phi} v_{z} v_{x}^{\dagger}\right) J .
\end{aligned}
$$

Writing them out explicitly, we find

$$
G_{c}^{ \pm}=\left(\begin{array}{cc}
0_{2 \times 2} & \bar{G}_{c}^{ \pm} \\
\bar{G}_{c}^{ \pm T} & 0_{2 \times 2}
\end{array}\right)
$$

with their submatrices given by

$$
\bar{G}_{c}^{+}=\frac{1}{2}\left(\begin{array}{cc}
\frac{\left(-1+\alpha_{x} \alpha_{z}\right) \cos \phi+\left(\alpha_{x}+\alpha_{z}\right) \sin \phi}{\sqrt{\beta_{x} \beta_{z}}} & \sqrt{\frac{\beta_{z}}{\beta_{x}}}\left(\alpha_{x} \cos \phi+\sin \phi\right) \\
\sqrt{\frac{\beta_{x}}{\beta_{z}}}\left(\alpha_{z} \cos \phi+\sin \phi\right) & \sqrt{\beta_{x} \beta_{z}} \cos \phi
\end{array}\right),
$$


and

$$
\bar{G}_{c}^{-}=\frac{1}{2}\left(\begin{array}{cc}
-\frac{\left(\alpha_{x}-\alpha_{z}\right) \cos \phi+\left(1+\alpha_{x} \alpha_{z}\right) \sin \phi}{\sqrt{\beta_{x} \beta_{z}}} & \sqrt{\frac{\beta_{z}}{\beta_{x}}}\left(\cos \phi-\alpha_{x} \sin \phi\right) \\
-\sqrt{\frac{\beta_{x}}{\beta_{z}}}\left(\cos \phi+\alpha_{z} \sin \phi\right) & -\sqrt{\beta_{x} \beta_{z}} \sin \phi
\end{array}\right)
$$

for the sum and difference resonances respectively. Note that these additional coupling terms all contain a factor of $\cos \phi$ or $\sin \phi$. In the typical case, this term will oscillate many times around the ring. This gives the local oscillation in the beam matrix due to the coupling and is to be expected. When used to construct the global diffusion coefficient, however, this term will not contribute substantially. An exception to this is for the case where the integer parts of the degenerate tunes are equal which we discuss further later.

As a final remark, we point out that if the coupling angle $\theta$ is non-zero, then there is coupling throughout the entire ring. One may wonder ${ }^{5}$ how this is consistent with local coupling in which a certain region of the ring is coupled, but the ring is uncoupled everywhere outside that region. We see here that that situation requires "strong coupling", or more precisely, that it is not a resonant effect: to lowest order in our degenerate perturbation theory, there is global coupling, or no coupling at all.

\subsubsection{Integer/Half-integer Resonance}

Now consider the integer and half-integer resonances. The eigenvector is of the form (3.40). Consider the $x$ integer/half integer resonances. Then $v_{j 0}=v_{x}$ and $v_{k 0}=v_{-x}$. Constructing the invariant out of the eigenvector gives

$$
G_{1}=\cosh \theta G_{x}+\sinh \theta G_{c}
$$

where

$$
G_{c}=\left(\begin{array}{cccc}
\frac{\left(-1+\alpha_{x}^{2}\right) \cos \phi+2 \alpha_{x} \sin \phi}{\beta_{x}} & \alpha_{x} \cos \phi+\sin \phi & 0 & 0 \\
\alpha_{x} \cos \phi+\sin \phi & \beta_{x} \cos \phi & 0 & 0 \\
0 & 0 & 0 & 0 \\
0 & 0 & 0 & 0
\end{array}\right)
$$

\footnotetext{
${ }^{5}$ Thanks to Cristoph Steier for raising this point.
} 
In these expressions, the coupling angle $\theta$ and phase $\phi$ are the appropriate expression for the integer or half integer resonance as the case may be. Note that we could also obtain the additional coupling term $G_{c}$ by setting $\beta_{z}=\beta_{x}$ and $\alpha_{z}=\alpha_{x}$ in Eq.(3.97). Note that we only have two terms in these expressions for the invariant, versus three for the sum and difference resonances. This is because the invariants corresponding to $v_{x}$ and $v_{-x}$ are identical. For $z$ we just replace all the $x$ 's with $z$ 's.

Note that for an integer or half-integer resonance, the invariants remain block diagonal to the order we compute here. One could thus interpret the new invariant as defining new $\beta, \alpha$, and $\gamma$ rather than as a perturbed invariant. This allows us to compare our results to Courant and Snyder [22]. If we do this, we compare Eqs.(3.99) and (3.100) to Eq. (2.41) to derive a perturbed beta function

$$
\beta_{1}=\beta_{x}(\cosh \theta+\cos \phi \sinh \theta)
$$

which reduces to $\beta_{x}$ when the coupling is turned off. The value of $\phi$ varies around the ring via Eq. (3.86) from its initial value as given in Table 3.1. This is a well known effect referred to as a beta-beat, the periodic oscillation of the beta function resulting from a perturbation. Note that the initial value will be different for the integer and half-integer resonances. Also note that because $\cosh \theta>|\sinh \theta|, \beta_{1}$ will always be positive, although the range of the oscillations becomes large as the instability $(\theta \rightarrow \infty)$ is approached.

\subsection{Sum Rules}

Under some specific conditions, one can obtain a sum rule for the invariants. In particular, consider a 2-D coupled storage ring. Eq.(2.24) says that

$$
\mu_{1} G_{1}+\mu_{2} G_{2}=S
$$

In later sections, we will formulate a perturbation theory in which we start with an uncoupled, degenerate map and consider the effects of small coupling and small deviation from degeneracy. Let us write $\mu_{x 0}$ and $\mu_{z 0}$ for the phase advances at degeneracy 
(e.g. $\mu_{x 0}=-\mu_{z 0}$ for sum resonances, and $\mu_{x 0}=\mu_{z 0}$ for difference resonances) and $S_{0}$ for the effective Hamiltonian matrix at the degeneracy. At the degeneracy, then, we have

$$
\mu_{x 0} G_{x}+\mu_{z 0} G_{z}=S_{0}
$$

Breaking the degeneracy and adding a small coupling amount to adding small perturbations to the one-turn map. This will add small corrections to $S_{0}$, and $\mu_{x 0, z 0}$ to first order in the perturbations. The invariants $G_{1,2}$, however can have large changes depending on the perturbation, as will be clear in later sections. Then, taking (3.102) to lowest order and setting it equal to (3.103), we get

$$
\mu_{x 0} G_{1}+\mu_{z 0} G_{2}=\mu_{x 0} G_{x}+\mu_{z 0} G_{z}
$$

This is a sum rule for the invariants. For the case of the sum/difference resonance it says

$$
\begin{array}{ll}
G_{1}-G_{2}=G_{x}-G_{z} & \text { sum res. } \\
G_{1}+G_{2}=G_{x}+G_{z} & \text { dif. res. }
\end{array}
$$

For the integer resonance in the longitudinal motion, we have $\mu_{z 0}=0$, in which case this sum rule tells us that to lowest order, the invariant in the $x$-dimention is unchanged ${ }^{6}$,

$$
G_{1}=G_{x} \quad \text { int. } z \text { res. }
$$

Similarly,

$$
G_{2}=G_{z} . \quad \text { int. } x \text { res. }
$$

\footnotetext{
${ }^{6}$ One might be tempted to write the sum rule for the invariants as $\mu_{1} G_{1}+\mu_{2} G_{2}=\mu_{x} G_{x}+\mu_{z} G_{z}$ and note that near an integer $(x)$ resonance $\mu_{2} \approx \mu_{z}$ and $G_{2} \approx G_{z}$ and hence conclude that $G_{1}=\left(\mu_{x} / \mu_{1}\right) G_{x}$. This reasoning is incorrect since the sum rule is only valid to lowest order, and to lowest order $\mu_{1}=\mu_{x 0}=0$. Eq. (3.99) later shows that this flawed reasoning misses a term.
} 


\section{6 x-y coupling: skew quadrupole}

As our first example of an application of the formalism, we consider a skew quadrupole ${ }^{7}$. We focus on 4 -D $x_{\beta}-y_{\beta}$ phase space. The map is given by

$$
T_{\mathrm{sk}}=\left(\begin{array}{cccc}
1 & 0 & 0 & 0 \\
0 & 1 & \xi_{c} & 0 \\
0 & 0 & 1 & 0 \\
\xi_{c} & 0 & 0 & 1
\end{array}\right)
$$

so that the perturbation is given by

$$
P_{\mathrm{sk}}=\left(\begin{array}{cccc}
0 & 0 & 0 & 0 \\
0 & 0 & \xi_{c} & 0 \\
0 & 0 & 0 & 0 \\
\xi_{c} & 0 & 0 & 0
\end{array}\right)
$$

Computing the matrix elements, we find

$$
\begin{aligned}
r_{12 \xi} & =-\frac{i \xi_{c}}{2} \sqrt{\beta_{x} \beta_{y}} \\
r_{1-2 \xi} & =\frac{\xi_{c}}{2} \sqrt{\beta_{x} \beta_{y}}
\end{aligned}
$$

We now compute the coupling angle and phase for each of the resonances. Let us consider the difference resonance and suppose we have many skew coupling errors, with strength $\xi_{c}\left(s_{j}\right)$. Instead of computing the perturbation matrix, we will compute the matrix element directly, adding up the matrix elements from each perturbation. The resulting $r_{1-2}$ at position $s$ will be

$$
r_{1-2}=\sum_{j} \frac{\xi_{c}\left(s_{j}\right)}{2} \sqrt{\beta_{x}\left(s_{j}\right) \beta_{y}\left(s_{j}\right)} e^{i \Delta \psi\left(s_{j}\right)}
$$

\footnotetext{
${ }^{7}$ For more on the betatron coupled case, see [64]
} 
where $\Delta \psi\left(s_{j}\right)=\psi_{x s \rightarrow s_{j}}-\psi_{y s \rightarrow s_{j}}$ Taking a continuous limit and replacing $\xi_{c}\left(s_{i}\right)$ with a continuous $k\left(s^{\prime}\right)$ so that $\int_{s_{i}-\delta s}^{s_{i}+\delta s} d s^{\prime} k\left(s^{\prime}\right)=\xi_{c}\left(s_{i}\right)$ we get

$$
\xi=\left|\int_{s}^{s+C} d s^{\prime} \frac{k\left(s^{\prime}\right)}{2} \sqrt{\beta_{x}\left(s^{\prime}\right) \beta_{y}\left(s^{\prime}\right)} e^{i \Delta \psi\left(s^{\prime}\right)}\right|
$$

$\frac{\xi}{2 \pi}$ is the absolute value of the "coupling coefficient" derived by Guignard[31]. The coupling angle is

$$
\tan \theta=\frac{\xi_{-}}{2 \pi\left(\nu_{x}-\nu_{y}\right)}
$$

\subsubsection{Numerical Instability Plot}

In Figure 6.2, we give a numerical instability plot for a ring with an added skew quadrupole perturbation. For simplicity, we have set $\beta_{x}=\beta_{y}=1$ and $\alpha_{x}=\alpha_{y}=0$, and have set the value of $\xi_{c}$ in 3.109 to be 0.25 . The black regions represent values of the tunes $\nu_{x}$ and $\nu_{y}$ where the absolute values of an eigenvalue pair is not equal to 1. The large value for $\xi_{c}$ was chosen so that all the instabilities could be seen on the same plot. In particular, the integer and half integer stop-bands are narrower than that of the sum resonance, being second order in $\xi_{c}$. The structure given here should be thought of as the generic structure of instabilities. Later, when we consider cases of synchrobetatron coupling, we will see similar structures. However, in that case because the longitudinal beta function depends on $\nu_{z}$, so will the coupling parameters and hence the stop-band widths. This will perturb the shape of these instability plots, but the basic features are similar. When the perturbation has components in the on diagonal blocks, this will also slightly perturb the picture, as we see later in the case of dispersion at a crab cavity.

\section{7 x-z Coupling: Dispersion at an RF Cavity}

As our first example, we consider coupling due to dispersion at the RF cavity. The uncoupled longitudinal one turn map $M_{z}$ was given by (2.36). This was derived in physical coordinates. If there is dispersion at the cavity, we must transform the map 


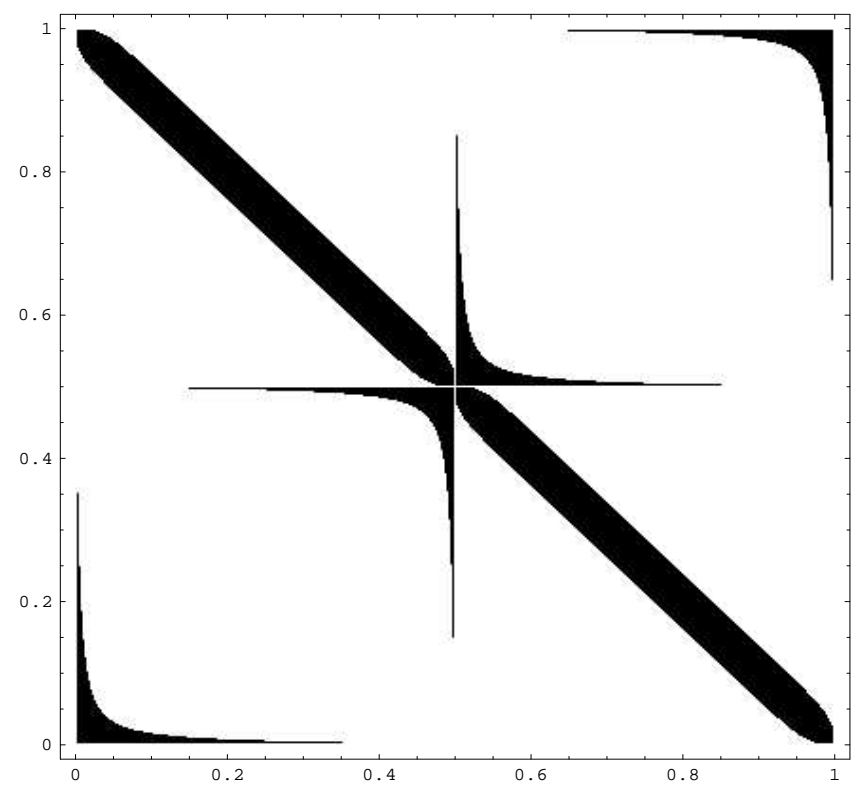

Figure 3.1: Instability plot for coupling due to skew quad with $\xi_{c}=0.25$. The horizontal axis is $\nu_{x}$ and the vertical is $\nu_{y}$.

into betatron coordinates and (2.29) is no longer block diagonal. The cavity map is transformed with $\mathcal{B} T_{\text {cav }} \mathcal{B}^{-1}$. We would like to pull out the part that causes the synchrotron oscillations and consider the extra part as the perturbation. To do this, we write

$$
\mathcal{B} T_{\mathrm{cav}} \mathcal{B}^{-1}=\left(1+P_{\mathrm{RF}}\right) T_{\mathrm{cav}}
$$

where $T_{\text {cav }}$ was given in (2.32), except here we work in the 4 -D space, so include a $2 \times 2$ identity matrix in the upper left block and 0's in the off-diagonal blocks. Using this prescription, we derive

$$
P_{\mathrm{RF}}=\frac{r}{a}\left(\begin{array}{cccc}
\eta \eta^{\prime} & -\eta^{2} & -\eta & 0 \\
\eta^{\prime 2} & -\eta \eta^{\prime} & -\eta^{\prime} & 0 \\
0 & 0 & 0 & 0 \\
-\eta^{\prime} & \eta & 0 & 0
\end{array}\right)
$$

From this matrix we construct the $r_{j k \xi}$ :

$$
r_{11}=-i \frac{r}{2 a} \mathcal{H}_{x}
$$




$$
\begin{aligned}
r_{1-1} & =\frac{r}{2 a \beta_{x}}\left[\left(i+\alpha_{x}\right) \eta+\beta_{x} \eta^{\prime}\right]^{2} \\
r_{12} & =i \frac{r}{2 a} \sqrt{\frac{\beta_{z}}{\beta_{x}}}\left[\left(i+\alpha_{x}\right) \eta+\beta_{x} \eta^{\prime}\right] \\
r_{1-2} & =-\frac{r}{2 a} \sqrt{\frac{\beta_{z}}{\beta_{x}}}\left[\left(i+\alpha_{x}\right) \eta+\beta_{x} \eta^{\prime}\right] \\
r_{22} & =r_{2-2}=0
\end{aligned}
$$

where $\mathcal{H}_{x}$ is defined in Eq. (4.36). From these we find $\xi, \Delta \mu, \phi$, and $\bar{\mu}$ for each resonance out of which we can construct the perturbed eigenvalues and eigenvectors.

\subsubsection{Sum/Difference Resonance}

Examining the perturbation (3.116) we see that the determinant of the off diagonal blocks is zero. From Appendix C, this tells us that the coupling parameters for the sum and difference resonances will be equal. Computing them, we find

$$
\xi_{ \pm}=\frac{r}{a} \sqrt{\mathcal{H}_{x} \beta_{z}}
$$

where the \pm indicates both the sum and difference resonance. If we expand this to lowest order in $\mu_{z}$, we find

$$
\xi_{ \pm}=\sqrt{\frac{\mathcal{H}_{x}}{a}}\left|\mu_{z}\right|^{3 / 2}
$$

Note that this goes to 0 at as $\mu_{z}$ goes to zero. This means that we expect the sum and difference resonances to become weaker for smaller synchrotron tune. For the splitting parameter we find

$$
\Delta \mu=\mu_{x}+\mu_{z}+\frac{r}{a} \mathcal{H}_{x}
$$

for the sum resonance and

$$
\Delta \mu=\mu_{x}-\mu_{z}+\frac{r}{a} \mathcal{H}_{x}
$$

for the difference resonance. The quantitiy $r$ is approximately $\mu_{z}^{2}$, so the third terms in these expressions can typically be ignored. For both sum and difference resonance, 
the average shift of the eigen-phase advance is

$$
\bar{\mu}=-\mu_{z}^{2} \frac{\mathcal{H}_{x}}{2 a}
$$

The phase for the sum resonance is

$$
\phi=\arg \left[-\left(i+\alpha_{x}\right) \eta+\beta_{x} \eta^{\prime}\right]
$$

while for the difference resonance

$$
\phi=\arg \left[i\left(i+\alpha_{x}\right) \eta+\beta_{x} \eta^{\prime}\right]
$$

To lowest order in $\mu_{z}$, the instability condition for the sum resonance is

$$
\sqrt{\frac{\mathcal{H}_{x}}{a}}\left|\mu_{z}\right|^{\frac{3}{2}}>\left|\mu_{x}+\mu_{z}\right|
$$

\subsubsection{Integer/Half-integer Resonance}

We now consider the integer and half integer resonances. Near the integer $x$ resonance, we find that the coupling parameter is proportional to $\mu_{z}^{4}$. There is still in fact an instability for $\nu_{x}$ near an integer, but it is extremely weak, with a width of order $\frac{\mathcal{H}_{x}}{a} \mu_{z}^{4}$. We do not consider this resonance further.

For the half integer resonance in $\mu_{x}$, we find

$$
\begin{aligned}
\xi & =\mu_{z}^{2} \frac{\mathcal{H}_{x}}{a} \\
\Delta \mu & =2\left(\mu_{x}-\pi\right)-\frac{\mathcal{H}_{x}}{a} \mu_{z}^{2} \\
\phi & =2 \arg \left(\mathcal{G}_{x}+i \eta\right)
\end{aligned}
$$

where we define

$$
\mathcal{G}_{x}=\eta^{\prime} \beta_{x}+\eta \alpha_{x}
$$


We find that there is an instability when

$$
\frac{\mathcal{H}_{x}}{a} \mu_{z}^{2}>\mu_{x}-\pi
$$

This says that there is an instability for $\mu_{x}>\pi$ with a width of $\frac{\mathcal{H}_{x}}{a} \mu_{z}^{2}$. Note that these results are identical to those we would obtain by using the 5th row in Table 3.1 (first order calculation). The other terms are higher order in $\mu_{z}$ and have been ignored.

For the integer resonance in $\mu_{z}$ ( $\mu_{z}$ near 0 , as is typically the case), we get

$$
\begin{aligned}
\xi & =\left|\frac{\mu_{z}^{3} \mathcal{H}_{x}}{2 a} \cot \frac{\mu_{x}}{2}\right| \\
\Delta \mu & =2 \mu_{z}-\frac{\left|\mu_{z}\right|^{3} \mathcal{H}_{x}}{2 a} \cot \left(\frac{\mu_{x}}{2}\right) \\
\phi & =0 .
\end{aligned}
$$

Here we find that $\mu_{2}$ is given by

$$
\mu_{2}=\mu_{z} \sqrt{1+\mu_{z}^{2} \frac{\mathcal{H}_{x}}{2 a} \cot \left(\frac{\mu_{x}}{2}\right)}
$$

From this expression, we can see that there is no instability for the $\mu_{z}$ integer resonance. The quantity inside the square root could become negative only in two cases. One case is where the perturbation $P_{\mathrm{RF}}$ is not small and hence perturbation theory no longer applies. The other case is if $\mu_{x}$ is near 0 or $2 \pi$ in which case we are in the region of overlap between two different resonances in which we may need to consider the other resonances. Numerical calculation confirms that there is in fact no instability for small synchrotron tune, regardless of the values of the other parameters. 


\begin{tabular}{|lccccc|}
\hline reso. & condition & $\Delta \mu(\bmod 2 \pi)$ & $\xi$ & $\phi$ & $\bar{\mu}$ \\
\hline \hline $\operatorname{sum}$ & $\mu_{x}+\mu_{z}=2 \pi n$ & $\mu_{x}+\mu_{z}+\frac{r}{a} \mathcal{H}_{x}$ & $\sqrt{\frac{\mathcal{H}_{x}}{a}}\left|\mu_{z}\right|^{\frac{3}{2}}$ & $\arg \left(-\left(i+\alpha_{x}\right) \eta+\beta_{x} \eta^{\prime}\right)$ & $-\mu_{z}^{2} \frac{\mathcal{H}_{x}}{2 a}$ \\
\hline dif. & $\mu_{x}-\mu_{z}=2 \pi n$ & $\mu_{x}-\mu_{z}+\frac{r}{a} \mathcal{H}_{x}$ & $\sqrt{\frac{\mathcal{H}_{x}}{a}}\left|\mu_{z}\right|^{\frac{3}{2}}$ & $\arg \left(i\left(i+\alpha_{x}\right) \eta+\beta_{x} \eta^{\prime}\right)$ & $-\mu_{z}^{2} \frac{\mathcal{H}_{x}}{2 a}$ \\
\hline $\operatorname{int}(x)$ & $\mu_{x}=2 \pi n$ & $2 \mu_{x}+\mathcal{O}\left(\frac{\mathcal{H}_{x}}{a} \mu_{z}^{4}\right)$ & $\mathcal{O}\left(\frac{\mathcal{H}_{x}}{a} \mu_{z}^{4}\right)$ & $2 \arg \left(\mathcal{G}_{x}+i \eta\right)$ & 0 \\
\hline $\operatorname{int}(z)$ & $\mu_{z}=2 \pi n$ & $2 \mu_{z}-\frac{\mu_{z} \mathcal{H}_{x}}{2 a} \cot \left(\frac{\mu_{x}}{2}\right)$ & $\frac{\mu_{s} \mathcal{H}_{x}}{2 a}\left|\cot \left(\frac{\mu_{x}}{2}\right)\right|$ & 0 & 0 \\
\hline$\frac{1}{2}-\operatorname{int}(x)$ & $\mu_{x}=\pi(2 n+1)$ & $2\left(\mu_{x}-\pi\right)-\frac{\mathcal{H}_{x}}{a} \mu_{z}^{2}$ & $\mu_{z}^{2} \frac{\mathcal{H}_{x}}{a}$ & $2 \arg \left(\mathcal{G}_{x}+i \eta\right)$ & 0 \\
\hline
\end{tabular}

Table 3.2: Resonances for dispersive RF cavity

\subsection{Crab Cavity}

As our third example, we consider a single crab cavity[68]. The map for the crab cavity is given by

$$
T_{\text {crab }}=\left(\begin{array}{cccc}
1 & 0 & 0 & 0 \\
0 & 1 & \xi_{c} & 0 \\
0 & 0 & 1 & 0 \\
\xi_{c} & 0 & 0 & 1
\end{array}\right),
$$

where $\xi_{c}$ gives the strength of the cavity. In the case where the crab cavity is used to correct for a half crossing angle $\Phi$ at the interaction point of a collider [60], assuming no resonance, $\xi_{c}$ is related to that crossing angle by

$$
\xi_{c}= \begin{cases}\frac{2 \Phi \sin \left(\pi \nu_{x}\right)}{\sqrt{\beta_{k} \beta_{x}^{*}}} & \text { single crab cavity } \\ \frac{\Phi}{\sqrt{\beta_{k} \beta_{x}^{*}}} & \text { crab cavity pair }\end{cases}
$$

where $\beta_{k}$ is the beta function at the crab cavity and $\beta_{x}^{*}$ is the beta function at the interaction point. For a pair of crab cavities, one positions them symmetrically about the interaction point such that there is a total of a $\pi$ phase shift between them and the second cancels out the effect of the first in the rest of the ring. The expression given here for a single crab cavity comes from computing the angle at the IP due to a change in closed orbit, assuming no resonance. Near a resonance, this formula needs to be reconsidered. Indeed, it is the purpose of this paper to determine the effect on beam dynamics due to coupling near a resonance. We include this formula because in practice, the value of $\xi_{c}$ may be chosen assuming the machine is away from all resonances. 
We here consider the case of a single crab cavity. Suppose we insert a crab cavity in the ring at position $s_{2}$. The RF cavity is assumed to be located at $s_{c}$ so that the partial momentum compaction factor as given in (2.35) involves integration from $s_{c}$ to $s_{2}$.

Transforming (3.135) into betatron coordinates, we get for the perturbation

$$
P_{\text {crab }}=\xi_{c}\left(\begin{array}{cccc}
-\eta & 0 & 0 & -\eta^{2} \\
-2 \eta^{\prime} & \eta & 1 & -\eta \eta^{\prime} \\
\eta \eta^{\prime} & -\eta^{2} & -\eta & 0 \\
1 & 0 & 0 & \eta
\end{array}\right)
$$

Note that the perturbation is proportional to the crab cavity strength $\xi_{c}$. Note also that $\eta$ here now refers to the dispersion at the crab cavity while we assume the dispersion at the RF acceleration cavity to vanish.

First we compute the $r_{j k \xi}$ :

$$
\begin{aligned}
r_{11 \xi} & =i \xi_{c} \mathcal{G}_{x} \\
r_{1-1 \xi} & =-\xi_{c}\left(\left(i+\alpha_{x}\right) \eta+\beta_{x} \eta^{\prime}\right) \\
r_{12 \xi} & =-i \xi_{c} \frac{\left(i+\alpha_{x}\right)\left(-i+\alpha_{z}\right) \eta^{2}+\beta_{x}\left(\beta_{z}+\left(-i+\alpha_{z}\right) \eta \eta^{\prime}\right)}{2 \sqrt{\beta_{x} \beta_{z}}} \\
r_{1-2 \xi} & =\xi_{c} \frac{\left(i+\alpha_{x}\right)\left(i+\alpha_{z}\right) \eta^{2}+\beta_{x}\left(\beta_{z}+\left(i+\alpha_{z}\right) \eta \eta^{\prime}\right)}{2 \sqrt{\beta_{x} \beta_{z}}} \\
r_{22 \xi} & =i \xi_{c} \eta \alpha_{z} \\
r_{2-2 \xi} & =\xi_{c}\left(-i+\alpha_{z}\right) \eta
\end{aligned}
$$

The rest of the $r_{j k}$ can be gotten using (3.19) and (3.20).

Next, we construct $\xi, \Delta \mu, \phi$ and $\bar{\mu}$ for each of the resonances and from these compute $\theta$ and $\mu_{1}$ and $\mu_{2}$.

\subsubsection{Sum/Difference Resonance}

In this case, examining the perturbation matrix (3.137) we find that the determinant of the off-diagonal submatrices are not zero. We thus expect a difference between the 
coupling parameters for the sum and difference resonances. Indeed, we find

$$
\xi_{ \pm}=\xi_{c} \sqrt{\frac{a \beta_{x}}{\mu_{s}} \mp 2 \eta^{2}}
$$

Note that $\xi_{-}^{2}-\xi_{+}^{2}=4 \xi_{c}^{2} \eta^{2}$ which is consistent with Eq. (C.5) from Appendix C. We see that in the case that the dispersion at the crab cavity is zero, the coupling parameter is inversely proportional to the square root of the synchrotron tune. Thus, we expect the sum and difference resonances to get stronger for small synchrotron tune. The splitting parameter is given by

$$
\Delta \mu=\mu_{x}+\mu_{z}+\xi_{c}\left(\mathcal{G}_{x}+\eta \alpha_{z}\right)
$$

for the sum resonance, and

$$
\Delta \mu=\mu_{x}-\mu_{z}+\xi_{c}\left(\mathcal{G}_{x}-\eta \alpha_{z}\right)
$$

for the difference resonance. $\mathcal{G}_{x}$ was defined in Eq. (3.129). The phase $\phi$ is given by

$$
\phi=\arg \left[\frac{a \beta_{x}}{\mu_{s}}-\eta^{2}+i \eta \mathcal{G}_{x}\right]
$$

for the sum resonance and

$$
\phi=\arg \left[-\eta \mathcal{G}_{x}-i\left(\frac{a \beta}{\mu_{s}}-\eta^{2}\right)\right]
$$

for the difference resonance. For the case of no dispersion at the crab cavity, the difference resonance has $\phi=-\frac{\pi}{2}$ and the sum resonance has $\phi=0$. The average shift in the phase advance, $\bar{\mu}$ is given by

$$
\bar{\mu}=\xi_{c}\left(\mathcal{G}_{x} \pm \eta \alpha_{z}\right)
$$

with the plus sign for the difference resonance and the minus sign for the sum resonance. 
For the sum resonance, we find an instability for

$$
\xi_{c} \sqrt{\frac{a \beta_{x}}{\mu_{s}}-2 \eta^{2}}>\mu_{x}+\mu_{z}+\xi_{c}\left(\mathcal{G}_{x}+\eta \alpha_{z}\right)
$$

\subsubsection{Integer/Half-integer Resonance}

For the $x$ integer resonance the coupling parameter is

$$
\xi^{2}=4 \beta_{x} \mathcal{H}_{x} \xi_{c}^{2}+\frac{4 a \beta_{x} \mathcal{G}_{x}}{\mu_{s}^{2}} \xi_{c}^{3}+\frac{a^{2} \beta_{x}^{2}}{\mu_{s}^{4}} \xi_{c}^{4}
$$

The splitting parameter is

$$
\Delta \mu=2 \mu_{x}+2 \xi_{c} \mathcal{G}+\xi_{c}^{2} \frac{a \beta_{x}}{\mu_{s}^{2}}
$$

and the phase, to lowest order in $\mu_{z}$ and $\xi_{c}$ is

$$
\phi=\pi
$$

Out of $\xi$ and $\Delta \mu$, we construct

$$
\mu_{1}^{2}=\left(\mu_{x}+\xi_{c} \mathcal{G}_{x}\right)^{2}+\left(\mu_{x}+\xi_{c} \mathcal{G}_{x}\right) \frac{a \beta_{x}}{\mu_{s}^{2}} \xi_{c}^{2}-\frac{a \beta_{x} \mathcal{G}_{x}}{\mu_{s}^{2}} \xi_{c}^{3}-\beta_{x} \mathcal{H}_{x} \xi_{c}^{2}
$$

From this, we can see that for $\mu_{x}<0$ (or close to $2 \pi$ ) and for the case of no dispersion, we can have an instability if

$$
\frac{a \beta_{x}}{\mu_{s}^{2}} \xi_{c}^{2}>\left|\mu_{x}\right| \quad\left(\mu_{x}<0\right)
$$

At larger $\mu_{z}$ and with non-negligable dispersion at the crab cavity and for the case $\mathcal{G}_{x}=0$, we find an instability for

$$
\eta \xi_{c}>\left|\mu_{x}\right|
$$

for both positive and negative $\mu_{x}$. 
For the half integer resonance in $x$, we find a coupling parameter

$$
\xi^{2}=4 \beta_{x} \mathcal{H}_{x} \xi_{c}^{2}-a \beta_{x} \mathcal{G}_{x} \xi_{c}^{3}+\frac{1}{16} a^{2} \beta_{x}^{2} \xi_{c}^{4}
$$

The splitting parameter is

$$
\Delta \mu=2\left(\mu_{x}-\pi\right)+2 \xi_{c} \mathcal{G}_{x}-\frac{1}{4} a \beta_{x} \xi_{c}^{2}
$$

and the phase is

$$
\phi=\arg \left(\frac{1}{8} a \beta_{x} \xi_{c}^{2}-\mathcal{G}_{x} \xi_{c}-i \eta\right)
$$

which vanishes when there is no dispersion at the crab cavity. Computing $\Delta \mu^{2}-\xi^{2}$ and noting the cancellation of terms, we find

$$
\begin{aligned}
\left(\mu_{1}-\pi\right)^{2}= & {\left[\left(\mu_{x}-\pi\right)+\xi_{c} \mathcal{G}_{x}\right]^{2}-\left[\left(\mu_{x}-\pi\right)+2 \xi_{c} \mathcal{G}_{x}\right] \frac{a \beta_{x}}{4} \xi_{c}^{2} } \\
& -\beta_{x} \mathcal{H}_{x} \xi_{c}^{2}+\frac{1}{4} a \beta_{x} \mathcal{G}_{x} \xi_{c}^{3}
\end{aligned}
$$

For the case of $\mathcal{G}_{x}=0$, we can have an instability for

$$
\left(\mu_{x}-\pi\right) \frac{a \beta_{x}}{4} \xi_{c}^{2}+\eta^{2} \xi_{c}^{2}>\left(\mu_{x}-\pi\right)^{2}
$$

Next for the integer resonance in $z$, we find

$$
\begin{gathered}
\xi^{2}=4 \eta^{2} \xi_{c}^{2}+\frac{a^{2} \beta_{x}^{2} \cot ^{2}\left(\frac{\mu_{x}}{2}\right)}{4 \mu_{s}^{2}} \xi_{c}^{4} \\
\Delta \mu=2 \mu_{z}-\frac{1}{2} \frac{a \beta_{x}}{\mu_{s}} \cot \left(\frac{\mu_{x}}{2}\right) \xi_{c}^{2}
\end{gathered}
$$

and

$$
\phi=\arg \left[\frac{a \beta_{x} \cot \left(\frac{\mu_{x}}{2}\right)}{4 \mu_{s}} \xi_{c}^{2}-i \eta \xi_{c}\right]
$$

For the case where $\eta=0$, the phase $\phi$ is 0 for $\mu_{x}>\pi$ and $\pi$ for $\mu_{x}<\pi$. Computing $\Delta \mu^{2}-\xi^{2}$, we find

$$
\mu_{2}^{2}=\mu_{s}^{2}+\frac{a \beta_{x}}{4} \cot \left(\frac{\mu_{x}}{2}\right) \xi_{c}^{2}-\eta^{2} \xi_{c}^{2}
$$


From this, we can see that there is an instability if

$$
\frac{a \beta_{x}}{4} \cot \left(\frac{\mu_{x}}{2}\right) \xi_{c}^{2}-\eta^{2} \xi_{c}^{2}<\mu_{s}^{2}
$$

When $\eta=0$, this requires $\mu_{x}>\pi$, i.e. the horizontal betatron tune is above a half-integer. Increasing $\eta$ moves this instability to values of $\mu_{x}$ less than $\pi$.

Because in practice the synchrotron phase will not be near $\pi$, we do not consider the $\nu_{z}$ half-integer resonance.

We summarize the results for $\xi, \Delta \mu$, and $\phi$ for each of the resonances in Table 3.3 .

Table 3.3: Resonances for Crab Cavity

\begin{tabular}{|lccccc}
\hline reso. & condition & $\Delta \mu(\bmod 2 \pi)$ & $\xi^{2}$ & $\phi$ & $\bar{\mu}$ \\
\hline \hline sum & $\mu_{x}+\mu_{z}=2 \pi n$ & $\mu_{x}+\mu_{z}+\xi_{c}\left(\mathcal{G}_{x}+\eta \alpha_{z}\right)$ & $\xi_{c}^{2}\left(\frac{a \beta_{x}}{\mu_{s}}-2 \eta^{2}\right)$ & $\arg \left(\frac{a \beta_{x}}{\mu_{s}}-\eta^{2}+i \eta \mathcal{G}_{x}\right)$ & $\xi_{c}\left(\mathcal{G}_{x}-\eta \alpha_{z}\right)$ \\
\hline $\operatorname{diff.}$ & $\mu_{x}-\mu_{z}=2 \pi n$ & $\mu_{x}-\mu_{z}+\xi_{c}\left(\mathcal{G}_{x}-\eta \alpha_{z}\right)$ & $\xi_{c}^{2}\left(\frac{a \beta_{x}}{\mu_{s}}+2 \eta^{2}\right)$ & $\arg \left(-\eta \mathcal{G}_{x}-i\left(\frac{a \beta}{\mu_{s}}-\eta^{2}\right)\right)$ & $\xi_{c}\left(\mathcal{G}_{x}+\eta \alpha_{z}\right)$ \\
\hline $\operatorname{int}(x)$ & $\mu_{x}=2 \pi n$ & $2 \mu_{x}+2 \xi_{c} \mathcal{G}_{x}+\xi_{c}^{2} \frac{a \beta_{x}}{\mu_{s}^{2}}$ & $4 \beta_{x} \mathcal{H}_{x} \xi_{c}^{2}+\frac{4 a \beta_{x} \mathcal{G}_{x}}{\mu_{s}^{2}} \xi_{c}^{3}+\frac{a^{2} \beta_{x}^{2}}{\mu_{s}^{4}} \xi_{c}^{4}$ & $\pi$ & 0 \\
\hline $\operatorname{int}(z)$ & $\mu_{z}=2 \pi n$ & $2 \mu_{z}-\frac{1}{2} \frac{a \beta_{x}}{\mu_{s}} \cot \left(\frac{\mu_{x}}{2}\right) \xi_{c}^{2}$ & $4 \eta^{2} \xi_{c}^{2}+\frac{a^{2} \beta_{x}^{2} \cot ^{2}\left(\frac{\mu_{x}}{2}\right)}{4 \mu_{s}^{2}} \xi_{c}^{4}$ & $\arg \left(\frac{a \beta_{x} \cot \left(\frac{\mu_{x}}{2}\right)}{4 \mu_{s}} \xi_{c}^{2}-i \eta \xi_{c}\right)$ & 0 \\
\hline$\frac{1}{2}-\operatorname{int}(x)$ & $\mu_{x}=\pi(2 n+1)$ & $2\left(\mu_{x}-\pi\right)+2 \xi_{c} \mathcal{G}_{x}-\frac{1}{4} a \beta_{x} \xi_{c}^{2}$ & $4 \beta_{x} \mathcal{H}_{x} \xi_{c}^{2}-a \beta_{x} \mathcal{G}_{x} \xi_{c}^{3}+\frac{1}{16} a^{2} \beta_{x}^{2} \xi_{c}^{4}$ & $\arg \left(\frac{1}{8} a \beta_{x} \xi_{c}^{2}-\mathcal{G}_{x} \xi_{c}-i \eta\right)$ & 0 \\
\hline
\end{tabular}




\section{Chapter 4}

\section{Constant Damping/Diffusion}

In this chapter, we consider the case where the damping and diffusion coefficients are constant. An important application of this case is the example of diffusion and damping due to synchrotron radiation. We apply the general theory to the uncoupled case, yielding the results of Sands for the case of synchrotron radiation damping/diffusion.

In this case, we have

$$
b_{i}=B_{i k} z_{k}
$$

and we take this damping matrix $B$ and the diffusion matrix $D$ to be independent of $\vec{z}$. The result for the moment equation is then

$$
\Sigma(s+C)=M \Sigma(s) M^{T}-M \bar{B} \Sigma(s) M^{T}-M \Sigma(s) \bar{B}^{T} M^{T}+\bar{D}(s)
$$

where $\bar{B}$ and $\bar{D}$ are global damping and diffusion matrices determined by adding up local damping and diffusion coefficients and transforming them appropriately to the observation position. We can express $\bar{D}$ in terms of the integral

$$
\bar{D}(s)=\int_{s}^{s+C} d s^{\prime} T_{s^{\prime} \rightarrow s+C} D\left(s^{\prime}\right) T_{s^{\prime} \rightarrow s+C}^{T} .
$$

Physically, $\int D\left(s^{\prime}\right) d s^{\prime}$ over a storage ring element gives the contribution of diffusion to the $\Sigma$ second moments due to this element. This contribution is then transferred to position $s$ by the map $T_{s^{\prime} \rightarrow s+C}$ to give the integrated $\bar{D}$. 
Now we can determine the evolution equation for the invariants. We find that it will be convenient to define damping and diffusion coefficients for the eigen-invariants $b_{i}$ and $d_{i}$. The equilibrium emittances are then given as the ratio $d_{i} / 4 b_{i}$. To find the emittances, we need to find the invariants of the one-turn map, out of which we build the damping and diffusion coefficients.

To find the exact equilibrium distribution, we stipulate in Eq.(4.2) that $\Sigma(s+C)=$ $\Sigma(s)=\Sigma_{\text {eq }}(s)$. This equation can be solved for $\Sigma_{\text {eq }}$ directly using eigenanalysis $[37,32]$. For our purposes here, however, we assume that the distribution stays a function of the invariants. The average value of the invariant is given by

$$
\left\langle g_{a}\right\rangle=\operatorname{Tr}\left(G_{a} \Sigma\right), \quad a=1,2,3
$$

Using (4.4), (2.18), and (4.2), along with the cyclic property of the trace, we obtain the evolution equation for $\left\langle g_{a}\right\rangle$,

$$
\left\langle g_{a}\right\rangle(s+C)-\left\langle g_{a}\right\rangle(s)=-2 \operatorname{Tr}\left(\bar{B} \Sigma G_{a}\right)+\operatorname{Tr}\left(G_{a} \bar{D}\right) .
$$

We then use (2.27), (2.19), and $U^{T} G_{a} U=J H_{a} J$ (using the symplectic property of $U)$ to obtain, after some algebra,

$$
\left\langle g_{a}\right\rangle(s+C)-\left\langle g_{a}\right\rangle(s)=-2 \chi_{a}\left\langle g_{a}\right\rangle+\bar{d}_{a}
$$

where the global eigenmode damping decrements per turn $\chi_{a}$ are given by

$$
\chi_{a}=\bar{A}_{a a}+\bar{A}_{-a-a}, \quad a=1,2,3,
$$

where

$$
\bar{A}=U^{-1} \bar{B} U
$$

and the one-turn eigenmode diffusion coefficients $\bar{d}_{a}$ are

$$
\bar{d}_{a}=\operatorname{Tr}\left(G_{a} \bar{D}\right), \quad a=1,2,3 .
$$


From (4.6), we see that the equilibrium values of the invariants are given by

$$
\left\langle g_{a}\right\rangle_{\mathrm{eq}}=\frac{\bar{d}_{a}}{2 \chi_{a}}, \quad a=1,2,3 .
$$

It may be useful to express the global damping decrements $\chi_{a}$ and the global diffusion coefficients $\bar{d}_{a}$ in an integral form. Writing out (4.8) explicitly gives

$$
\begin{aligned}
\bar{A} & =U^{-1}(s) \bar{B}(s) U(s) \\
& =\int_{s}^{s+C} U^{-1}\left(s^{\prime}\right) B\left(s^{\prime}\right) U\left(s^{\prime}\right) d s^{\prime} \\
& \equiv \oint A\left(s^{\prime}\right) d s^{\prime}
\end{aligned}
$$

where we have used the fact that the $U$ matrix of eigenvectors transforms as $U(s)=$ $T_{s \rightarrow s^{\prime}} U\left(s^{\prime}\right)$, and defined the local $A$ matrix in terms of the local damping matrix as ${ }^{1}$

$$
A\left(s^{\prime}\right)=U^{-1}\left(s^{\prime}\right) B\left(s^{\prime}\right) U\left(s^{\prime}\right)
$$

The matrix $\bar{A}$ is independent of the observation position $s$, although $\bar{B}$ in general depends on $s$. We can thus write the global damping decrements as

$$
\chi_{a}=\oint\left[A_{a a}\left(s^{\prime}\right)+A_{-a-a}\left(s^{\prime}\right)\right] d s^{\prime} .
$$

We refer to the integrand as the local damping coefficient and write

$$
b_{a}=A_{a a}+A_{-a-a}
$$

so that

$$
\chi_{a}=\oint b_{a}\left(s^{\prime}\right) d s^{\prime}
$$

For the diffusion coefficients, we use (4.3), (4.9), and the transformation property

\footnotetext{
${ }^{1}$ The matrix $A$ depends on the overall phases of the eigenvectors $v_{k}$ in $U$. However, the diagonal elements are independent of these phases, and only the diagonal elements enter the evolution equations for the invariants.
} 
of the invariant $G_{a}\left(s^{\prime}\right)=T_{s^{\prime} \rightarrow s+C}^{T} G_{a}(s) T_{s^{\prime} \rightarrow s+C}$ to derive

$$
\bar{d}_{a}=\oint \operatorname{Tr}\left[G_{a}\left(s^{\prime}\right) D\left(s^{\prime}\right)\right] d s^{\prime} \equiv \oint d_{a}\left(s^{\prime}\right) d s^{\prime}
$$

where we have defined the local diffusion coefficient

$$
d_{a}=\operatorname{Tr}\left[G_{a} D\right]
$$

The global diffusion coefficients $\bar{d}_{a}$ are independent of $s$. It follows that the equilibrium emittances $\frac{1}{2}\left\langle g_{a}\right\rangle_{\text {eq }}$ are also global quantities, independent of $s$.

Thus, to find the equilibrium emittance, we must find the local invariants $G_{a}$ and the $U$ matrix constructed from the eigenvectors of $M$ using (2.16) and (2.19). Given these and local damping and diffusion matrices $B$ and $D$, we find the local damping and diffusion coefficients $b_{a}$ and $d_{a}$ from (4.12), (4.14), and (4.17). Finally, we integrate these around the ring to find the global damping decrements and global diffusion coefficients which give us the equilibrium emittances.

\subsection{Sum Rules}

The sum of the local damping coefficients gives the trace of $A(s)$. From this we derive

$$
\begin{aligned}
b_{1}+b_{2}+b_{3} & =\operatorname{Tr}\left(U^{-1} B U\right) \\
& =\operatorname{Tr}(B) \\
& =b_{x}+b_{y}+b_{z},
\end{aligned}
$$

where $b_{x}, b_{y}$, and $b_{z}$ are the damping coefficients in uncoupled coordinates. Thus, regardless of the details of the Hamiltonian dynamics (contained in $U(s)$ ), the sum of the $b_{a}$ is unchanged. This is a local sum rule for the damping coefficients. Integrating this equation around the ring, we get a global sum rule,

$$
\chi_{1}+\chi_{2}+\chi_{3}=\chi_{x}+\chi_{y}+\chi_{z}
$$


where $\chi_{x, y, z}$ are the global damping decrements for the uncoupled case. Equation (4.19) is a manifestation of the well-known Robinson theorem [65].

Let us recall that we also derived invariant sum rules in Chapter 3. The local diffusion coefficients $d_{a}$ are given by $d_{a}=\operatorname{Tr}\left(G_{a} D\right)$, where $D$ is the diffusion matrix. Multiplying the sum rules on the right by the matrix $D$ and then taking a trace gives sum rules for the local diffusion coefficients. The results for the sum/difference and integer resonances are

$$
\begin{aligned}
d_{1}-d_{2} & =d_{x}-d_{z}= & \text { invariant } & \text { sum res. } \\
d_{1}+d_{2} & =d_{x}+d_{z}= & \text { invariant } & \text { dif. res. } \\
d_{1} & =d_{x} & & \text { int } z \text { res. } \\
d_{2} & =d_{z} & & \text { int. } x \text { res. }
\end{aligned}
$$

We can also integrate this equation around the ring to get sum rules for the global diffusion coefficients,

$$
\begin{aligned}
\bar{d}_{1}-\bar{d}_{2} & =\bar{d}_{x}-\bar{d}_{z}= & \text { invariant } & \text { sum res. } \\
\bar{d}_{1}+\bar{d}_{2} & =\bar{d}_{x}+\bar{d}_{z}= & \text { invariant } & \text { dif. res. } \\
\bar{d}_{1} & =\bar{d}_{x} & \text { int } z \text { res. } & \\
\bar{d}_{2} & =\bar{d}_{z} & \text { int. } x \text { res. } &
\end{aligned}
$$

where the $\bar{d}_{a}$ are the integrated $d_{a}$ as given in (4.16).

From the sum rule (4.21) for sum and difference resonances (global version), and using (4.10), we have

$$
\begin{array}{ll}
\chi_{1}\left\langle g_{1}\right\rangle_{\mathrm{eq}}-\chi_{2}\left\langle g_{2}\right\rangle_{\mathrm{eq}}=\text { invariant } & \text { sum res. } \\
\chi_{1}\left\langle g_{1}\right\rangle_{\mathrm{eq}}+\chi_{2}\left\langle g_{2}\right\rangle_{\mathrm{eq}}=\text { invariant } & \text { dif. res. }
\end{array}
$$

Since $\chi_{1,2}$ must be positive for stable motion, it follows that this sum rule imposes a stability condition for particle motion. For example, in case of coupling between the two transverse betatron motions, Eq.(4.22) implies that the motion is stable near a difference resonance and possibly unstable near a sum resonance. This is 
a familiar result involving the sum rule of equilibrium beam emittances [22]. The present formalism therefore contains in one framework the Robinson sum rule and the emittance sum rule near linear resonances.

In the case where the coupling near a sum/difference resonance occurs in synchrobetatron space, and the operation is above transition, we find that $\mu_{z}=-\mu_{s}$ (see later) where $\mu_{s}$ is the usual (positive) synchrotron phase advance per turn. Thus, in terms of $\mu_{s}$, there is a sign reversal in the definition of degeneracy so that a sum resonance has $\mu_{x 0}=\mu_{s}$ and difference resonance has $\mu_{x 0}=-\mu_{s}$. In terms of $\mu_{s}$, then, stability applies near a sum resonance and instability occurs near a difference resonance. This is also a familiar result [12], associated with the longitudinal negative mass above transition. In the present paper, however, we make the choice to relate our definitions of resonance to $\nu_{z}$ so that as in the case of $x-y$ coupling, the difference resonance is stable and the sum resonance is unstable. This has the advantage of permitting a uniform treatment of synchrobetatron coupling and transverse betatron coupling. To reiterate, by "sum resonance", we mean $\nu_{x}+\nu_{z}$ is near an integer, and for a "difference resonance", $\nu_{x}-\nu_{z}$ is near an integer.

\subsection{Example: Synchrotron Radiation}

Our example of a process with constant damping and diffusion coefficients will be synchrotron radiation in an electron storage ring.

We find the damping and diffusion coefficients using Eqs. (2.67) and (2.68) where $g(\Phi)$ is the spectrum of emitted radiation with $\Phi=\omega$, the frequency of a given photon.

Ignoring the opening angle effect, the diffusion due to synchrotron radiation only happens in the coordinate $\delta$, so that

$$
\delta \vec{z}=(0,0,0,-\hbar \omega)
$$

The specific frequency of any given photon is random, but distributed according to the frequency spectrum of classical radiation. The frequency spectrum from a bending 
magnet is given by $S\left(\omega / \omega_{c}\right)$ with [36]

$$
S(\xi)=\frac{9 \sqrt{3}}{8 \pi} \xi \int_{\xi}^{\infty} K_{5 / 3} \bar{\xi} d \bar{\xi}
$$

where $K_{5 / 3}$ is the Bessel Function of order $5 / 3$ and

$$
\omega_{c}=\frac{3}{2} \frac{c \gamma^{3}}{\rho}
$$

Thus, we take $g(\Phi)$ to be $S(\omega)$. The other quantity we need is $\dot{n}$, the number of kicks per unit time which is just the number of photons emitted per unit time. This is given by

$$
\dot{n}=\frac{5 \alpha_{0} c \gamma}{2 \sqrt{3}}
$$

with $\alpha_{0}$ the fine structure constant.

For the diffusion and damping coefficients, we will need the quantities,

$$
\langle\omega\rangle=\frac{8}{15 \sqrt{3}} \omega_{c}
$$

and

$$
\left\langle\omega^{2}\right\rangle=\frac{11}{27} \omega_{c}^{2}
$$

Putting these together, we find

$$
D(s)=\left(\begin{array}{cccccc}
0 & 0 & 0 & 0 & 0 & 0 \\
0 & 0 & 0 & 0 & 0 & 0 \\
0 & 0 & 0 & 0 & 0 & 0 \\
0 & 0 & 0 & 0 & 0 & 0 \\
0 & 0 & 0 & 0 & 0 & 0 \\
0 & 0 & 0 & 0 & 0 & d(s)
\end{array}\right)
$$

where

$$
d(s)=\frac{55}{48 \sqrt{3}} \alpha_{0} \frac{\gamma^{5}}{|\rho(s)|^{3}}\left(\frac{\hbar}{m c}\right)^{2},
$$


with $\gamma$ the relativistic energy factor, $\rho(s)$ the bending radius of the bending magnets, $\hbar$ the reduced Planck constant, and $m$ the electron rest mass. As we see from the $\rho(s)$ in the denominator, diffusion only happens in the bending magnets.

Later in our applications, it will be convenient to make a transformation from $\vec{z}$ to the betatron coordinates $\vec{z}_{\beta}=\mathcal{B} \vec{z}$ where we defined the dispersion matrix $\mathcal{B}$ in Eq. (2.28). It follows that we will need to define

$$
D_{\beta}=\mathcal{B} D \mathcal{B}^{T}=d\left(\begin{array}{cccccc}
\eta_{x}^{2} & \eta_{x} \eta_{x}^{\prime} & \eta_{x} \eta_{y} & \eta_{x} \eta_{y}^{\prime} & 0 & -\eta_{x} \\
\eta_{x} \eta_{x}^{\prime} & \eta_{x}^{\prime 2} & \eta_{x}^{\prime} \eta_{y} & \eta_{x} \eta_{y}^{\prime} & 0 & -\eta_{x}^{\prime} \\
\eta_{x} \eta_{y} & \eta_{x}^{\prime} \eta_{y} & \eta_{y}^{2} & \eta_{y} \eta_{y}^{\prime} & 0 & -\eta_{y} \\
\eta_{x} \eta_{y}^{\prime} & \eta_{x}^{\prime} \eta_{y}^{\prime} & \eta_{y} \eta_{y}^{\prime} & \eta_{y}^{\prime 2} & 0 & -\eta_{y}^{\prime} \\
0 & 0 & 0 & 0 & 0 & 0 \\
-\eta_{x} & -\eta_{x}^{\prime} & -\eta_{y} & -\eta_{y}^{\prime} & 0 & 1
\end{array}\right)
$$

which we write in terms of $2 \times 2$ submatrices as

$$
D_{\beta}=\left(\begin{array}{ccc}
D_{x x} & D_{x y} & D_{x z} \\
D_{x y} & D_{y y} & D_{y z} \\
D_{z x} & D_{z y} & D_{z z}
\end{array}\right) .
$$

The uncoupled local diffusion coefficients are given by combining $G_{x, y, z}$ with $D_{\beta}$ :

$$
\begin{aligned}
& d_{x}=\operatorname{Tr}\left(G_{x} D_{\beta}\right)=d \mathcal{H}_{x}, \\
& d_{y}=\operatorname{Tr}\left(G_{y} D_{\beta}\right)=d \mathcal{H}_{y}, \\
& d_{z}=\operatorname{Tr}\left(G_{z} D_{\beta}\right)=d \beta_{z} \approx d \frac{a}{\mu_{s}} .
\end{aligned}
$$

We use the standard definition of $\mathcal{H}_{x, y}$

$$
\mathcal{H}_{x, y}=\gamma_{x, y} \eta_{x, y}^{2}+2 \alpha_{x, y} \eta_{x, y} \eta_{x, y}^{\prime}+\beta_{x, y}\left(\eta_{x, y}^{\prime}\right)^{2}
$$

The global diffusion coefficients $\bar{d}_{x}$ and $\bar{d}_{z}$ are given by integrating these quantities around the ring.

Damping occurs in the bending magnets and in the RF cavity. At an RF cavity, 
the particle gains back the average energy by the amount $U_{0}$, which it lost due to radiation one turn around the ring. It follows that the $\mathrm{RF}$ cavity contributes to a damping [36]

$$
x^{\prime} \rightarrow x^{\prime}\left(1-\frac{U_{0}}{c P_{0}}\right)
$$

which could be described as

$$
B_{\text {cav }}(s)=\left(\begin{array}{cccc}
0 & 0 & 0 & 0 \\
0 & \frac{U_{0}}{c P_{0}} \delta\left(s-s_{c}\right) & 0 & 0 \\
0 & 0 & 0 & 0 \\
0 & 0 & 0 & 0
\end{array}\right)
$$

where $s_{c}$ is the location of the cavity. In case of multiple RF cavities at positions $s_{c i}$, we define

$$
b_{x}(s)=\sum_{i} \frac{U_{0 i}}{c P_{0}} \delta\left(s-s_{c i}\right)
$$

with $\sum_{i} U_{0 i}=U_{0}$, i.e. the net energy loss is $U_{0}$.

For the damping effect due to the bending magnets, we have

$$
B_{\text {bend }}(s)=\left(\begin{array}{cccc}
0 & 0 & 0 & 0 \\
0 & 0 & 0 & 0 \\
0 & 0 & 0 & 0 \\
b_{\delta x} & 0 & 0 & b_{z}
\end{array}\right) \text {. }
$$

The $b_{z}$ term comes from the fact that a higher energy electron radiates more whereas the $b_{\delta x}$ term has two parts. The first comes from the fact that a particle with a larger value of $x$ takes a longer path through the magnet and the second from the fact that the power radiated depends on the magnetic field, which in turn depends on $x$ if the vertical magnetic field strength $B_{y}$ contains a quadrupole component. In separated function dipoles, this second part vanishes. The first is proportional to $\frac{1}{\rho}$. Specifically, we have

$$
b_{z}=P_{\gamma} c P_{0}, \quad b_{\delta x}=\frac{P_{\gamma}}{2 c E_{0}}\left(\frac{1}{\rho}+\frac{2}{B_{y}} \frac{\partial B_{y}}{\partial x}\right)
$$

where $c$ is the speed of light, $E_{0}=c P_{0}$ at high energy, is the reference energy of the 
particle, $P_{\gamma}$ is the instantaneous radiated power given by

$$
P_{\gamma}=\frac{e^{2} c^{3} C_{\gamma}}{2 \pi} E_{0}^{2} B_{y}^{2}
$$

$e$ is the electron charge, $r_{e}$ is the electron classical radius, and the constant

$$
C_{\gamma}=\frac{4 \pi}{3} \frac{r_{e}}{\left(m c^{2}\right)^{3}}=8.85 \times 10^{-5} \text { meter }-\mathrm{GeV}^{-3}
$$

$P_{\gamma}$ is only non-zero in the bending magnets. This is why we have written $B_{\text {bend }}$ in Eq.(4.40).

Altogether, then, we have

$$
B(s)=\left(\begin{array}{cccccc}
0 & 0 & 0 & 0 & 0 & 0 \\
0 & b_{x}(s) & 0 & 0 & 0 & 0 \\
0 & 0 & 0 & 0 & 0 & 0 \\
0 & 0 & 0 & b_{y}(s) & 0 & 0 \\
0 & 0 & 0 & 0 & 0 & 0 \\
b_{\delta x}(s) & 0 & 0 & 0 & 0 & b_{z}(s)
\end{array}\right)
$$

For later applications, we will again need to transform the damping matrix to the betatron coordinates. We then calculate

$$
\begin{aligned}
B_{\beta}= & \mathcal{B B B}^{-1} \\
= & \left(\begin{array}{cccccc}
-b_{\delta x} \eta_{x} & 0 & 0 & 0 & 0 & -b_{z} \eta_{x}-b_{\delta x} \eta_{x}^{2} \\
-b_{\delta x} \eta_{x}^{\prime} & b_{x} & 0 & 0 & 0 & \left(b_{x}-b_{z}\right) \eta_{x}^{\prime}-b_{\delta x} \eta_{x}^{\prime} \eta_{x} \\
-b_{\delta x} \eta_{y} & 0 & 0 & b_{y} & 0 & \left(b_{y}-b_{z}\right) \eta_{y}^{\prime}-b_{\delta x} \eta_{y}^{\prime} \eta_{x} \\
0 & -b_{x} \eta_{x} & 0 & -b_{y} \eta_{y} & 0 & -b_{x} \eta_{x}^{\prime} \eta_{x}-b_{y} \eta_{y}^{\prime} \eta_{y} \\
b_{\delta x} & 0 & 0 & 0 & 0 & b_{z}+b_{\delta x} \eta_{x}
\end{array}\right)
\end{aligned}
$$

Let us write this in terms of $2 \times 2$ submatrices as

$$
B_{\beta}=\left(\begin{array}{ccc}
B_{x x} & B_{x y} & B_{x z} \\
B_{x y} & B_{y y} & B_{y z} \\
B_{z x} & B_{y z} & B_{z z}
\end{array}\right) .
$$


To find the damping decrements, we use Eqs. (4.11) and (4.13). Using betatron coordinates, in the uncoupled case, the $U$ matrix and its inverse are block diagonal,

$$
U=\left(\begin{array}{cc}
U_{x} & 0 \\
0 & U_{z}
\end{array}\right), \quad U^{-1}=\left(\begin{array}{cc}
U_{x}^{-1} & 0 \\
0 & U_{z}^{-1}
\end{array}\right)
$$

where $U_{x, z}$ and $U_{x, z}^{-1}$ are $2 \times 2$ matrices. All these quantities depend on the position in the ring $s$. Eq.(4.13) then says

$$
\chi_{x}=\oint \operatorname{Tr}\left[U_{x}^{-1}(s) B(s) U_{x}(s)\right] d s=\oint \operatorname{Tr}\left[B_{x x}(s)\right] d s
$$

and likewise for $z$,

$$
\chi_{z}=\oint \operatorname{Tr}\left[B_{z z}\right] d s .
$$

Thus, in the uncoupled case, we find that

$$
\begin{aligned}
\chi_{x} & =\oint\left[b_{x}(s)-\eta b_{\delta x}(s)\right] d s \\
& \equiv \frac{U_{0}}{2 E_{0}}(1-\mathcal{D}) \equiv \frac{U_{0}}{2 E_{0}} \mathcal{J}_{x} \\
\chi_{z} & =\oint\left[b_{z}(s)+\eta b_{\delta x}(s)\right] d s \\
& \equiv \frac{U_{0}}{2 E_{0}}(2+\mathcal{D}) \equiv \frac{U_{0}}{2 E_{0}} \mathcal{J}_{z}
\end{aligned}
$$

where $U_{0}=\oint d s P_{\gamma}$ is the total radiated energy per turn and we have introduced the standard notation of $\mathcal{D}, \mathcal{J}_{x}$, and $\mathcal{J}_{z}$ for the damping partition numbers. This is a well-known result in [36], and a similar rederivation has been given in [32] and [33]. Note that one can think of dispersion as causing coupling, and that the local and global sum rules of Eqs.(4.18) and (4.19) are satisfied.

Now that we have the global diffusion coefficients and damping decrements for the uncoupled case, Eq. (4.10) gives the equilibrium values of the invariants, or in terms of the emittances $\epsilon_{a}=\left\langle g_{a}\right\rangle / 2$ we find

$$
\epsilon_{x}=\frac{\frac{55}{48 \sqrt{3}} \alpha_{0} \gamma^{5} \oint d s \frac{\mathcal{H}_{x}}{\left|\rho^{3}\right|}}{\frac{2 U_{0}}{E_{0}} \mathcal{J}_{x}}
$$




$$
\epsilon_{z}=\frac{\frac{55}{48 \sqrt{3}} \alpha_{0} \gamma^{5} \frac{a}{\mu_{s}} \oint d s \frac{1}{\left|\rho^{3}\right|}}{\frac{2 U_{0}}{E_{0}} \mathcal{J}_{z}}
$$




\section{Chapter 5}

\section{Coupling and Constant Damping/Diffusion Combined}

In the previous chapter, we saw how to find the equilibrium beam distribution in the presence of a constant diffusion and damping mechanism such as synchrotron radiation. We gave expressions for the general Gaussian solution along with expressions for the damping and diffusion coefficients for the eigen-invariants, to yield equilibrium emittances. We also gave the explicit results for the uncoupled case. In Chapter 2, we found expressions for the invariants near each of the linear resonances. In this chapter we would like to combine those results with Chapter 3, to find explicit expressions for the damping and diffusion coefficients of the eigen-invariants near linear resonances.

\subsection{Diffusion Coefficients}

From the invariants, we can find the local diffusion coefficients by right-multiplying by the diffusion matrix $D_{\beta}$ and taking the trace. 


\subsubsection{Sum/Difference Resonance}

The results for the sum/difference resonance are

$$
\begin{aligned}
& d_{1}^{+}=\cosh ^{2}\left(\frac{\theta}{2}\right) d_{x}+\sinh ^{2}\left(\frac{\theta}{2}\right) d_{z}+\sinh (\theta) d_{c}^{+}, \\
& d_{2}^{+}=\sinh ^{2}\left(\frac{\theta}{2}\right) d_{x}+\cosh ^{2}\left(\frac{\theta}{2}\right) d_{z}+\sinh (\theta) d_{c}^{+}, \\
& d_{1}^{-}=\cos ^{2}\left(\frac{\theta}{2}\right) d_{x}+\sin ^{2}\left(\frac{\theta}{2}\right) d_{z}+\sin (\theta) d_{c}^{-}, \\
& d_{2}^{-}=\sin ^{2}\left(\frac{\theta}{2}\right) d_{x}+\cos ^{2}\left(\frac{\theta}{2}\right) d_{z}-\sin (\theta) d_{c}^{-},
\end{aligned}
$$

with

$$
d_{c}^{ \pm}=\operatorname{Tr}\left[G_{c}^{ \pm} D_{\beta}\right]
$$

To get the global diffusion coefficient $\bar{d}_{a}$, we must integrate the local diffusion coefficients $d_{a}$ around the ring. For $d_{x}$ and $d_{z}$ this integration simply reproduces the global uncoupled diffusion coefficients $\bar{d}_{x}$ and $\bar{d}_{z}$. The extra term, $d_{c}$, which comes from the extra term in the invariants $G_{c}$, however, represents a new set of lattice functions to be integrated. It would be substantial complication if this full integration were required since it would require detailed knowledge of the lattice. Note however, that as with $G_{c}$, all of the terms in $d_{c}$ are proportional to $\cos \phi$ or $\sin \phi$. As we transform the $d_{a}$ around the ring, in addition to the lattice functions $\beta_{x, z}$ and $\eta_{x, z}$ evolving, the phase $\phi$ will also evolve as was discussed in Chapter 3.

The net change in $\phi$ around the ring is $\mu_{x}-\mu_{z}$ for the sum resonance and $\mu_{x}+\mu_{z}$ for the difference resonance. We can write this is as

$$
\Delta \phi=2 \pi n+\left[\mu_{x} \mp \mu_{z}\right]
$$

where the square brackets represent taking a modulus of $2 \pi$. Near resonance this term is small. For synchrobetaron coupling, typically $n$ is somewhat large, since $\nu_{z}$ is close to zero and $\nu_{x}$ is typically much greater than 1 . The result of this argument is that the middle term in the local diffusion coefficient $d_{c}$ will integrate to a small value 
in the typical situation, especially so if we assume some symmetry in the lattice. ${ }^{1}$ Thus, for our purposes here, we ignore this extra term in our global expressions, ${ }^{2}$

$$
\begin{aligned}
\bar{d}_{1}^{+} & =\cosh ^{2}\left(\frac{\theta}{2}\right) \bar{d}_{x}+\sinh ^{2}\left(\frac{\theta}{2}\right) \bar{d}_{z}, \\
\bar{d}_{2}^{+} & =\sinh ^{2}\left(\frac{\theta}{2}\right) \bar{d}_{x}+\cosh ^{2}\left(\frac{\theta}{2}\right) \bar{d}_{z}, \\
\bar{d}_{1}^{-} & =\cos ^{2}\left(\frac{\theta}{2}\right) \bar{d}_{x}+\sin ^{2}\left(\frac{\theta}{2}\right) \bar{d}_{z}, \\
\bar{d}_{2}^{-} & =\sin ^{2}\left(\frac{\theta}{2}\right) \bar{d}_{x}+\cos ^{2}\left(\frac{\theta}{2}\right) \bar{d}_{z} .
\end{aligned}
$$

\subsubsection{Integer/Half-integer Resonance}

We now consider the diffusion coefficients for the integer and half-integer resonances. For these resonances, we find

$$
d_{2}^{n}=d_{z} \cosh \theta+d_{c} \sinh \theta
$$

where

$$
d_{c}=\operatorname{Tr}\left(G_{c}^{n z} D_{z z}\right)
$$

with the results for $x$ given by replacing $z$ with $x$ in these expressions.

We must now integrate the local diffusion coefficient around the ring to get the global diffusion coefficient. For the case of $x$, applying the same argument as in the sum and difference resonances, we find that the extra term $d_{c}$ integrates to a small value and we disregard it. For the longitudinal direction, however, there is no change in $\phi$ around the ring, and thus we must keep $d_{c}$, with its initial value. Thus, for the

\footnotetext{
${ }^{1}$ In the case of $x-y$ betatron coupling in which $\nu_{x} \approx \nu_{y}$ is possible, this argument will need to be revised.

${ }^{2}$ We note here that in case one wanted to compute these middle terms exactly, then, $\cos \phi$ and $\sin \phi$ can be expanded in terms of $\cos \phi\left(s_{1}\right), \sin \phi\left(s_{1}\right)$ and cosine and sine of the phase advances $\psi_{x 12}$ and $\psi_{z 12}$. We could then write out the expressions $b_{c}$ and $d_{c}$ explicitly in terms of unperturbed lattice functions. Then, for a given lattice, one could compute these integrals once and for all, and not have to recompute different integrals, depending on the form of the coupling, one would simply take different linear combinations of these integrals depending on the value of $\phi\left(s_{1}\right)$.
} 
global diffusion coefficients, we take

$$
\bar{d}_{1}=\cosh (\theta) \bar{d}_{x}
$$

It may also be useful to apply Eq. (3.51) or Eq. (3.52) for the integer or half integer resonances to express in terms of $\mu_{1}$ and $\Delta \mu$. This is for near the integer or half integer $x$ resonance and

$$
\bar{d}_{2}=(\cosh \theta+\cos \phi \sinh \theta) \bar{d}_{z}
$$

near the integer $z$ resonance. For the $x$ resonances, the $\bar{d}_{2}$ is not strongly affected, while for the $z$ resonance, $\bar{d}_{1}$ is not strongly affected and we assume there is no change in these quantities.

\subsection{Damping Coefficients}

Next we find the local damping coefficients. We do this by constructing the matrix $A=U^{-1} B_{\beta} U\left[B_{\beta}\right.$ is defined in Eq.(4.45)] with $U$ constructed from the perturbed eigenvectors for each resonance. From $A$ we find $b_{a}=A_{a a}+A_{-a-a}$.

\subsubsection{Sum/Difference Resonance}

The damping coefficients for the sum and difference resonances are given by

$$
\begin{aligned}
b_{1}^{+} & =b_{x \beta} \cosh ^{2}\left(\frac{\theta}{2}\right)-b_{z \beta} \sinh ^{2}\left(\frac{\theta}{2}\right)+\sinh (\theta) b_{c}^{+}, \\
b_{2}^{+} & =-b_{x \beta} \sinh ^{2}\left(\frac{\theta}{2}\right)+b_{z \beta} \cosh ^{2}\left(\frac{\theta}{2}\right)-\sinh (\theta) b_{c}^{+}, \\
b_{1}^{-} & =b_{x \beta} \cos ^{2}\left(\frac{\theta}{2}\right)+b_{z \beta} \sin ^{2}\left(\frac{\theta}{2}\right)+\sin (\theta) b_{c}^{-}, \\
b_{2}^{-} & =b_{x \beta} \sin ^{2}\left(\frac{\theta}{2}\right)+b_{z \beta} \cos ^{2}\left(\frac{\theta}{2}\right)-\sin (\theta) b_{c}^{-},
\end{aligned}
$$


with

$$
\begin{aligned}
& b_{x \beta}=\operatorname{Tr}\left[B_{x x}\right]=b_{x}-\eta b_{\delta x}, \\
& b_{z \beta}=\operatorname{Tr}\left[B_{z z}\right]=b_{z}+\eta b_{\delta x},
\end{aligned}
$$

where

$$
\begin{aligned}
b_{c}^{+}= & \frac{1}{2 \sqrt{\beta_{x} \beta_{z}}}\left[\left(-\left(b_{x}-b_{z}+b_{\delta x} \eta\right)\left(\left(\alpha_{x}+\alpha_{z}\right) \eta+\beta_{x} \eta^{\prime}\right)\right) \cos \phi\right. \\
& \left.+\left(\left(b_{x}-b_{z}\right)\left(-\eta+\alpha_{x} \alpha_{z} \eta+\alpha_{z} \beta_{x} \eta^{\prime}\right)+b_{\delta x}\left(\beta_{x} \beta_{z}+\left(1-\alpha_{x} \alpha_{z}\right) \eta^{2}-\alpha_{z} \beta_{x} \eta \eta^{\prime}\right)\right) \sin \phi\right],(5.10) \\
b_{c}^{-}= & \frac{1}{2 \sqrt{\beta_{x} \beta_{z}}}\left[\left(b_{x}-b_{z}\right)\left(\left(1+\alpha_{x} \alpha_{z}\right) \eta+\alpha_{z} \beta_{x} \eta^{\prime}\right)-b_{\delta x}\left(-\beta_{x} \beta_{z}+\eta^{2}+\alpha_{x} \alpha_{z} \eta^{2}+\alpha_{z} \beta_{x} \eta \eta^{\prime}\right) \cos \phi\right. \\
& -\left(b_{x}-b_{z}-b_{\delta x} \eta\left[\left(\left(\alpha_{x}-\alpha_{z}\right) \eta+\beta_{x} \eta^{\prime}\right] \sin \phi\right] .\right.
\end{aligned}
$$

Again, as with the local diffusion coefficients, we find an extra term in $b_{a}$, in addition to the uncoupled damping coefficients $b_{x \beta}$ and $b_{z \beta}$. Using the same argument as for the diffusion coefficients, we find that this quantity integrates to a small value and we will discard it in our global expressions. In addition, note that $b_{c}^{ \pm}$is proportional to either $1 / \beta_{z}$ or $b_{\delta x}$. For the case of synchrobetatron coupling, both $1 / \beta_{z}$ and $b_{\delta x}$ are small, and so this term is intrinsically small. The integration of $b_{x \beta}$ and $b_{z \beta}$ give the global uncoupled damping decrements, $\chi_{x}$ and $\chi_{z}$. Thus, for our global coupled damping coefficients, we take

$$
\begin{aligned}
& \chi_{1}^{+}=\chi_{x} \cosh ^{2}\left(\frac{\theta}{2}\right)-\chi_{z} \sinh ^{2}\left(\frac{\theta}{2}\right), \\
& \chi_{2}^{+}=-\chi_{x} \sinh ^{2}\left(\frac{\theta}{2}\right)+\chi_{z} \cosh ^{2}\left(\frac{\theta}{2}\right), \\
& \chi_{1}^{-}=\chi_{x} \cos ^{2}\left(\frac{\theta}{2}\right)+\chi_{z} \sin ^{2}\left(\frac{\theta}{2}\right), \\
& \chi_{2}^{-}=\chi_{x} \sin ^{2}\left(\frac{\theta}{2}\right)+\chi_{z} \cos ^{2}\left(\frac{\theta}{2}\right) .
\end{aligned}
$$

The damping decrements for the sum resonance show an interesting effect. One of $\chi_{1,2}$ will become negative for a finite value of $\theta$. Specifically, suppose that $\chi_{z}>\chi_{x}$ 
which is typically the case. Then $\chi_{1}$ vanishes when

$$
\sqrt{\frac{\chi_{z}}{\chi_{x}}}=\operatorname{coth}\left(\frac{\theta}{2}\right)
$$

For $\theta$ larger than this, $\chi_{1}$ becomes negative, and there is an instability. This is analogous to the case where the damping partition number $\mathcal{D}$ is greater than 1 , in which case, we can see from Eq.(4.50) that $\chi_{x}$ is likewise negative, indicating an instability. We refer to this as an "anti-damping instability" in this thesis.

\subsubsection{Integer/Half-integer Resonances}

For the integer/half-integer resonance, we find that the local damping coefficients are unchanged. This is clear because the perturbation only changes the $U$ matrix in either the upper left or lower right $2 \times 2$ block, depending on which resonance is being considered. But this reduces the problem to the 1-D case. Consider the $\nu_{z}$ integer or half-integer resonance. The damping coefficient for mode 2 is given by

$$
b_{2}=\operatorname{Tr}\left(U_{2}^{-1} B_{z z} U_{2}\right)=\operatorname{Tr}\left(B_{z z}\right)=b_{z \beta},
$$

where $U_{2}$ is the submatrix of $U$ built out of $\tilde{v}_{20}$ and $\tilde{v}_{-20}$. We have used the cyclic property of the trace. In other words, the damping decrements are unchanged to lowest order near the integer/half integer resonances.

\subsection{Equilibrium Eigen-Emittances}

We summarize the results for the diffusion and damping in Tables 5.1 and 5.2. The reader can verify that all the quantities satisfy the sum rules discussed in Section 4.1.

From the global quantities $\bar{d}_{1,2}$ and $\chi_{1,2}$, we find the equilibrium average values of the invariants from Eq.(4.10). We quote RMS eigen-emittances, which are $1 / 2$ the value of the average eigen-invariants. 
For the sum resonance, we find

$$
\begin{aligned}
\epsilon_{1}^{+} & =\frac{\cosh ^{2} \frac{\theta}{2} \bar{d}_{x}+\sinh ^{2} \frac{\theta}{2} \bar{d}_{z}}{4\left(\cosh ^{2} \frac{\theta}{2} \chi_{x}-\sinh ^{2} \frac{\theta}{2} \chi_{z}\right)} \\
\epsilon_{2}^{+} & =\frac{\sinh ^{2} \frac{\theta}{2} \bar{d}_{x}+\cosh ^{2} \frac{\theta}{2} \bar{d}_{z}}{4\left(-\sinh ^{2} \frac{\theta}{2} \chi_{x}+\cosh ^{2} \frac{\theta}{2} \chi_{z}\right)}
\end{aligned}
$$

while for the difference resonance, we find

$$
\begin{aligned}
\epsilon_{1}^{-} & =\frac{\cos ^{2} \frac{\theta}{2} \bar{d}_{x}+\sin ^{2} \frac{\theta}{2} \bar{d}_{z}}{4\left(\cos ^{2} \frac{\theta}{2} \chi_{x}+\sin ^{2} \frac{\theta}{2} \chi_{z}\right)} \\
\epsilon_{2}^{-} & =\frac{\sin ^{2} \frac{\theta}{2} \bar{d}_{x}+\cos ^{2} \frac{\theta}{2} \bar{d}_{z}}{4\left(\sin ^{2} \frac{\theta}{2} \chi_{x}+\cos ^{2} \frac{\theta}{2} \chi_{z}\right)}
\end{aligned}
$$

Note that in the case where $\chi_{x}=\chi_{z}$, we find that

$$
\begin{aligned}
\epsilon_{1}^{+} & =\cosh ^{2} \frac{\theta}{2} \epsilon_{x}+\sinh ^{2} \frac{\theta}{2} \epsilon_{z} \\
\epsilon_{2}^{+} & =\sinh ^{2} \frac{\theta}{2} \epsilon_{x}+\cosh ^{2} \frac{\theta}{2} \epsilon_{z}
\end{aligned}
$$

for the sum resonance and

$$
\begin{aligned}
\epsilon_{1}^{-} & =\cos ^{2} \frac{\theta}{2} \epsilon_{x}+\sin ^{2} \frac{\theta}{2} \epsilon_{z} \\
\epsilon_{2}^{-} & =\sin ^{2} \frac{\theta}{2} \epsilon_{x}+\cos ^{2} \frac{\theta}{2} \epsilon_{z}
\end{aligned}
$$

for the difference resonance. Thus, in this case, it makes sense to talk about emittance coupling: the effect of the coupling is simply to mix together the equilibrium emittances. If we were talking about transverse $x-y$ coupling, $\chi_{x}=\chi_{z}$ would indeed be approximately correct in many situations and this gives a justification for using that concept for betatron coupling. For the case here of synchrobetatron coupling, typically $\chi_{x} \approx \chi_{z} / 2$, and thus the concept of emittance coupling is not precise.

For the integer and half integer resonances, we saw in Eq. (5.14) that the damping partition number is not affected by the coupling to lowest order. The global diffusion coefficient is affected, however. In fact it diverges at the resonance. For the integer 
and half integer $x$ resonances, we find an emittance growth of

$$
\frac{\epsilon_{1}}{\epsilon_{x}}=\frac{\bar{d}_{1}}{\bar{d}_{x}}=\cosh \theta
$$

with $\theta$ the appropriate coupling angle. $\epsilon_{z}$ is not strongly affected, and we take $\epsilon_{2}=\epsilon_{z}$. As noted in Eq. (5.6), one could also write this as

$$
\frac{\epsilon_{1}}{\epsilon_{x}}=\frac{\Delta \mu}{2 \mu_{1}}
$$

for the integer resonance, or

$$
\frac{\epsilon_{1}}{\epsilon_{x}}=\frac{\Delta \mu}{2\left(\mu_{1}-\pi\right)}
$$

for the half integer resonance.

Now, because $\mu_{z}$ is typically small, we only have an integer resonance. As discussed, for this case, the $\cos \phi$ term doesn't average away, and we are left with

$$
\frac{\epsilon_{2}}{\epsilon_{z}}=\cosh \theta+\cos \phi \sinh \theta
$$

$\epsilon_{x}$ is not much affected here, and we take $\epsilon_{1}=\epsilon_{x}$. If there were a half integer $z$ resonance, or indeed, another integer resonance where $\mu_{z}$ is near an integer other than 0 , the second term in this equation would integrate to a small value.

\subsection{Sigma Matrices}

In the previous subsection, we gave the expressions for the equilibrium eigen-emittances near each of the resonances. One may also wish to know the projected emittances which are more closely related to the typically measured quantities for a beam. Finally, we give expressions for the second moments of the beam distribution at equilibrium. For the sum and difference resonances, using the invariants and Eq.(2.27), we can derive

$$
\Sigma^{+}=\left(\begin{array}{cc}
\epsilon_{x, \mathrm{pr}}^{+} \Sigma_{x} & \Sigma_{c}^{+} \\
\Sigma_{c}^{+T} & \epsilon_{z, \mathrm{pr}}^{+} \Sigma_{z}
\end{array}\right),
$$




\begin{tabular}{|lcccc|}
\hline resonance & mode & $d$ & $d_{c}$ & $d$ \\
\hline \hline sum & 1 & $d_{x} \cosh ^{2} \frac{\theta}{2}+d_{z} \sinh ^{2} \frac{\theta}{2}+d_{c} \sinh \theta$ & & $\cosh ^{2}\left(\frac{\theta}{2}\right) d_{x}+\sinh ^{2}\left(\frac{\theta}{2}\right) d_{z}$ \\
& 2 & $d_{x} \sinh ^{2} \frac{\theta}{2}+d_{z} \cosh ^{2} \frac{\theta}{2}+d_{c} \sinh \theta$ & $\operatorname{Tr}\left[G_{c}^{+} D_{\beta}\right]$ & $\sinh ^{2}\left(\frac{\theta}{2}\right) \bar{d}_{x}+\cosh ^{2}\left(\frac{\theta}{2}\right) \bar{d}_{z}$ \\
\hline difference & 1 & $d_{x} \cos ^{2} \frac{\theta}{2}+d_{z} \sin ^{2} \frac{\theta}{2}+d_{c} \sin \theta$ & & $\cos ^{2}\left(\frac{\theta}{2}\right) d_{x}+\sin ^{2}\left(\frac{\theta}{2}\right) d_{z}$ \\
& 2 & $d_{x} \sin ^{2} \frac{\theta}{2}+d_{z} \cos ^{2} \frac{\theta}{2}-d_{c} \sin \theta$ & $\operatorname{Tr}\left[G_{c}^{-} D_{\beta}\right]$ & $\sin ^{2}\left(\frac{\theta}{2}\right) \bar{d}_{x}+\cos ^{2}\left(\frac{\theta}{2}\right) \bar{d}_{z}$ \\
\hline int/ $\frac{1}{2}$-int $(x)$ & 1 & $d_{x} \cosh \theta+d_{c} \sinh \theta$ & $d_{x} \cosh \theta$ \\
& 2 & $d_{z}$ & $\operatorname{Tr}\left[G_{c}^{n x} D_{\beta}\right]$ & $\bar{d}_{z}$ \\
\hline int $(z)$ & 1 & $d_{x}$ & & $d_{x}$ \\
& 2 & $d_{z} \cosh \theta+d_{c} \sinh \theta$ & $\operatorname{Tr}\left[G_{c}^{n z} D_{\beta}\right]$ & $\bar{d}_{z}(\cosh \theta+\cos \phi \sinh \theta)$ \\
\hline
\end{tabular}

Table 5.1: This table contains the diffusion coefficients near each of the linear resonances. The $d$ column gives the local diffusion coefficient for modes 1 and 2 . The $d_{c}$ column gives the formula for the extra term $d_{c}$ contained in the corresponding local diffusion coefficient. Finally, the $\bar{d}$ column contains the global diffusion coefficient which is given by integrating the local quantity. The text discusses the approximations used for these expressions. The angle $\theta$ is given by $\tan ^{-1}(\xi / \Delta \mu)$ for the difference resonance and $\tanh ^{-1}(\xi / \Delta \mu)$ for the other resonances where $\xi$ and $\Delta \mu$ are given in table 3.1. The phase $\phi$ for the integer $z$ resonance is also given in table 3.1.

\begin{tabular}{|lcccc|}
\hline resonance & mode & $b$ & $b_{c}$ & $\chi$ \\
\hline \hline sum & 1 & $b_{x \beta} \cosh ^{2} \frac{\theta}{2}-b_{z \beta} \sinh ^{2} \frac{\theta}{2}+b_{c} \sinh \theta$ & & $\chi_{x} \cosh ^{2}\left(\frac{\theta}{2}\right)-\chi_{z} \sinh ^{2}\left(\frac{\theta}{2}\right)$ \\
& 2 & $-b_{x \beta} \sinh ^{2} \frac{\theta}{2}+b_{z \beta} \cosh ^{2} \frac{\theta}{2}-b_{c} \sinh \theta$ & Eq. $(5.10)$ & $-\chi_{x} \sinh ^{2}\left(\frac{\theta}{2}\right)+\chi_{z} \cosh ^{2}\left(\frac{\theta}{2}\right)$ \\
\hline difference & 1 & $b_{x \beta} \cos ^{2} \frac{\theta}{2}+b_{z \beta} \sin ^{2} \frac{\theta}{2}+b_{c} \sin \theta$ & & $\chi_{x} \cos ^{2}\left(\frac{\theta}{2}\right)+\chi_{z} \sin ^{2}\left(\frac{\theta}{2}\right)$ \\
& 2 & $b_{x \beta} \sin ^{2} \frac{\theta}{2}+b_{z \beta} \cos ^{2} \frac{\theta}{2}-b_{c} \sin \theta$ & Eq. $(5.11)$ & $\chi_{x} \sin ^{2}\left(\frac{\theta}{2}\right)+\chi_{z} \cos ^{2}\left(\frac{\theta}{2}\right)$ \\
\hline int/ $\frac{1}{2}$-int & 1 & $b_{x \beta}$ & $\chi_{x}$ \\
& 2 & $b_{z \beta}$ & $\chi_{z}$ \\
\hline
\end{tabular}

Table 5.2: This table contains the damping coefficients near each of the linear resonances. The $b$ column gives the local damping coefficient for modes 1 and 2 . The $b_{c}$ column tells where to find the formula for the extra term $b_{c}$ contained in the corresponding local damping coefficient. Finally, the $\chi$ column contains the global damping decrement which is given by integrating the local quantity. The approximations used for these expressions are discussed in the text. The angle $\theta$ is given by $\tan ^{-1}(\xi / \Delta \mu)$ for the difference resonance and $\tanh ^{-1}(\xi / \Delta \mu)$ for the other resonances where $\xi$ and $\Delta \mu$ are given in table 3.1. 
where

$$
\begin{aligned}
\epsilon_{x, \mathrm{pr}}^{+} & =\frac{1}{2}\left\langle g_{1}\right\rangle_{\mathrm{eq}}^{+} \cosh ^{2}\left(\frac{\theta}{2}\right)+\frac{1}{2}\left\langle g_{2}\right\rangle_{\mathrm{eq}}^{+} \sinh ^{2}\left(\frac{\theta}{2}\right), \\
\epsilon_{z, \mathrm{pr}}^{+} & =\frac{1}{2}\left\langle g_{1}\right\rangle_{\mathrm{eq}}^{+} \sinh ^{2}\left(\frac{\theta}{2}\right)+\frac{1}{2}\left\langle g_{2}\right\rangle_{\mathrm{eq}}^{+} \cosh ^{2}\left(\frac{\theta}{2}\right),
\end{aligned}
$$

and

$$
\Sigma^{-}=\left(\begin{array}{cc}
\epsilon_{x, \mathrm{pr}}^{-} \Sigma_{x} & \Sigma_{c}^{-} \\
\Sigma_{c}^{-T} & \epsilon_{z, \mathrm{pr}}^{-} \Sigma_{z}
\end{array}\right)
$$

where

$$
\begin{aligned}
\epsilon_{x, \mathrm{pr}}^{-} & =\frac{1}{2}\left\langle g_{1}\right\rangle_{\mathrm{eq}}^{-} \cos ^{2}\left(\frac{\theta}{2}\right)+\frac{1}{2}\left\langle g_{2}\right\rangle_{\mathrm{eq}}^{-} \sin ^{2}\left(\frac{\theta}{2}\right), \\
\epsilon_{z, \mathrm{pr}}^{-} & =\frac{1}{2}\left\langle g_{1}\right\rangle_{\mathrm{eq}}^{-} \sin ^{2}\left(\frac{\theta}{2}\right)+\frac{1}{2}\left\langle g_{2}\right\rangle_{\mathrm{eq}}^{-} \cos ^{2}\left(\frac{\theta}{2}\right),
\end{aligned}
$$

with

$\Sigma_{c}^{-}=\frac{-1}{8}\left[\left\langle g_{1}\right\rangle_{\mathrm{eq}}^{-}-\left\langle g_{2}\right\rangle_{\mathrm{eq}}^{-}\right] \sin (\theta)\left(\begin{array}{cc}-\sqrt{\beta_{x} \beta_{z}} \sin \phi & \sqrt{\frac{\beta_{x}}{\beta_{z}}}\left(\cos \phi+\alpha_{z} \sin \phi\right) \\ -\sqrt{\frac{\beta_{z}}{\beta_{x}}}\left(\cos \phi-\alpha_{x} \sin \phi\right) & -\frac{\left(\alpha_{x}-\alpha_{z}\right) \cos \phi+\left(1+\alpha_{x} \alpha_{z}\right) \sin \phi}{\sqrt{\beta_{x} \beta_{z}}}\end{array}\right)$,

and

$\Sigma_{c}^{+}=-\frac{1}{8}\left[\left\langle g_{1}\right\rangle_{\mathrm{eq}}^{+}+\left\langle g_{2}\right\rangle_{\mathrm{eq}}^{+}\right] \sinh (\theta)\left(\begin{array}{cc}\sqrt{\beta_{x} \beta_{z}} \cos \phi & -\sqrt{\frac{\beta_{x}}{\beta_{z}}}\left(\alpha_{z} \cos \phi+\sin \phi\right) \\ -\sqrt{\frac{\beta_{z}}{\beta_{x}}}\left(\alpha_{x} \cos \phi+\sin \phi\right) & \frac{\left(-1+\alpha_{x} \alpha_{z}\right) \cos \phi+\left(\alpha_{x}+\alpha_{z}\right) \sin \phi}{\sqrt{\beta_{x} \beta_{z}}}(5.34)\end{array}\right)$.

The uncoupled $2 \times 2$ matrices $\Sigma_{x, z}$ are given by

$$
\Sigma_{x, z}=\left(\begin{array}{cc}
\beta_{x, z} & \alpha_{x, z} \\
\alpha_{x, z} & \gamma_{x, z}
\end{array}\right)
$$

$\epsilon_{x(, z), \mathrm{pr}}$ are the projected horizontal and longitudinal emittances. The transverse beam size and momenta are given by

$$
\begin{aligned}
& \left\langle x_{\beta}^{2}\right\rangle=\epsilon_{x, \mathrm{pr}} \beta_{x}, \\
& \left\langle x_{\beta}^{\prime 2}\right\rangle=\epsilon_{x, \mathrm{pr}} \gamma_{x},
\end{aligned}
$$


and likewise for the bunch length and energy spread. Note however that these are in betatron coordinates and so differ from the physical variables by terms involving the dispersion.

\subsubsection{Beam Profile Near Difference Resonance}

For the difference resonance, the mixed spatial second moment is given by

$$
\left\langle x_{\beta} z_{\beta}\right\rangle=-\frac{1}{4}\left(\epsilon_{x}-\epsilon_{y}\right) \sin \theta \sqrt{\beta_{x} \beta_{y}} \cos \phi
$$

The spatial distribution of the beam will be a 2-D Gaussian with elliptical contours of constant density. The rotation angle of those ellipses is given by

$$
\tan \left(2 \theta_{r}\right)=\frac{2\left\langle x_{\beta} z_{\beta}\right\rangle}{\left\langle x_{\beta}^{2}\right\rangle-\left\langle z_{\beta}^{2}\right\rangle}
$$

In the case that $\beta_{x}=\beta_{z}$, we find that

$$
\tan \left(2 \theta_{r}\right)=\tan (\theta) \cos \phi
$$

We have been using $x-z$ synchrobetatron space in our analysis, but of course, the exact same analysis applies to transverse betatron coupling where $z$ is replaced by $y$. In this case, the difference resonance, is of common practical interest, and in fact many rings are operated right on this coupling resonance. In the case that the integer parts of the degenerate tunes aren't equal, $\phi$ will vary around the ring. Eq. (5.40) then says that the rotation angle of the beam distribution will also vary around the ring, with a maximum value of half the global coupling angle $\theta$.

The maximum global coupling angle occurs when $\Delta \mu \rightarrow 0$. In this case, we get $\theta= \pm \pi / 2$ with the sign determined by the direction in which 0 is approached. Thus, the rotation angle $\theta_{r}$ will reach a maximum value of $\theta=\pi / 4$ or $45^{\circ}$ The eigenemittances are both equal to

$$
\epsilon_{1,2}^{-}=\frac{\bar{d}_{x}+\bar{d}_{z}}{4\left(\chi_{x}+\chi_{z}\right)}
$$

and are also equal to the projected emittances. Thus, the beam contours in the 
$x_{\beta}-z_{\beta}$ space are round. For the corresponding transverse case, this is the desired effect. Thus, operating on the difference resonance is a relatively easy way to produce round beams.

One additional point about the difference resonance is that the difference in the eigen-phase advance exactly on resonance $(\Delta \mu=0)$ gives the global coupling parameter $\xi$. Varying the strength of a quadrupole magnet in the ring increases one of the tunes and decreases the other, thus changing $\Delta \mu$. Thus, by varying the strength of a quadrupole magnet near the difference resonance, and measuring the resulting eigen-tunes, the coupling parameter can be measured. This situation is illustrated in figure 5.1 in which the perturbed and unperturbed phase advances are plotted with varying $\Delta \mu$, along with the corresponding beam distribution at several values of $\Delta \mu$.

\subsubsection{Projected Emittance Sum Rules}

We have thus derived how these quantities are affected by coupling. We can now find some useful relationships among these quantities. First we have the obvious ones

$$
\epsilon_{x, \mathrm{pr}}^{ \pm} \mp \epsilon_{z, \mathrm{pr}}^{ \pm}=\left\langle g_{x}\right\rangle_{\mathrm{eq}}^{ \pm} \mp\left\langle g_{z}\right\rangle_{\mathrm{eq}}^{ \pm}
$$

We can also show that

$$
\chi_{x} \epsilon_{x, \mathrm{pr}}^{-}+\chi_{z} \epsilon_{z, \mathrm{pr}}^{-}=\chi_{x} \epsilon_{x}+\chi_{z} \epsilon_{z}
$$

and

$$
\chi_{x} \epsilon_{x, \mathrm{pr}}^{+}-\chi_{z} \epsilon_{z, \mathrm{pr}}^{+}=\chi_{x} \epsilon_{x}-\chi_{z} \epsilon_{z}
$$

Here, $\epsilon_{x, z}$ are the uncoupled emittances. These last two equations are only strictly true if the middle term in the global damping decrements is zero. However, this term will often be small due to the oscillatory nature of the integrand as we have discussed previously. Compare these sum rules to the sum rules for the equilibrium emittances of the eigenmodes near a sum/difference resonance (Eqs. (4.22)), one simply replaces $\chi_{1,2}$ with $\chi_{x, z}$ and $\left\langle g_{1,2}\right\rangle$ with $\epsilon_{x, z, p r}$.

Finally, consider the equilibrium $\Sigma$ matrix near an integer/half integer resonance. 


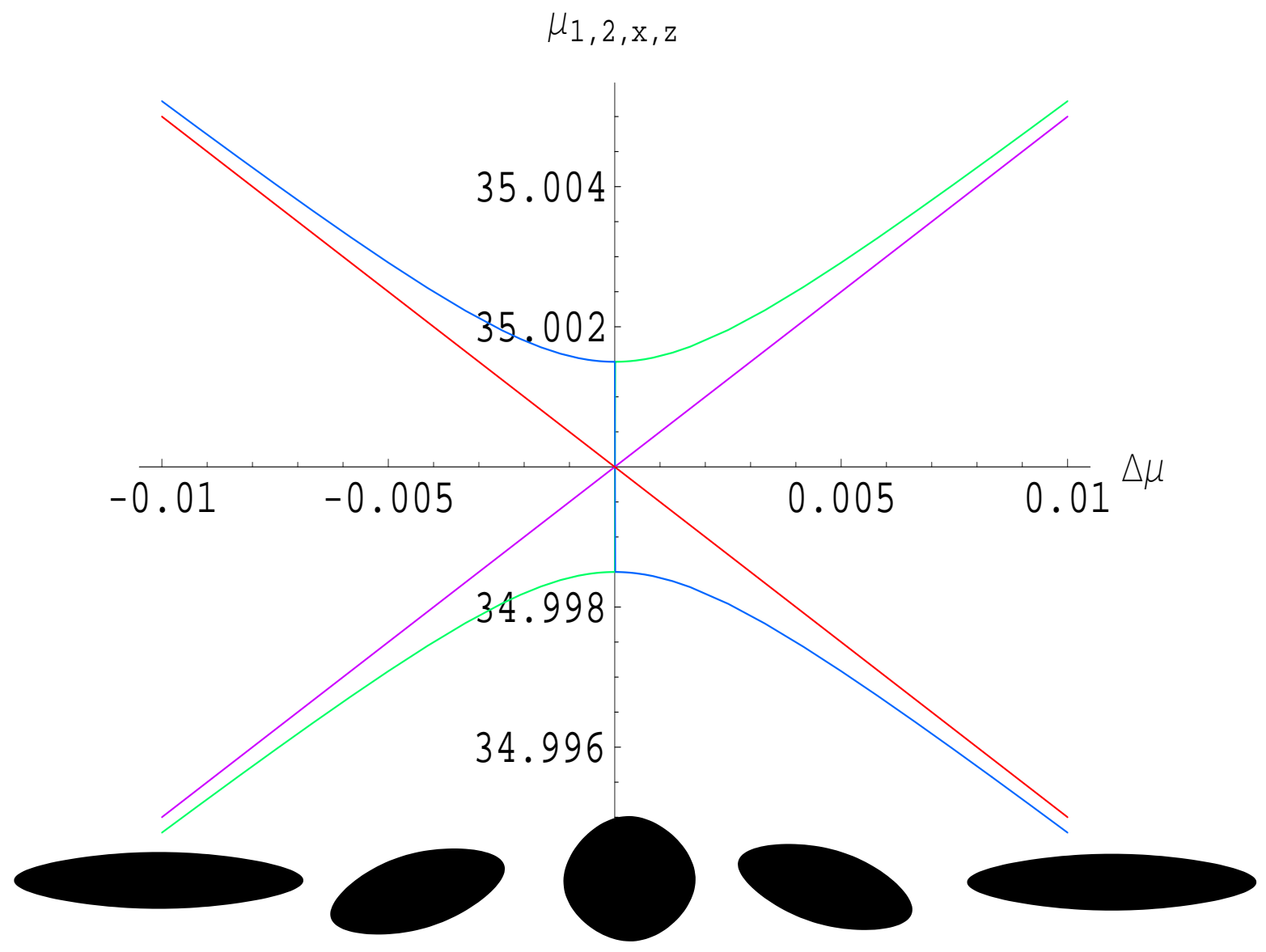

Figure 5.1: Here we plot the perturbed and unperturbed tunes with the addition of coupling as the unperturbed tune split is varied. For the case of transverse coupling, the tune split can be varied by changing the strength of a quadrupole magnet. The ellipses show contours of the beam distribution in $x_{\beta}-z_{\beta}$ space. The outer two ellipses represent the beam relatively far from the coupling resonance. The second and fourth ellipses represent the beam at approximately $\theta=45^{\circ}$, while the center round beam is exactly on the coupling resonance at $\theta=90^{\circ}$. The unperturbed phase advances $\mu_{x}$ and $\mu_{z}$ are purple and red respectively. With coupling, $\mu_{x}$ and $\mu_{z}$ become $\mu_{1}$ and $\mu_{2}$ which are represented by the green and blue curves respectively. The global coupling parameter is $\xi=0.003$ which can be read from the difference between $\mu_{1}$ and $\mu_{2}$ exactly on resonance. Note that the identity of $\mu_{1}$ and $\mu_{2}$ switch discontinuously with respect to the continuous curves in the upper and lower regions of the diagram. We have assumed that we are at a position in the ring where $\phi=0$. For different values of $\phi$, the second and fourth of the beam distributions would be rotated at different angles. 
As noted with Eq. (3.101), one can think of the effect of the coupling as causing a perturbation to the lattice parameters. Thus, in computing $\Sigma$, one must take two effects into account: the perturbation to the lattice parameters, and the perturbation to the diffusion coefficient. The perturbed diffusion coefficients are given in Table IV, and the rest of the perturbed lattice parameters can be found in the same way as in the derivation of Eq. (3.101).

\subsection{Applications}

We now apply these results to the applications considered in Chapter 2. We consider a storage ring with parameters drawn from the PEP-II Low Energy Ring which we list in Table 5.3.

\subsection{Global Quantities Near Sum/Difference Reso- nances}

For the sum and difference resonances, in Figures 5.2 and 5.3, we plot the damping decrements $\chi_{1,2}$, the coupled integrated diffusion coefficients $\bar{d}_{1,2}$ and the resulting equilibrium invariants $\left\langle g_{1,2}\right\rangle_{\mathrm{eq}}$. In Figures 5.4 and 5.5, we plot the projected emittances, $\epsilon_{x, z \text {,pr }}$ which are given by Eqs.(5.28), (5.29), (5.31), and (5.32). From these quantities, one can determine the equilibrium transverse beam size and bunch length as given in Eqs.(5.36) and (5.37). In Figures 5.6 and 5.7 we again plot the projected emittances from Figures 5.4 and 5.5, but with 3-D plots showing the full scale range.

\subsection{Anti-damping Instability}

An interesting effect that we have seen is that the damping decrement can become negative near a sum resonance when the motion is otherwise stable. This region of anti-damping is larger, the larger the ratio of $\chi_{z}$ and $\chi_{x}$, see Eq.(5.13). For the parameters used here, it is quite a small region on the $\nu_{x}-\nu_{z}$ plot for both of our 
Table 5.3: Parameters used in our numerical examples based on the PEP-II Low Energy Storage Ring[59].

\begin{tabular}{|cc|}
\hline parameter & value \\
\hline \hline $\mathrm{C}$ & $2199.33 \mathrm{~m}$ \\
\hline$\alpha_{c}$ & $1.23 \times 10^{-3}$ \\
\hline$\chi_{x}$ & $1.19 \times 10^{-4}$ \\
\hline$\chi_{z}$ & $2.4 \times 10^{-4}$ \\
\hline$\epsilon_{x}$ & $49.2 \times 10^{-9} \mathrm{~m}$ \\
\hline$\epsilon_{z}$ & $9.35 \times 10^{-6} \mathrm{~m}$ \\
\hline$d_{x}$ & $2.34 \times 10^{-11} \mathrm{~m}$ \\
\hline$d_{z}$ & $8.98 \times 10^{-9} \mathrm{~m}$ \\
\hline$\beta\left(s_{\text {cav }}\right)$ & $20 \mathrm{~m}$ \\
\hline$\alpha\left(s_{\text {cav }}\right)$ & 0 \\
\hline$\beta\left(s_{\text {crab }}\right)$ & $20 \mathrm{~m}$ \\
\hline$\alpha\left(s_{\text {crab }}\right)$ & 0 \\
\hline$\eta\left(s_{\text {cav }}\right)$ & $0-3 \mathrm{~m}$ \\
\hline$\eta^{\prime}\left(s_{\text {cav }}\right)$ & 0 \\
\hline$\eta\left(s_{\text {crab }}\right)$ & $0-3 \mathrm{~m}$ \\
\hline$\eta^{\prime}\left(s_{\text {crab }}\right)$ & 0 \\
\hline$\xi_{c}$ & $0-.0031 / \mathrm{m}$ \\
\hline$\nu_{x}$ & 36.56 \\
\hline$\nu_{y}$ & 38.51 \\
\hline$\nu_{s}$ & 0.025 \\
\hline
\end{tabular}



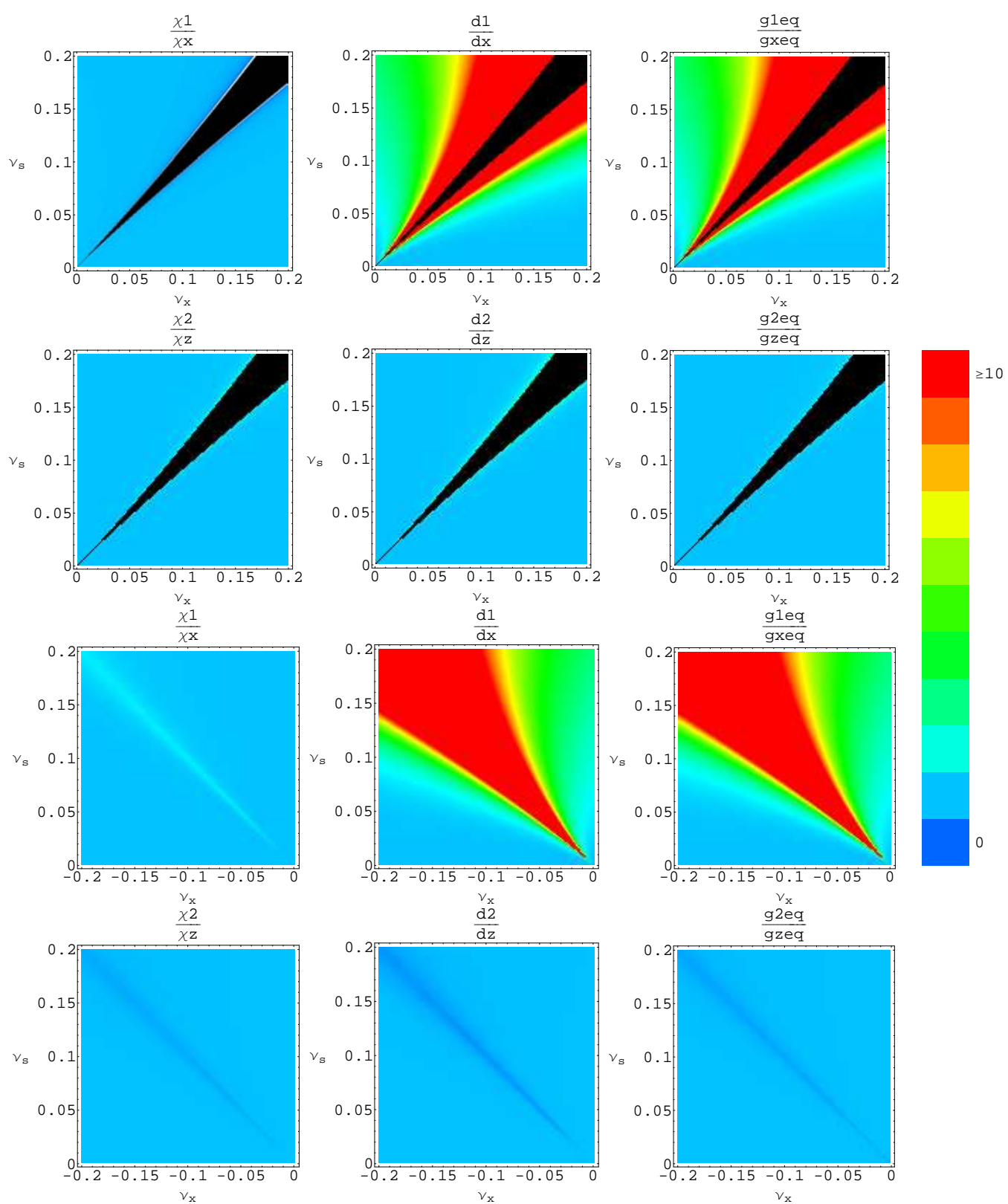

Figure 5.2: In this figure, we plot global damping, diffusion and equilibrium invariant values for coupling due to a dispersion of 1 meter at an RF cavity near the sum/difference resonances. The upper two rows are the sum resonance, and lower two are the difference resonance. Parameters are otherwise drawn from Table 5.3 based on the PEP-II LER. The quantities are plotted as a function of the betatron tune $\nu_{x}$ and the synchrotron tune $\nu_{s}$ which is positive and equal to $-\nu_{z}$, thus giving an inversion of sum and difference resonances. $\chi_{1}$ and $\chi_{2}$ are global damping decrements expressed in Table 5.2. $\bar{d}_{1}$ and $\bar{d}_{2}$ are global diffusion coefficients expressed in Table 5.1, and $\left\langle g_{1}\right\rangle_{\mathrm{eq}}$ and $\left\langle g_{2}\right\rangle_{\mathrm{eq}}$ are one half the ratio of these quantities as given by Eq.(4.10). All quantities have been divided by their uncoupled values so that the blue region with the value of 1 represents no effect from coupling. The region of instability due to the Hamiltonian dynamics is black. There is also an extremely small region of anti-damping instability outside the symplectic instability region for the sum resonance where the damping decrement $\chi_{1}$ is negative. This region is indicated by white. 

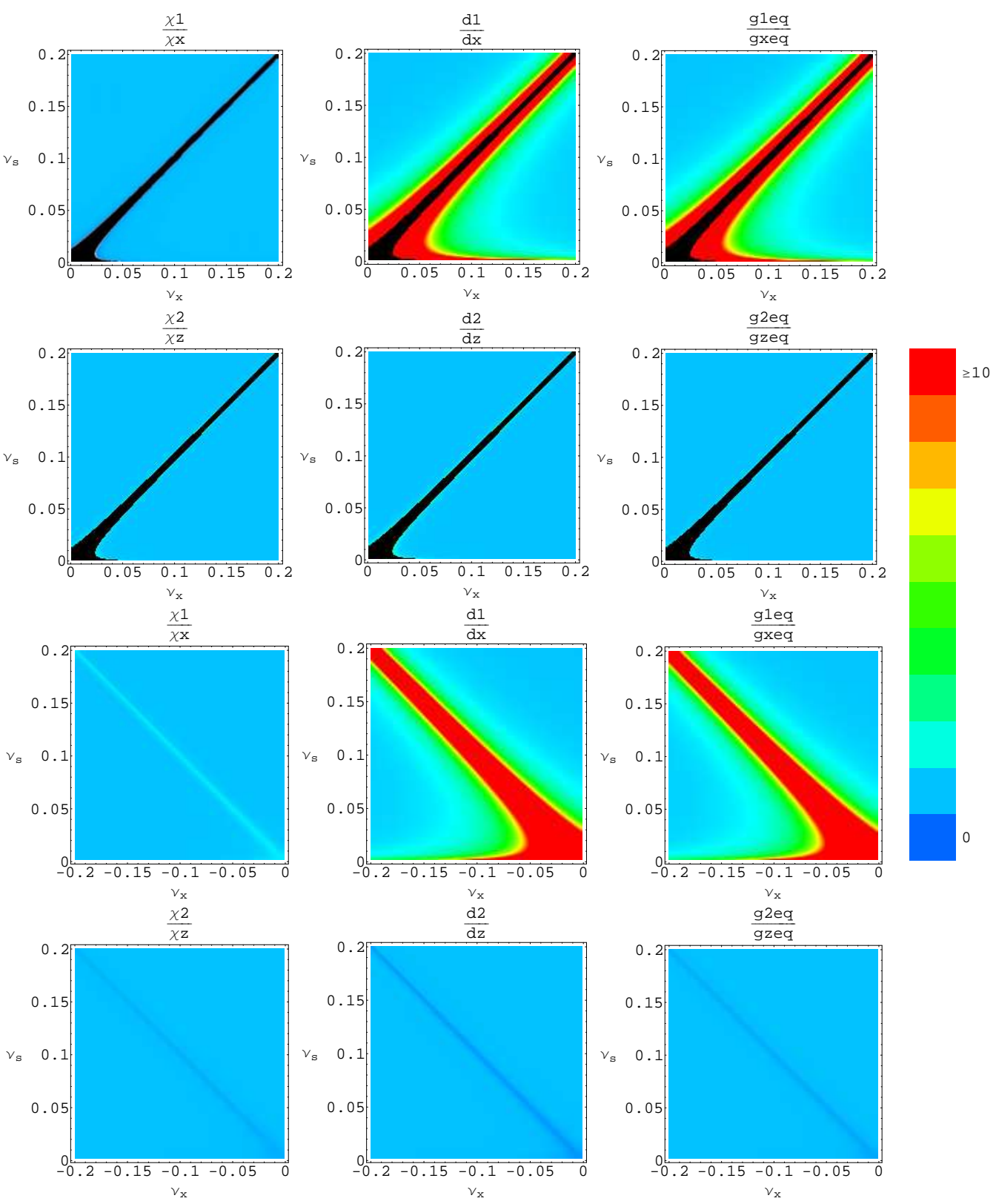

Figure 5.3: In this figure, we plot global damping, diffusion and equilibrium invariant values for coupling due to a crab cavity with $\xi_{c}=0.003$ near the sum and difference resonances. The dispersion at the crab cavity is set to 0 in this example. Because the coupling strength $\xi$ is inversely proportional to $\sqrt{\nu_{s}}$, the instability broadens for smaller $\nu_{s} . \chi_{1}, \chi_{2}, \bar{d}_{1}, \bar{d}_{2},\left\langle g_{1}\right\rangle$ and $\left\langle g_{2}\right\rangle$ are the same as in Fig.5.2. As in Fig. 5.2, the small region of anti-damping for $\chi_{1}$ near the sum resonance is colored white. 

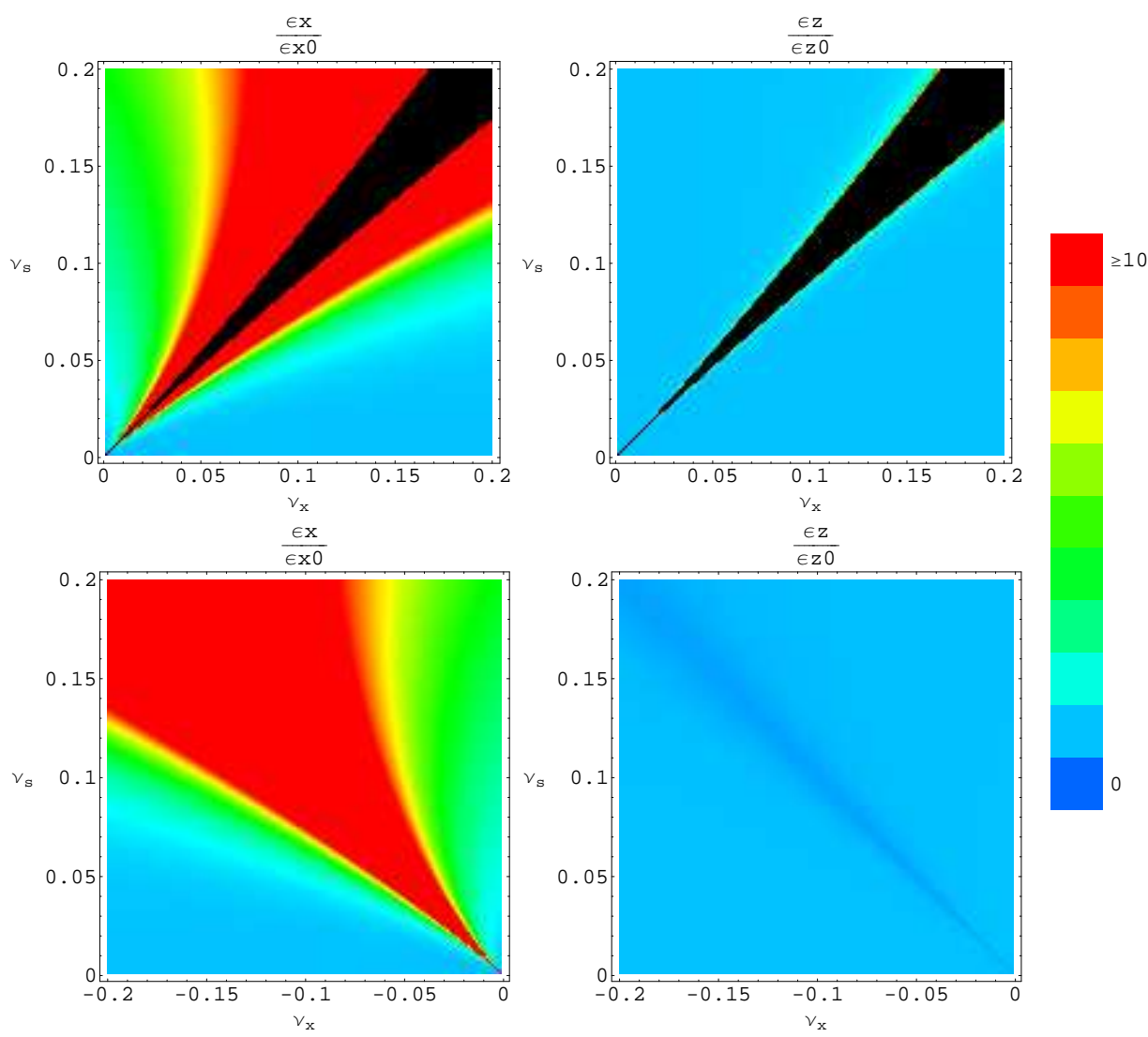

Figure 5.4: Projected emittances near the sum and difference resonances due to dispersion at the RF cavity. Paramaters are the same as those in Figs.5.2. The upper two plots are for the sum resonance with $\epsilon_{x, \text { pr }}^{+}$and $\epsilon_{z, \text { pr }}^{+}$defined in Eqs.(5.28) and (5.29). The lower two plots are for the difference resonance with $\epsilon_{x, \mathrm{pr}}^{-}$and $\epsilon_{z \text {,pr }}^{-}$ defined in Eqs.(5.31) and (5.32). 

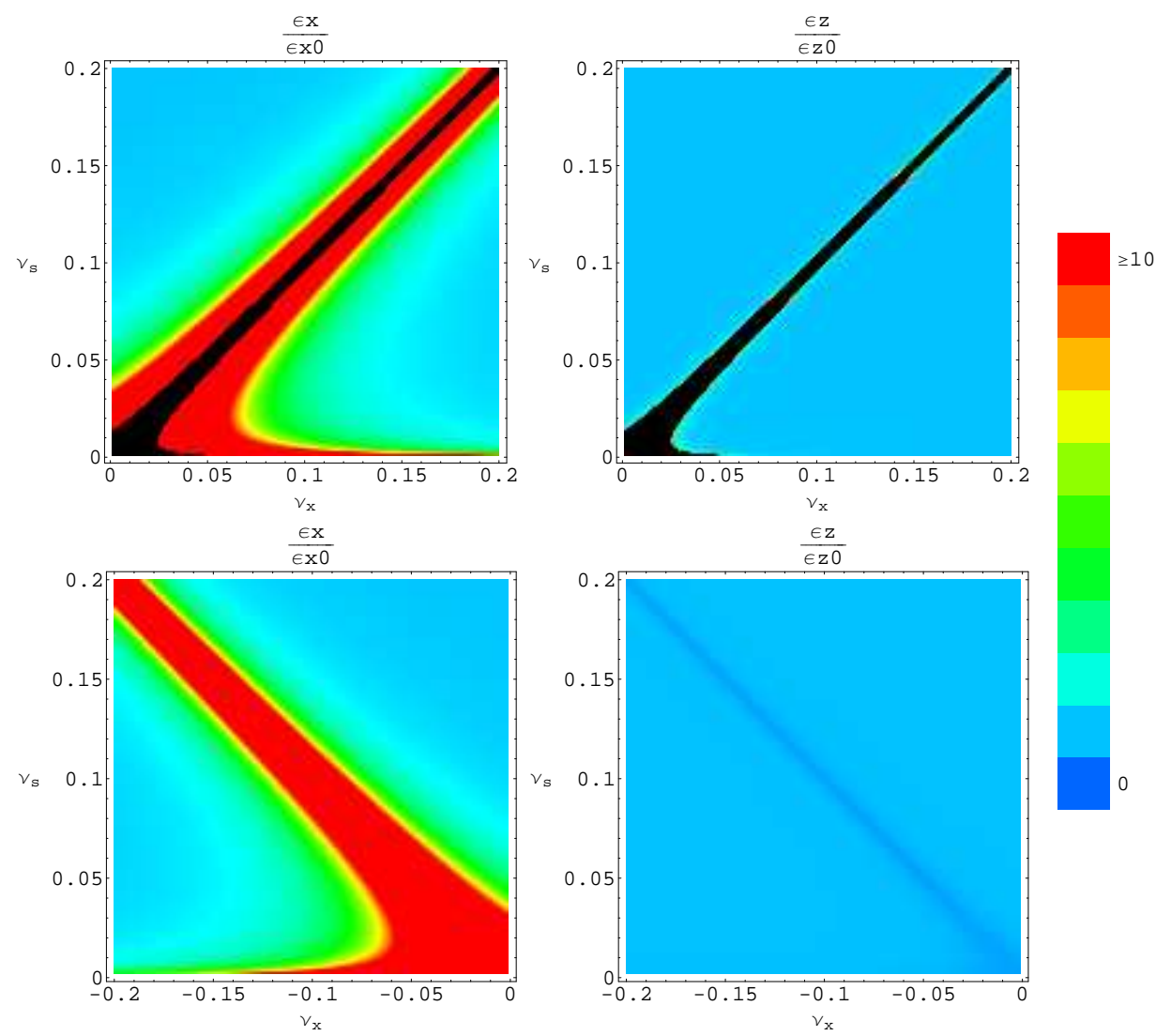

Figure 5.5: Projected emittances near the sum and difference resonances due to a crab cavity. The four plots have the same meanings as in Fig.5.4. Parameters are the same as those in Figs.5.3. 

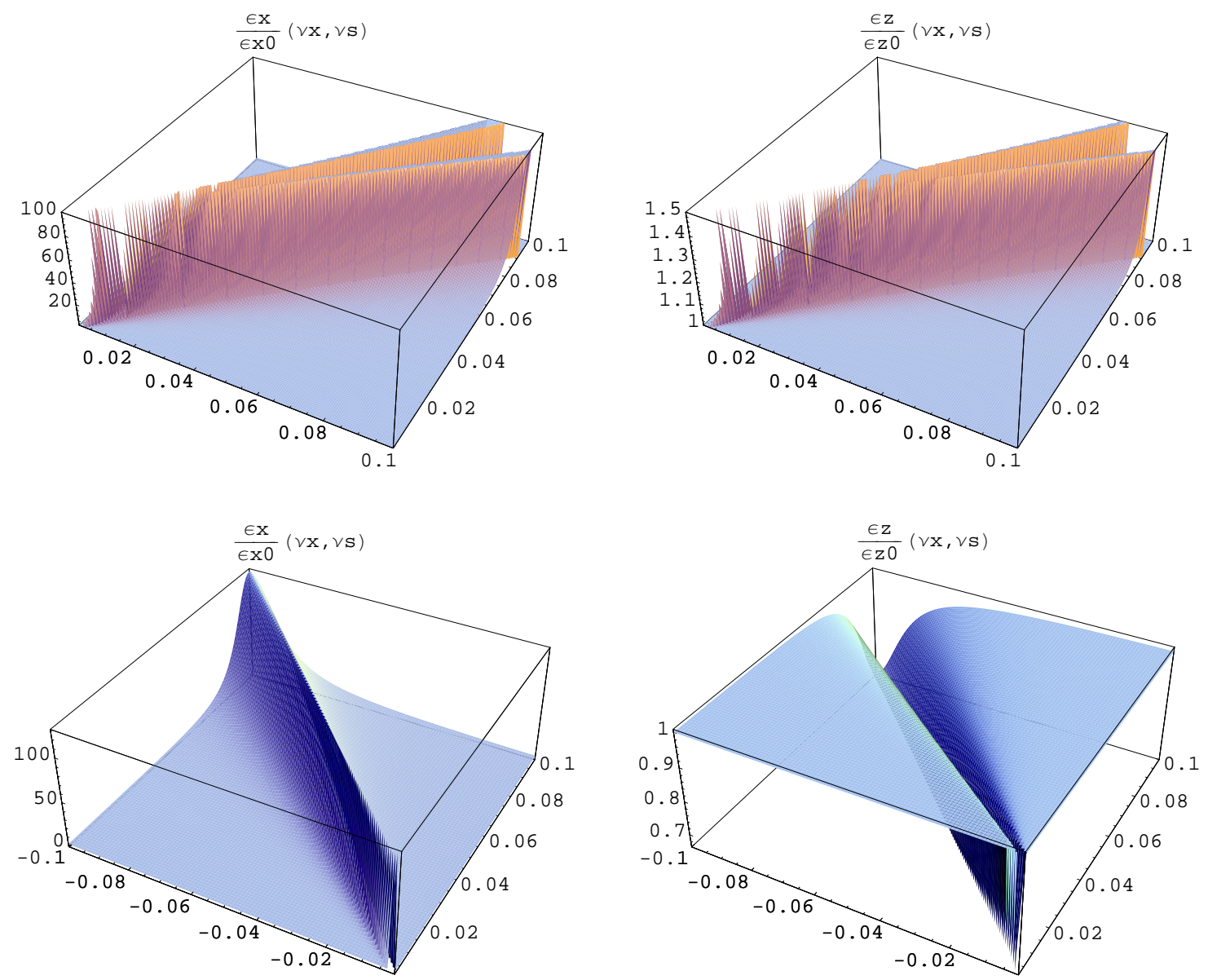

Figure 5.6: 3-D plots of projected emittances near the sum and difference resonances due to a dispersive RF cavity. The four plots have the same meanings as in Fig.5.4. Parameters are the same as those in Figs.5.2. 

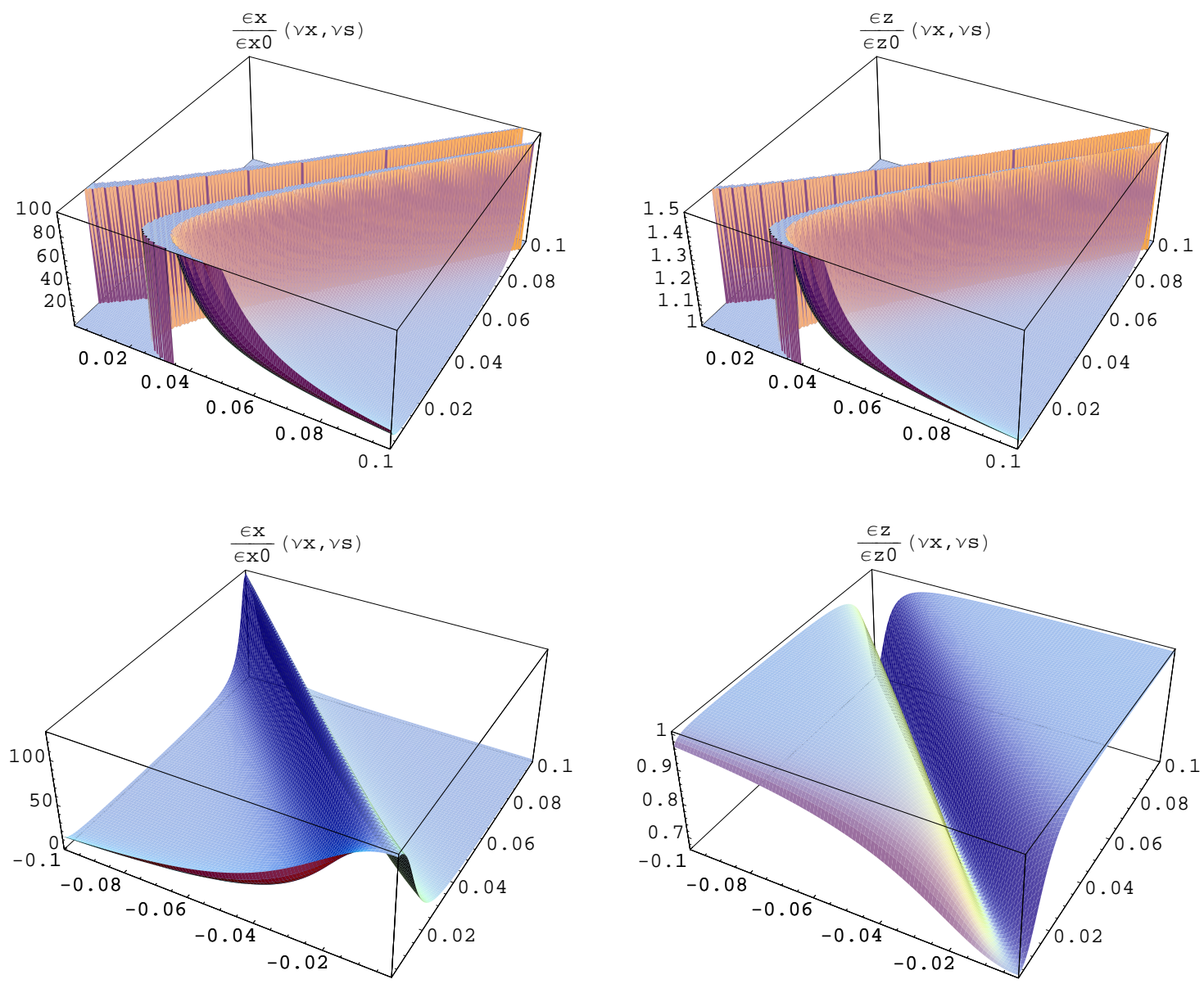

Figure 5.7: 3-D plots of projected emittances near the sum and difference resonances due to a crab cavity. The four plots have the same meanings as in Fig.5.4. Parameters are the same as those in Figs.5.3. 
examples. A natural way to affect this anti-damping region is to control the ratio $\chi_{z} / \chi_{x}$ through the damping partition number $\mathcal{D}$ as we see from Eqs.(4.50) and (4.51),

$$
\frac{\chi_{z}}{\chi_{x}}=\frac{\mathcal{J}_{z}}{\mathcal{J}_{x}}=\frac{2+\mathcal{D}}{1-\mathcal{D}}
$$

One can see from the values of $\chi_{x, z}$ in Table 5.3, that $\mathcal{D}$ is quite small with $\mathcal{D}=0.0056$. To explore the effect of changing $\mathcal{D}$ on the anti-damping instability, we multiply $\chi_{x}$ by $1-\mathcal{D}$ and $\chi_{z}$ by $(2+\mathcal{D}) / 2$ and plot $\chi_{1}$ for RF cavity dispersion and a crab cavity for various values of $\mathcal{D}$. The results are given in Fig.5.8 for $\mathcal{D}=0.25,0.5$, and 0.75 . The Hamiltonian dynamics instability is colored black, whereas the anti-damping instability is colored white.

\subsection{Dispersion at Crab Cavity}

Dispersion at the crab cavity affects all of the resonances. However, for the crab cavity near the sum and difference resonances, in our plots, we have set the dispersion to zero. For the case of $\nu_{x}$ near a half-integer, a case of particular interest, we have explored the effect of dispersion. In Fig.5.9, we plot stability diagrams (stable if $|\lambda|=1$, unstable otherwise) for the cases of no dispersion and a dispersion of 3 meter. We see that when the dispersion is turned on, the integer and half-integer $\nu_{x}$ resonances become visible, as well as a shifting of the integer $\nu_{z}$ resonance. We have also plotted the equilibrium horizontal emittance near the $\nu_{x}$ half-integer resonance as well as the equilibrium longitudinal emittance near the $\nu_{z}$ integer resonance. For the $\nu_{x}$ half-integer resonance, since the width is largely independent of $\nu_{s}$, but depends on the dispersion, we have plotted the horizontal emittance as a function of the tune $\nu_{x}$ and the dispersion $\eta$, showing a broadening effect for larger dispersion.

\subsection{Numerical Results}

Finally, we include an example of a numerical comparison between the eigenvalues obtained using our perturbation theory against those obtained numerically directly 

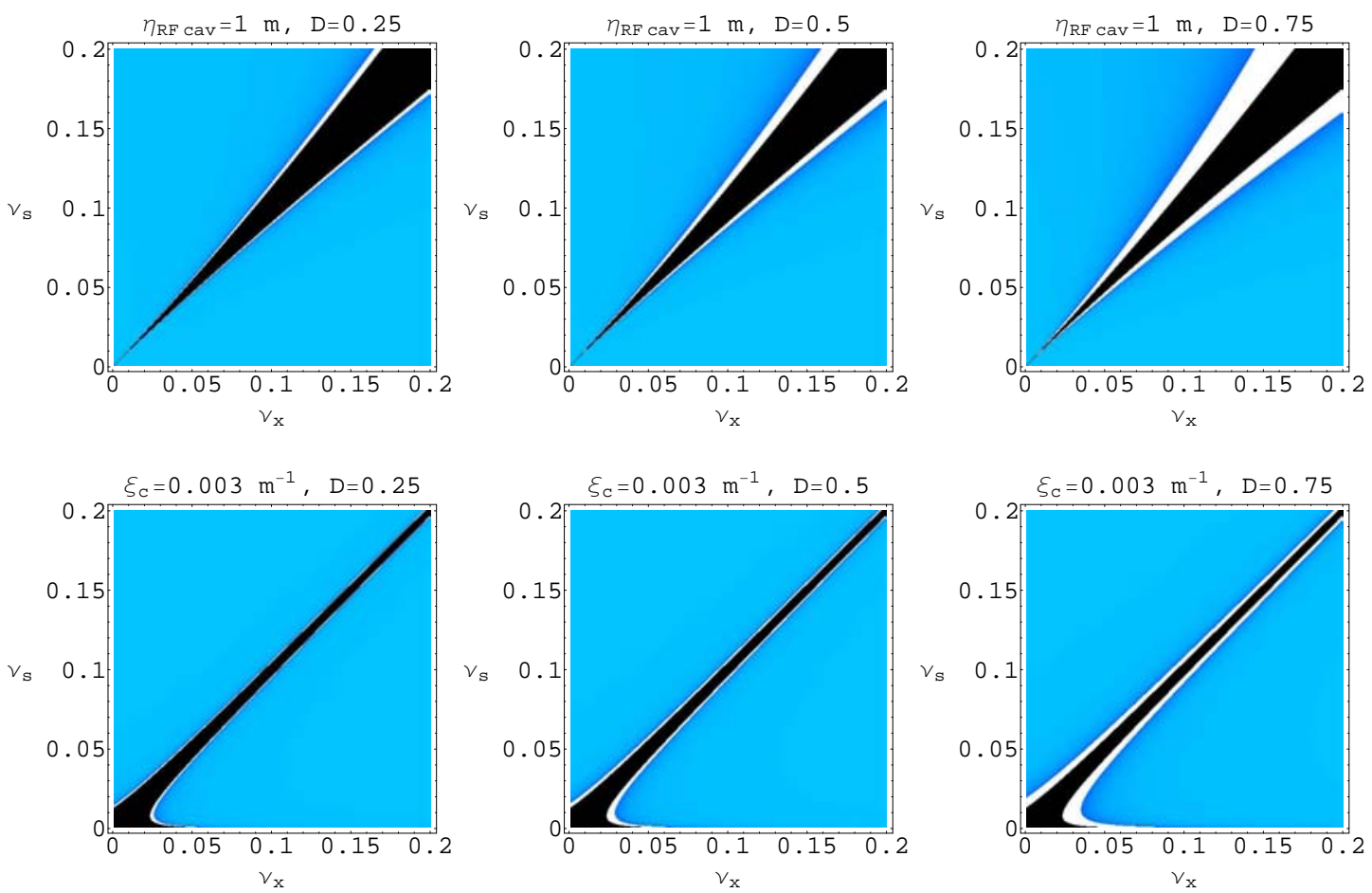

Figure 5.8: Damping decrement near sum resonance for coupling due to a dispersive RF cavity (upper plots) and a crab cavity (lower plots). The black region represents an instability in the Hamiltonian dynamics, whereas the white area represents a negative value of $\chi_{1}$ and hence an anti-damping instability. We vary the damping decrement $\mathcal{D}$. In the two left-most plots it is 0.25 . For the center plots it is 0.5 , and the right plots have 0.75. The plots of $\chi_{1}$ near the sum resonance in Figs.5.2 and 5.3 plot the same quantities, except that in those cases, $\mathcal{D} \approx 0$ as discussed in the text. Increasing $\mathcal{D}$ increases the ratio of $\chi_{z}$ and $\chi_{x}$ which widens the region where an anti-damping instability occurs. 

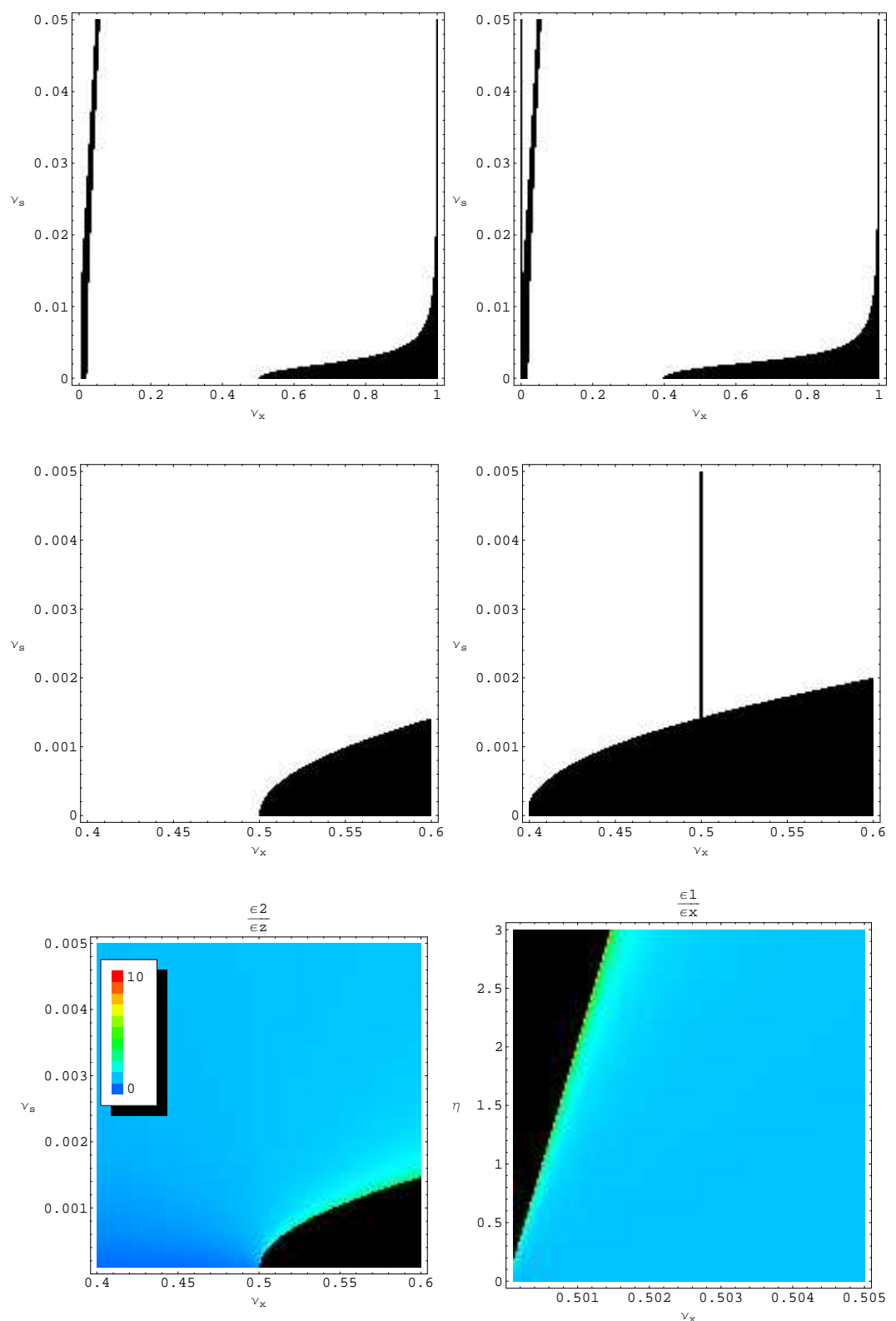

Figure 5.9: These six plots relate to crab cavity coupling. The upper two plots are instability diagrams. The eigenvalues $\lambda_{j}$, have been computed numerically for each value of the tunes and if for each $j,\left|\lambda_{j}\right|=1$, it is stable and colored white, whereas $\lambda_{j} \neq 1$ is unstable and colored black. The left plot has no dispersion at the crab cavity, while the right plot has a dispersion of 3 meters. The middle two plots show a magnified view of the upper two plots near $\nu_{x}=1 / 2$. Note that the $\nu_{x}$ half integer resonance caused by dispersion is only visible upon magnification. The integer $\nu_{x}$ resonance also appears with dispersion as can be seen from the thin black line near $\nu_{x}=0$ in the upper right plot. The bottom two plots give the horizontal and longitudinal equilibrium invariants, $\left\langle g_{1}\right\rangle_{\text {eq }}$ and $\left\langle g_{2}\right\rangle_{\text {eq }}$ divided by their uncoupled values, defined in Eq.(4.10). Because $\chi_{1,2}=\chi_{x, z}$ for integer and half-integer resonances, this is also given by the ratios $\bar{d}_{1} / \bar{d}_{x}$ and $\bar{d}_{2} / \bar{d}_{z}$ where $\bar{d}_{1}$ and $\bar{d}_{2}$ are defined in Table 5.1. Explicit expressions for the emittance growth for transverse and longitudinal are given in Eqs. (5.49) and (5.52). We have set the dispersion at the crab cavity to zero here. The bottom left is plotted as a function of $\nu_{x}$ and $\nu_{s}$, while the bottom right is plotted as a function of $\nu_{x}$ and dispersion at the crab cavity in units of meters. The $\nu_{x}$ half-integer instability does not depend on $\nu_{s}$. 
from the one-turn matrix. In Fig.5.10 we plot the computed versus numerical values of $\mu_{2}$ near the $\nu_{z}$ integer resonance, as a function of $\nu_{x}$ including a dispersion function of 0.1 meter at the crab cavity. The agreement is quite reasonable.

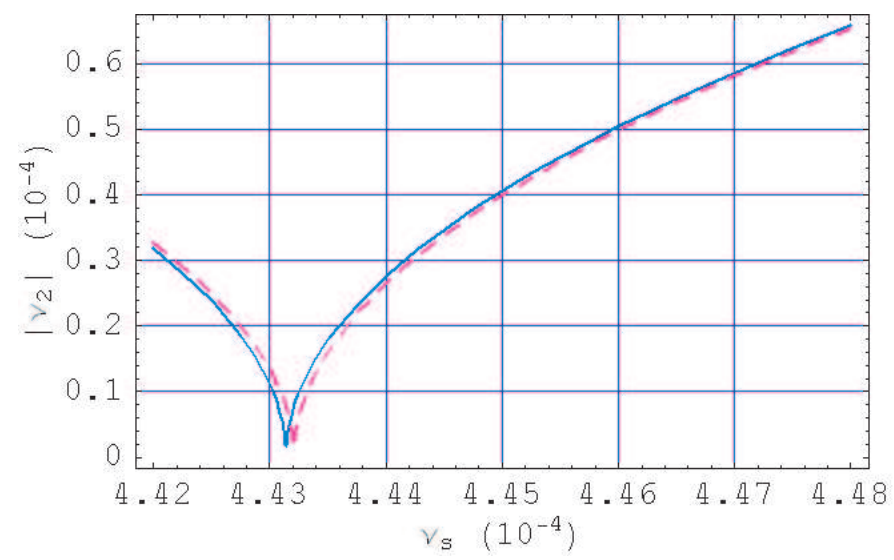

Figure 5.10: Comparison of eigenvalue formula to direct numerical calculation. Here, $\nu_{x}=0.51$, and we plot the absolute value of $\mu_{2}$, which becomes imaginary (unstable) due to the integer $\mu_{s}=0$ resonance. We have set the dispersion at the crab cavity to be $\eta=0.1 \mathrm{~m}$. The red is the numerical result, while the blue is Eq.(3.160).

\subsection{Instabilities Near Half-Integer $\nu_{x}$}

We are particularly interested in the case of the betatron tune just above the half integer because this is a case that is commonly used in colliders such as PEP-II and KEK-B. This means that we should pay attention to the $\nu_{x}$ half-integer resonance. We see that there is indeed such an instability for a dispersive RF cavity and for a crab cavity. For the dispersive RF cavity if we set $\alpha_{x}=\eta^{\prime}=0$ in Eq.(3.130), we find an instability for

$$
\frac{1}{2}<\nu_{x}<\frac{1}{2}+\frac{1}{2 \pi} \frac{\eta^{2}}{C \alpha_{c} \beta_{x}} \mu_{s}^{2}
$$

For the crab cavity, we also find a $\nu_{x}$ half-integer resonance. The condition is given by Eq.(3.156). When the dispersion at the crab cavity is negligible, the condition is

$$
\frac{1}{2}<\nu_{x}<\frac{a \beta_{x} \xi_{c}^{2}}{8 \pi} . \quad(\text { no dispersion })
$$


This is typically quite a narrow instability. When dispersion dominates, the condition becomes

$$
\left|\nu_{x}-\frac{1}{2}\right|<\frac{\eta \xi_{c}}{2 \pi} . \quad \text { (dispersion dominates) }
$$

In this case, near $\nu_{x}=1 / 2$, applying Eq. (5.25), and using $\Delta \mu \approx 2\left(\mu_{x}-\pi\right)$, the emittance will be increased by a factor of

$$
\frac{\epsilon_{1}}{\epsilon_{x}}=\frac{1}{\sqrt{1-\left(\frac{\eta \xi_{c}}{2 \pi\left(\nu_{x}-1 / 2\right)}\right)^{2}}}
$$

As an example, for the parameters in Table 5.3 with a dispersion of 1 meter at the crab cavity, when $\nu_{x}-1 / 2$ is 0.0016 , there is an increase in emittance of $10 \%$ due to this resonance.

In addition to the $\nu_{x}$ half integer resonance, because the synchrotron tune is typically small, one is also concerned with the $\nu_{z}$ integer resonance. In fact, this resonance is explored in [35]. Looking at Eq.(D.25), because of the factor $\cot \frac{\mu_{x}}{2}$ we expect an instability for this resonance, although very near $\nu_{x}=\frac{1}{2}$, it is quite narrow. Adding dispersion changes this simple picture. As we see in Fig.5.8, the dispersion moves the $\nu_{z}=0$ resonance into the region $\nu_{x}<\frac{1}{2}$, and for a fixed value of $\nu_{x}$ increases its width in $\nu_{s}$. The perturbed synchrotron tune due to the integer $\nu_{z}$ resonance is given by

$$
\nu_{2}=\sqrt{\nu_{s}^{2}+\left(\frac{C \alpha_{c} \beta_{x}}{4} \cot \left(\pi \nu_{x}\right)-\eta^{2}\right)\left(\frac{\xi_{c}}{2 \pi}\right)^{2}}
$$

from which we see that for $\nu_{x}$ just above $1 / 2$, there can be an instability when

$$
\nu_{s}<\frac{\xi_{c}}{2 \pi} \sqrt{\frac{C \alpha_{c} \beta_{x}}{4}\left(\nu_{x}-\frac{1}{2}\right)+\eta^{2}}
$$

which was also derived in [35]. For $\nu_{x}<\frac{1}{2}$, we see that this resonance can substantially increase the synchrotron tune.

Associated with this integer $\nu_{z}$ resonance is an increase or decrease of longitudinal emittance. Applying equation (5.26), and considering the case where the dispersion at the crab cavity is zero, we find that the longitudinal emittance is multiplied by a 
factor of

$$
\frac{\epsilon_{2}}{\epsilon_{z}}=\frac{\nu_{s}+\left[1+\operatorname{sgn}\left(\nu_{x}-\frac{1}{2}\right)\right] \frac{C \alpha_{c} \beta_{x}}{4 \nu_{s}} \cot \left(\pi \nu_{x}\right)\left(\frac{\xi_{c}}{2 \pi}\right)^{2}}{\sqrt{\nu_{s}^{2}+\frac{C \alpha_{c} \beta_{x}}{4} \cot \left(\pi \nu_{x}\right)\left(\frac{\xi_{c}}{2 \pi}\right)^{2}}} .
$$

We have used Eq. (3.51) and the fact that $\Delta \mu \approx 2 \mu_{z}$ for the integer $z$ resonance. The sgn (step) function comes from the $\cos \phi$ term in (3.51), where $\phi$ involves $\cot \left(\mu_{x} / 2\right)$ as we find from Table III. Note that the sgn function does not make this expression discontinuous at $\nu_{x}=1 / 2$, since $\cot (\pi / 2)=0$ and so there is no perturbation there.

One may also be interested to know the effects of dispersion at an RF cavity when the $\nu_{x}$ is near a half integer. For this case, we find a very weak $\nu_{x}$ half-integer stop band. This instability occurs when

$$
\frac{2 \pi \mathcal{H}_{x}}{C \alpha_{c}} \nu_{s}^{2}>\nu_{x}-\frac{1}{2}
$$

which is a small stop band both because of the $\nu_{s}^{2}$ and the $\mathcal{H}_{x}$ which is $\eta_{c}^{2} / \beta_{x}$ when $\eta^{\prime}=\alpha_{x}=0$, where $\eta_{c}$ is the dispersion at the RF cavity. Regarding the $\nu_{z}$ integer resonance, we find that it is absent for coupling due to a dispersive RF cavity. One can understand this fact by observing that the strength of the perturbation due to dispersion is proportional to $\nu_{s}^{2}$, so approaching $\nu_{s} \rightarrow 0$ effectively turns off the perturbation and avoids an instability.

Even when there is not an instability, we have seen that the emittances can be affected near a resonance. For the case of the $\nu_{x}$ half-integer resonance, the horizontal emittance can become large, whereas for the $\nu_{z}$ integer resonance, the longitudinal emittance (and correspondingly bunch length and energy spread) can become large. For the case of small synchrotron tune and betatron tune just above the half-integer a combination of these two effects is expected, i.e. a combination of the bottom two plots in Fig. 5.9.

\subsection{Instabilities Near Integer $\nu_{x}$}

In case of a storage ring operated near an integer $\nu_{x}$, one needs to be concerned with four resonances: integer $\nu_{x}$ and $\nu_{z}$, and the sum or difference resonances. Refer to 
the upper two plots in Fig.5.9 for a numerical example of the instability region. From our analytical expressions for the perturbed tunes, we learn that for small $\nu_{s}$, a crab cavity is particularly dangerous in this region because the effective coupling strengths for the sum and difference resonances $\xi_{ \pm}$scales as $1 / \sqrt{\nu_{s}}$. Regarding the integer $\nu_{z}$ resonance, $\nu_{x}$ above an integer is safer than below an integer because the $\nu_{z}$ integer resonance has a particularly large stop band just below integer $\nu_{x}$ coming from the factor of $\cot \left(\pi \nu_{x}\right)$ in Eq.(3.78). For $\nu_{x}$ above an integer, this factor causes increased stability, even reducing equilibrium longitudinal emittance, as seen in the dark blue lower left region of the bottom left plot of Fig.5.9. However, because of the strong $\nu_{x}=-\nu_{z}$ sum resonance, this effect would be washed out, at least for $\nu_{x}$ near the integer. Nevertheless, this result suggests the possibility of using coupling to increase stability and reduce emittance through careful tuning near a coupling-induced integer or half-integer resonance, perhaps in another context such as transverse coupling.

\subsection{Single Versus Multiple Resonances}

In this thesis, we have analyzed each resonance in isolation. This should be accurate as long as the tunes are such that they are much closer to one resonance than any other. Particularly near $\nu_{x}=\nu_{s}=0$ this can break down. In this situation, the system can be near three resonances, simultaneously: the integer $\nu_{x}$, the integer $\nu_{z}$, and either the sum or difference resonance. In this case, we expect that there may be greater errors in our expressions. In the case that one of the resonances does not cause an instability, but still can strongly affect the tune, such as for the difference resonance or the coupling $\nu_{z}$ integer resonance for $\nu_{x}<\frac{1}{2}$, one could use the perturbed, but not unstable tune as an input to determine the instability of the other tune. When the tunes are equally near to multiple resonances, a more elaborate analysis is necessary. We note that our perturbation theory can accommodate this situation (e.g. the quadruple degeneracy near $\nu_{x}=\nu_{z}=0$ ) but we do not pursue it further here. 


\subsection{Discussion}

In the presence of damping and diffusion mechanisms, such as synchrotron radiation, even near to such resonances, we have argued that the beam distribution can usually be described as a Gaussian function of the invariants of the one-turn map. Regarding the effect of coupling on this near resonance distribution, one needs to consider both the issues of stability and the effect on the equilibrium emittances.

Instability can occur in one of two ways. First, the coupling can cause one or two pairs of the eigenvalues to become complex, which for a symplectic matrix means that there will be a growing mode. Secondly, near a sum resonance, the coupling can mix together the damping rates in such a way that one of the modes has negative damping, which also indicates an instability.

Even if instability can be avoided, coupling near a resonance can have a substantial effect on the equilibrium emittances. This is particularly so if there is a large discrepancy between the emittances, which is indeed the typical case for synchrobetatron dynamics in which the horizontal emittance is much smaller than the longitudinal. For the case of PEP-II, $\epsilon_{z} / \epsilon_{x}=190$. Intuitively, one might expect, therefore, that synchrobetatron coupling would have a major effect on transverse emittance, since it is coupled to such a huge reservoir of longitudinal emittance. This is indeed what we find for both the sum and difference resonances. Near a sum resonance, although both emittances blow up at the resonance, a little bit off resonance one finds a large growth in the horizontal emittance $\epsilon_{x}$ and a very small growth in the longitudinal emittance $\epsilon_{z}$. Near a difference resonance, we also find major growth in $\epsilon_{x}$ whereas in this case there is a small decrease in $\epsilon_{z}$. The relationships between the emittances and how a change in one affects a change in the other can be understood by looking at the various sum rules we have derived in section 4.1. We have also derived (Eqs. (5.43) and (5.44)) sum rules for how the projected emittances relate to each other. We find qualitatively similar behavior for both the projected emittances and the emittances of the eigen-modes.

One might generalize the intuitive concept of coupling as a sort of equilibration between reservoirs and roughly state that when there is a large discrepancy between 
two quantities, coupling can have a strong effect near a resonance. We have just mentioned this effect for the case of the equilibrium emittances near sum/difference resonances. The other example where this is evident is in the previously mentioned anti-damping instability near a sum resonance. We have found that the larger the ratio of the damping rates, the larger the region of tune space that becomes unstable for a given coupling strength. A natural way in which this ratio is altered is through the damping partition number $\mathcal{D}$. We have seen that increasing $\mathcal{D}$ expands this region of the anti-damping instability.

It turns out, however that the intuition of the previous paragraph does not apply near integer and half-integer resonances. Although coupling can cause an instability when one of the tunes is near an integer or half integer, the damping rates are not affected much by this instability and although the diffusion coefficient for the unstable mode does get large and diverge at the instability, it does not mix with the other (stable mode) diffusion coefficient. Thus, although emittance is still affected by being near an integer or half-integer resonance, it is not as strong of an effect as with the sum and difference resonances where disparity between transverse and longitudinal damping and diffusion rates and emittances enhances the impact. 


\section{Chapter 6}

\section{Non-Constant Damping/Diffusion}

In Chapter 4, we considered the case where the diffusion and damping coefficients were independent of the phase space position. In that case, the emittance evolution equation reduced to Eq. (4.6) involving the global damping and diffusion for the invariants $\chi_{a}$ and $\bar{d}_{a}$. For the general case, we find that it is not particularly convenient to compute $\bar{d}_{a}$ and $\chi_{a}$. Instead we will compute the second moment changes $d \Sigma / d t$ directly using (2.73)

$$
\frac{d \Sigma_{i j}}{d t}=\int\left(\tilde{b}_{i} z_{j}+\tilde{b}_{j} z_{i}+\tilde{d}_{i j}\right) f(\vec{z}) d \vec{z}
$$

where $\tilde{b}_{i}$ and $\tilde{d}_{i j}$ are the damping and diffusion coefficients. Then, given the invariant matrices $G_{a}$, the total change in the average value of the invariant around the ring is given by

$$
\Delta\left\langle g_{a}\right\rangle=\frac{1}{\beta_{s} c} \int d s \operatorname{Tr}\left[G_{a} \frac{d \Sigma}{d t}\right]
$$

where we are integrating along the closed orbit and have used the relation $d s=\beta_{s} c d t$ with $\beta_{s} c$ the velocity of the particle along the orbit.

Now, in general, non-uniform damping and diffusion will give rise to a nonGaussian distribution. For the purposes of this thesis, however, we will assume that the distribution remains Gaussian. In particular, we recall that the distribution is a function of the invariants

$$
f(\vec{z})=\frac{1}{\pi^{3}\left\langle g_{1}\right\rangle\left\langle g_{2}\right\rangle\left\langle g_{3}\right\rangle} \exp \left(-\frac{g_{1}}{\left\langle g_{1}\right\rangle}-\frac{g_{2}}{\left\langle g_{2}\right\rangle}-\frac{g_{3}}{\left\langle g_{3}\right\rangle}\right)
$$


$G_{a}$ thus enters in two places: in (6.2) and in Eq. (6.3) for $f(z)$ which is required in the computation of $\tilde{b}_{a}$ and $\tilde{d}_{a b}$.

Actually, we will find that the $G_{a}$ can enter in a third place in this calculation: in the computation of the damping and diffusion $\tilde{b}_{i}$ and $\tilde{d}_{i j}$. This will be the case for intrabeam scattering, our main example for this chapter.

\subsection{IBS Dissipation/Diffusion}

IBS is a well studied phenomenon in which scattering of beam particles off of one another causes a growth in the emittance. We revisit this topic, providing a firstprinciples derivation based on the framework we have developed in this thesis. Our formulation has two benefits. The first is that it has no large distance divergence which usually leads to a Coulomb Logarithm. In this chapter, we derive our expressions and discuss the connection to existing approaches. Within the general framework of this thesis, our formulation also has the benefit of being extendable to the coupled case. We simply use the coupled invariants $G_{a}$. We discuss this generalization in the following chapter.

Let us recall the equations of Chapter 2:

$$
\begin{aligned}
\tilde{b}_{i} & =\dot{n} \int d \Phi g(\Phi) \delta z_{i}(\Phi) \\
\tilde{d}_{i j} & =\dot{n} \int d \Phi g(\Phi) \delta z_{i}(\Phi) \delta z_{j}(\Phi)
\end{aligned}
$$

Here, $\dot{n}$ is an average value for the number of kicks per unit time. $\delta z_{i}$ is the magnitude of a given kick, $\Phi$ is the parameter that the kick depends upon, and $g(\Phi)$ is the normalized distribution of $\Phi$. In the case of IBS, the kicks are caused by other particles in the beam.

In order to compute the kick $\delta z_{i}$, it is easier to work in the beam frame ${ }^{1}$. To do this, we must transform all of these equations. It turns out that this is not hard to do, as long as we assume that the motion is non-relativistic in the beam frame. In

\footnotetext{
${ }^{1}$ For further understanding of relativistic effects see e.g. [8, 55].
} 
particular, if $\vec{Z}$ is the phase space vector in the beam frame, it is related to the phase space vector in the lab frame by

$$
\vec{z}=\mathcal{L} \vec{Z}
$$

with

$$
\left(\begin{array}{c}
x \\
y \\
z \\
x^{\prime} \\
y^{\prime} \\
\delta
\end{array}\right)=\left(\begin{array}{cccccc}
1 & 0 & 0 & 0 & 0 & 0 \\
0 & 1 & 0 & 0 & 0 & 0 \\
0 & 0 & \frac{1}{\gamma} & 0 & 0 & 0 \\
0 & 0 & 0 & \frac{1}{P_{0}} & 0 & 0 \\
0 & 0 & 0 & 0 & \frac{1}{P_{0}} & 0 \\
0 & 0 & 0 & 0 & 0 & \frac{\gamma}{P_{0}}
\end{array}\right)\left(\begin{array}{c}
X \\
Y \\
Z \\
P_{X} \\
P_{Y} \\
P_{Z}
\end{array}\right)
$$

Here, $\gamma$ is the relativistic energy factor and $P_{0}$ is the longitudinal momentum of the beam. Note that we have reordered the phase space vector so that the position and momentum are lumped together. We denote the phase space vector in the beam frame by $\vec{Z}=(\vec{X}, \vec{P})$. We let $\vec{f}(\vec{X}, \vec{P})$ be the phase space distribution for the particle beam in the beam rest frame. We normalize both $\bar{f}$ and $f$ to 1 . That is

$$
\int \vec{f}(\vec{Z}) d \vec{Z}=\int f(\vec{z}) d \vec{z}=1
$$

Using the fact that $\operatorname{det}(\mathcal{L})=1 / P_{0}^{3}$, we derive that

$$
\bar{f}(\vec{Z})=\frac{f(\vec{z})}{P_{0}^{3}}
$$

This accounts for the transformation of $f$ to the beam frame. Now let us consider Eqs. (6.4) and (6.5) as being in the beam frame. That is, we replace $\delta z_{i}$ with $\delta Z_{i}$. $\Phi$ will then refer to $\vec{Z}_{2}$, the distribution of particle 2 that scatters off the given particle, and $g(\Phi)=\bar{f}(\vec{Z})$. To be explicit:

$$
\begin{aligned}
\tilde{b}_{i} & =\dot{n} \int d \Phi g(\Phi) \delta Z_{i}(\Phi) \\
\tilde{d}_{i j} & =\dot{n} \int d \Phi g(\Phi) \delta Z_{i}(\Phi) \delta Z_{j}(\Phi)
\end{aligned}
$$

Since we will have no need for diffusion and damping coefficients in the lab frame, 
in this chapter, $\tilde{b}_{i}$ and $\tilde{d}_{i j}$ will always be assumed to be for the beam frame. We also note that only when $i, j$ represent momentum indices will they be non-zero. Let $a, b=x, y, z$ represent momentum indices. Then, $\tilde{b}_{a}$ involves the change of $P_{a}$, etc. Now, we also need to account for the time dilation factor,

$$
d t=\gamma d \bar{t}
$$

where $d \bar{t}$ is the time increment measured in the beam frame. Now, to transform Eq. (6.1), we consider the moments in the beam frame:

$$
\bar{\Sigma}_{i j}=\left\langle Z_{i} Z_{j}\right\rangle=\int d \vec{Z} Z_{i} Z_{j} \bar{f}(\vec{Z})
$$

from which we can show that

$$
\Sigma=\mathcal{L} \bar{\Sigma} \mathcal{L}^{T}
$$

Thus, Eq. (6.1) becomes

$$
\frac{d \bar{\Sigma}_{i j}}{d \bar{t}}=\int\left(\tilde{b}_{i} Z_{j}+\tilde{b}_{j} Z_{i}+\tilde{d}_{i j}\right) \bar{f}(\vec{Z}) d \vec{Z}
$$

We reiterate that we are computing $\tilde{b}_{i}$ and $\tilde{d}_{i j}$ in the beam frame. They will depend on $\vec{Z}$ through their dependence on $\bar{f}$. Now, writing $\bar{G}_{a}$ for $G_{a}$ in the beam frame, we have

$$
\bar{G}_{a}=\mathcal{L}^{T} G_{a} \mathcal{L}
$$

So that finally,

$$
\Delta\left\langle g_{a}\right\rangle=\frac{1}{\gamma \beta_{s} c} \int d s \operatorname{Tr}\left[\bar{G}_{a} \frac{d \bar{\Sigma}}{d \bar{t}}\right]
$$

Where $d \bar{\Sigma} / d \bar{t}$ was given in Eq. (6.15).

What remains is to compute $\tilde{b}_{i}$ and $\tilde{d}_{i j}$ and combine them into $d \bar{\Sigma} / d \bar{t}$. Now that we have established the connection between the lab and beam frames, we will drop the bar on the time variable, the extra factor of $\gamma$ contained in Eq. (6.17).

We compute $\tilde{b}_{i}$ and $\tilde{d}_{i j}$ from (6.10) and (6.11). Here is the picture this formulation implies. We have a particle at position $\vec{Z}_{1}=\left(\vec{X}_{1}, \vec{P}_{1}\right)$ scattering off of the other 
particles in the beam at phase space position $\left(\vec{X}_{2}, \vec{P}_{2}\right)$. If we interpret $\Phi$ to be $\vec{Z}_{2}$ and $g(\Phi)$ to be related to $\vec{f}\left(\vec{Z}_{2}\right)$, then what is the meaning of $\delta \vec{Z}$ ? Well, as long as $\vec{X}_{1}$ and $\vec{X}_{2}$ are far enough away, then we can consider them to be undergoing a scatter starting at infinity with an appropriate impact parameter. What is that impact parameter? If we define $\vec{r}=\vec{X}_{1}-\vec{X}_{2}$ and $\vec{\Delta}=\vec{P}_{1}-\vec{P}_{2}$. Then if the two particles were to continue in straight line paths, we can show that the distance of minimum approach is given by

$$
\vec{b}=\vec{r}-r(\hat{\Delta} \cdot \hat{r}) \hat{\Delta}
$$

with $\vec{\Delta}=\Delta \hat{\Delta}$ and $\vec{r}=r \hat{r}$, with hats indicating unit vectors. We take this to be the impact parameter for the scatter. It has a magnitude of

$$
b=r \sin \chi
$$

with

$$
\hat{\Delta} \cdot \hat{r}=\cos \chi
$$

So we found the impact parameter. What good does this do us? Well, given the Coulomb interaction, we can relate the impact parameter to the scattering angle as

$$
\tan \left(\frac{\psi}{2}\right)=\frac{2(m c)^{2} r_{0}}{\Delta^{2} b}=\frac{2 k}{\Delta^{2} b} \quad k=(m c)^{2} r_{0}
$$

And in terms of this scattering angle $\psi$, the change in momentum is given by

$$
\delta \vec{P}_{1}=\frac{1}{2} \Delta((\cos \psi-1) \hat{\Delta}+\sin \psi \hat{b})
$$

Now, we have assumed that the stochastic process will be a small perturbation to the underlying Hamiltonian dynamics. This implies that we assume that small angle scattering dominates. In the case of small angles, we find

$$
\delta \vec{P}_{1} \approx-\frac{4 k^{2}}{\Delta^{3} b^{2}} \hat{\Delta}+\frac{2 k}{\Delta b} \hat{b} \quad \psi<<1
$$

This equation says that the particle will receive a kick in opposite direction to its 
relative momentum with another particle and in the same direction as its vector impact parameter with that particle. The first of these terms causes a damping effect tending to slow particles down in their direction of travel and is termed "dynamical friction". The second term causes both the space charge and diffusive effects.

So we have computed $\delta \vec{Z}_{1}$. Of course, in physical coordinates, only $\delta \vec{P}_{1}$ is nonzero. In terms of indices, we write this as $\delta \vec{P}_{a}$ where $a=x, y, z$. Likewise, we denote $\tilde{b}_{i}$ and $\tilde{d}_{i j}$ as $\tilde{b}_{a}$ and $\tilde{d}_{a b}$ where the $a$ and $b$ represent the momentum indices. We find $\delta P_{a}$ to be a function of $\vec{Z}_{2}$, although it is in fact only a function of $\vec{Z}_{1}-\vec{Z}_{2}$, a fact which will help us later. Identifying $\Phi$ in $g(\Phi)$ with $\vec{Z}_{2}$ then, what is the function $g$ ? It is supposed to be the distribution of $\vec{Z}_{2}$ causing kicks within a time $\Delta t$. Given our picture of the scatter occurring at the point of minimum approach, it makes sense to just consider those particles with a $\vec{Z}_{2}$ such that the scatter occurs within $\Delta t$. We can compute the time to the distance of minimum approach to be

$$
t_{\min }=-\frac{m r}{\Delta} \hat{\Delta} \cdot \hat{r}
$$

This allows us to partition the distribution and only include that region that undergoes scattering such that

$$
0<t_{\min }<\Delta t
$$

or

$$
0<\cos \chi<\frac{\Delta}{m r} \Delta t
$$

Thus, we take

$$
g\left(\vec{Z}_{2}\right)=\frac{1}{\Gamma_{\Delta t}} \bar{f}\left(\vec{Z}_{2}\right) \Theta_{\Delta t}
$$

where

$$
\Theta_{\Delta t}= \begin{cases}1 & \text { if } 0<t_{\text {min }}<\Delta t \\ 0 & \text { otherwise }\end{cases}
$$

The quantity $\Gamma_{\Delta t}$ is the phase space volume contained in Eq. (6.25). It is inserted such that $g\left(\vec{Z}_{2}\right)$ is normalized:

$$
\int d \vec{Z}_{2} g\left(\vec{Z}_{2}\right)=1
$$


Finally we need $\dot{n}$. Let us refer to the total phase space volume as $\Gamma_{\text {tot }}$. If there are a total number of $N$ particles, then $\dot{n}$ is given by

$$
\dot{n}=\frac{N \frac{\Gamma_{\Delta t}}{\Gamma_{\text {tot }}}}{\Delta t}
$$

In words, it is the number of scatters per time $\Delta t$. Putting this all together and noting the cancelation of the factor of $\Gamma_{\Delta t} / \Gamma_{\text {tot }}$ in $\dot{n}$ we find

$$
\begin{aligned}
\tilde{b}_{a} & =\frac{N}{\Delta t} \int_{\Gamma_{\Delta t}} d \vec{Z} \bar{f}(\vec{Z}) \delta P_{a}(\vec{Z}) \\
\tilde{d}_{a b} & =\frac{N}{\Delta t} \int_{\Gamma_{\Delta t}} d \vec{Z} \bar{f}(\vec{Z}) \delta P_{a}(\vec{Z}) \delta P_{b}(\vec{Z})
\end{aligned}
$$

Now, since we will want $\Delta t \rightarrow 0$, we can replace the constraint on the range of integration by a delta function using the equation (valid for continuous $f$ )

$$
\lim _{\epsilon \rightarrow 0}\left\{\int_{0}^{\epsilon} f(y) d y=\int_{-1}^{1} \epsilon \delta(y) f(y) d y\right\}
$$

Using $\epsilon=\frac{\Delta}{m r} \Delta t$ and $y=\cos \chi=\hat{\Delta} \cdot \hat{r}$, and shifting the integration from $\vec{Z}=(\vec{X}, \vec{P})$ to $\vec{\eta}=(\vec{r}, \vec{\Delta})$ we get $^{2}$

$$
\begin{aligned}
\tilde{b}_{a}\left(\vec{Z}_{1}\right) & =N \int_{\Gamma_{\text {tot }}} d \vec{\eta} \frac{\Delta}{m r} \bar{f}\left(\vec{Z}_{2}\right) \delta P_{a}(\vec{\eta}) \delta(\hat{\Delta} \cdot \hat{r}) \\
\tilde{d}_{a b}\left(\vec{Z}_{1}\right) & =N \int_{\Gamma_{\text {tot }}} d \vec{\eta} \frac{\Delta}{m r} \bar{f}\left(\vec{Z}_{2}\right) \delta P_{a}(\vec{\eta}) \delta P_{b}(\vec{\eta}) \delta(\hat{\Delta} \cdot \hat{r})
\end{aligned}
$$

This change of integration variables from $\vec{Z}_{2}$ to $\vec{\eta}$ is particularly convenient because

\footnotetext{
${ }^{2}$ Actually, we really have a delta function of the form $\delta\left(\frac{\Delta}{r} \hat{r} \cdot \hat{\Delta}\right)$. We can write this as a sum over delta functions with the argument going to zero in the different regions. We ignore the $r \rightarrow \infty$ zero because it will never be reached in the integration with a distribution that goes to zero outside a finite region. So we have

$$
\delta\left(\frac{\Delta}{r} \hat{r} \cdot \hat{\Delta}\right)=\frac{r}{\Delta} \delta(\hat{r} \cdot \hat{\Delta})+\frac{r}{\hat{r} \cdot \hat{\Delta}} \delta(\Delta)
$$

The second term has one more power of $\Delta$ than the first one does and this will cause this term to be zero at the $\Delta \rightarrow 0$ limit. So we can safely ignore this term and we are left with the result above.
} 
of the fact that $\delta P_{i}$ depends only on $\vec{\eta}$ as we have indicated in these expressions.

Now, substituting (6.23) into (6.35) and (6.36), we have

$$
\tilde{b}_{a}=N \int d \vec{\eta} \frac{\Delta}{m r}\left[-\frac{4 k^{2}}{\Delta^{3} b^{2}} \hat{\Delta}_{a}+\frac{2 k}{\Delta b} \hat{b}_{a}\right] \delta(\hat{\Delta} \cdot \hat{r})
$$

and

$$
\tilde{d}_{a b}=N \int d \vec{\eta} \frac{\Delta}{m r}\left[\frac{16 k^{4}}{\Delta^{6} b^{4}} \hat{\Delta}_{a} \hat{\Delta}_{b}-\frac{8 k^{3}}{\Delta^{4} b^{3}}\left(\hat{\Delta}_{a} \hat{b}_{b}+\hat{\Delta}_{b} \hat{b}_{a}\right)+\frac{4 k^{2}}{\Delta^{2} b^{2}} \hat{b}_{a} \hat{b}_{b}\right] \delta(\hat{\Delta} \cdot \hat{r})
$$

At this point we would like to apply the irreversibility criterion discussed in Chapter 2, namely Eqs. (2.75) and (2.76) which say that $\tilde{b}_{a}$ should be antisymmetric under $\vec{P} \rightarrow-\vec{P}$ and $\tilde{d}_{a}$ should be symmetric. This criterion removes the second term in $\tilde{b}_{a}$ and the middle term in $\tilde{d}_{a b}$. The second term in $\tilde{b}_{a}$ causes the space charge effect, which we ignore, as it is not the focus of this study. In the usual treatment of this problem, only terms proportional to $\frac{1}{b^{2}}$ are kept. After using the $\delta$ function, we have $b=r$ and so these terms have a $\frac{1}{r}$ behavior if we ignore the distribution. One might think that the $\frac{\hat{b}}{b}$ term in $\delta p_{a}$ would dominate. However, if we ignore the distribution, then the integral over $\alpha$ of $\hat{b}$ is 0 . In our case where we have an explicitly varying distribution, this integral will no longer be 0 . This term gives the space charge force which we will ignore here. These arguments give the same result except for the first term in $\tilde{d}_{a b}$. This term we expect to be a symptom from using the small angle approximation and will ignore it. We note that if one continues the small angle expansion, there would be many additional such terms. Thus, we will compute

$$
\tilde{b}_{a}=-\frac{4 N k^{2}}{m} \int d \vec{\eta} \frac{\hat{\Delta}_{a}}{\Delta^{2} b^{3}} \bar{f}\left(\vec{Z}_{2}\right) \delta(\hat{\Delta} \cdot \hat{r})
$$

and

$$
\tilde{d}_{a b}=\frac{4 N k^{2}}{m} \int d \vec{\eta} \frac{\hat{b}_{a} \hat{b}_{b}}{\Delta b^{3}} \bar{f}\left(\vec{Z}_{2}\right) \delta(\hat{\Delta} \cdot \hat{r})
$$

Equations (6.39) and (6.40) are our general expressions for the damping and diffusion coefficients. We can use them with (6.15) to find the evolution of the second moments. Before doing so, however, let us briefly consider these quantities on their 
own terms. If we were to consider a generalization of our framework in which we allow for non-Gaussian beams, then we would want to solve the Fokker-Planck equation. This takes the form

$$
\frac{\partial \bar{f}}{\partial t}=\frac{\partial}{\partial Z_{i}}\left(-\tilde{B}_{i} f+\frac{1}{2} \frac{\partial}{\partial Z_{j}} \tilde{d}_{i j} \bar{f}\right)
$$

where $\tilde{B}_{i}$ will contain a term from the Hamiltonian dynamics in addition to the $\tilde{b}_{a}$ we are computing here. We have assumed that repeated indices are summed over.

These damping and diffusion coefficients involve a 6-D phase space integration. Because we have made the small angle approximation, we find that the short distance spatial integration will diverge logarithmically at short distances. We impose a minimum impact parameter cut-off of

$$
b_{\min }=\frac{2 k}{\Delta^{2}}
$$

This is also (within a factor of 2) the distance of minimum approach for the two particles assuming they approach each other head-on. The existence of this divergence and cut-off may seem alarming, but actually it really is just an artifact of having made the small angle approximation. If one does not make the small angle approximation and instead uses the full expressions given by (E.10) and (E.11), there is no short distance (or large $\psi$ ) divergence, but the result is approximately the same as if one uses the cut-off of (6.42). The varying of the minimum impact parameter with $\Delta$ makes calculations more difficult, and since it only will occur logarithmically, we can replace the $\Delta^{2}$ with an average value. This approximation is made by BjorkenMtingwa[13], but is not made by Piwinski[12]. We will face a similar issue when we combine the damping and diffusion into second moment evolution equations. If we keep the $\Delta$ dependence in $b_{\min }$, and make the CLA when computing the moment evolution for a Gaussian distribution, we arrive at the results of Piwinski. We will drop the $\Delta$ dependence and as a result will arrive at the results of Bjorken-Mtingwa after applying the CLA. We take a typical value of $\Delta$ given by the transverse velocity. Relating this to quantities in the lab frame, if we use the horizontal RMS velocity, 
then

$$
b_{\min }=\frac{r_{0} \beta_{x}}{\gamma^{2} \epsilon_{x}}
$$

with $\beta_{x}$ and $\epsilon_{x}$ the horizontal beta function and emittance. Using the longitudinal velocity would give

$$
b_{\min }=\frac{r_{0} \beta_{z}}{\gamma \epsilon_{x}}
$$

The single factor of $\gamma$ instead of $\gamma^{2}$ reflects the longitudinal Lorentz boost which affects $P_{z}$ but not $P_{x, y}$. Thus, in the typical case, the RMS $P_{z}$ is much smaller than $P_{x, y}$ and thus the corresponding $b_{\min }$ using $P_{z}$ is much larger. However, the transverse phase space will dominate the longitudinal, being of larger dimensionality, and we will thus use the transverse $b_{\min }$ of (6.43). In the case where the horizontal and vertical divergences differ by orders of magnitude, this assumption should be reexamined.

Let us suppose that the phase space distribution $\vec{f}(\vec{Z})$ separates into a product of functions of position and momentum:

$$
\bar{f}(\vec{Z})=\rho_{x}(\vec{X}) \rho_{p}(\vec{P})
$$

In this case, we find

$$
\tilde{b}_{a}=-\frac{N k^{2}}{4 m} 2 \pi L_{c} \bar{f}\left(\vec{Z}_{1}\right) \int d \vec{\Delta} \frac{\hat{\Delta}_{a}}{\Delta^{2}} \rho_{p}\left(\vec{p}_{2}\right)
$$

and

$$
\tilde{d}_{a b} \approx \frac{N k^{2}}{4 m} 2 \pi L_{c} \bar{f}\left(\vec{Z}_{1}\right) \int d \vec{\Delta} \rho_{p}\left(\vec{p}_{2}\right) \frac{\left(\delta_{a b}-\hat{\Delta}_{a} \hat{\Delta}_{b}\right)}{\Delta}
$$

We have been required to cut off the logarithmically divergent spatial integral at maximum and minimum impact parameters and have introduced the Coulomb Logarithm

$$
L_{c}=\ln \frac{b_{\max }}{b_{\min }}
$$

These correspond to the Rosenbluth potentials [10], also known as Landau collision integrals[11]. We will refer to the procedure of replacing the spatial distribution with a uniform density and cutting off the resulting divergences incorporating them into a 
Coulomb Logarithm as the "Coulomb Log Approximation" (CLA). Thus, we say that under the CLA, our damping and diffusion coefficients (6.39) and (6.40) reduce to the Rosenbluth Potentials. By including the full spatial distribution in the formulation, we do not need to apply the CLA however, and our expressions have no large distance divergences, assuming the spatial distribution falls off appropriately fast at infinity. There is still the short range logarithmic divergence, however.

Let us now return to Eqs. (6.39) and (6.40) and plug them into Eq. (6.15) to find the second moment evolution due to IBS. Now, IBS will affect both the pure momentum moments and the mixed position-momentum moments of $d \bar{\Sigma}_{i j} / d t$. One can show that the mixed moments do not have the same logarithmic behavior as the pure momentum moments and we thus expect them to be smaller. A full analysis of this issue, however, should be considered further to give more confidence that this is always a good approximation. Thus, henceforth, we consider only $d \Sigma_{a b} / d t$ where the $a$ and $b$ represent momentum indices. In this case the growth rate can be expressed as

$$
\frac{1}{\tau_{a}}=\frac{1}{2\left\langle g_{a}\right\rangle} \operatorname{Tr}\left[\mathbb{C}_{a} \frac{d \bar{\Sigma}_{P}}{d t}\right]
$$

where $\bar{\Sigma}_{P}$ is the submatrix of momentum moments of $\Sigma$ with components $\bar{\Sigma}_{a b}$.

Substituting in the expressions (6.39) and (6.40), returning to $\vec{Z}_{2}$ as the integration variable, and combining the integrals together, we get

$$
\frac{d \bar{\Sigma}_{a b}}{d t}=\frac{4 N k^{2}}{m} \int \frac{d \vec{Z}_{1} d \vec{Z}_{2}}{\Delta^{2} r^{3}}\left[-\left(\hat{\Delta}_{a} P_{1 b}+\hat{\Delta}_{b} P_{1 a}\right)+\Delta \hat{r}_{a} \hat{r}_{b}\right] \bar{f}\left(\vec{Z}_{1}\right) \bar{f}\left(\vec{z}_{2}\right) \delta(\hat{\Delta} \cdot \hat{r})
$$

Next, consider what happens to the first term under a change of variables that interchanges 1 and 2 . The only changes will be that $\vec{P}_{1} \rightarrow \vec{P}_{2}$ and $\hat{\Delta} \rightarrow-\hat{\Delta}$. Thus under the integral we can make the substitution

$$
\hat{\Delta}_{a} P_{1 b} \rightarrow \frac{1}{2} \hat{\Delta}_{a}\left(P_{1 b}-P_{2 b}\right)=\frac{1}{2} \Delta \hat{\Delta}_{a} \hat{\Delta}_{b}
$$

which is the same for the term with $a \rightarrow b$ so that we can just multiply the first term 
by 2 . Now, factoring out the $\Delta$, and canceling one in the denominator, we have

$$
\frac{d \bar{\Sigma}_{a b}}{d t}=\frac{4 N k^{2}}{m} \int \frac{d \vec{Z}_{1} d \vec{Z}_{2}}{\Delta r^{3}}\left[\hat{r}_{a} \hat{r}_{b}-\hat{\Delta}_{a} \hat{\Delta}_{b}\right] \bar{f}\left(\vec{Z}_{1}\right) \bar{f}\left(\vec{Z}_{2}\right) \delta(\hat{\Delta} \cdot \hat{r})
$$

Taking a trace of this quantity gives 0 which shows that the average energy is conserved. Here we have computed the evolution of the pure momentum moments. The damping effect will also give rise to evolution of the mixed position momentum moments. Let us write $Z_{-b}=P_{b}$. We can show that

$$
\frac{d \bar{\Sigma}_{a-b}}{d t}=\frac{2 N k^{2}}{m} \int \frac{d \vec{Z}_{1} d \vec{Z}_{2}}{\Delta r^{2}}\left[\hat{r}_{a} \hat{\Delta}_{b}-\hat{r}_{b} \hat{\Delta}_{a}\right] \bar{f}\left(\vec{Z}_{1}\right) \bar{f}\left(\vec{Z}_{2}\right) \delta(\hat{\Delta} \cdot \hat{r})
$$

Note that there is one fewer power of $r$ in the denominator which will mean that there is no logarithmic behavior and leads us to consider these quantities as typically smaller than the pure momentum moment evolution. One interesting property one can see immediately from this expression is that it implies angular momentum conservation. For example

$$
\frac{d\left\langle L_{z}\right\rangle}{d t}=\frac{d \bar{\Sigma}_{x-y}}{d t}-\frac{d \bar{\Sigma}_{y-x}}{d t}=0
$$

and likewise for $\left\langle L_{x}\right\rangle$ and $\left\langle L_{y}\right\rangle$. This is independent of the beam distribution function $\bar{f}$.

Let us now assume that the distribution is a Gaussian function. We will further take it to be a function of the invariants of the linear one-turn map as we have discussed previously. For the distribution matrices, we write

$$
\overline{\mathbb{M}}=\left(\begin{array}{cc}
\mathbb{A} & \mathbb{B} \\
\mathbb{B}^{T} & \mathbb{C}
\end{array}\right)=\sum_{a=1}^{3} \overline{\mathbb{M}}_{a}=\sum_{a=1}^{3} \frac{1}{\epsilon_{a}}\left(\begin{array}{ll}
\mathbb{A}_{a} & \mathbb{B}_{a} \\
\mathbb{B}_{a}^{T} & \mathbb{C}_{a}
\end{array}\right)
$$

where $\overline{\mathbb{M}}=\mathcal{L}^{T} \mathbb{M} \mathcal{L}$ is $\mathbb{M}$ expressed in the beam frame and

$$
\overline{\mathbb{M}}_{a}=\frac{1}{\epsilon_{a}} \mathcal{L}^{T} \mathcal{B}^{T} G_{a} \mathcal{B} \mathcal{L}
$$

where we have transformed from betatron coordinates to physical coordinates. Eq. 
(6.52) becomes

$$
\frac{d \bar{\Sigma}_{a b}}{d t}=\frac{N k^{2}}{m \Gamma} \int \frac{d^{6} \eta}{\Delta r^{3}}\left[\hat{r}_{a} \hat{r}_{b}-\hat{\Delta}_{a} \hat{\Delta}_{b}\right] e^{-\frac{1}{2} \eta_{i} \eta_{j} \overline{\mathbb{M}}_{i j}} \delta(\hat{\Delta} \cdot \hat{r})
$$

Next, we consider spherical coordinates for both $\vec{\Delta}$ and $\vec{r}$. The delta function is going to do one of the angular integrals for us. We have a choice of which one. Define the following vectors:

$$
\hat{e}_{1}=\left(\begin{array}{c}
\sin \theta \cos \phi \\
\sin \theta \sin \phi \\
\cos \theta
\end{array}\right), \quad \hat{e}_{2}=\left(\begin{array}{c}
\cos \theta \cos \phi \\
\cos \theta \sin \phi \\
-\sin \theta
\end{array}\right), \quad \hat{e}_{3}=\left(\begin{array}{c}
-\sin \phi \\
\cos \phi \\
0
\end{array}\right)
$$

Then we can either let $\hat{\Delta}=\hat{e}_{1}$ and $\hat{b}=\cos \alpha \hat{e}_{2}+\sin \alpha \hat{e}_{3}$ or $\hat{b}=\hat{e}_{1}$ and $\hat{\Delta}=$ $\cos \alpha \hat{e}_{2}+\sin \alpha \hat{e}_{3}$.

Pulling out the constant term and writing

$$
\frac{d \bar{\Sigma}_{a b}}{d t}=\mathcal{A} \mathbb{K}_{a b}
$$

with

$$
\mathcal{A}=\frac{N r_{0}^{2} c}{32 \pi^{3} \beta^{3} \gamma^{4} \epsilon_{x} \epsilon_{y} \sigma_{s} \sigma_{\delta}}
$$

We have

$$
\mathbb{K}_{a b}=\int d \Delta d r d \Omega \frac{\Delta}{r} h_{a b} e^{-\frac{1}{2}\left(h_{1} r^{2}+h_{2} r \Delta+h_{3} \Delta^{2}\right)} \delta(\hat{\Delta} \cdot \hat{r})
$$

where

$$
h_{a b}=\left(\hat{r}_{a} \hat{r}_{b}-\hat{\Delta}_{a} \hat{\Delta}_{b}\right)
$$

and

$$
\begin{aligned}
h_{1} & =\overline{\mathbb{A}}_{a b} \hat{b}_{a} \hat{b}_{b} \\
h_{2} & =\overline{\mathbb{B}}_{a b} \hat{b}_{a} \hat{\Delta}_{b}+\overline{\mathbb{B}}_{a b} \hat{b}_{b} \hat{\Delta}_{a} \\
h_{3} & =\overline{\mathbb{C}}_{a b} \hat{\Delta}_{a} \hat{\Delta}_{b}
\end{aligned}
$$


Here,

$$
\begin{aligned}
& \overline{\mathbb{A}}=r_{m}^{2} \mathbb{A}, \\
& \overline{\mathbb{B}}=P_{0} r_{m} \mathbb{B}, \\
& \overline{\mathbb{C}}=P_{0}^{2} \mathbb{C}
\end{aligned}
$$

are unitless distribution matrices. We have changed variables to the unitless $r / r_{m}$ and $\Delta / P_{0}$ which is why these factors show up in the distribution matrices. Now, we do the $r$ and $\Delta$ integrals. We can see that the integral will diverge at $r=0$, and hence we need a minimum distance cut-off $r_{m}$. Because of the change of variables, we actually cut the $r$ integral off at 1 . As discussed, $r_{m}$ is $\Delta$ dependent. As an approximation, here, we will take $r_{m}$ to be a constant as noted earlier.

If we throw away the non-logarithmic terms, and ignore the factor of $\gamma_{E}-\ln 2$ (see appendix for derivation including these extra terms) we have:

$$
\mathbb{K}_{a b}=\int d \Omega \frac{h_{a b}}{h_{3}} \ln h_{1}
$$

We can reduce this to a double integral:

$$
\mathbb{K}_{a b}=-\int d \cos \theta d \phi \Theta_{a b}(\theta, \phi) \ln \left(h_{1}\right)
$$

where

$$
\Theta_{a b}(\theta, \phi)=\int_{0}^{2 \pi} \frac{h_{a b}}{h_{3}} d \alpha=2 \pi\left(\frac{\left(\hat{r}_{a} \hat{r}_{b}-\frac{1}{2} u_{a b}^{+}\right)}{\sqrt{q}}+\frac{1}{2} \frac{\left(\left(u_{a b}-w_{a b}\right)(a-c)+v_{a b} b\right)}{q+(a+c) \sqrt{q}}\right)
$$

with $u_{a b}^{+}=u_{a b}+w_{a b}$ and $u_{a b}^{-}=u_{a b}-w_{a b}$. For $u_{a b}, v_{a b}, w_{a b}$ we have explicitly

$$
\begin{aligned}
u_{a b} & =\hat{e}_{2 a} \hat{e}_{2 b} \\
v_{a b} & =\hat{e}_{2 a} \hat{e}_{3 b}+\hat{e}_{2 b} \hat{e}_{3 a} \\
w_{a b} & =\hat{e}_{3 a} \hat{e}_{3 b}
\end{aligned}
$$


and

$$
q=\frac{h_{2}}{\sqrt{h_{1} h_{3}-h_{2}^{2}}}
$$

The fact that $h_{1} h_{3}-h_{2}^{2}>0$ follows from the fact that $M_{i j}$ is positive definite, i.e. $M_{i j} z_{i} z_{j}>0$ for all $z_{i}$. This is our main result for the IBS emittance growth rates. We have not encountered any long distance divergence requiring a Coulomb Log. Indeed the spatial dependence of the beam is included in the matrix $\overline{\mathbb{A}}$, although we do note the factor of the minimum impact parameter in Eq. (6.66). We have reduced $\mathbb{K}$ to double angular integrals. These integrals can be done numerically and are not particularly time consuming.

In order to reproduce the results of Bjorken-Mtingwa, and further simplify the integration, we consider the Coulomb Log approximation for the moment evolution. This involves first doing the $r$ integral in (6.57) but ignoring the Gaussian function of $f$. This is done in the appendix. We could also combine Eqs. (6.46) and (6.47) directly in the moment evolution equation. In either case, the result is

$$
\mathbb{K}_{a b}^{\mathrm{BM}}=2 \pi^{2} \int_{0}^{\infty} d \lambda \frac{\sqrt{\lambda}}{\operatorname{det}(\Lambda)}\left(\delta_{a b} \operatorname{Tr}\left(\Lambda^{-1}\right)-3 \Lambda_{a b}^{-1}\right)
$$

where $\Lambda=\overline{\mathbb{C}}-\lambda I$. So, for the growth rate we get

$$
\frac{1}{\tau_{a}}=\frac{1}{2} \mathbb{C}_{a b}^{(a)} 2 L_{c}\left(2 \pi^{2}\right) \frac{N r_{0}^{2} c}{32 \pi^{3} \beta^{3} \gamma^{4} \epsilon_{x} \epsilon_{y} \sigma_{s} \sigma_{\delta}} \int_{0}^{\infty} d \lambda \frac{\sqrt{\lambda}}{\operatorname{det}(\Lambda)}\left(\delta_{a b} \operatorname{Tr}\left(\Lambda^{-1}\right)-3 \Lambda_{a b}^{-1}\right)
$$

Combined with explicit expressions for the submatrices of Eq. (6.55) for the uncoupled case, this reproduces the results of Bjorken-Mtingwa. We give these expressions in the next section. 


\subsection{Uncoupled Case}

Computing the uncoupled submatrices, we find

$$
\begin{aligned}
& \mathbb{A}_{x}=\left(\begin{array}{ccc}
\gamma_{x} & 0 & -\gamma \tilde{\mathcal{G}}_{x} \\
0 & 0 & 0 \\
-\gamma \tilde{\mathcal{G}}_{x} & 0 & 0
\end{array}\right) \\
& \mathbb{A}_{y}=\left(\begin{array}{ccc}
0 & 0 & 0 \\
0 & \gamma_{y} & -\gamma \tilde{\mathcal{G}}_{y} \\
0 & -\gamma \tilde{\mathcal{G}}_{y} & 0
\end{array}\right) \\
& \mathbb{A}_{z}=\left(\begin{array}{ccc}
\gamma_{z} \eta_{x}^{\prime 2} & \gamma_{z} \eta_{x}^{\prime} \eta_{y}^{\prime} & \gamma \alpha_{z} \eta_{x}^{\prime} \\
\gamma_{z} \eta_{x}^{\prime} \eta_{y}^{\prime} & \gamma_{z} \eta_{y}^{\prime 2} & \gamma \alpha_{z} \eta_{y}^{\prime} \\
\gamma \alpha_{z} \eta_{x}^{\prime} & \gamma \alpha_{z} \eta_{y}^{\prime} & \gamma^{2} \gamma_{z}
\end{array}\right) \\
& \mathbb{B}_{x}=\left(\begin{array}{ccc}
\alpha_{x} & 0 & 0 \\
0 & 0 & 0 \\
0 & 0 & 0
\end{array}\right) \\
& \mathbb{B}_{y}=\left(\begin{array}{ccc}
0 & 0 & 0 \\
0 & \alpha_{y} & 0 \\
0 & 0 & 0
\end{array}\right) \\
& \mathbb{B}_{z}=\left(\begin{array}{ccc}
-\gamma_{z} \eta_{x} \eta_{x}^{\prime} & -\gamma_{z} \eta_{x}^{\prime} \eta_{y} & \gamma \alpha_{z} \eta_{x}^{\prime} \\
-\gamma_{z} \eta_{x} \eta_{y}^{\prime} & -\gamma_{z} \eta_{y} \eta_{y}^{\prime} & \gamma \alpha_{z} \eta_{y}^{\prime} \\
-\gamma \gamma_{z} \eta_{x} & -\gamma \gamma_{z} \eta_{y} & \gamma^{2} \alpha_{z}
\end{array}\right) \\
& \mathbb{C}_{x}=\left(\begin{array}{ccc}
\beta_{x} & 0 & -\gamma \mathcal{G}_{x} \\
0 & 0 & 0 \\
-\gamma \mathcal{G}_{x} & 0 & \gamma^{2} \mathcal{H}_{x}
\end{array}\right) \\
& \mathbb{C}_{y}=\left(\begin{array}{ccc}
0 & 0 & 0 \\
0 & \beta_{y} & -\gamma \mathcal{G}_{y} \\
0 & -\gamma \mathcal{G}_{y} & \gamma^{2} \mathcal{H}_{y}
\end{array}\right)
\end{aligned}
$$




$$
\mathbb{C}_{z}=\left(\begin{array}{ccc}
\gamma_{z} \eta_{x}^{2} & \gamma_{z} \eta_{x} \eta_{y} & -\alpha_{z} \gamma \eta_{x} \\
\gamma_{z} \eta_{x} \eta_{y} & \gamma_{z} \eta_{y}^{2} & -\alpha_{z} \gamma \eta_{y} \\
-\alpha_{z} \gamma \eta_{x} & -\alpha_{z} \gamma \eta_{y} & \gamma^{2} \beta_{z}
\end{array}\right)
$$

We have introduced the definitions

$$
\begin{aligned}
& \mathcal{G}_{x, y}=\alpha_{x, y} \eta_{x, y}+\beta_{x, y} \eta_{x, y}^{\prime} \\
& \tilde{\mathcal{G}}_{x, y}=\gamma_{x, y} \eta_{x, y}+\alpha_{x, y} \eta_{x, y}^{\prime}
\end{aligned}
$$

Looking at the elements of $\mathbb{C}_{z}$, we find that the $\gamma^{2} \beta_{z}$ in the lower right corner will typically dominate, and we drop the other terms. Then,

$$
\mathbb{C}=\left(\begin{array}{ccc}
\frac{\beta_{x}}{\epsilon_{x}} & 0 & -\gamma \frac{\mathcal{G}_{x}}{\epsilon_{x}} \\
0 & \frac{\beta_{y}}{\epsilon_{y}} & -\gamma \frac{\mathcal{G}_{y}}{\epsilon_{y}} \\
-\gamma \frac{\mathcal{G}_{x}}{\epsilon_{x}} & -\gamma \frac{\mathcal{G}_{y}}{\epsilon_{y}} & \gamma^{2}\left(\frac{\beta_{z}}{\epsilon_{z}}+\frac{\mathcal{H}_{x}}{\epsilon_{x}}+\frac{\mathcal{H}_{y}}{\epsilon_{y}}\right)
\end{array}\right)
$$

For the uncoupled $\mathbb{A}$, if we just keep the $\gamma^{2}$ term in $\mathbb{A}_{z}$ we find

$$
\mathbb{A}=\left(\begin{array}{ccc}
\frac{\gamma_{x}}{\epsilon_{x}} & 0 & -\gamma \frac{\overline{\mathcal{G}}_{x}}{\epsilon_{x}} \\
0 & \frac{\gamma_{y}}{\epsilon_{y}} & -\gamma \frac{\mathcal{G}_{y}}{\epsilon_{y}} \\
-\gamma \frac{\mathcal{G}_{x}}{\epsilon_{x}} & -\gamma \frac{\mathcal{G}_{y}}{\epsilon_{y}} & \gamma^{2} \frac{\gamma_{z}}{\epsilon_{z}}
\end{array}\right)
$$

We now define the emittance growth rates as

$$
\frac{1}{\tau_{x, y, z}}=\frac{1}{\epsilon_{x, y, z}} \frac{d \epsilon_{x, y, z}}{d t}
$$

and we get

$$
\begin{aligned}
\frac{1}{\tau_{x}} & =\frac{\mathcal{A}}{\epsilon_{x}}\left(\beta_{x} \mathbb{K}_{x x}-2 \gamma \mathcal{G}_{x} \mathbb{K}_{x z}+\gamma^{2} \mathcal{H}_{x} \mathbb{K}_{z z}\right) \\
\frac{1}{\tau_{y}} & =\frac{\mathcal{A}}{\epsilon_{y}}\left(\beta_{y} \mathbb{K}_{y y}-2 \gamma \mathcal{G}_{y} \mathbb{K}_{y z}+\gamma^{2} \mathcal{H}_{y} \mathbb{K}_{z z}\right) \\
\frac{1}{\tau_{z}} & =\frac{\mathcal{A}}{\epsilon_{z}} \beta_{z} \mathbb{K}_{z z}
\end{aligned}
$$

We can now use our expression and that of the CLA to effectively define a Coulomb 
Logarithm for each of the moment growth equations:

$$
2 \log _{a b}=\frac{-\int d \Omega \frac{h_{a b}}{h_{3}} \log \left(\frac{h_{1}}{2}\right)}{\int d \Omega \frac{h_{a b}}{h_{3}}}=\frac{\mathbb{K}_{a b}}{\mathbb{K}_{a b}^{\mathrm{BM}}}
$$

Note that this actually defines 9 Coulomb Logs: one for each of the growth rates of $\left\langle P_{a} P_{b}\right\rangle$. If all of the Coulomb Logs so calculated come out similarly, then one can use the CLA with an appropriate Coulomb Log. However, if the different $\log _{a b}$ come out substantially differently, then the CLA is not applicable.

Let us consider the calculation of $\log _{a b}$ for a real storage ring. We take the case of the ATF at KEK. The parameters are given in Table 6.1. The ATF was designed as a test facility for damping rings for a high energy linear collider. It can produce beams with some of the smallest emittances ever achieved. Further, as a result of minimizing vertical emittance and coupling, the vertical emittance is extremely small. It is in such a situation that one may question whether the CLA is valid. Even if one Coulomb log is appropriate, what is its value? What should one use for the maximum impact parameter? The three beam sizes, $\sigma_{x, y, z}$ provide substantially different length scales.

Now, because of radiation damping and diffusion, as was discussed in chapter 4, the beam will reach an equilibrium. IBS will change this equilibrium. We discuss the combination of IBS and synchrotron radiation (SR) more in the next chapter. For now, we note that we have written a program to evolve the emittances in the presence of both IBS and SR. After equilibrium was reached, we computed the ratio of the Coulomb Log defined in (6.93) and the Coulomb Log determined by using the vertical beam size as $b_{\max }$. We vary the vertical dispersion which determines the non-IBS vertical emittance through the quantity $\mathcal{H}_{y}$. Thus, varying $\eta_{y}$ is essentially varying the beam aspect ratio. We use a smoothed version of the ATF with parameters given in Table 6.1.

In the ATF, the beam is coolest in $\delta$ and hottest in $x$. Thus, $\mathbb{K}_{33}$ is positive and $\mathbb{K}_{11}$ negative. $\mathbb{K}_{22}$ can be either positive or negative (energy conservation gives $\sum_{a} \mathbb{K}_{a a}=0$.) We find the biggest difference between $\mathbb{K}_{a b}$ and $\mathbb{K}_{a b}^{\mathrm{BM}}$ for $\mathbb{K}_{22}$. In the small coupling limit, the $\mathbb{K}_{22}$ and the $\mathbb{K}_{33}$ contributions to $1 / T_{2}$ have a relative 
Table 6.1: ATF, RHIC Parameters

\begin{tabular}{|ccc|}
\hline parameter & ATF & RHIC (AU) injection \\
\hline \hline $\mathrm{C}$ & $138.6 \mathrm{~m}$ & $3833.845 \mathrm{~m}$ \\
\hline $\mathrm{N}$ & $9 \times 10^{9}$ & $1.3 \times 10^{9}$ \\
\hline $\mathrm{m}$ & $m_{e}$ & $197 m_{n}$ \\
\hline $\mathrm{Z}$ & -1 & 79 \\
\hline$E_{0}$ & $1.28 \mathrm{GeV}$ & $9.8 \mathrm{GeV} / \mathrm{u}$ \\
\hline$\gamma$ & 2505 & 10.5 \\
\hline$\nu_{s}$ & 0.0049 & 0.007 \\
\hline$\alpha_{c}$ & 0.0029 & 0.0016 \\
\hline$\epsilon_{x}$ & $1.05 \times 10^{-9} \mathrm{~m}$ & $2.4 \times 10^{-7}$ \\
\hline$\epsilon_{y}$ & $5 \times 10^{-12} \mathrm{~m}$ & $2.4 \times 10^{-7}$ \\
\hline$\epsilon_{z}$ & $2.81 \times 10^{-6} \mathrm{~m}$ & $1.1 \times 10^{-3}$ \\
\hline$\chi_{x}$ & $2.5 \times 10^{-5}$ \\
\hline$\chi_{y}$ & $1.6 \times 10^{-5}$ \\
\hline$\chi_{z}$ & $2.2 \times 10^{-5}$ \\
\hline$d_{x}$ & $10.5 \times 10^{-13} \mathrm{~m}$ \\
\hline$d_{z}$ & $2.46 \times 10^{-10} \mathrm{~m}$ \\
\hline
\end{tabular}


coefficient of $\gamma^{2} \eta_{y}^{2} / \beta_{y}^{2}$. In the "High Energy Approximation", one keeps only the $\mathbb{K}_{33}$ term. However, for the ATF parameters, if $\eta_{y}<1.8 \mathrm{~mm}, \mathbb{K}_{22}$ can become important. In Figure 6.2 we plot the ratio of the Coulomb logs defined in (6.93) to the conventional Coulomb $\log L_{c}=\log \left(\sigma_{y} / r_{m}\right)\left(L_{c} \approx 16\right.$ for ATF) for varying vertical dispersion and zero coupling (since BM dealt only with the uncoupled case). As stated, we are varying the beam aspect ratio, with the right side of the plot approaching a round beam. Near $\eta_{y}=28 \mathrm{~mm}$, the intrinsic vertical growth rates $\mathbb{K}_{22}$ and $\mathbb{K}_{22}^{\mathrm{BM}}$ have opposite signs. Finally note that both $\log _{11} / L_{c}$ and $\log _{33} / L_{c}$ are close to 1 over a wide range of beam aspect ratios. See [18] for more details.

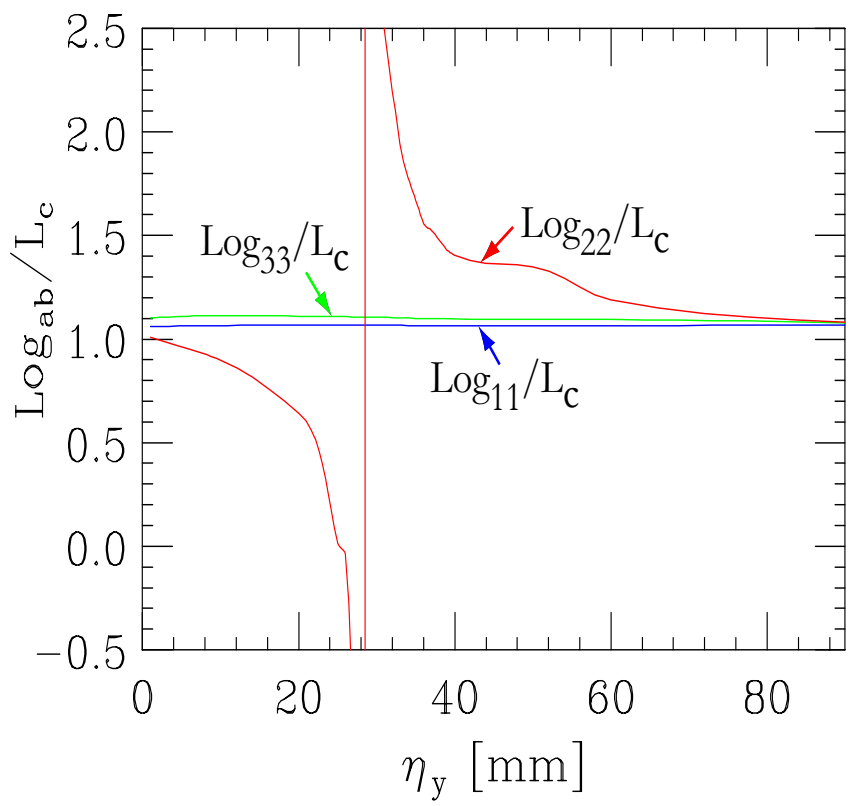

Figure 6.1: Coulomb Logs for ATF

Figure 6.2 shows that the Coulomb Log approximation is excellent except for the growth rates of the momentum second moments $\left\langle P_{x}^{2}\right\rangle$ and $\left\langle P_{z}^{2}\right\rangle$ and not particularly good for $\left\langle P_{y}^{2}\right\rangle$ over this parameter range. The range where the CLA for the vertical growth rate breaks down is where the vertical growth rate goes to zero. Normally the longitudinal temperature is much smaller than transverse, but because of the very small vertical emittance for the ATF, the vertical growth rate can go to zero or even become negative. Even though the CLA can break down, there is very little practical consequence to this for this parameter range. This is because coupling or 
vertical dispersion cause the vertical emittance growth rate to be dominated still by the longitudinal growth which is not going to zero and where CLA applies.

In general, near where one of the the moment growth rates goes to zero is where we expect to find differences between our expression and that of the CLA. As another example, we consider the case of RHIC, comparing data to analytic formulas. The parameters are given in Table 6.1 and the data is drawn from [72]. Below transition there is approximately an equilibrium. The equilibrium occurs approximately when the beam-frame momentum moments are equal. For the uncoupled case, and ignoring dispersion, this occurs when

$$
\frac{\sigma_{\delta}}{\gamma}=\sqrt{\frac{\epsilon_{x}}{\beta_{x}}}=\sqrt{\frac{\epsilon_{y}}{\beta_{y}}}
$$

RHIC has a round beam and thus the second two of these quantities are approximately equal. The ratio of the first to the second is found to be 0.7 , so the beam is indeed near an IBS equilibrium.

Near equilibrium, we find that our results differ from those of B-M. We find a small, but possibly measurable difference and our equations give results closer to the data than those of B-M. This comparison is shown in Figure 6.2. The data is RMS bunch length data measured with a wall current monitor. More details of the measurements and conditions are given in the referenced paper. One should note that for the transverse dynamics, additional growth was found that was not likely to be IBS. We find that this additional growth does not have much effect on the longitudinal growth.

Another common formulation of IBS theory used is that of Piwinski. It is essentially the same as that of Bjorken and Mtingwa, except for retaining the $\Delta$ dependance of $r_{\min }$. There are many ways of approximating Bjorken-Mtingwa and Piwinski and much of the recent literature on IBS involves finding approximations which are valid in various useful limiting cases. This is useful so that one can evaluate the integrals rapidly and average around a full lattice.

A variety of approximations to the Bjorken-Mtingwa result exist. For example, Lebedev et. al. considers the limit where $\sigma_{p_{z}} \ll \sigma_{p_{x, y}}$ Here, this means $\mathbb{C}_{33} \gg \mathbb{C}_{11,22}$. 


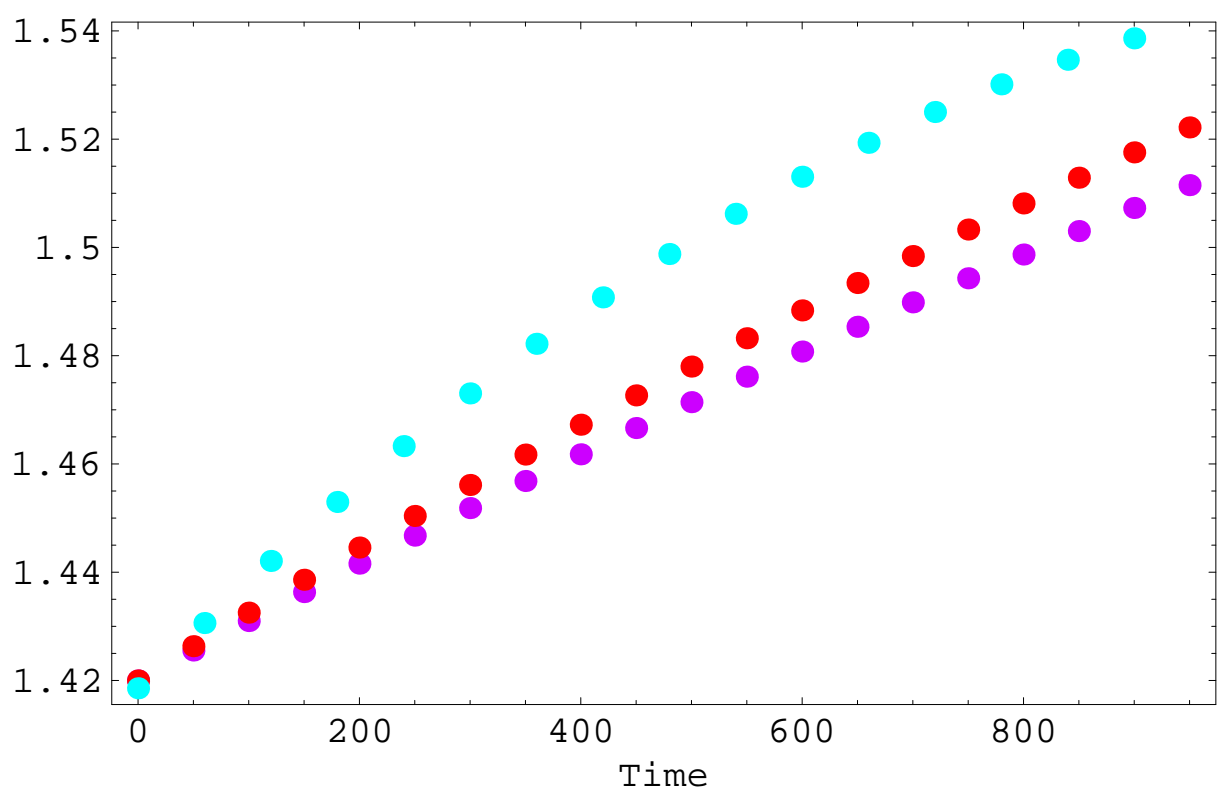

Figure 6.2: Comparison of B-M to our formulae in reproducing RHIC AU bunch length data $[72,73]$ The vertical axis is RMS bunch length measured from a wall current monitor, and time is measured in seconds. The blue points represent the bunch length data. The red points give bunch length evolution using our equations which we computed using Eq. (6.70) and the purple dots represent the results of Bjorken-Mtingwa, Eq. (6.74). 
In this limit we can show

$$
\mathbb{K}^{\mathrm{BM}}{ }_{a b} \approx \frac{\pi}{\sqrt{\mathbb{C}_{33}}} \int_{0}^{2 \pi} d \phi \frac{1}{\sqrt{\mathbb{C}_{11} \sin ^{2} \phi+\mathbb{C}_{22} \cos ^{2} \phi}}
$$

Note that when we put the prefactor back in for the growth rate, the dependence on $\sigma_{\delta}$ drops out. This can be interpreted to mean that as far as the scattering process is concerned, in this limit, one can assume no initial relative longitudinal motion.

\subsection{Equilibrium distributions}

Intrabeam scattering gives us a specific model of thermal equilibration. We might ask therefore about what kinds of equilibria can be achieved. From a general perspective, one might expect a distribution of the form $e^{-P^{2} / 2 m K T}$ where $K$ is Boltzmann's constant, and $T$, some effective temperature. We find that for the CLA, this is true, but our expressions give a small deviation from the Boltzman distribution. Here we consider the case of a round beam $\left(\mathbb{A}_{11}=\mathbb{A}_{22}=A, \mathbb{C}_{11}=\mathbb{C}_{22}=C\right)$ and try to find equilibria. Within the Gaussian approximation, this means $\mathbb{K}_{a b}=0$. Using symmetry and energy conservation, we have that $\mathbb{K}_{11}=\mathbb{K}_{22}=-\frac{1}{2} \mathbb{K}_{33}$. Thus, all we need for equilibria is that $\mathbb{K}_{33}=0$. Because of the symmetry, $\mathbb{K}_{33}$ is a function of 3 parameters. The parameters we use are

$$
\begin{aligned}
\tilde{A} & =\frac{A}{\mathbb{A}_{33}}-1 \\
\tilde{C} & =\frac{C}{\mathbb{C}_{33}}-1 \\
L_{c} & =\frac{1}{2} \log A
\end{aligned}
$$

Details of the calculation are shown in the appendix and the results are shown in figures (6.3) and (6.4).

When $\tilde{C}=0$ we have the Boltzman distribution. For Bjorken-Mtingwa, if $\tilde{C}=0$, it is always an equilibrium. For our expressions for $\mathbb{K}_{a b}$ we find that if $\tilde{C}=0$, we also need $\tilde{A}=0$, i.e. a spherical beam in position space. What is perhaps surprising is 
that we find that for $\tilde{C} \neq 0$, we can also find equilibria, although as we increase $L_{c}$, $\tilde{C}$ must be smaller and smaller for there to still be an equilibrium.

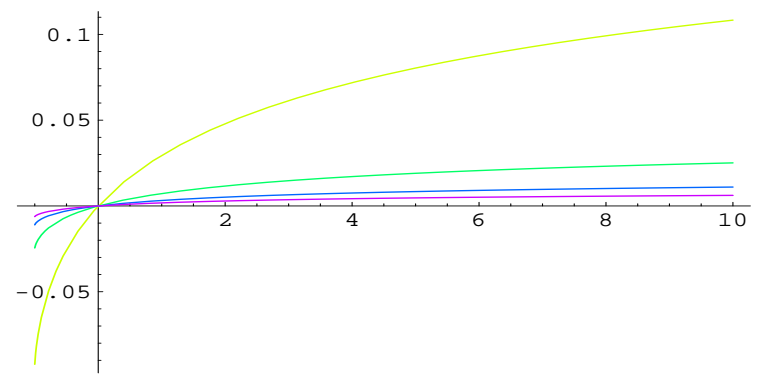

Figure 6.3: Equilibrium value of $\tilde{C}$ as a function of $\tilde{A}$ for several different values of $L_{c}=\frac{1}{2} \log A$ ("Coulomb Log"). $L_{c}=5$ is yellow, $L_{c}=10$ is green, $L_{c}=15$ is blue, and $L_{c}=20$ is purple.

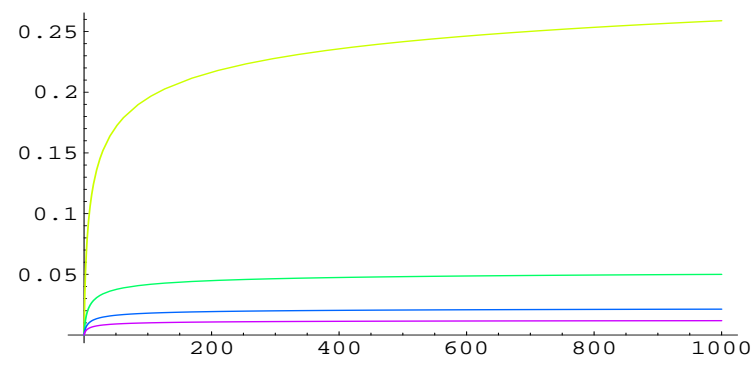

Figure 6.4: Same as previous figure with larger range of $\tilde{A}$.

Now we consider the question of equilibrium in the Coulomb Log approximation. At a given position, using the Coulomb-Log approximation, the distribution is in equilibrium if

$$
\left\langle x^{\prime 2}\right\rangle=\left\langle y^{\prime 2}\right\rangle=\frac{\left\langle\delta^{2}\right\rangle}{\gamma^{2}}
$$

We first consider the case of the smooth approximation.

We can see that above transition, in the smooth approximation, there is no equilibrium due to IBS alone. How does this picture change when we go beyond the smooth approximation? Following Bjorken-Mtingwa, we compute the sum of the growth rates and find it is strictly positive. Thus, except in the smooth approximation, there is not equilibrium.

In the case where $\epsilon_{x}=\epsilon_{y}=\epsilon_{\perp}$ and $\beta_{x}=\beta_{y}=\beta_{\perp}$, we find a total growth rate at 
any position of

$$
\frac{1}{\tau}=2\left(\frac{\beta_{\perp}}{\epsilon_{\perp}}-\frac{\beta_{z}}{\epsilon_{z}}\right)
$$

This quantity is always greater than or equal to 0 and hence as long as $\beta_{\perp}$ is varying, as it will in any strong focussing machine, there can be no equilibrium due to intrabeam scattering, even below transition! The paper of B-M contains a general expression for the total growth rate in terms of the eigenvalues of the matrix $\mathbb{C}$. In particular, they find ([13] Eq. 3.6) that the total growth rate is given by (in our notation)

$$
\frac{1}{\tau}=\mathcal{A}\left(\lambda_{1}-\lambda_{2}\right)^{2} \int_{0}^{\infty} \frac{d \lambda \sqrt{\lambda}}{\left(\lambda_{1}+\lambda\right)^{\frac{3}{2}}\left(\lambda_{2}+\lambda\right)^{\frac{3}{2}}\left(\lambda_{3}+\lambda\right)^{\frac{1}{2}}}+\text { two cyclic permutations }
$$

where $\Lambda=\mathbb{C}+\lambda I$ and $\lambda_{1,2,3}$ are the eigenvalues of $\mathbb{C}$. They compute the integrated growth rate, but without integrating around the ring, this expression gives the local growth rate as well. This quantity is clearly always positive. Thus, for a non-smooth lattice, there can be no equilibrium. Thus, the conclusion we given here for a special case is actually quite general.

\subsection{Overview of IBS Literature}

We have developed our own approach to IBS in this thesis. We have seen that if we make what we call the "Coulomb Log Approximation", our expressions for the growth of the second moments reduce to the expressions given by Bjorken-Mtingwa.

The other main approach to IBS is that of Piwinski[12] who is the original author to tackle this subject in the context of beams in storage rings. Although the starting point is different, his work can be understood as the same in effect as Bjorken Mtingwa, except for the minimum impact parameters dependence on the momentum of the two particles scattering is retained in the integral instead of replacing it with a constant average value allowing one to pull out a Coulomb Log from the integral. Thus, from this perspective, his equations are indeed more exact than Bjorken-Mtingwa's. Our approach provide a branching off point from which one could derive either Piwinski or B-M's results. The common ground is contained in Eq. (6.57). 
The original paper of Piwinski's did not include general Courant-Snyder lattice parameters or the derivative of the dispersion function. His work was generalized to include these.

Bjorken-Mitingwa approach the subject from a quantum mechanical scattering formalism, but taking lowest order and given the result that the classical and quantum scattering cross section for the Coulomb interaction agree to this order, the result is the same as the classical analysis. Once this fact is recognised, there are two remaining differences between the two analyses. The first is that Piwinski does not include the full variation of the lattice parameters in his analysis, whereas BjorkenMtingwa do. This was remedied in later work, by Martini, Piwinski and others. The other difference relates to the issue of the Coulomb Logarithm. In computing the growth rate due to scattering, one must integrate over impact parameters. In the formalism used by Bjorken-Mtingwa and Piwinski this integrals diverges logarithmically at both small and large impact parameters requiring the introduction of cut-offs $b_{\min }$ and $b_{\max }$. Both analyses retain an undefined maximum distance cut-off related to the size of the beam. For the minimum distance cut-off one uses the distance of minimum approach of the particles. This depends on the relative momenta of the two scattering particles. Piwinski retains this momentum dependence of $b_{\text {min }}$ whereas Bjorken-Mtingwa replace it with an average value. Thus, once the variation of the lattice parameters is included, Piwinski's is the more accurate equation, although the paper's of Bjorken-Mtingwa and subsequent approximations and elaborations of both authors include many insights on the subject.

There are also a variety of approximations to B-M and Piwinski. See for example, references $[45,46,47,48,49,53,61]$ and references therein. For some early history of IBS, see [54].

\subsection{Beam-Beam Diffusion}

Our work can be applied to the beam beam interaction. Here we find the diffusion and damping coefficients and resulting moment evolution due to the beam-beam interaction in a collider. A calculation of such an effect was given in [74] under 
the description "beam-beam diffusion model". The diffusion coefficient is computed roughly along with the resulting emittance growth. Using the formalism described in this thesis, one could do a more careful computation. We sketch how this could be done.

We start by considering the scattering of two particles at phase space positions $\vec{z}_{1}$ and $\vec{z}_{2}$. The fields due to a particle with large $\gamma$ in the lab frame are given by

$$
E_{r}=B_{\theta}=\frac{2 q}{r} \delta(s-c t)
$$

The resulting force causes an increase in transverse slope of the particle's orbit determined by the equation

$$
y^{\prime \prime}=\frac{F_{y}}{m \gamma \beta^{2} c^{2}}
$$

for the case where the force is in the $y$ direction. This leads to a kick in transverse momentum

$$
\delta \frac{\vec{p}_{\perp}}{P_{0}}=\frac{4 q^{2}}{\gamma m \beta^{2} c^{2}} \frac{\hat{r}}{r} \equiv k \frac{\hat{r}}{r}
$$

where $\vec{r}=r \hat{r}=\vec{x}_{1}-\vec{x}_{2}$ is the relative position of the two particles at the time of collision (transverse). Given this result, we could now compute the diffusion and damping coefficients. Due to the reversibility condition, we can see that the damping coefficients are zero.

We note that the transverse momentum kick depends only on the transverse separation of the particles. Thus, the probability distribution $g(\Phi)$ that we must average over, will be the transverse particle distribution at the given location of the particle. For the quantity $\dot{n}$, we again take

$$
\dot{n}=N \beta c \int d \vec{x}_{\perp} \rho\left(\vec{x}_{\perp} ; z\right)
$$

where

$$
\rho(\vec{x})=\int d \vec{p} f(\vec{x}, \vec{p})
$$

is the spatial distribution of the beam. $z$ is the longitudinal position of the particle in the oncoming bunch. We could write the distribution of colliding particles explicitly 
as

$$
g\left(\vec{x}_{\perp}\right)=\frac{\rho\left(\vec{x}_{\perp} ; z\right)}{\int d \vec{x}_{\perp} \rho\left(\vec{x}_{\perp} ; z\right)}
$$




\section{Chapter 7}

\section{Coupled IBS and Combination with SR}

In this chapter, we apply our results on coupled invariants to IBS.

\subsection{Coupled Matrices}

The expressions for the emittance growth rates due to IBS for Gaussian beams was expressed in terms of the beam distribution in the beam frame. The position distribution was represented by the matrices $\mathbb{A}_{a}$ and the momentum distribution by $\mathbb{C}_{a}$ with the mixed components given $\mathbb{B}$. When the Coulomb Log approximation is made, only the $\mathbb{C}_{a}$ are involved, $\mathbb{A}$ essentially being absorbed into a Coulomb Log. In this section we will make the Coulomb Log approximation and write down the coupled $\mathbb{C}_{a}$; the coupled $\mathbb{A}_{a}$ can be written down in an analogous manner.

First consider an $x-y$ difference resonance, with a coupling angle $\theta$. We find

$$
\begin{aligned}
& \mathbb{C}_{1}=\cosh ^{2}\left(\frac{\theta}{2}\right) \mathbb{C}_{x}+\sinh ^{2}\left(\frac{\theta}{2}\right) \mathbb{C}_{y}+\sinh (\theta) \mathbb{C}_{c}^{+}, \\
& \mathbb{C}_{2}=\sinh ^{2}\left(\frac{\theta}{2}\right) \mathbb{C}_{x}+\cosh ^{2}\left(\frac{\theta}{2}\right) \mathbb{C}_{y}+\sinh (\theta) \mathbb{C}_{c}^{+},
\end{aligned}
$$


for the sum resonance and

$$
\begin{aligned}
\mathbb{C}_{1} & =\cos ^{2}\left(\frac{\theta}{2}\right) \mathbb{C}_{x}+\sin ^{2}\left(\frac{\theta}{2}\right) \mathbb{C}_{y}+\sin (\theta) \mathbb{C}_{c}^{-} \\
\mathbb{C}_{2} & =\sin ^{2}\left(\frac{\theta}{2}\right) \mathbb{C}_{x}+\cos ^{2}\left(\frac{\theta}{2}\right) \mathbb{C}_{y}+\sin (\theta) \mathbb{C}_{c}^{-}
\end{aligned}
$$

for the difference resonance. The extra terms are given by

$$
\mathbb{C}_{c}^{+}=\left(\begin{array}{ccc}
0 & \sqrt{\beta_{x} \beta_{y}} \cos \phi & 0 \\
\sqrt{\beta_{x} \beta_{y}} \cos \phi & 0 & 0 \\
0 & 0 & 0
\end{array}\right)
$$

and

$$
\mathbb{C}_{c}^{-}=\left(\begin{array}{ccc}
0 & -\sqrt{\beta_{x} \beta_{y}} \sin \phi & 0 \\
-\sqrt{\beta_{x} \beta_{y}} \sin \phi & 0 & 0 \\
0 & 0 & 0
\end{array}\right)
$$

Now, we recall that the matrix $\mathbb{C}$ is required for the moment evolution matrix $\mathbb{K}$. The $\mathbb{C}_{a}$ are then needed to compute the growth of the invariants as we see from Eq. (6.49).

\subsection{Coupled Emittance Evolution and Combina- tion with Synchrotron Radiation}

To combine the effects of IBS and synchrotron radiation, one simply computes the emittance growth due to each effect per turn and add them together. The total change per turn is given by

$$
\Delta\left\langle g_{a}\right\rangle=-2 \chi_{a}\left\langle g_{a}\right\rangle+\bar{d}_{a}+\left\langle g_{a}\right\rangle \frac{\tau_{a}}{T_{0}}
$$

where $T_{0}$ is the revolution time.

Next, we return the example of the ATF at KEK to illustrate the inclusion of IBS and SR damping/diffusion. We also include global $x-y$ coupling of 5 degrees. This coupling angle was chosen to best fit equilibrium measured emittances. This coupling has a substantial effect on the projected vertical emittance because of the small beam 
aspect ratio. To see this, note the difference between emittance of eigenmode 2 and the vertical projected emittance. Also, we see the ratio of the projected vertical emittance to the transverse emittance does not stay constant over time, although the change is not extreme. This reminds us that the approach to IBS in which projected vertical emittance is simply taken as a multiplicative factor times the horizontal emittance is not exact.

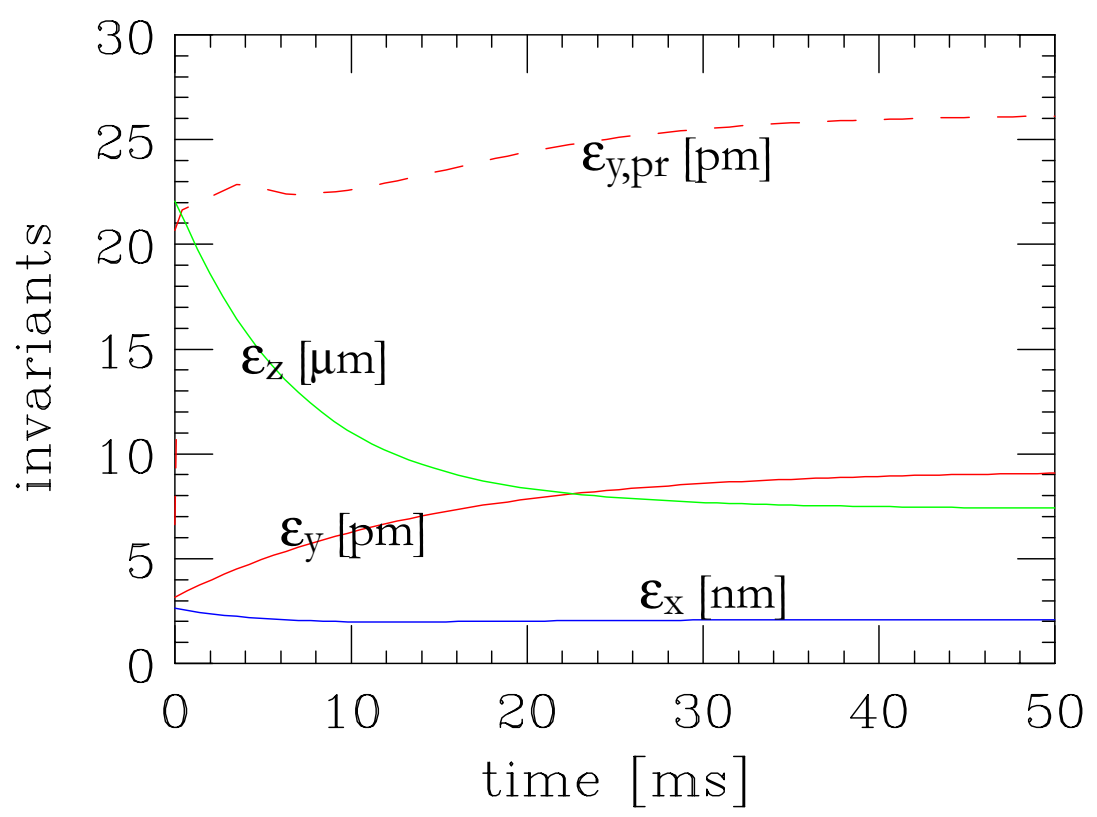

Figure 7.1: Evolution of Coupled Emittances for ATF

We include this plot to show the type of evolution that can occur for the emittances as they approach equilibrium due to both IBS and synchrotron radiation. More details and discussion are given in [18].

\subsection{Coupled Piwinski Invariant}

Without coupling, in the smooth approximation, the Piwinski invariant $[12,56,57]$ expresses conservation of energy in terms of the invariants transformed into the beam frame. It is given by

$$
\frac{\left\langle g_{x}\right\rangle}{\beta_{x}}+\frac{\left\langle g_{y}\right\rangle}{\beta_{y}}+\eta_{s} \frac{\left\langle g_{z}\right\rangle}{\beta_{z}}=\text { invariant }
$$


where $\eta_{s}=\frac{1}{\gamma^{2}}-\alpha_{c}$ is the slip factor. Above transisition $\eta_{s}<0$ and the coefficients of the first and third term have opposite signs which implies that there is no equilibrium. Below transition $\eta_{s}>0$ which gives the same sign for all terms in the invariant which implies an equilibrium. Now, consider synchrobetatron coupling with a coupling angle of $\theta$ near a sum or difference resonance. If we ignore the extra term $G_{c}$, then expressing the Piwinski invariant in terms of the coupled $g_{1}$ and $g_{3}$, we find an invariant

$$
\left[\frac{c^{2}}{\beta_{x}}-\frac{s^{2} \eta_{s}}{\beta_{z}}\right]\left\langle g_{1}\right\rangle+\left[\frac{c^{2} \eta_{s}}{\beta_{z}}-\frac{s^{2}}{\beta_{x}}\right]\left\langle g_{3}\right\rangle+\frac{\left\langle g_{y}\right\rangle}{\beta_{y}}=\text { invariant }
$$

where for the difference resonance, $c^{2}=\cos ^{2}(\theta / 2)$ and $s^{2}=\sin ^{2}(\theta / 2)$ and for the sum resonance $c^{2}=\cosh ^{2}(\theta / 2)$ and $s^{2}=\sinh ^{2}(\theta / 2)$. Now, consider the case of small, positive $\eta_{s}$. This is below transition and stable in the uncoupled case. We can easily see that for large enough $\theta$, the second term will be negative and the first term positive. Thus, the synchrobetatron coupling extends the region for which there is no IBS instability to below transition. If we expand in small $\theta$, we find that there is no IBS equilibrium when

$$
\frac{\theta}{2}>\sqrt{\frac{\eta_{s} \beta_{x}}{\beta_{z}}} \approx \sqrt{\frac{\beta_{x} \mu_{s}}{C}}
$$

where we used the fact that $\beta_{z} \approx C\left|\eta_{s}\right| / \mu_{s}$. This is true for both the sum and difference resonance. For example, in the case of coupling due to dispersion at an RF cavity, we find that there is no IBS equilibrium below transition when

$$
\eta>\frac{2 \Delta \nu \sqrt{\eta_{s}}}{\nu_{s}} \beta_{x}
$$

where $\eta$ is the dispersion at the RF cavity and $\Delta \nu$ is the tune split between betatron and synchrotron tune. We have assumed that the coupling angle is small in this derivation.

Finally, we note that we have been using the smooth approximation in this discussion. If we drop this condition, the earlier discussion applies and there is generally no equilibrium, even well below transition. 


\section{Chapter 8}

\section{Conclusions and Future Work}

In this dissertation, we have considered a general framework for computing emittance evolution due to linear dynamics in storage rings with additional diffusive/dissipative processes. We find that the emphasis on global invariants and local stochastic moment evolution helps clarify the structure of this important element of storage ring physics.

Regarding the invariants, we have considered the case of weak coupling near a linear resonance and formulated a perturbation theory to get analytical expressions. The key to the perturbation theory was to start with the one-turn map exactly on resonance and consider both difference from resonance and coupling as perturbations. The map exactly on resonance has at least two equal eigenvalues, and so we require degenerate perturbation theory. Defining appropriate "co-vectors" we found that the results are expressed in terms of matrix elements of the perturbation and is isomorphic to perturbation theory in time-independent quantum mechanics. For a storage ring, the matrix is symplectic whereas in quantum mechanics the matrix is Hermitian. The difference between the formalisms shows up in the symmetry properties of the matrix elements. In the symplectic case, the eigenvectors are either a positive or negative mode. Whether or not the two degenerate modes have the same or different sign account for either stability or instability in the perturbed eigentunes. For the case of the integer and half-integer resonances, we find that in order to see the instability due to coupling, we need to do second order perturbation theory. Besides 
doing this just for the sake of completeness, we find that one of our motivating applications require this: an issue of current importance is the synchrobetatron coupling resonances induced by adding a crab cravity to a storage ring. We are particularly interested in the case of the horizontal tune near a half integer as this is a typical operating condition for B-factory colliders such as KEK-B and PEP-II. In addition, because the synchrotron tune is always small, we find that we must consider the effect of coupling on the integer $\nu_{z}$ resonance. Indeed, for $\nu_{x}>1 / 2$, we find that the crab cavity can cause an instability and emittance growth as well. For our other example of synchrobetatron coupling, dispersion at a crab cavity, we found that there was no instability at the $\nu_{z}=0$ resonance. This is perhaps fortunate, because of the ubiquity of this type of perturbation in storage rings.

We saw that the emittance evolution equations were particularly simple for the case of uniform damping and diffusion as is the case for synchrotron radiation. The invariants and damping and diffusion coefficients are combined together into damping decrements and global diffusion coefficients for the eigenmodes. We have explored behavior of each of these quantities near linear resonances. Near the difference resonance (the one stable resonance), we find a mixing of the uncoupled damping and diffusion coefficients. For the case of synchrobetatron coupling, the transverse direction is most strongly affected because the transverse diffusion and emittances are typically much smaller than the longitudinal. Near the unstable resonances (sum, integer, half-integer), the diffusion coefficients are mixed but also diverge at the resonance. At the sum resonance, we find that one of the damping decrements becomes negative indicating an anti-damping instability. Near the integer and half-integer resonances, the damping decrements are not affected much.

When the damping and diffusion depend on the phase space position, we need to directly compute the local moment evolution from the damping and diffusion rather than finding global damping/diffusion as we did in the uniform case. In actuality, non-uniform damping/diffusion will lead to non-Gaussian beams. Analysis of this issue requires solving the Fokker-Planck equation which we don't consider in this thesis.

Intrabeam scattering is our main example of a non-uniform stochastic process. 
However, we have formulated our stochastic approach such that it is computed in a way directly analogous to the uniform case of synchrotron radiation. We compute the momentum change per scatter with another particle in the beam and add up the totality of such scatters. By defining the scatter to occur at the distance of closest approach, we are able to partition the beam into a region that will undergo scatters within $\Delta t$ and the rest that will not.

This derivation is purely classical and the assumptions are relatively clear. An additional benefit we find from our formulation is that there is no large distance divergence in the integration over other particles that leads to a Coulomb logarithm $L_{c}$ in standard approaches. Such approaches are typically claimed to be valid to an accuracy of order $1 / L_{c}$. We hope that our formulation can improve this accuracy. Although we have improved on the standard approaches, we have also made a number of approximations for ease of calculation. One such approximation is in neglecting the evolution of the mixed position-momentum moments. We have also neglected the relative momentum dependence of the minimum distance in the integration. To be very clear about the accuracy of our expressions, the validity of these approximations should be further explored. Regarding the latter approximation involving the minimum distance, Bjorken-Mtingwa make the same approximation. Piwinski does not make this approximation, however, and in this respect is more accurate than B-M. For a fully well-defined approach, one should keep this dependence as Piwinski does. This can be done by performing the integration in a different order, but the full exploration of this issue is a topic for future research. We thus characterize our result as more precise than B-M, but making a different approximation than Piwinski, which may have relevance at lower energies. We have done all our comparison to B-M, which is generally seen to give adequate prediction of data in the studies thus far performed. We have shown an example, based on some measurements at RHIC where our formulae can plausibly be said to give more precise results than B-M. We have seen that this will tend to occur in the region where one of the momentum growth rates goes to zero. In flat beams such as in the ATF at KEK or the ALS, the growth rates of $\left\langle y^{\prime 2}\right\rangle$ can vanish for reasonable parameters, but the vertical emittance growth rate $1 / \tau_{y}$ is typically dominated by the growth of $\left\langle\delta^{2}\right\rangle$ due to vertical dispersion or $\mathrm{x}-\mathrm{y}$ coupling. 
Once one has expressions for the moment growth equations, they can be combined with the expressions for the invariants to find the eigen-emittance growth equations. Using our explicit expressions for coupled invariants near resonances, we can thus find IBS growth rates near such resonances. In addition to needing the coupled invariants to determine the definition of the emittances, one also needs the invariants to use in the beam distribution formulae which enter into the IBS moment growth equations. Thus, we can write down explicit expressions for IBS growth rates including coupling. This has been done in generality, but without providing explicit expressions. We expect that using explicit expressions for the coupled invariants and distribution can help in understanding IBS effects in a variety of coupled scenarios.

When IBS is combined with synchrotron radiation (SR), an equilibrium clearly exists, typically not too far from the equilibrium due to SR alone. When IBS is the only effect, the issue of equilibrium is a bit more subtle. In the Coulomb Log approximation (such as B-M), only the momentum distribution is involved with moment growth due to IBS. The result is basically that the beam tends to the Boltzman distribution if it can be reached. In our formulation involving the spatial distribution and no Coulomb Log, we find equilibrium that varies by a small amount from the Boltzman distribution. Assuming one can fully justify the use of the scattering approach to IBS, our result gives information on the locality of IBS. It suggests that the full beam size is relevant to IBS, but only to a small extent. This issue of locality in Coulomb scattering has always been controversial, and more precise measurements or multi-particle simulations would be desirable to help finally settle this old issue.

It is well-known from Piwinski that in the smooth approximation, in the uncoupled case, an equilibrium exists below transition and not above transition. When a real varying lattice is used, no equilibrium exists, even below transition. We have given a simple argument to understand this for a special case; however, a result of B-M for the total phase space growth rate shows that the lack of equilibrium in a non-smooth machine is a general result. In the original paper, this fact was pointed out for the case of a varying dispersion function, but we find that this understates the fact. Even disregarding dispersion and considering varying $\beta_{x, y}$ leads to this conclusion.

Finally, as mentioned, for the case of the smooth approximation, the information 
about equilibrium can be understood via Piwinski's invariant. We have generalized this invariant for the case of a storage ring near a synchrobetatron sum or difference resonance. We find that with strong enough coupling, an equilibrium doesn't exist below transition, even in the smooth approximation. 


\section{Appendix A}

\section{Further Invariant Calculations}

We would like to show that the expressions given for the invariants $G_{a}$ in the text, do in fact satisfy the invariant condition. That is, we would like to show that

$$
G_{a}=J U H_{a} U^{T} J
$$

satisfies

$$
M^{T} G_{a} M=G_{a}
$$

Using the symplectic property of $M$, and Eq. (2.17), we derive

$$
\begin{aligned}
M^{T} G_{a} M & =M^{T} J U H_{a} U^{T} J M \\
& =J M^{-1} U H_{a} U^{T} M^{T-1} J \\
& =J U e^{-\Lambda} H_{a} e^{-\Lambda} U^{T} J \\
& =J U H_{a} U^{T} J
\end{aligned}
$$

In the last step we have done the matrix multiplication

$$
\left(\begin{array}{cc}
e^{-i \mu} & 0 \\
0 & e^{i \mu}
\end{array}\right)\left(\begin{array}{ll}
0 & 1 \\
1 & 0
\end{array}\right)\left(\begin{array}{cc}
e^{-i \mu} & 0 \\
0 & e^{i \mu}
\end{array}\right)=\left(\begin{array}{ll}
0 & 1 \\
1 & 0
\end{array}\right)
$$

This shows that the $G_{a}$ given are in fact invariants.

Now we consider the beam distribution written in terms of the invariants. We 
write a general (6-D) Gaussian distribution as

$$
f(\vec{z})=\frac{1}{(2 \pi)^{3} \sqrt{\operatorname{det}\left(\mathbb{M}^{-1}\right)}} e^{-\frac{1}{2} \vec{z}^{T} \mathbb{M} \vec{z}}
$$

where we have normalized $f$ so that $\int f(\vec{z}) d \vec{z}=1$. By performing the integration, one can show that the second moment matrix is given by

$$
\Sigma_{j k}=\left\langle z_{j} z_{k}\right\rangle=\mathbb{M}_{j k}^{-1}
$$

In terms of the three invariants, we can write the distribution as

$$
f(\vec{z})=\frac{1}{\pi^{3}\left\langle g_{1}\right\rangle\left\langle g_{2}\right\rangle\left\langle g_{3}\right\rangle} \exp \left(-\frac{g_{1}}{\left\langle g_{1}\right\rangle}-\frac{g_{2}}{\left\langle g_{2}\right\rangle}-\frac{g_{3}}{\left\langle g_{3}\right\rangle}\right)
$$

Let us prove this. In particular, we must show that the quantities we have written as $\left\langle g_{a}\right\rangle$ in the above equation are indeed given by

$$
\left\langle g_{a}\right\rangle=\int d \vec{z} g_{a} f(\vec{z})
$$

To show this, we introduce the matrix $K$ given by

$$
K=\frac{1}{\sqrt{2}}\left(\begin{array}{cccccc}
1 & -i & 0 & 0 & 0 & 0 \\
-i & 1 & 0 & 0 & 0 & 0 \\
0 & 0 & 1 & -i & 0 & 0 \\
0 & 0 & -i & 1 & 0 & 0 \\
0 & 0 & 0 & 0 & 1 & -i \\
0 & 0 & 0 & 0 & -i & 1
\end{array}\right)
$$

Note that $\operatorname{det}(K)=1$. In fact, it is symplectic. Now, consider the change of coordinates from $\vec{z}$ to the coordinates $\vec{V}$

$$
\vec{z}=U K \vec{V}
$$

$U K$ is in fact a real symplectic matrix. Its columns are $\sqrt{2}$ times the real and 
imaginary parts of $v_{a}$. The quadratic form $g_{a}$ becomes

$$
\begin{aligned}
g_{a} & =\vec{z}^{T} G_{a} \vec{z} \\
& =\vec{V}^{T} K^{T} U^{T} G_{a} U K \vec{V} \\
& =\vec{V}^{T} K^{T} H_{a} K \vec{V} \\
& =\vec{V}^{T} I_{a} \vec{V}
\end{aligned}
$$

Where $I_{a}$ is a matrix that is all zero's except for a $2 \times 2$ identity matrix in the ath diagonal $2 \times 2$ block. Thus, $g_{a}=V_{a}^{2}+V_{-a}^{2}$ (with $a=1,2,3$ for $g_{a}$, and $k= \pm 1, \pm 2, \pm 3$ for $V_{k}$ ). Applying this change of variables to (A.8), we get

$$
\begin{gathered}
\int d \vec{V} \frac{V_{a}^{2}+V_{-a}^{2}}{\pi^{3}\left\langle g_{1}\right\rangle\left\langle g_{2}\right\rangle\left\langle g_{3}\right\rangle} e^{-\frac{V_{1}^{2}+V_{-1}^{2}}{\left\langle g_{1}\right\rangle}-\frac{V_{2}^{2}+V_{-2}^{2}}{\left\langle g_{2}\right\rangle}-\frac{V_{3}^{2}+V_{-3}^{2}}{\left\langle g_{3}\right\rangle}} \\
=\left\langle g_{a}\right\rangle
\end{gathered}
$$

after doing the 6 1-D integrals.

Next we find expressions for the second moment matrix. Looking at (A.7),(A.5) and (2.19), we identify

$$
\mathbb{M}=\frac{2 G_{1}}{\left\langle g_{1}\right\rangle}+\frac{2 G_{2}}{\left\langle g_{2}\right\rangle}+\frac{2 G_{3}}{\left\langle g_{3}\right\rangle}
$$

Thus, to find the second moment matrix $\Sigma$, we must invert this matrix. We claim that the inverse is given by

$$
\mathbb{M}^{-1}=-\frac{1}{2}\left\langle g_{1}\right\rangle J G_{1} J-\frac{1}{2}\left\langle g_{2}\right\rangle J G_{2} J-\frac{1}{2}\left\langle g_{3}\right\rangle J G_{3} J
$$

In order to prove this, we show the following:

$$
J G_{a} J G_{b}=-\delta_{a b} I_{a}
$$

where $a$ and $b$ run from 1 to 3 . Now, $G_{a}$ is written explicitly as

$$
G_{a}=J U H_{a} U^{T} J
$$

Substituting this in and using the symplectic property of $U$ and the fact that $J^{2}=-I$, 
we get

$$
J G_{a} J G_{b}=U H_{a} J H_{b} J U^{-1}
$$

From this and the definition of $H_{a},(2.20)$, Eq. (A.15) follows by simple matrix multiplication. In particular, when $a=b$, we find

$$
\sigma_{x} J_{2} \sigma_{x} J_{2}=\left(\begin{array}{ll}
1 & 0 \\
0 & 1
\end{array}\right)
$$

The minus sign comes from the two factors of $i$ from the $H_{a}$. Now, multiplication of (A.13) by (A.14) and repeated use of Eq. (A.15), proves that (A.14) is indeed the inverse matrix. 


\section{Appendix B}

\section{General Perturbation Theory}

Let us suppose we have a matrix $M$ that can be expanded as

$$
M=\sum_{p} M_{p}
$$

where we consider $M_{p}$ to be of order $\epsilon^{p}$ where $\epsilon$ is some small parameter. We seek a perturbation expansion of the eigenvectors and eigenvalues of $M$ :

$$
M v_{k}=\lambda_{k} v_{k}
$$

with

$$
v_{k}=\sum_{p} \tilde{v}_{k p}
$$

and

$$
\lambda_{k}=\sum_{p} \lambda_{k p}
$$

We consider $\tilde{v}_{k p}$ and $\lambda_{k p}$ to be of order $\epsilon^{p}$. We use the tilde in $\tilde{v}_{k p}$ because we would like to distinguish $\tilde{v}_{k 0}$ from $v_{k 0}$. Let $Z_{d g}(k)$ be the indices such that for $j \in Z_{d g}(k)$, $\lambda_{j 0}=\lambda_{k 0}$. In other words, $Z_{d g}(k)$ is the set of indices with eigenvalues that are degenerate with $\lambda_{k 0}$. We let $\left\{v_{k 0}\right\}$ be an orthonormal complete set of eigenvectors of 
$M_{0}$. In particular, we assume that we can define covectors $v^{l 0}$ such that

$$
v^{l 0} v_{k 0}=\delta_{l k}
$$

How to do this for a symplectic matrix is discussed in the main text. This means that we can expand an arbitrary vector as

$$
v=\sum_{k} c_{k} v_{k 0}
$$

with

$$
c_{k}=v^{k 0} v
$$

We consider the $v_{k 0}$ to be the "uncoupled", "unperturbed" eigenvectors. Within the perturbation matrices $M_{1}$ and $M_{2}$, we consider there as being two different types of perturbations. In the case that we consider them both as 1st order (an important case will occur when we consider it as 2nd order), we notate them as $M_{1 \xi}$ and $M_{1 \delta \mu}$. When just $M_{1 \delta \mu}$ is added to $M_{0}$, its effect is to change the eigenvalues, but not the $v_{k 0}$. This is in effect how we define $v_{k 0}$. The addition of the perturbation $M_{1 \xi}$ serves to pick out a particular linear combination of the $v_{k 0}$ which we notate as $\tilde{v}_{k 0}$, in addition to causing a perturbation of these eigenvectors. However, it will be the main purpose of this section to derive the $\tilde{v}_{k 0}$ for the two cases where we have a $M_{1 \delta \mu}$ and a $M_{2 \delta \mu}$, i.e. when the purely eigenvalue changing perturbation is first order and second order. It will turn out that this latter case is important in the case of "coupling" causing an integer or half integer resonance.

We now consider the eigenvalue equation setting equal terms of the same order up to 2nd order:

$$
\begin{aligned}
p & =0: & M_{0} \tilde{v}_{k 0} & =\lambda_{k 0} \tilde{v}_{k 0} \\
p & =1: & M_{0} \tilde{v}_{k 1}+M_{1} \tilde{v}_{k 0} & =\lambda_{k 0} \tilde{v}_{k 1}+\lambda_{k 1} \tilde{v}_{k 0} \\
p & =2: & M_{0} \tilde{v}_{k 2}+M_{1} \tilde{v}_{k 1}+M_{2} \tilde{v}_{k 0} & =\lambda_{k 0} \tilde{v}_{k 2}+\lambda_{k 1} \tilde{v}_{k 1}+\lambda_{k 0} \tilde{v}_{k 2}
\end{aligned}
$$

Consider the $p=0$ equation. Expanding out the $\tilde{v}_{k 0}$ and using the 0th order 
eigenvector equation, we get

$$
\sum_{j} c_{k 0}^{j} \lambda_{j 0} v_{j 0}=\lambda_{k 0} \sum_{j} c_{k 0}^{j} v_{j 0}
$$

Multiplying on the left by $v^{l 0}$ gives

$$
c_{k 0}^{l} \lambda_{l 0}=c_{k 0}^{l} \lambda_{k 0}
$$

which implies that either $\lambda_{l 0}=\lambda_{k 0}$, or $c_{k 0}^{l}=0$. This is what we expect since for $l \in Z_{d g}(k), \lambda_{l 0}=\lambda_{k 0}$ and otherwise $c_{k 0}^{l}=0$.

Next we consider $p=1$. Expanding the $\tilde{v}_{k}$ and again using the 0th order eigenvector equation gives

$$
\begin{aligned}
& \sum_{j} c_{k 1}^{j} \lambda_{j 0} v_{j 0}+M_{1} \sum_{j} c_{k 0}^{j} v_{j 0} \\
= & \lambda_{k 0} \sum_{j} c_{k 1}^{j} v_{j 0}+\lambda_{k 1} \sum_{j} c_{k 0}^{j} v_{j 0}
\end{aligned}
$$

We now multiply on the left by $v^{l 0}$. The result is

$$
c_{k 1}^{l} \lambda_{l 0}+\sum_{j} c_{k 0}^{j} \mathcal{M}_{l j}=\lambda_{k 0} c_{k 1}^{l}+\lambda_{k 1} c_{k 0}^{l}
$$

where we have defined

$$
\mathcal{M}_{l j}=v^{l 0} M_{1} v_{j 0}
$$

Now, suppose that $l \in Z_{d g}(k)$. Then $\lambda_{l 0}=\lambda_{k 0}$ and two of the terms cancel. We are left with

$$
\sum_{j} c_{k 0}^{j} \mathcal{M}_{l j}=\lambda_{k 1} c_{k 0}^{l} \quad l \in Z_{d g}(k)
$$

We will use this equation to analyze the sum and difference resonances. In the case where there is no degeneracy, $Z_{d g}(k)=\{k\}$ and we get the usual result from 1st order 
non-degenerate perturbation theory:

$$
\lambda_{k 1}=\mathcal{M}_{k k}
$$

Next suppose that $l \notin Z_{d g}(k)$. Then $\lambda_{l 0} \neq \lambda_{k 0}$ and $c_{k 0}^{l}=0$. Then Eq. (B.12) gives

$$
c_{k 1}^{l}=\frac{\sum_{j} c_{k 0}^{j} \mathcal{M}_{l j}}{\lambda_{k 0}-\lambda_{l 0}} \quad l \notin Z_{d g}(k)
$$

Finally, we consider $p=2$. Expanding out the $\tilde{v}_{k p}$, using the $M_{0}$ eigenvalue equation and left multiplying by $v^{l 0}$ gives

$$
\begin{array}{r}
c_{k 2}^{l} \lambda_{l 0}+\sum_{j} c_{k 1}^{j} \mathcal{M}_{l j}+\sum_{j} c_{k 0}^{j} \mathcal{M}_{2, l j} \\
=\lambda_{k 0} c_{k 2}^{l}+\lambda_{k 1} c_{k 1}^{l}+\lambda_{k 2} c_{k 0}^{l}
\end{array}
$$

where we have defined

$$
\mathcal{M}_{2, l j} \equiv v^{l 0} M_{2} v_{j 0}
$$

Now, suppose that $l \in Z_{d g}(k)$. Then $\lambda_{l 0}=\lambda_{k 0}$. We can then write

$$
c_{k 0}^{l} \lambda_{k 2}=\sum_{j} c_{k 1}^{j} \mathcal{M}_{l j}+\sum_{j} c_{k 0}^{j} \mathcal{M}_{2, l j}-\lambda_{k 1} c_{k 1}^{l} \quad l \in Z_{d g}(k)
$$

There are two different contexts in which one might use this equation. The first is that the $c_{k 0}^{l}$ have already been determined by the lower order equations and this becomes an equation for just $\lambda_{k 2}$. This is the case if $M_{1}$ breaks the degeneracy. In this case $\lambda_{k 1}$ is nonzero. We then think of this equation as adding small terms to the eigenvectors and eigenvalues. Another important possibility is where $M_{1}$ does not break the degeneracy. This happens when all the $\mathcal{M}_{l k}$ 's are zero for $l \in Z_{d g}(k)$. This means that $\lambda_{k 1}=0$. Then, after using Eq. (B.12), (B.19) becomes an eigenvalue equation for $c_{k 0}^{l}$ and $\lambda_{k 2}$ :

$$
\sum_{j}\left[\sum_{n \notin Z_{d g}(k)} \frac{\mathcal{M}_{n j} \mathcal{M}_{l n}}{\lambda_{k 0}-\lambda_{n 0}}+\mathcal{M}_{2, l j}\right] c_{k 0}^{j}=\lambda_{k 2} c_{k 0}^{l}
$$




$$
l \in Z_{d g}(k)
$$

This is our main result which we use to analyze the integer and half-integer resonances caused by coupling.

If we take the case where there is no degeneracy, this reduces to

$$
\lambda_{k 2}=\sum_{n \neq k} \frac{\mathcal{M}_{n k} \mathcal{M}_{k n}}{\lambda_{k 0}-\lambda_{n 0}}+\mathcal{M}_{2, k k}
$$

which, if we also set $\mathcal{M}_{2, k k}=0$ gives the usual expression for the second order shift in the eigenvalue for non-degenerate perturbation theory.

Now, let $l \notin Z_{d g}(k)$. Then, as before, $\lambda_{l 0} \neq \lambda_{k 0}$ and $c_{k 0}^{l}=0$. Solving for $c_{k 2}^{l}$ gives

$$
c_{k 2}^{l}=\frac{\sum_{j} c_{k 1}^{j} \mathcal{M}_{l j}+\sum_{j} c_{k 0}^{j} \mathcal{M}_{2, l j}-\lambda_{k 1} c_{k 1}^{l}}{\lambda_{k 0}-\lambda_{l 0}}
$$




\section{Appendix C}

\section{Relationship between $\xi_{+}$and $\xi_{-}$}

Suppose we have a general matrix $Q$ with elements

$$
Q=\left(\begin{array}{ll}
q_{11} & q_{12} \\
q_{21} & q_{22}
\end{array}\right)
$$

Now, compute the quantities

$$
\begin{aligned}
\frac{1}{2}\left(\begin{array}{ll}
i & 1
\end{array}\right) Q\left(\begin{array}{l}
1 \\
i
\end{array}\right) & =\frac{1}{2}\left[\left(q_{21}-q_{12}\right)+i\left(q_{11}+q_{22}\right)\right] \\
\frac{1}{2}\left(\begin{array}{ll}
-i & 1
\end{array}\right) Q\left(\begin{array}{l}
1 \\
i
\end{array}\right) & =\frac{1}{2}\left[\left(q_{21}+q_{12}\right)+i\left(-q_{11}+q_{22}\right)\right]
\end{aligned}
$$

Now, defining

and identifying

$$
\mathcal{A}_{x, z}=\left(\begin{array}{cc}
\sqrt{\beta_{x, z}} & 0 \\
\frac{-\alpha_{x, z}}{\sqrt{\beta_{x, z}}} & \frac{1}{\sqrt{\beta_{x, z}}}
\end{array}\right)
$$

$$
Q=\mathcal{A}_{x}^{T} J B \mathcal{A}_{z}
$$

where $B$ is the upper-right $2 \times 2$ block of $M_{1}$ or $P$, we can see that

$$
\left|\mathcal{M}_{12}\right|^{2}-\left|\mathcal{M}_{-12}\right|^{2}=\operatorname{det}(Q)
$$


But, since $\operatorname{det}\left(\mathcal{A}_{x, z}\right)=\operatorname{det}(J)=1$, we get that $\operatorname{det}(Q)=\operatorname{det}(B)$, and hence

$$
\xi_{-}^{2}-\xi_{+}^{2}=4 \operatorname{det}(B)
$$




\section{Appendix D}

\section{Coupling Integer/Half Integer Resonances}

Suppose that the transfer matrix $M$ is close to the $\mu_{z}$ integer resonance. Then the eigenvalue equation can be written in the form ${ }^{1}$

$$
\left(\begin{array}{cc}
M_{x} & B \\
C & I+D
\end{array}\right)\left(\begin{array}{l}
u \\
v
\end{array}\right)=(1+\delta \lambda)\left(\begin{array}{l}
u \\
v
\end{array}\right)
$$

where we are looking for the eigenvalues near 1 . $u$ and $v$ are $1 \times 2$ column matrices, and we assume that $B, C, D$ and $\delta \lambda$ are small. If the upper equation is solved for $u$, we find

$$
u=-\left[M_{x}-I(1+\delta \lambda)\right]^{-1} v
$$

Substituting this into the lower equation and taking lowest order in the small quantities (i.e. ignoring $\delta \lambda$ in above equation), we get

$$
\left[D-C\left(M_{x}-I\right)^{-1} B\right] v=\delta \lambda v
$$

Thus, we must find eigenvalues and eigenvectors of this $2 \times 2$ matrix. If we change into the basis in which $M$ is diagonal, and taking into account the half-integer resonance

\footnotetext{
${ }^{1}$ Thanks to B. Freivogel for pointing out this alternative derivation of the 2 nd order degenerate perturbation theory results.
} 
as well, in which $I \rightarrow-I$, we get

$$
\left(\begin{array}{cc}
\frac{\mathcal{M}_{12} \mathcal{M}_{21}}{\lambda_{20}-\lambda_{10}} & \frac{\mathcal{M}_{1-2} \mathcal{M}_{21}}{\lambda_{20}-\lambda_{10}} \\
+\frac{\mathcal{M}_{-12} \mathcal{M}_{2-1}}{\lambda_{20}-\lambda_{-10}} & +\frac{\mathcal{M}_{-1-2} \mathcal{M}_{2-1}}{\lambda_{20}-\lambda_{-10}} \\
+\mathcal{M}_{22} & +\mathcal{M}_{2-2} \\
& \\
\frac{\mathcal{M}_{12} \mathcal{M}_{-21}}{\lambda_{20}-\lambda_{10}} & \frac{\mathcal{M}_{1-2} \mathcal{M}_{-21}}{\lambda_{20}-\lambda_{10}} \\
+\frac{\mathcal{M}_{-12} \mathcal{M}_{-2-1}}{\lambda_{20}-\lambda_{-10}} & +\frac{\mathcal{M}_{-1-2} \mathcal{M}_{-2-1}}{\lambda_{20}-\lambda_{-10}} \\
+\mathcal{M}_{-22} & +\mathcal{M}_{-2-2}
\end{array}\right)
$$

For the integer resonance, this simplifies to

$$
\left(\begin{array}{cc}
\frac{\left|r_{12}\right|^{2}}{1-e^{-i \mu_{x}}}-\frac{\left|r_{-12}\right|^{2}}{1-e^{i \mu_{x}}}+r_{22} & i r_{21} r_{1-2} \cot \left(\frac{\mu_{x}}{2}\right)+r_{2-2} \\
-i r_{21}^{*} r_{1-2}^{*} \cot \left(\frac{\mu_{x}}{2}\right)+r_{-22} & \frac{\left|r_{12}\right|^{2}}{1-e^{i \mu_{x}}}-\frac{\left|r_{-12}\right|^{2}}{1-e^{-i \mu_{x}}}+r_{-2-2}
\end{array}\right)
$$

for the half integer it is

$$
\left(\begin{array}{cc}
\frac{-\left|r_{12}\right|^{2}}{1+e^{-i \mu_{x}}}+\frac{\left|r_{-12}\right|^{2}}{1+e^{\mu_{x}}}-r_{22} & i r_{21} r_{1-2} \tan \left(\frac{\mu_{x}}{2}\right)-r_{2-2} \\
-i r_{21}^{*} r_{1-2}^{*} \tan \left(\frac{\mu_{x}}{2}\right)-r_{-22} & \frac{-\left|r_{12}\right|^{2}}{1+e^{i \mu_{x}}}+\frac{\left|r_{-12}\right|^{2}}{1+e^{-i \mu_{x}}}-r_{-2-2}
\end{array}\right)
$$

Now, for a matrix,

$$
\left(\begin{array}{ll}
a & b \\
c & d
\end{array}\right)
$$

the eigenvalues can be written as

$$
\delta \lambda_{ \pm}=\frac{1}{2}\left[a+d \pm(a-d) \sqrt{1+\frac{4 b c}{(a-d)^{2}}}\right]
$$

and the (unnormalized) eigenvectors as

$$
v_{ \pm}=\left(\begin{array}{c}
\frac{1}{2 c}\left[(a-d) \pm(a-d) \sqrt{1+\frac{4 b c}{(a-d)^{2}}}\right] \\
1
\end{array}\right)
$$

In order to be consistent with the notation for the simpler resonances, we make the following definitions

$$
\Delta \mu=-i e^{-i \mu_{0}}(a-d)
$$




$$
\xi=2|b|
$$

and

$$
\phi=\arg \left(e^{i \mu_{0}} b\right)
$$

where $\mu_{0}$ is 0 for the integer resonance and $\pi$ for the half integer. This phase definition being consistent depends on the fact that $c^{*}=b$ which is easily verified for each of the two cases above. The perturbed phase advance is given by

$$
\mu_{2}=\Delta \mu_{n z}^{2} \sqrt{1-\frac{\xi_{n z}^{2}}{\Delta \mu_{n z}^{2}}}
$$

for the integer resonance and

$$
\mu_{2}=\pi+\Delta \mu_{\frac{n}{2} z}^{2} \sqrt{1-\frac{\xi_{\frac{n}{2} z}^{2}}{\Delta \mu_{\frac{n}{2} z}^{2}}}
$$

for the half integer resonance. The perturbed eigenvector is given by

$$
v_{2}=\cosh \left(\frac{\theta}{2}\right) v_{z}-i e^{-i \phi} \sinh \left(\frac{\theta}{2}\right) v_{-z}
$$

with

$$
\tanh (\theta)=\frac{\xi}{\Delta \mu}
$$

Writing out all of these quantities explicitly, and after some algebra, we find for the integer resonance

$$
\begin{gathered}
\Delta \mu_{n z}=2 \mu_{z}-2 i r_{22 \xi}-\left(\left|r_{12}\right|^{2}+\left|r_{-12}\right|^{2}\right) \cot \left(\frac{\mu_{x}}{2}\right) \\
\xi_{n z}=2\left|r_{2-2}+i r_{1-2} r_{21} \cot \left(\frac{\mu_{x}}{2}\right)\right|
\end{gathered}
$$

and

$$
\phi_{n z}=\arg \left[r_{2-2}+i r_{1-2} r_{21} \cot \left(\frac{\mu_{x}}{2}\right)\right]
$$


For the half integer we get

$$
\begin{gathered}
\Delta \mu_{\frac{n}{2} z}=2\left(\mu_{z}-\pi\right)-2 i r_{22 \xi}+\left(\left|r_{12}\right|^{2}+\left|r_{-12}\right|^{2}\right) \tan \left(\frac{\mu_{x}}{2}\right) \\
\xi_{\frac{n}{2} z}=2\left|r_{2-2}-i r_{1-2} r_{21} \tan \left(\frac{\mu_{x}}{2}\right)\right|
\end{gathered}
$$

and

$$
\phi_{\frac{n}{2} z}=\arg \left[r_{2-2}-i r_{1-2} r_{21} \tan \left(\frac{\mu_{x}}{2}\right)\right]
$$

In each case, we can combine $\xi$ and $\Delta \mu$ to find the perturbed value of $\mu_{2}$ using (D.13) and (D.14). We ignore the issue of the sign here and compute $\mu_{2}^{2}$ :

$$
\begin{aligned}
\mu_{2}^{2}= & \left(\mu_{z}-i r_{22 \xi}\right)^{2}-\left(\mu_{z}-i r_{22 \xi}\right)\left(\left|r_{12}\right|^{2}+\left|r_{1-2}\right|^{2}\right) \cot \left(\frac{\mu_{x}}{2}\right)-\left|r_{2-2 \xi}\right|^{2} \\
& +\frac{1}{4}\left(\left|r_{12}\right|^{2}-\left|r_{1-2}\right|^{2}\right) \cot ^{2}\left(\frac{\mu_{x}}{2}\right)+\frac{1}{2} \operatorname{Im}\left(r_{2-2 \xi}^{*} r_{12} r_{1-2}\right) \cot \left(\frac{\mu_{x}}{2}\right) \\
\left(\mu_{2}-\pi\right)^{2}= & {\left[\left(\mu_{z}-\pi\right)-i r_{22 \xi}\right]^{2}+\left(\left(\mu_{z}-\pi\right)-i r_{22 \xi}\right)\left(\left|r_{12}\right|^{2}+\left|r_{1-2}\right|^{2}\right) \tan \left(\frac{\mu_{x}}{2}\right)-\left|r_{2-2 \xi}\right|^{2} } \\
& +\frac{1}{4}\left(\left|r_{12}\right|^{2}-\left|r_{1-2}\right|^{2}\right) \tan ^{2}\left(\frac{\mu_{x}}{2}\right)-\frac{1}{2} \operatorname{Im}\left(r_{2-2 \xi}^{*} r_{12} r_{1-2}\right) \tan \left(\frac{\mu_{x}}{2}\right)
\end{aligned}
$$

for the integer and half-integer $z$ resonances respectively. Note that for both cases $\mu_{2}$ reduces to $\mu_{z}$ when the perturbation is turned off.

Now, we consider a useful special case. Suppose that the perturbation $M_{1 \xi}$ only has off-block diagonal elements. This implies that $r_{22 \xi}=r_{2-2 \xi}=0$. Further suppose that $\left|r_{12}\right|^{2}=\left|r_{-12}\right|^{2}$. This is equivalent to $\operatorname{det}(B)=0$, where $B$ is the off-block diagonal matrix in either $M_{1 \xi}$ or $P$. If these two conditions hold, the expressions for $\mu_{2}$ simplify considerably. They become

$$
\begin{aligned}
\mu_{2}^{2} & =\mu_{z}^{2}-2 \mu_{z}\left|r_{12}\right|^{2} \cot \left(\frac{\mu_{x}}{2}\right) \\
\left(\mu_{2}-\pi\right)^{2} & =\left(\mu_{z}-\pi\right)^{2}+2\left(\mu_{z}-\pi\right)\left|r_{12}\right|^{2} \tan \left(\frac{\mu_{x}}{2}\right)
\end{aligned}
$$

for the integer and half integer respectively.

The expressions for $x$ are given by interchanging 1 and 2 , and $x$ and $z$. 
Finally, consider the quantity $\bar{\mu}$ which we expect to be 0 for the integer and halfinteger resonances. It is proportional to the trace of (D.4). Computing this quantity, we find that it is not zero. A careful analysis, however, reveals that it is always higher order than the lowest order terms kept and can thus be seen as a part of the higher order terms in the expansion of $e^{i \mu}$ which should be dropped. 


\section{Appendix E}

\section{IBS Calculations}

Consider two particles scattering with phase space positions $\vec{Z}_{1}=\left(\vec{X}_{1}, \vec{P}_{1}\right)$ and $\vec{Z}_{2}=$ $\left(\vec{X}_{2}, \vec{P}_{2}\right)$. Define $\vec{r}=\vec{X}_{1}-\vec{X}_{2}$ and $\vec{\Delta}=\vec{P}_{1}-\vec{P}_{2}$. Now if the two particles were to continue in the straight lines determined by their initial conditions, their motion would be given by

$$
\vec{X}_{1}(t)=\vec{X}_{1}+\frac{\vec{P}_{1}}{m} t, \quad \vec{X}_{2}(t)=\vec{X}_{2}+\frac{\vec{P}_{2}}{m} t
$$

and their distance apart at time $t$ would be

$$
\vec{r}(t)=\vec{r}+\frac{\vec{\Delta}}{m} t \quad \text { or } \quad r^{2}(t)=r^{2}+\frac{2 \vec{r} \cdot \vec{\Delta}}{m} t+\frac{\Delta^{2}}{m^{2}} t^{2} .
$$

They would reach a minimum distance apart at a time (when $r^{2}(t)$ is minimized)

$$
t_{\min }=-\frac{m r}{\Delta} \hat{\Delta} \cdot \hat{r}
$$

The vector separating the two particles at $t_{\min }$ can be thought of as the impact parameter $\vec{b}$ for the scattering of the two particles. It is given by

$$
\vec{b} \equiv \vec{r}\left(t_{\min }\right)=\vec{r}-r(\hat{\Delta} \cdot \hat{r}) \hat{\Delta}
$$


Thus, we have

$$
b^{2}=r^{2}\left(1-(\hat{\Delta} \cdot \hat{r})^{2}\right)=r^{2}\left(1-\cos ^{2} \chi\right)
$$

with

$$
\hat{\Delta} \cdot \hat{r}=\cos \chi
$$

so that

$$
b=r \sin \chi
$$

If we assume the initial conditions can be thought of as infinitely far apart as far as the scattering goes, we can relate the scattering angle $\psi$ to the impact parameter $b$. The scattering will occur in the $\hat{b}-\hat{\Delta}$ plane. By conservation of momentum, we have that

$$
\delta \vec{P}_{1}=-\delta \vec{P}_{2}
$$

so that

$$
\delta \vec{P}_{1}=\frac{1}{2}\left(\left(\vec{P}_{1}^{\prime}-\vec{P}_{1}\right)-\left(\vec{P}_{2}^{\prime}-\vec{P}_{2}\right)\right)=\frac{1}{2}\left(\left(\vec{P}_{1}^{\prime}-\vec{P}_{2}^{\prime}\right)-\left(\vec{P}_{1}-\vec{P}_{2}\right)\right)=\frac{1}{2} \delta \vec{\Delta}
$$

These results imply that

$$
\delta \vec{P}_{1}=\frac{1}{2} \Delta((\cos \psi-1) \hat{\Delta}+\sin \psi \hat{b})
$$

and given the coulomb interaction one can show that

$$
\tan \left(\frac{\psi}{2}\right)=\frac{2(m c)^{2} r_{0}}{\Delta^{2} b}=\frac{2 k}{\Delta^{2} b} \quad k=(m c)^{2} r_{0}
$$

where $r_{0}$ is the classical particle radius. If we assume that small angles dominate, it will be usefull to express $\delta \vec{P}_{1}$ in this limit:

$$
\delta \vec{P}_{1} \approx-\frac{4 k^{2}}{\Delta^{3} b^{2}} \hat{\Delta}+\frac{2 k}{\Delta b} \hat{b} \quad \psi<<1
$$

This equation says that the particle will receive a kick in opposite direction to its relative momentum with another particle and in the same direction as its vector 
impact parameter with that particle. The first of these terms causes a damping effect tending to slow particles down in their direction of travel and is termed "dynamical friction". The second term causes both the space charge and diffusive effects.

To calculate the diffusion and drift coefficients for a particle in this distribution, we compute the quantities $\left\langle\delta P_{1 i}\right\rangle$ and $\left\langle\delta P_{1 i} \delta P_{1 j}\right\rangle$ (we leave off the 1 after this) in a time $\Delta t$ resulting from all the other particles in the distribution. Now, there is a sense in which any given particle is undergoing the scattering process with all other particles at all times. However, the majority of the effect from a given scatter occurs within the short period of time in which the particles obtain their distance of closest approach. Thus, in order to use the scattering approach, we will let the entire scattering effect occur at one time, namely the time of closest approach.

\section{Reduction to Rosenbluth Potentials}

Let us first suppose that

$$
f(\vec{x}, \vec{p})=\rho_{x}(\vec{x}) \rho_{p}(\vec{p})
$$

then, doing the integral over $\cos \chi=\hat{\Delta} \cdot \hat{r}$ using the $\delta$ function for fixed $\hat{\Delta}$ we get

$$
\frac{<\delta p_{i}>}{\Delta t} \approx-\frac{k^{2}}{4 m} \int d \vec{\Delta} \rho_{p}\left(\vec{p}_{2}\right) d^{2} \vec{b} \frac{\hat{\Delta}_{i}}{\Delta^{2} b^{2}} g(\vec{b} ; \hat{\Delta})
$$

and

$$
\frac{<\delta p_{i} \delta p_{j}>}{\Delta t} \approx \frac{k^{2}}{4 m} \int d \vec{\Delta} d^{2} \vec{b} \frac{\hat{b}_{i} \hat{b}_{j}}{\Delta b^{2}} g\left(\vec{b} ; \vec{x}_{1}, \hat{\Delta}\right)
$$

where we've defined

$$
g\left(\vec{b} ; \vec{x}_{1}, \hat{\Delta}\right)=\rho_{x}\left(\vec{x}_{1}-\vec{b}\right)
$$

Note that we canceled one of the $b$ 's in the denominator in going from $\int d^{3} \vec{r}$ to $\int d^{2} \vec{b}$, since $b=r$ when $\cos \chi=0$. Let's look at the $\vec{b}$ integration. For $\delta p_{i}$, we have

$$
I_{i}=\hat{\Delta}_{i} \int d b d \alpha \frac{g(\vec{b})}{b}
$$


and for $\delta p_{i} \delta p_{j}$, we have

$$
I_{i j}=\int d b d \alpha \frac{g(\vec{b}) \hat{b}_{i} \hat{b}_{j}}{b}
$$

Now, suppose we set $g(\vec{b})$ equal to a constant- $f\left(\vec{x}_{1}, \vec{p}_{1}\right)$. Then the integrals diverge logarithmically. Let's cut them off at a maximum $b_{\max }$ and minimum $b_{\min }$. The integral for $\delta p_{i}$ becomes simply

$$
I_{i}=2 \pi f\left(\vec{x}_{1}, \vec{p}_{1}\right) \log \frac{b_{\max }}{b_{\min }} \hat{\Delta}_{i}
$$

For $\delta p_{i} \delta p_{j}$ we get

$$
I_{i j}=f\left(\vec{x}_{1}, \vec{p}_{1}\right) \log \frac{b_{\max }}{b_{\min }} \int_{0}^{2 \pi} \hat{b}_{i} \hat{b}_{j}
$$

Using $\hat{b}=\cos \alpha \hat{b}_{1}+\sin \alpha \hat{b}_{2}$ we get

$$
\int_{0}^{2 \pi} \hat{b}_{i} \hat{b}_{j} d \alpha=\frac{1}{2}\left(\hat{b}_{1 i} \hat{b}_{1 j}+\hat{b}_{2 i} \hat{b}_{2 j}\right)
$$

Now, consider the quantity

$$
\begin{gathered}
\hat{b}_{1 i} \hat{b}_{1 j}+\hat{b}_{2 i} \hat{b}_{2 j}+\hat{\Delta}_{i} \hat{\Delta}_{j} \\
=\left(\hat{b}_{1} \cdot \hat{x}_{i}\right)\left(\hat{b}_{1} \cdot \hat{x}_{j}\right)+\left(\hat{b}_{2} \cdot \hat{x}_{i}\right)\left(\hat{b}_{2} \cdot \hat{x}_{j}\right)+\left(\hat{\Delta} \cdot \hat{x}_{i}\right)\left(\hat{\Delta} \cdot \hat{x}_{j}\right) \\
=\hat{x}_{i} \cdot \hat{x}_{j} \\
=\delta_{i j}
\end{gathered}
$$

Thus,

$$
\hat{b}_{1 i} \hat{b}_{1 j}+\hat{b}_{2 i} \hat{b}_{2 j}=\delta_{i j}-\hat{\Delta}_{i} \hat{\Delta}_{j}
$$

So

$$
I_{i j}=\frac{1}{2} f\left(\vec{x}_{1}, \vec{p}_{1}\right) \log \frac{b_{\max }}{b_{\min }}\left(\delta_{i j}-\hat{\Delta}_{i} \hat{\Delta}_{j}\right)
$$

So we have

$$
\frac{<\delta p_{i}>}{\Delta t} \approx-\frac{k^{2}}{4 m} \int d \vec{\Delta} \rho_{p}\left(\vec{p}_{2}\right) \frac{I_{i}}{\Delta^{2}}
$$


and

$$
\frac{<\delta p_{i} \delta p_{j}>}{\Delta t} \approx \frac{k^{2}}{4 m} \int d \vec{\Delta} \rho_{p}\left(\vec{p}_{2}\right) \frac{I_{i j}}{\Delta}
$$

These correspond to the Rosenbluth potentials[10].

Even though the damping and diffusion coefficients due to IBS are dependent on the phase space position, one may wonder whether one can find some appropriate constant values, such as taking the values at the center of the distribution $\left(\vec{z}_{1}=0\right)$. Using $\eta=\vec{z}_{1}-\vec{z}_{2}$, we expand a general Gaussian:

$$
f\left(\vec{z}_{2}\right)=\frac{1}{\Gamma} e^{-\frac{1}{2} \vec{z}_{2}^{T} \mathbb{M} \vec{z}_{2}}=\frac{1}{\Gamma} e^{-\frac{1}{2} \eta^{T} \mathbb{M} \eta} e^{-\frac{1}{2} \vec{z}_{1}^{T} \mathbb{M} \vec{z}_{2}+\vec{z}_{1} \mathbb{M} \eta}
$$

Now, keeping first order in $\vec{z}_{1}$, we use

$$
f\left(\vec{z}_{2}\right) \approx f(\eta)\left(1+\vec{z}_{1}^{T} \mathbb{M} \eta\right)
$$

The term without $\vec{z}_{1}$ integrates to 0 because of the $\hat{\Delta}$. Keeping the term proportional to $-p_{a}$, we get damping coefficients

$$
b_{a b}=\frac{4 k^{2} N}{m \Gamma} I_{a c} \mathbb{C}_{c b}
$$

where

$$
I_{a b}=\int d^{6} \eta \frac{\hat{\Delta}_{a} \hat{\Delta}_{c}}{\Delta b^{3}} e^{-\frac{1}{2} \eta_{i} \eta_{j} \mathbb{M}_{i j}} \delta(\hat{\Delta} \cdot \hat{r})
$$

For the diffusion coefficients, we can take the $0^{\text {th }}$ order term because its the leading term in this case. Setting $\vec{z}_{1}=0$, we get

$$
d_{a b}=\frac{4 k^{2} N}{m \Gamma} \int d^{6} \eta \frac{\hat{r}_{a} \hat{r}_{c}}{\Delta b^{3}} e^{-\frac{1}{2} \eta_{i} \eta_{j} \mathbb{M}_{i j}} \delta(\hat{\Delta} \cdot \hat{r})
$$

We write these for reference, but do not pursue this further. 


\section{IBS Moment Evolution}

In this section we combine together the damping and diffusion coefficients to find the second moment evolution. We will not make the Coulomb Log approximation in the following.

$$
{\frac{d \Sigma_{a b}}{d t}}^{\mathrm{IBS}}=\left\langle\frac{\left\langle\delta p_{a}\right\rangle}{\Delta t} p_{b}+\frac{1}{2} \frac{\left\langle\delta p_{a} \delta p_{b}\right\rangle}{\Delta t}\right\rangle+(a \rightarrow b)
$$

Using the expressions derived earlier for the drift and diffusion coefficients from IBS, and combining the integrals together, we get

$$
{\frac{d \Sigma_{a b}}{d t}}^{\mathrm{IBS}}=\frac{4 k^{2}}{m} \int \frac{d \vec{z}_{1} d \vec{z}_{2}}{\Delta^{2} r^{3}}\left[-\hat{\Delta}_{a} p_{1 b}+\frac{1}{2} \Delta \hat{r}_{a} \hat{r}_{b}\right] f\left(\vec{z}_{1}\right) \frac{f\left(\vec{z}_{2}\right)}{N} \delta(\hat{\Delta} \cdot \hat{r})+(a \rightarrow b)
$$

Next, consider what happens to the first term under a change of variables that interchanges 1 and 2 . The only changes will be that $p_{1} \rightarrow p_{2}$ and $\hat{\Delta} \rightarrow-\hat{\Delta}$. Thus under the integral we can make the substitution

$$
\hat{\Delta}_{a} p_{1 b} \rightarrow \frac{1}{2} \hat{\Delta}_{a}\left(p_{1 b}-p_{2 b}\right)=\frac{1}{2} \Delta \hat{\Delta}_{a} \hat{\Delta}_{b}
$$

which is the same for the term with $a \rightarrow b$ so that we can just multiply the first term by 2 . Now, factoring out the $\Delta$, and canceling one in the denominator, we have

$$
{\frac{d \Sigma_{a b}}{d t}}^{\mathrm{IBS}}=\frac{4 k^{2}}{m N} \int \frac{d \vec{z}_{1} d \vec{z}_{2}}{\Delta r^{3}}\left[\hat{r}_{a} \hat{r}_{b}-\hat{\Delta}_{a} \hat{\Delta}_{b}\right] f\left(\vec{z}_{1}\right) f\left(\vec{z}_{2}\right) \delta(\hat{\Delta} \cdot \hat{r})
$$

Next, we consider a general gaussian for $f$ :

$$
f(\vec{z})=\frac{N}{\Gamma} e^{-\frac{1}{2} z_{i} z_{j} M_{i j}}
$$

normalized so that the integral over the distribution gives $N$, the total number of particles. This means that

$$
\Gamma=(2 \pi)^{3} \sqrt{\operatorname{det} M^{-1}}
$$


To give a little more detail about the position and momentum distribution, we write

$$
M=\left(\begin{array}{cc}
\mathbb{A} & \mathbb{B} \\
\mathbb{B}^{T} & \mathbb{C}
\end{array}\right) \rightarrow f(\vec{z})=\frac{N}{\Gamma} e^{-\frac{1}{2} A_{a b} x_{a} x_{b}-B_{a b} x_{a} p_{b}-\frac{1}{2} C_{a b} p_{a} p_{b}}
$$

The combination in the integrand can then be written

$$
f\left(\vec{z}_{1}\right) f\left(\vec{z}_{2}\right)=\frac{N}{\Gamma^{2}} e^{-\frac{1}{2}\left(z_{1 i} z_{1 j}+z_{2 i} z_{2 j}\right) M_{i} j}
$$

Now, we notice that

$$
\begin{aligned}
z_{1 i} z_{1 j}+z_{2 i} z_{2 j} & =\frac{1}{2}\left(z_{1 i}+z_{2 i}\right)\left(z_{1 j}+z_{2 j}\right)+\frac{1}{2}\left(z_{1 i}-z_{2 i}\right)\left(z_{1 j}-z_{2 j}\right) \\
& =\frac{1}{2} w_{i} w_{j}+\frac{1}{2} \eta_{i} \eta_{j}
\end{aligned}
$$

with $w_{i}=z_{1 i}+z_{2 i}$ and $\eta_{i}=z_{1 i}-z_{2 i}$. We can now change variables under the integration:

$$
\int d^{6} \vec{z}_{1} d^{6} \vec{z}_{2} \ldots=\frac{1}{2^{6}} \int d^{6} \eta d^{6} w \ldots
$$

The only dependence on $w$ is in $f$. The $w$ integral is

$$
\frac{1}{\Gamma} \int d^{6} w e^{-\frac{1}{4} w_{i} w_{j} M_{i j}}=\frac{2^{3}}{\Gamma} \int d^{6} \bar{w} e^{-\frac{1}{2} \bar{w}_{i} \bar{w}_{j} M_{i j}}=2^{3}
$$

Doing the same thing to turn the $\frac{1}{4}$ in the exponent in the $\eta$ integral into a $\frac{1}{2}$, we get an additional factor of 2 . So, we are left with

$$
{\frac{d \Sigma_{a b}}{d t}}^{\mathrm{IBS}}=\frac{N k^{2}}{m \Gamma} \int \frac{d^{6} \eta}{\Delta r^{3}}\left[\hat{r}_{a} \hat{r}_{b}-\hat{d}_{a} \hat{d}_{b}\right] e^{-\frac{1}{2} \eta_{i} \eta_{j} M_{i j}} \delta(\hat{\Delta} \cdot \hat{r})
$$

To make the constants more transparent, change variables in the integral to unitless variables: $\bar{r}=\frac{r}{r_{m}}$ and $\bar{\Delta}=\frac{\Delta}{P_{0}}$. The result is that the integrand is expressed in terms of these unitless variables, and we multiply by an overall factor of $P_{0}^{2}$ :

$$
{\frac{d \bar{\Sigma}_{a b}}{d t}}^{\mathrm{IBS}}=\mathcal{A} \mathbb{K}_{a b}
$$


with

$$
\begin{gathered}
\mathcal{A}=\frac{N k^{2}}{m \Gamma}=\frac{N r_{0}^{2} c}{(2 \pi)^{3} \beta^{3} \gamma^{3} \epsilon_{x} \epsilon_{y} \sigma_{s} \sigma_{\delta}} \\
\bar{\Sigma}_{a b}=\frac{\Sigma_{a b}}{P_{0}^{2}}
\end{gathered}
$$

and

$$
\mathbb{K}_{a b}=\int \frac{d^{6} \eta}{\Delta r^{3}}\left[\hat{r}_{a} \hat{r}_{b}-\hat{d}_{a} \hat{d}_{b}\right] e^{-\frac{1}{2} \eta_{i} \eta_{j} \overline{\mathbb{M}}_{i j}} \delta(\hat{\Delta} \cdot \hat{r})
$$

Now the matrix $\overline{\mathrm{M}}$ is unitless, positions measured in units of $r_{m}$, momenta in terms of $P_{0} . \Gamma$ has units of length cubed times momentum cubed. So overall

$$
\left[\frac{P_{0}^{2} k^{2}}{m \Gamma}\right]=\left[\frac{p^{2} l^{2} p^{4}}{m l^{3} p^{3}}\right]=\left[\frac{p^{3}}{m l}\right]=\left[\frac{p^{2}}{t}\right]
$$

as we expect. The dependence on $r_{m}$ is interesting. From the above expression, it looks at first glance as though we could have chosen any value for $r_{m}$ and the integrand would have same form. The hidden point is that the integrand diverges at small $r$ and whereas previously we cut the integrand off at $r=r_{m}$, now we cut it off at $\bar{r}=1$. So the choice of $r_{m}$ as a unit really is special. Next, we consider spherical coordinates for both $\vec{\Delta}$ and $\vec{r}$. The delta function is going to do one of the angular integrals for us. We have a choice of which one. Define the following vectors:

$$
\hat{e}_{1}=\left(\begin{array}{c}
\sin \theta \cos \phi \\
\sin \theta \sin \phi \\
\cos \theta
\end{array}\right), \quad \hat{e}_{2}=\left(\begin{array}{c}
\cos \theta \cos \phi \\
\cos \theta \sin \phi \\
-\sin \theta
\end{array}\right), \quad \hat{e}_{3}=\left(\begin{array}{c}
-\sin \phi \\
\cos \phi \\
0
\end{array}\right)
$$

Then we can either let $\hat{\Delta}=\hat{e}_{1}$ and $\hat{b}=\cos \alpha \hat{e}_{2}+\sin \alpha \hat{e}_{3}$ or $\hat{b}=\hat{e}_{1}$ and $\hat{\Delta}=$ $\cos \alpha \hat{e}_{2}+\sin \alpha \hat{e}_{3}$.

$$
\mathbb{K}_{a b}=\int d \Delta d r d \Omega \frac{\Delta}{r} h_{a b} e^{-\frac{1}{2}\left(h_{1} r^{2}+h_{2} r \Delta+h_{3} \Delta^{2}\right)} \delta(\hat{\Delta} \cdot \hat{r})
$$

where

$$
h_{a b}=\left(\hat{r}_{a} \hat{r}_{b}-\hat{\Delta}_{a} \hat{\Delta}_{b}\right)
$$


and

$$
\begin{aligned}
h_{1} & =\overline{\mathbb{A}}_{a b} \hat{b}_{a} \hat{b}_{b} \\
h_{2} & =\overline{\mathbb{B}}_{a b} \hat{b}_{a} \hat{\Delta}_{b}+\overline{\mathbb{B}}_{a b} \hat{b}_{b} \hat{\Delta}_{a} \\
h_{3} & =\overline{\mathbb{C}}_{a b} \hat{\Delta}_{a} \hat{\Delta}_{b}
\end{aligned}
$$

Now, we do the $r$ and $\Delta$ integrals. We can see that the integral will diverge at $r=0$, and hence we need a minimum distance cut-off $r_{m}$. We would like to consider a $\Delta$ dependent cut-off. As an approximation, for now, we will take $r_{m}$ to be a constant. Then, we can do the $\Delta$ integration first:

$$
\mathbb{K}_{a b}=\int d \Omega d \alpha h_{a b} \int d r \frac{e^{-\frac{h_{1}}{2} r^{2}}}{r} I_{\Delta}(r, \Omega)
$$

and

$$
I_{\Delta}(r, \Omega)=\int_{0}^{\infty} \Delta e^{-\frac{h_{3}}{2} \Delta^{2}-\frac{a}{2} \Delta} d \Delta
$$

with $a=h_{2} r$. We now do the following to isolate the $r$ dependent part from the non- $r$ dependent part.

$$
\begin{aligned}
I_{\Delta} & =\frac{1}{h_{3}} \int\left(h_{3} \Delta-\frac{a}{2}+\frac{a}{2}\right) e^{-\frac{h_{3}}{2} \Delta^{2}+\frac{a}{2} \Delta} d \Delta \\
& =\left.\frac{1}{h_{3}} e^{-\frac{h_{3}}{2} \Delta^{2}+a \Delta}\right|_{0} ^{\infty}+\frac{a}{h_{3}} \int_{0}^{\infty} e^{-\frac{h_{3}}{2} \Delta^{2}+a \Delta} \\
& =\frac{1}{h_{3}}+\frac{a}{h_{3}} e^{\frac{-a^{2}}{h_{3}}} \int_{0}^{\infty} e^{-\frac{h_{3}}{2}\left(\Delta-\frac{a}{h_{3}}\right)^{2}} d \Delta \\
& =\frac{1}{h_{3}}+\frac{a}{h_{3}} e^{\frac{-a^{2}}{h_{3}}}\left\{\frac{\sqrt{\pi}}{\sqrt{2 h_{3}}}\left(-1+\operatorname{Erf}\left(\frac{a}{2 \sqrt{h_{3}}}\right)\right)\right\}
\end{aligned}
$$

The 2nd term is thus:

$$
\begin{aligned}
& \frac{h_{2}}{h_{3}} \int e^{-\frac{h_{1}}{2} r^{2}-h_{2} \Delta r-\frac{h_{3}}{2} \Delta^{2}} d r \\
= & \frac{q}{2 h_{3}}\left(\pi-2 \tan ^{-1}(q)\right)
\end{aligned}
$$


where

$$
q=\frac{h_{2}}{\sqrt{h_{1} h_{3}-h_{2}^{2}}}
$$

The fact that $h_{1} h_{3}-h_{2}^{2}>0$ follows from the fact that $\mathbb{M}_{i j}$ is positive definite, i.e. $\mathbb{M}_{i j} z_{i} z_{j}>0$ for all $z_{i}$. Now, we do the $r$ integral by using the fact that

$$
\int_{1}^{\infty} \frac{e^{-\frac{\bar{h}_{1}}{2} r^{2}}}{r} d r=-\log \left(\frac{h_{1}}{2}\right)-\gamma_{E}+O\left(\bar{h}_{1}\right)
$$

which can be derived by changing variables to $x=r^{2}$, integrating by parts and then using the identity

$$
\int_{0}^{\infty} \log (x) e^{-C x} d x=-\frac{1}{C}\left(\log C+\gamma_{E}\right)
$$

with $\gamma_{E}$ the Euler Gamma constant equal to $0.577 \ldots$ The order $\bar{h}_{1}$ remainder comes from the integral from 0 to 1 . Since $\bar{h}_{1}<\frac{r_{m}^{2}}{\min \left(\sigma_{x}^{2}, \sigma_{y}^{2}, \sigma_{s}{ }^{2}\right)}$ we can ignore this term in the typical situation. So we have

$$
\mathbb{K}_{a b}^{\mathrm{IBS}}=\int d \Omega \frac{-h_{a b}}{\bar{h}_{3}}\left[\left(\log \left(\bar{h}_{1}\right)+\gamma_{E}-\log (2)\right)+\frac{q}{2}\left(\pi-2 \tan ^{-1}(q)\right)\right]
$$

The third term here $\frac{q h_{a b}}{h_{3}}$ integrates to 0 from the $\alpha$ integral. So we have

$$
\mathbb{K}_{a b}^{\mathrm{IBS}}=\int d \Omega\left[\frac{-h_{a b}}{\bar{h}_{3}}\left(\log \left(\bar{h}_{1}\right)+\gamma_{E}\right)-q \tan ^{-1}(q)\right]
$$

Let's ignore the 2 nd term for now, and do the $\alpha$ integral in the 1 st term. We'd like to integrate

$$
\int_{0}^{2 \pi} \frac{h_{a b}}{h_{3}} d \alpha
$$

This is the relevant $\alpha$ integral only if we've chosen the coordinates so that $h_{1}$ doesn't depend on $\alpha$. i.e $\hat{r}=\hat{e}_{1}$ and $\hat{\Delta}=\cos \alpha \hat{e}_{2}+\sin \alpha \hat{e}_{3}$.

We can then write $h_{3}$ in the form

$$
\begin{aligned}
h_{3}=a \cos ^{2} \alpha+b \sin \alpha \cos \alpha+c \sin ^{2} \alpha & =\frac{1}{2}\left(a_{+}+a_{-} \cos 2 \alpha+b \sin 2 \alpha\right) \\
& =a_{+}+\sqrt{a_{-}^{2}+b^{2}} \cos (2 \alpha+\psi)
\end{aligned}
$$


where

$$
\sin \psi=\frac{-b}{\sqrt{a_{-}^{2}+b^{2}}}
$$

with $a_{+}=a+c$ and $a_{-}=a-c$, and

$$
\begin{aligned}
a & =\hat{e}_{2 a} \hat{e}_{2 b} \overline{\mathbb{C}}_{a b} \\
b & =2 \hat{e}_{2 a} \hat{e}_{3 b} \overline{\mathbb{C}}_{a b} \\
c & =\hat{e}_{3 a} \hat{e}_{3 b} \overline{\mathbb{C}}_{a b}
\end{aligned}
$$

Now, $\hat{r}_{a} \hat{r}_{b}$ doesn't depend on $\alpha$, but $\hat{\Delta}_{a} \hat{\Delta}_{b}$ does. So for $h_{a b}$ we can write

$$
\begin{aligned}
h_{a b} & =\hat{r}_{a} \hat{r}_{b}-u_{a b} \cos ^{2} \alpha-v_{a b} \sin \alpha \cos \alpha-w \sin ^{2} \alpha \\
& =\hat{r}_{a} \hat{r}_{b}-\frac{1}{2}\left(u_{a b}^{+}+u_{a b}^{-} \cos 2 \alpha+w \sin 2 \alpha\right)
\end{aligned}
$$

with $u_{a b}^{+}=u_{a b}+w_{a b}$ and $u_{a b}^{-}=u_{a b}-w_{a b}$. For $u_{a b}, v_{a b}, w_{a b}$ we have explicitly

$$
\begin{aligned}
u_{a b} & =\hat{e}_{2 a} \hat{e}_{2 b} \\
v_{a b} & =\hat{e}_{2 a} \hat{e}_{3 b}+\hat{e}_{2 b} \hat{e}_{3 a} \\
w_{a b} & =\hat{e}_{3 a} \hat{e}_{3 b}
\end{aligned}
$$

Our $\alpha$ integral now becomes

$$
\begin{aligned}
\int_{0}^{2 \pi} \frac{h_{a b}}{\bar{h}_{3}} d \alpha & =\left(\hat{r}_{a} \hat{r}_{b}-\frac{1}{2} u_{a b}^{+}\right) \int_{0}^{2 \pi} \frac{d \alpha}{a_{+}+\sqrt{a_{-}^{2}+b^{2}} \cos (2 \alpha+\psi)} \\
& -\frac{1}{2} u_{a b}^{-} \int_{0}^{2 \pi} \frac{\cos 2 \alpha d \alpha}{a_{+}+a_{-} \cos 2 \alpha+b \sin 2 \alpha} \\
& -\frac{1}{2} v_{a b} \int_{0}^{2 \pi} \frac{\sin 2 \alpha d \alpha}{a_{+}+a_{-} \cos 2 \alpha+b \sin 2 \alpha}
\end{aligned}
$$

The first integral can be done by changing variables- first shifting by $\psi$ then scaling by 2 . Doing this does not introduce any overall factors. Then we use the formula

$$
\int_{0}^{2 \pi} \frac{d \alpha}{A+B \cos \alpha}=\frac{2 \pi}{\sqrt{A^{2}-B^{2}}}
$$


so that the first term becomes

$$
\left(\hat{r}_{a} \hat{r}_{b}-\frac{1}{2} u_{+}\right) \frac{2 \pi}{\sqrt{4 a c-b^{2}}}
$$

where we've used the fact that $a_{+}^{2}-a_{-}^{2}=4 a c$. For the second and third terms, we use the formulas

$$
\int \frac{\cos \alpha}{A+B \cos \alpha+C \sin \alpha}=\frac{2 \pi B}{B^{2}+C^{2}-A^{2}-A \sqrt{A^{2}-B^{2}-C^{2}}}
$$

and

$$
\int \frac{\sin \alpha}{A+B \cos \alpha+C \sin \alpha}=\frac{2 \pi C}{B^{2}+C^{2}-A^{2}-A \sqrt{A^{2}-B^{2}-C^{2}}}
$$

Now, we compute

$$
A^{2}-B^{2}-C^{2}=4 a c-b^{2} \equiv q
$$

and we get

$$
\int_{0}^{2 \pi} \frac{h_{a b}}{h_{3}} d \alpha=2 \pi\left(\frac{\left(\hat{r}_{a} \hat{r}_{b}-\frac{1}{2} u_{a b}^{+}\right)}{\sqrt{q}}+\frac{1}{2} \frac{\left(\left(u_{a b}-w_{a b}\right)(a-c)+v_{a b} b\right)}{q+(a+c) \sqrt{q}}\right)=\Theta_{a b}(\theta, \phi)
$$

Our final answer thus becomes

$$
\mathbb{K}_{a b}^{\mathrm{IBS}}=\int d \cos \theta d \phi\left[-\Theta_{a b}(\theta, \phi)\left(\log \left(h_{1}\right)+\gamma_{E}\right)\right]+\int d \alpha d \cos \theta d \phi q \frac{h}{h_{3}} \tan ^{-1}(q)
$$

\section{Connection to Bjorken-Mtingwa and Coulomb Logarithm}

Throwing away the terms in the growth rate without logarithmic divergence, and ignoring the $\gamma_{E}-\ln 2$ we have:

$$
\frac{1}{\tau^{(a)}}=\frac{1}{2} \mathcal{A} C_{a b}^{(a)} \mathbb{K}_{a b}=\frac{1}{2} \mathcal{A} C_{a b}^{(a)} \int d \Omega \frac{h_{a b}}{h_{3}}\left(\log \left(h_{1}\right)\right)
$$


with

$$
\mathcal{A}=\frac{N r_{0}^{2} c}{32 \pi^{3} \beta^{3} \gamma^{4} \epsilon_{x} \epsilon_{y} \sigma_{s} \sigma_{\delta}}
$$

If we approximate the term in the parentheses as a constant, and call it $2 L_{c}$ (the 2 is because the argument in the logarithm is squared), we get

$$
\begin{gathered}
\mathbb{K}_{a b}=2 L_{c} \mathbb{K}_{a b}^{\mathrm{BM}} \\
\mathbb{K}^{\mathrm{BM}}=\int d \Omega \frac{h_{a b}}{h_{3}}=\int d \alpha d \cos \theta d \phi \frac{\left(\hat{r}_{a} \hat{r}_{b}-\hat{\Delta}_{a} \hat{\Delta}_{b}\right)}{\overline{\mathbb{C}}_{a b} \hat{\Delta}_{a} \hat{\Delta}_{b}}
\end{gathered}
$$

Now, if we make the choice

$$
\hat{r}=\hat{b}_{1} \cos \alpha+\hat{b}_{2} \sin \alpha
$$

and do the $\alpha$ integral we get

$$
\mathbb{K}_{a b}^{\mathrm{BM}}=\pi \int d \cos \theta d \phi \frac{\delta_{a b}-3 \hat{\Delta}_{a} \hat{\Delta}_{b}}{\overline{\mathbb{C}}_{a b} \hat{\Delta}_{a} \hat{\Delta}_{b}}
$$

Now using the relation

$$
2 \int_{0}^{\infty} \Delta e^{-y \Delta^{2}} d \Delta=\frac{1}{y}
$$

we get that

$$
\begin{aligned}
\mathbb{K}_{a b}^{\mathrm{BM}} & =2 \pi \int d \Delta d \omega \Delta\left(\delta_{a b}-3 \hat{\Delta}_{a} \hat{\Delta}_{b}\right) e^{-\Delta^{2} C_{a b} \hat{\Delta}_{a} \hat{\Delta}_{b}} \\
& =2 \pi \int \frac{d \vec{\Delta}}{\Delta}\left(\delta_{a b}-3 \hat{\Delta}_{a} \hat{\Delta}_{b}\right) e^{-\vec{\Delta}_{a} \vec{\Delta}_{b} C_{a b}}
\end{aligned}
$$

Now, following B-M, using the identity

$$
\frac{1}{\Delta^{3}}=\int_{0}^{\infty} \frac{d \lambda \sqrt{\lambda}}{4 \sqrt{\pi}} e^{\frac{-\lambda \Delta^{2}}{4}}
$$

we get

$$
\mathbb{K}^{\mathrm{BM}}=2 \pi^{2} \int_{0}^{\infty} d \lambda \frac{\sqrt{\lambda}}{\operatorname{det}(\Lambda)}\left(\delta_{a b} \operatorname{Tr}\left(\Lambda^{-1}\right)-3 \Lambda_{a b}^{-1}\right)
$$


where $\Lambda=\overline{\mathbb{C}}-\lambda I$. So, for the growth rate we get

$$
\frac{1}{\tau^{(a)}}=\frac{1}{2} C_{a b}^{(a)} 2 L_{c}\left(2 \pi^{2}\right) \frac{N r_{0}^{2} c}{32 \pi^{3} \beta^{3} \gamma^{4} \epsilon_{x} \epsilon_{y} \sigma_{s} \sigma_{\delta}} \int_{0}^{\infty} d \lambda \frac{\sqrt{\lambda}}{\operatorname{det}(\Lambda)}\left(\delta_{a b} \operatorname{Tr}\left(\Lambda^{-1}\right)-3 \Lambda_{a b}^{-1}\right)
$$

\section{Lebedev Approximation}

Lebedev et. al. [51] considers the limit where $\sigma_{p_{z}} \ll \sigma_{p_{x, y}}$ Here, this means $\mathbb{C}_{33} \gg$ $\mathbb{C}_{11,22}$. We approximate the integrals

$$
\mathbb{K}_{a b}^{\mathrm{BM}}=\int d \cos \theta d \phi \frac{\delta_{a b}-3 \hat{\Delta}_{a} \hat{\Delta}_{b}}{\mathbb{C}_{11}(\sin \theta \cos \phi)^{2}+\mathbb{C}_{22}(\sin \theta \sin \phi)^{2}+\mathbb{C}_{33}(\cos \theta)^{2}}
$$

Since usually $\mathbb{K}_{33}$ is most important let's consider that. The integral we need can be written

$$
\int_{0}^{1} d x \frac{1-3 x^{2}}{C_{1}\left(1-x^{2}\right)+\mathbb{C}_{33} x^{2}}
$$

where $C_{1}=\mathbb{C}_{11} \sin ^{2} \phi+\mathbb{C}_{22} \cos ^{2} \phi$ and $C 1 \ll C_{33} \forall \phi$. Now, define $\epsilon=C_{1} / \mathbb{C}_{33}$ and we have

$$
\frac{1}{\mathbb{C}_{33}} \int_{0}^{1} d x \frac{1-3 x^{2}}{x^{2}+\epsilon\left(1-x^{2}\right)}=\frac{3}{\epsilon-1}-\frac{(1+2 \epsilon) \tan ^{-1}\left(\sqrt{\frac{1}{\epsilon}-1}\right)}{(\epsilon-1) \sqrt{1-\epsilon} \sqrt{\epsilon}}
$$

The first term is $\approx 3$ and the second term is $\approx 1 / \sqrt{\epsilon}$, so for large $\epsilon$ we can ignore the first term and just keep the $1 / \sqrt{\epsilon}$ term in the second. Using the fact that $\tan ^{-1}$ of a large number approaches $\pi / 2$, we approximate the integral as $\frac{\pi}{2 \sqrt{\epsilon}}$ so that the integral becomes

$$
\mathbb{K}_{a b}^{\mathrm{BM}} \approx \frac{\pi}{\sqrt{\mathbb{C}_{33}}} \int_{0}^{2 \pi} d \phi \frac{1}{\sqrt{\mathbb{C}_{11} \sin ^{2} \phi+\mathbb{C}_{22} \cos ^{2} \phi}}
$$

We have put a factor of 2 back in since the integral was really from $x=-1$ to $x=1$ and we used symmetry to make it from 0 to 1 . Note that when we put the prefactor back in for the growth rate, the dependence on $\sigma_{\delta}$ drops out, as Lebedev has noted. 


\section{Bibliography}

[1] C. Steier et al., "Intra-beam scattering and minimum achievable emittance in the Advanced Light Source," SLAC-PUB-9364 Contributed to IEEE Particle Accelerator Conference (PAC 2001), Chicago, Illinois, 18-22 Jun 2001

[2] S. Chandrasekar, Rev. Mod. Phys. 15, 1 (1943)

[3] E. P. Lee, The Astrophysical Journal, 151, (1968)

[4] H. Kandrup, Phys. Rep., Volume 63, 1, p. 1-59. (1980)

[5] M. Venturini, "Intrabeam scattering and wake field effects in low emittance electron rings," SLAC-PUB-9355 Presented at IEEE Particle Accelerator Conference (PAC2001), Chicago, Illinois, 18-22 Jun 2001

[6] J. Qiang, R. D. Ryne, and S. Habib, "Self-Consistent Langevin Simulation of Coulomb Collisions in Charged-Particle Beams," Technical Paper, SC2000 (Dallas, November 2000)

[7] J. Wei, A. Fedotov, W. Fischer, N. Malitsky, G. Parzen, and J. Qiang, AIP Conference Proceedings 773, Issue 1, pp. 389-393 (2005)

[8] G. Parzen, "Intrabeam scattering growth rates for a bi-gaussian distribution," arXiv:physics/0410028.

[9] V. Lebedev and S. Nagaitsev, FERMILAB-CONF-02-099-T Presented at 8th European Particle Accelerator Conference (EPAC 2002), Paris, France, 3-7 Jun 2002 
[10] Rosenbluth M.N., MacDonald W. and Judd D., Phys. Rev. 103,507 (1956)

[11] L.D. Landau and E.M. Lifshitz, Statistical Physics, Addison-Wesley, Reading, MA (1958)

[12] A. Piwinski, Tech. Rep. HEAC 74, Stanford, (1974); A. Piwinski, Sec.2.3.4, Handbook of Accelerator Physics and Engineering, ed. A. Chao and M. Tigner, World Scientific (2002).

[13] S. K. Mtingwa and J. D. Bjorken, Part. Accel. 13, 115 (1983).

[14] E. W. Kolb and M. S. Turner, The Early Universe (Addison-Wesley, Reading, MA, 1990), p. 342.

[15] R. Cohen, L. Spitzer and P. M. Routly, Phys. Rev. 80, 230238 (1950)

[16] W. B. Thompson and J. Hubbard, Rev. Mod. Phys. 32, 714718 (1960)

[17] R. Farouki and E. Salpeter, The Astrophysical Journal, 427, 676-683 (1994)

[18] B. Nash, J. Wu, K. Bane and A. Chao, "A new analysis of intrabeam scattering," SLAC-PUB-10736, Proc. Part. Accel. Conf., Portland, 2003.

[19] A. Piwinski, DESY-90-113

[20] V. Lebedev, Private Communication (2006)

[21] K. Kubo and K. Oide, Phys. Rev. ST Accel. Beams 4, 124401 (2001).

[22] E.D. Courant and H.S. Snyder, Annals of Phys. 3, 1 (1958)

[23] D.A. Edwards and L.C. Teng, IEEE Trans. Nucl. Sci. NS-20, 885 (1973)

[24] A. Wolski Phys. Rev. ST Accel. Beams 9, 024001 (2006)

[25] R. Calaga, R. Tomas and A. Franchi, Phys. Rev. ST Accel. Beams 8, 034001 (2005).

[26] Y. T. Yan and Y. Cai, SLAC-PUB-10371 
[27] Y. Cai, Phys. Rev. E 68, 036501 (2003).

[28] D. Sagan and D. Rubin, Phys. Rev. ST Accel. Beams 2, 074001 (1999)

[29] B. Nash, J. Wu, A. Chao, Phys. Rev. ST Accel. Beams 9, 032801 (2006)

[30] A. Schoch, CERN-57-21

[31] G. Guignard, CERN-76-06

[32] K. Ohmi, K. Hirata, and K. Oide, Phys. Rev. E 49, 751 (1994)

[33] F. Ruggiero, E. Picasso, L. Radicati, Annals of Phys. 197, 439 (1990)

[34] A. W. Chao and M. J. Lee, J. Appl. Phys. 47, 4453 (1976).

[35] G. H. Hoffstaetter and A. W. Chao, Phys. Rev. ST Accel. Beams 7, 071002 (2004) [arXiv:physics/0405107].

[36] M. Sands, SLAC Report No. SLAC-121 (1970) (unpublished)

[37] A. Chao, J. Appl. Phys. 50, 595 (1979)

[38] E. Forest, "Beam dynamics: A new attitude and framework," Amsterdam, Netherlands: Harwood (1998)

[39] H. Risken, "The Fokker-Planck Equation", Springer-Verlag, New York (1989)

[40] A. W. Chao, SSC-105 Lecture given at Joint US/CERN Accelerator School, Topical Course on Frontiers of Particle Beams, South Padre Island, Oct 1986

[41] K. Huang, Statistical Mechanics, Wiley (1963)

[42] H. E. Kandrup, I. V. Sideris and C. L. Bohn, Phys. Rev. ST Accel. Beams 7, 014202 (2004) [arXiv:physics/0303022].

[43] See for example, A. Piwinski, Ref. [44], pg. 72

[44] A.W.Chao and M.Tigner, ed. "Handbook of accelerator physics and engineering," (1999) 
[45] S. Nagaitsev, Phys. Rev. ST Accel. Beams 8, 064403 (2005).

[46] K. Kubo, S. K. Mtingwa and A. Wolski, Phys. Rev. ST Accel. Beams 8, 081001 (2005).

[47] A. Burov, AIP Conf. Proc. 773, 404 (2005).

[48] K. L. F. Bane, "An accurate, simplified model of intrabeam scattering," arXiv:physics/0205058.

[49] G. Parzen, Nucl. Instrum. Meth. A 256, 231 (1987).

[50] V. Lebedev, "Single and multiple intrabeam scattering in hadron colliders," AIP Conf. Proc. 773, 440 (2005).

[51] Lebedev, V.A.; Labrador, A.; Madsen, N.; Hangst, J.S.; Nucl. Instrum. Methods in Physics Research A. 391, 176, (1997)

[52] G. Parzen, arXiv:physics/0405019.

[53] M. Conte and M. Martini, Part. Accel. 17, 1 (1985).

[54] A. G. Ruggiero, FERMILAB-FN-0413 Submitted to Proc. of Conf. on Intersections between Particle and Nuclear Physics, Steamboat Springs, CO, May 23-30, 1984

[55] T. Toyamasu, Part. Accel. 40, 161 (1993).

[56] K. Takayama, FERMILAB-FN-0336

[57] W. Kells, FERMILAB-TM-1093

[58] PEP-II: An Asymmetric B Factory, Conceptual Design Report, SLAC-418, June (1993)

[59] J. Seeman et al., SLAC-PUB-11727 Prepared for Particle Accelerator Conference (PAC 05), Knoxville, Tennessee, 16-20 May 2005 
[60] KEKB B-Factory Design Report, KEK-Report 95-7, (1995)

[61] J. Wei, Prepared for 1993 IEEE Particle Accelerator Conference (PAC 93), Washington, DC, 17-20 May 1993

[62] T. O. Raubenheimer, Part. Accel. 45, 111 (1994).

[63] R. H. Helm, M. J. Lee, P.L. Morton, M. Sands, IEEE Trans. Nucl. Sci. NS-20, $900(1973)$

[64] J. Wu, B. Nash, A.W. Chao, "Equilibrium Beam Invariants of an Electron Storage Ring with Linear x-y Coupling", SLAC-PUB-11277, Proc. Part. Accel. Conf., Knoxville, 2005.

[65] K.W. Robinson, Phys. Rev. 111 373, (1958)

[66] D.P. Barber, K. Heinemann, H. Mais and G. Ripken, DESY-91-146 (1991)

[67] David J. Griffiths, "Introduction to Quantum Mechanics", Prentice Hall, New Jersey (1994)

[68] R. Palmer, Snowmass DPF Summer Study 1988, p. 613 (1988)

[69] J. Struckmeier, Phys. Rev. ST Accel. Beams 3, 034202 (2000).

[70] J. Struckmeier, Prepared for JAS'2000: Accelerator School on Frontiers of Accelerator Technology: High Quality Beams (a river-boat between St. Petersburg and Moscow), St. Petersburg - Moscow, Russia, 1-7 Jul 2000

[71] A. Kabel, SLAC-PUB-8841 Contributed to IEEE Particle Accelerator Conference (PAC 2001), Chicago, Illinois, 18-22 Jun 2001

[72] W. Fischer, R. Connolly, S. Tepikian, J. van Zeijts and K. Zeno, Prepared for 8th European Particle Accelerator Conference (EPAC 2002), Paris, France, 3-7 Jun 2002

[73] W. Fischer, private communication, 2005

[74] A. W. Chao, AIP Conf. Proc. 127, 201 (1985). 